JOURNAL OF THE

AMERICAN MATHEMATICAL SOCIETY

Volume 23, Number 4, October 2010, Pages 1041-1118

S 0894-0347(2010)00671-6

Article electronically published on May 24, 2010

\title{
NONLINEAR WAVE PROPAGATIONS OVER A BOLTZMANN SHOCK PROFILE
}

\author{
SHIH-HSIEN YU
}

\section{Contents}

1. Introduction 1042

2. Preliminaries 1051

2.1. Basic theories on hyperbolic conservation laws

1051

2.2. Basic theories for the Boltzmann equation on the linear and nonlinear collision operators

2.3. Macro-micro decompositions around a hydrodynamic macroscopic state $\overrightarrow{\boldsymbol{v}}$

2.4. Boltzmann shock layers

2.5. The Green's function of a linearized Boltzmann equation around a global Maxwellian

3. Comparisons on the Green's functions around the end states of a shock wave 1057

4. Split wave propagation over a Boltzmann shock profile: $\mathbb{T}-\mathbb{C}$ scheme

4.1. A basic transverse initial value problem and outer approximation

4.2. The $\mathbb{T}-\mathbb{C}$ scheme: Transverse wave operators, compressive wave operators, and local wave front tracing

4.3. Mild transverse initial data and the $\mathbb{T}-\mathbb{C}$ schemes

5. Estimates on operators

5.1. Estimates on the transverse wave operator

5.2. Estimates on local wave front tracing

5.3. Energy estimates

5.4. Energy-pointwise estimates 1088

6. Convergence of the $\mathbb{T}-\mathbb{C}$ scheme

and global time estimate around shock fronts $\quad 1092$

6.1. Convergence of the $\mathbb{T}-\mathbb{C}$ scheme for the basic initial data 1092

6.2. A representation of solutions with center $y_{0}$ away from zero 1101

6.3. Summary on estimates of the linearized equation around the shock layer

Received by the editors February 24, 2009 and, in revised form, March 26, 2010. 2010 Mathematics Subject Classification. Primary 35L65, 35L67, 35Q20, 35E05.

This paper is supported by start-up grant R-146-000-108-133 of the National University of Singapore. The author thanks Professor Tai-Ping Liu for introducing him to the project on Boltzmann shock waves. 
7. Nonlinear stability of a shock layer (Proof of Main Theorem) 1105

Appendix A. Computational lemmas and estimates 1110

References

\section{INTRODUCTION}

In this paper we are interested in the wave behavior of planar wave perturbations around a Boltzmann shock profile. Consider the Boltzmann equation for the hard sphere models

$$
\partial_{t} F(x, t, \xi)+\xi^{1} \partial_{x} F(x, t, \xi)=\frac{1}{\kappa} Q(F)(x, t, \xi), x \in \mathbb{R}, t>0, \xi \equiv\left(\xi^{1}, \xi^{2}, \xi^{3}\right) \in \mathbb{R}^{3},
$$

where the nonlinear collision operator $Q$ is

$$
\begin{array}{r}
Q(H)(\xi) \equiv \mathrm{B}(H, H)(\xi), \\
\mathrm{B}(H, K) \equiv \int_{\substack{\left(\xi_{*}, \Omega\right) \in \mathbb{R}^{3} \times S^{2} \\
\left(\xi-\xi_{*}\right) \cdot \Omega>0}} \frac{\left(-H(\xi) K\left(\xi_{*}\right)-K(\xi) H\left(\xi_{*}\right)+H\left(\xi^{\prime}\right) K\left(\xi_{*}^{\prime}\right)+K\left(\xi^{\prime}\right) H\left(\xi_{*}^{\prime}\right)\right)}{2} \\
\cdot\left|\left(\xi-\xi_{*}\right) \cdot \Omega\right| d \xi_{*} d \Omega,
\end{array}
$$

where

$$
\left\{\begin{array}{l}
\xi^{\prime}=\xi-\left[\left(\xi-\xi_{*}\right) \cdot \Omega\right] \Omega, \\
\xi_{*}^{\prime}=\xi_{*}+\left[\left(\xi-\xi_{*}\right) \cdot \Omega\right] \Omega
\end{array}\right.
$$

and where $\kappa>0$ is the Knudsen number. The Boltzmann shock profiles are traveling waves $F(x, t, \xi)=\varphi(x-s t, \xi)$. By Galilean transformation, we can assume for simplicity that the speed $s=0$ and the shock profile satisfies

$$
\left\{\begin{array}{l}
\xi^{1} \partial_{x} \varphi=Q(\varphi) \\
\lim _{x \rightarrow \pm \infty} \varphi(x)=\mathrm{M}_{ \pm}, \quad \mathrm{M}_{ \pm} \equiv \mathrm{M}_{\left[\rho_{ \pm}, u_{ \pm}, \boldsymbol{T}_{ \pm}\right]} .
\end{array}\right.
$$

Here the end states $\mathbf{M}_{ \pm}$are Maxwellian states with end states $\left(\rho_{ \pm}, u_{ \pm}, \boldsymbol{T}_{ \pm}\right)$of a stationary shock wave:

$$
\mathrm{M}_{[\varrho, v, \theta]} \equiv \frac{\varrho}{(2 \pi \theta)^{3 / 2}} \exp \left[-\frac{\left(\xi^{1}-v\right)^{2}+\left|\xi^{2}\right|^{2}+\left|\xi^{3}\right|^{2}}{2 \theta}\right], \varrho, \theta>0 .
$$

The Boltzmann equation is a kinetic equation to model gases in a thermal nonequilibrium state. The simplest related macroscopic equations are the Euler equations in gas dynamics:

$$
\left\{\begin{array}{l}
\rho_{t}+(\rho u)_{x}=0 \\
(\rho u)_{t}+\left(\rho u^{2}+\rho \boldsymbol{T}\right)_{x}=0, \\
\frac{1}{2}\left(\rho u^{2}+3 \rho \boldsymbol{T}\right)_{t}+\frac{1}{2}\left[u\left(\rho u^{2}+5 \rho \boldsymbol{T}\right)\right]_{x}=0 .
\end{array}\right.
$$

Euler equations can be obtained from the Boltzmann equation by assuming that the gas is in thermal equilibrium, that is, the distribution functions are local Maxwellians. The derivation for the Navier-Stokes equations in gas dynamics from the Boltzmann equation is based on the assumption that the gas is approaching thermal equilibrium. A Boltzmann shock profile is a permanent thermal nonequilibrium profile connecting two thermo-equilibrium states. Though the fluid dynamics equations do not model the profiles, the end states of a Boltzmann shock 
profile are governed by the Rankine-Hugoniot condition for the fluid dynamics equations:

$$
\left\{\begin{array}{l}
u_{+} \rho_{+}-u_{-} \rho_{-}=0, \\
\rho_{+}\left(\left|u_{+}\right|^{2}+\boldsymbol{T}_{+}\right)-\rho_{-}\left(\left|u_{-}\right|^{2}+\boldsymbol{T}_{-}\right)=0, \\
u_{+} \rho_{+}\left(\left|u_{+}\right|^{2}+5 \boldsymbol{T}_{+}\right)-u_{-} \rho_{-}\left(\left|u_{-}\right|^{2}+5 \boldsymbol{T}_{-}\right)=0 .
\end{array}\right.
$$

The main interest in this paper is to give pointwise estimates on the planar wave perturbations $F(x, t, \xi)-\varphi(x)$ for all $(x, t) \in \mathbb{R} \times \mathbb{R}_{+}$. We will make use of the basic macroscopic information from the fluid dynamics equations. For instance, for forward shocks (i.e. 3 -shock waves), there is the basic compressibility condition of the form

$$
\left\{\begin{array}{l}
u_{+}+\sqrt{5 \boldsymbol{T}_{+} / 3}<0<u_{-}+\sqrt{5 \boldsymbol{T}_{-} / 3} \\
u_{-}<0
\end{array}\right.
$$

In the asymptotic analysis based on the Hilbert expansion for the hydrodynamics limits problems by Grad [5], Sone [22, three singular slips, the initial layer, boundary layer, and shock layer, were excluded from the hydrodynamic regimes. Grad [5] proposed to study the time asymptotic behaviors of these slips on the level of the original kinetic equation for the purpose of developing a general asymptotic expansion theory with singular nonhydrodynamic structures. With the structure of the boundary layers, the purely kinetic phenomenon such as the thermal creep flows, the negative temperature gradient flows, ghost effects, etc., had been established for steady flows by Sone, Aoki, et al., [22]. Shock waves are the interior nonlinear waves. For the compressible Navier-Stokes equations there are also the interior nonlinear waves of contact layers and rarefaction waves. The microscopic parts in the contact layers and rarefaction waves of the Boltzmann equation will converge to zero. Thus the Navier-Stokes equation and the Boltzmann equation are time asymptotically equivalent on the level of contact layers and rarefaction waves. As a consequence, the time-asymptotic analysis for these two waves on the level of the Navier-Stokes equations can be generalized to the Boltzmann equations. In contrast to this, the microscopic part of the Boltzmann shock profile is time-invariant and the analysis designed for the Navier-Stokes equation can be served as a first step but is not sufficient for the Boltzmann equation. The time-asymptotic stability of the shock profile for a general planar wave perturbation has not been satisfactorily resolved for the compressible Navier-Stokes equation. This and Grad's proposal motivate the present development of a general scheme to build the Green's function for the linearized Boltzmann equation around the Boltzmann shock profile to analyze the time-asymptotic stability problem.

By letting $\overrightarrow{\boldsymbol{u}} \equiv\left(\rho, \rho u, \frac{1}{2}\left(\rho u^{2}+3 \rho \boldsymbol{T}\right)\right)^{t}$ and $\boldsymbol{F}(\overrightarrow{\boldsymbol{u}}) \equiv\left(\rho u, \rho u^{2}+\rho \boldsymbol{T}, \frac{1}{2} \rho u\left(u^{2}+5 \boldsymbol{T}\right)\right)^{t}$, the compressible Euler equation, (1.2), can be rewritten in a vector form:

$$
\partial_{t} \overrightarrow{\boldsymbol{u}}+\partial_{x} \boldsymbol{F}(\overrightarrow{\boldsymbol{u}})=0, \overrightarrow{\boldsymbol{u}} \in \mathbb{R}^{3} .
$$

The eigenvalues of $\boldsymbol{F}^{\prime}(\overrightarrow{\boldsymbol{u}})$,

$$
\boldsymbol{F}^{\prime}(\overrightarrow{\boldsymbol{u}})=\left(\begin{array}{ccc}
0 & 1 & 0 \\
-2 u^{2} / 3 & 4 u / 3 & 2 / 3 \\
-\left(15 \boldsymbol{T}+u^{2}\right) u / 6 & -u^{2} / 6 & 5 u / 3
\end{array}\right),
$$

define three Euler characteristics

$$
\left\{\lambda_{1}(\overrightarrow{\boldsymbol{u}}), \lambda_{2}(\overrightarrow{\boldsymbol{u}}), \lambda_{3}(\overrightarrow{\boldsymbol{u}})\right\}=\{u-\sqrt{5 \boldsymbol{T} / 3}, u, u+\sqrt{5 \boldsymbol{T} / 3}\},
$$


with the corresponding right eigenvector fields,

$$
\left\{\boldsymbol{r}_{1}(\overrightarrow{\boldsymbol{u}}), \boldsymbol{r}_{2}(\overrightarrow{\boldsymbol{u}}), \boldsymbol{r}_{3}(\overrightarrow{\boldsymbol{u}})\right\}=\left\{\left(\begin{array}{c}
\frac{\sqrt{15 \boldsymbol{T}} u+u^{2}}{2} \\
\frac{-\sqrt{15 \boldsymbol{T}}-2 u}{2} \\
1
\end{array}\right),\left(\begin{array}{c}
\frac{-5 \boldsymbol{T}+u^{2}}{2} \\
-u \\
1
\end{array}\right),\left(\begin{array}{c}
\frac{-\sqrt{15 \boldsymbol{T}} u+u^{2}}{2} \\
\frac{\sqrt{15 \boldsymbol{T}}-2 u}{2} \\
1
\end{array}\right)\right\},
$$

and left eigenvector fields, $\boldsymbol{l}_{i}(\overrightarrow{\boldsymbol{u}}), i=1,2,3$ :

$$
\begin{aligned}
& \boldsymbol{F}^{\prime}(\overrightarrow{\boldsymbol{u}}) \boldsymbol{r}_{i}(\overrightarrow{\boldsymbol{u}})=\lambda_{i}(\overrightarrow{\boldsymbol{u}}) \boldsymbol{r}_{i}(\overrightarrow{\boldsymbol{u}}), \\
& \boldsymbol{l}_{i}(\overrightarrow{\boldsymbol{u}}) \boldsymbol{F}^{\prime}(\overrightarrow{\boldsymbol{u}})=\lambda_{i}(\overrightarrow{\boldsymbol{u}}) \boldsymbol{l}_{i}(\overrightarrow{\boldsymbol{u}}), \\
& \boldsymbol{l}_{j}(\overrightarrow{\boldsymbol{u}}) \cdot \boldsymbol{r}_{i}(\overrightarrow{\boldsymbol{u}})=\delta_{i}^{j}, \quad i, j=1,2,3 .
\end{aligned}
$$

The main goal of this paper is to study the time-asymptotic stability of a Boltzmann shock profile $\varphi$, in the pointwise $L_{\xi, 3}^{\infty}$-norm:

$$
\left\{\begin{array}{l}
\partial_{t} F+\xi^{1} F_{x}-Q(F)=0 \text { for } t>0, x \in \mathbb{R} \\
\left\|(F(x, 0)-\varphi(x)) / \sqrt{\mathrm{M}_{-}}\right\|_{L_{\xi, 3}^{\infty}} \leq \delta \varepsilon e^{-4 \varepsilon|x| / D_{0}}
\end{array}\right.
$$

where the shock strength $\varepsilon$ and the magnitude of perturbation $\varepsilon \delta$ are assumed to satisfy

$$
\left\{\begin{array}{l}
\varepsilon \equiv\left|\rho_{-}-\rho_{+}\right|+\left|u_{-}-u_{+}\right|+\left|\boldsymbol{T}_{-}-\boldsymbol{T}_{+}\right| \ll 1, \\
\delta \ll \varepsilon^{1 / 2} /|\log \varepsilon| .
\end{array}\right.
$$

Here the constant $1 / D_{0}>0$ is a universal constant in determining the shock profile converging rate to its far fields

$$
\sup _{x \in \mathbb{R}}\left\|\varphi^{\prime}(x)\right\|_{L_{\xi, 3}^{\infty}} e^{\frac{4 \varepsilon|x|}{D_{0}}}<\infty,
$$

and the $L_{\xi, \beta}^{\infty}$-norm is defined by

$$
\|\mathbf{f}\|_{L_{\xi, \beta}^{\infty}} \equiv \sup _{\xi \in \mathbb{R}^{3}} \frac{|\mathbf{f}(\xi)|}{(1+|\xi|)^{\beta}}, \beta \geq 0 .
$$

Here and throughout the paper, the Knudsen number $\kappa$ is set to be

$$
\kappa=1 ;
$$

the only physical scale for the Cauchy problem is the mean-free path.

The main theorem of this paper is to describe the optimal pointwise convergence rate to the shock profile.

Theorem 1.1 (Main Theorem). Suppose that the strength $\varepsilon$ of a stationary 3-shock wave $\left(\overrightarrow{\boldsymbol{u}}_{-}, \overrightarrow{\boldsymbol{u}}_{+}\right)$is sufficiently small and that $\delta \ll \varepsilon^{1 / 2} /|\log \varepsilon|$.

Then there exist $x_{0}>0$ and $D_{1}>0$ such that the solution $F(x, t, \xi)$ of (1.6) satisfies:

Case 1. $t \in\left(0, \varepsilon^{-2}\right)$.

$$
\begin{aligned}
\left\|\frac{F(x, t)-\varphi\left(x-x_{0}\right)}{\sqrt{\mathrm{M}_{-}}}\right\|_{L_{\xi, 3}^{\infty}} \leq O(1) \delta \varepsilon\left(\sum_{j=1}^{2} e^{-\frac{\varepsilon\left|x-\lambda_{j}^{-} t\right|}{D_{1}}}+e^{-\frac{\varepsilon|x|}{D_{1}}}\right) \\
+O(1) \delta \varepsilon\left(\sum_{j=1}^{2} \frac{\chi_{\left[\lambda_{j}^{-} t, 0\right]}(x)}{\sqrt{\left|x-\lambda_{j}^{-} t\right|+1}}+\frac{\chi_{\left[\lambda_{j}^{-} t, 0\right]}(x)}{\sqrt{|x|+1}}\right) .
\end{aligned}
$$


Case 2. $t \geq \varepsilon^{-2}$.

$$
\begin{aligned}
& \left\|\frac{F(x, t, \cdot)-\varphi\left(x-x_{0}\right)}{\sqrt{\mathrm{M}_{-}}}\right\|_{L_{\xi, 3}^{\infty}} \\
& \leq O(1) \delta\left(\frac{\chi_{\left[\lambda_{1}^{-}(t+1)+\sqrt{t+1}, \lambda_{2}^{-}(t+1)-\sqrt{t+1}\right]}(x)}{\sqrt{\left|x-\lambda_{1}^{-} t\right|\left|x-\lambda_{2}^{-} t\right|}}+\frac{\chi_{\left[\lambda_{2}^{-}(t+1)+\sqrt{t+1}, 0\right]}(x)}{\sqrt{\left|x-\lambda_{2}^{-} t\right|\left|x-\lambda_{3}^{-} t\right|}}\right) \\
& +\delta\left(\sum_{j=1}^{2} \frac{e^{-\frac{\left(x-\lambda_{j}^{-} t\right)^{2}}{D_{0}(t+1)}}}{\sqrt{t+1}}+O(1) \frac{e^{-\frac{\varepsilon|x|}{D_{0}}-\frac{\varepsilon^{2} t}{E_{0}}}}{\sqrt{t+1}}+O(1) \varepsilon \frac{e^{-\frac{\varepsilon\left|x-\lambda_{1}^{-} t\right|}{4 D_{0}}}}{\sqrt{t+1}} \chi_{\left(-\infty, \lambda_{1}^{-} t-\sqrt{1+t]}\right.}(x)\right)
\end{aligned}
$$

where

$$
\left\{\begin{array}{l}
\left\{\lambda_{1}^{-}, \lambda_{2}^{-}, \lambda_{3}^{-}\right\} \equiv\left\{u_{-}-\sqrt{\frac{5 \boldsymbol{T}_{-}}{3}}, u_{-}, u_{-}+\sqrt{\frac{5 \boldsymbol{T}_{-}}{3}}\right\}, \\
\lambda_{3}^{-}=O(1) \varepsilon>0, \\
\chi_{[a, b]}: \text { the characteristic function of the interval }[a, b] .
\end{array}\right.
$$

Here, the constant $x_{0}$ is determined by the algebraic relationship:

$$
\int_{\mathbb{R}}\left(\begin{array}{c}
\left(1, F(x, 0, \cdot)-\varphi\left(x-x_{0}\right)\right) \\
\left(\xi^{1}, F(x, 0, \cdot)-\varphi\left(x-x_{0}\right)\right) \\
\left(\frac{1}{2}|\xi|^{2}, F(x, 0, \cdot)-\varphi\left(x-x_{0}\right)\right)
\end{array}\right) d x \in \operatorname{Span}\left\{\boldsymbol{r}_{1}\left(\overrightarrow{\boldsymbol{u}}_{-}\right), \boldsymbol{r}_{2}\left(\overrightarrow{\boldsymbol{u}}_{-}\right)\right\}
$$

and the vectors $\boldsymbol{r}_{i}\left(\overrightarrow{\boldsymbol{u}}_{-}\right)$are the left eigenvectors of the matrix $\boldsymbol{F}^{\prime}\left(\overrightarrow{\boldsymbol{u}}_{-}\right)$. When the shock $\left(\overrightarrow{\boldsymbol{u}}_{-}, \overrightarrow{\boldsymbol{u}}_{+}\right)$is a 1-shock wave, one replaces span $\left\{\boldsymbol{r}_{1}\left(\overrightarrow{\boldsymbol{u}}_{-}\right), \boldsymbol{r}_{2}\left(\overrightarrow{\boldsymbol{u}}_{-}\right)\right\}$in (1.9) with $\operatorname{span}\left\{\boldsymbol{r}_{2}\left(\overrightarrow{\boldsymbol{u}}_{+}\right), \boldsymbol{r}_{3}\left(\overrightarrow{\boldsymbol{u}}_{+}\right)\right\}$.

The time-asymptotic stability problem of a Boltzmann shock profile under the planar wave perturbation was first investigated in 12 in the study of the positivity of the Boltzmann shock profiles. There the stability analysis is obtained through the energy estimates under a zero total macroscopic mass condition,

$$
\int_{\mathbb{R}}\left(\psi_{i}, F(x, 0)-\varphi(x)\right) d x=0 \text { for } i=0,1,4,
$$

where $\psi_{i}$ are the collision invariants,

$$
\left\{\psi_{0}, \psi_{1}, \psi_{4}\right\} \equiv\left\{1, \xi^{1}, \frac{1}{2}|\xi|^{2}\right\} .
$$

The study in [12] is based on the energy method, which is motivated by the energy method in 2 for the stability of a viscous shock profile. The energy method is a basic techniques in the study of differential equations and works for the stability analysis of contact and rarefaction waves. However, it has not been shown to be sufficient for the shock wave studies in general. A Green's function pointwise estimate approach was initiated in [13, 14, for the purpose of better understanding the qualitative and quantitative behaviors of perturbations of a viscous shock profile. We generalize and refine this approach here to have a better handling of the local wave interactions. The energy estimates are too global for the understanding of these interactions. In [13] the Green's function of the Boltzmann equation linearized around a global Maxwellian was constructed with exponentially sharp pointwise structures. The structures of the Green's function show that, for 
instance, the primary fluid wave structure is a sum of transported heat kernels along each characteristic fields. With the detailed structure, one had obtained the pointwise nonlinear wave coupling structure around an absolute Maxwellian state.

There have been various successes in applying the Green's function around a global Maxwellian state as a basic tool to study various physical phenomena. For example, in [15] the time-asymptotic stability problem of a global Maxwellian in the half-space was resolved through the Green's function with global energy estimates. In a recent paper [17, nonlinear invariant manifolds for the steady Boltzmann flows are constructed through the Green's function approach. These invariant manifolds are the main instruments to study the monotonicity of a weak Boltzmann shock profile and the bifurcation problem on the condensation-evaporation problem proposed by Sone et al. in 19, 20, 21, 22. The Green's function of a linear Boltzmann equation captures the basic particle-like and wave-like structures in the solutions of the Boltzmann equation. It is useful for analyzing rather complex wave behavior. This paper is the first study of the Green's function for a variable coefficient problem. Previous studies based on Fourier transform work for constant coefficient problems, such as the Boltzmann equation linearized around a global Maxwellian. We use the Green's function around a global Maxwellian state previously studied and a compressive scalar-like equation to device a $\mathbb{T}-\mathbb{C}$ scheme to build the global wave structure. We now describe the scheme.

The $\mathbb{T}$ of the $\mathbb{T}-\mathbb{C}$ scheme stands for Transverse and Transient to far field. This can be identified as a hyperbolic wave structure at a given far field. The $\mathbb{C}$ stands for Compressive and Coupling with shock fronts. The $\mathbb{C}$ part will be identified as a local structure in the $x$-variable around a shock front but it is a global structure in the $t$-variable.

The prototype of the $\mathbb{T}-\mathbb{C}$ scheme begins with the wave propagation for the linearized Euler equations of a pulse around the stationary 3 -shock wave $\left(\overrightarrow{\boldsymbol{u}}_{-}, \overrightarrow{\boldsymbol{u}}_{+}\right)$: For a given pulse $\overrightarrow{\boldsymbol{v}}_{0} \delta(x)$ with $\overrightarrow{\boldsymbol{v}}_{0} \in \mathbb{R}^{3}$ initially, one can uniquely decompose $\overrightarrow{\boldsymbol{v}}_{0}$ into

$$
\overrightarrow{\boldsymbol{v}}_{0}=\sum_{j=1}^{2} v_{j} \boldsymbol{r}_{j}\left(\overrightarrow{\boldsymbol{u}}_{-}\right)+\frac{v_{3}}{\left|\overrightarrow{\boldsymbol{u}}_{-}-\overrightarrow{\boldsymbol{u}}_{+}\right|}\left(\overrightarrow{\boldsymbol{u}}_{-}-\overrightarrow{\boldsymbol{u}}_{+}\right) .
$$

This pulse will evolve into

$$
\overrightarrow{\boldsymbol{v}}(x, t)=\sum_{j=1}^{2} v_{j} \overrightarrow{\boldsymbol{r}}_{j}\left(\overrightarrow{\boldsymbol{u}}_{-}\right) \delta\left(x-\lambda_{j}\left(\overrightarrow{\boldsymbol{u}}_{-}\right) t\right)+v_{3} \frac{\overrightarrow{\boldsymbol{u}}_{-}-\overrightarrow{\boldsymbol{u}}_{+}}{\left|\overrightarrow{\boldsymbol{u}}_{-}-\overrightarrow{\boldsymbol{u}}_{+}\right|} \delta(x) .
$$

The pulse satisfies the linearized Euler equation around the shock wave $\left(\overrightarrow{\boldsymbol{u}}_{-}, \overrightarrow{\boldsymbol{u}}_{+}\right)$ for $x \neq 0$ and the conservation laws

$$
\int_{\mathbb{R}} \overrightarrow{\boldsymbol{v}}(x, t) d x=\overrightarrow{\boldsymbol{v}}_{0}
$$

for all $t>0$. The component $\overrightarrow{\boldsymbol{v}}^{t}(x, 0) \equiv\left(v_{1} \overrightarrow{\boldsymbol{r}}_{1}\left(\overrightarrow{\boldsymbol{u}}_{-}\right)+v_{2} \overrightarrow{\boldsymbol{r}}_{2}\left(\overrightarrow{\boldsymbol{u}}_{-}\right)\right) \delta(x)$ in $\overrightarrow{\boldsymbol{v}}_{0}$ and the linearized equation

$$
\partial_{t} \overrightarrow{\boldsymbol{v}}^{t}+\boldsymbol{F}^{\prime}\left(\overrightarrow{\boldsymbol{u}}_{-}\right) \partial_{x} \overrightarrow{\boldsymbol{v}}^{t}=0
$$

form the prototype for wave propagation at the far field. This is for the $\mathbb{T}$ part. 
In (1.12), there is a component $v_{3}\left(\overrightarrow{\boldsymbol{u}}_{+}-\overrightarrow{\boldsymbol{u}}_{-}\right) \delta(x) /\left|\overrightarrow{\boldsymbol{u}}_{+}-\overrightarrow{\boldsymbol{u}}_{-}\right|$which is stacked at the shock front due to the compressibility of the shock wave. Waves in this stacked component are confined around the shock front.

Since only the Rankine-Hugoniot condition for the far field is used, the structure of the Boltzmann shock $\varphi$ does not register in the pulse $\overrightarrow{\boldsymbol{v}}(x, t)$. This pulse should not be refined enough to resolve the structure around the shock front. In contrast to this, the construction of the $\mathbb{C}$ part will require the essential information on the profile $\varphi$, and it preserves the localized structure around the shock front as well as the macroscopic conservation laws. To fit these criteria, one introduces a local wave front tracing and the linearized Boltzmann equation around a shock profile with a small lower damping to form the $\mathbb{C}$ part.

With the above hyperbolic wave prototype, one sets up the operators $\mathbb{T}, \mathbb{D}, \mathbb{R}_{3}$, and $\mathbb{C}_{3}$ for the linearized Boltzmann equation around the Boltzmann shock profile $\varphi$

$$
\partial_{t} \mathrm{~h}+\xi^{1} \partial_{x} \mathrm{~h}-\mathrm{L}_{\varphi} \mathrm{h}=0, \mathrm{~L}_{\varphi} \mathrm{h} \equiv \mathrm{M}_{-}^{-1 / 2} \mathrm{~B}\left(\varphi, \mathrm{M}_{-}^{1 / 2} \mathrm{~h}\right)
$$

as follows:

First, corresponding to the decomposition (1.11) one uses the collision invariants $\left\{\psi_{0} \sqrt{\mathrm{M}_{-}}, \psi_{1} \sqrt{\mathrm{M}_{-}}, \psi_{4} \sqrt{\mathrm{M}_{-}}\right\}$to define the transverse macroscopic components, the compressive macroscopic component, and the microscopic components of the function $\mathrm{u} \in L_{\xi}^{2}$.

Definition 1.2 (Macroscopic Hyperbolic Wave Decomposition).

$$
\begin{cases}\mathrm{u}^{t} \equiv\left(\mathrm{I}_{1}, \mathrm{u}\right) \mathrm{r}_{1}+\left(\mathrm{I}_{2}, \mathrm{u}\right) \mathrm{r}_{2} & \text { (Transverse Component) } \\ \mathrm{u}^{c} \equiv\left(\mathrm{I}_{3}, \mathrm{u}\right) \mathrm{r}_{3} & \text { (Compressive Component) } \\ \mathrm{u}^{m} \equiv \mathrm{u}-\mathrm{u}^{t}-\mathrm{u}^{c} & \text { (Microscopic Component) }\end{cases}
$$

where $\mathrm{I}_{i}, \mathrm{r}_{j} \in \operatorname{Span}\left\{\psi_{0} \sqrt{\mathrm{M}_{-}}, \psi_{1} \sqrt{\mathrm{M}_{-}}, \psi_{4} \sqrt{\mathrm{M}_{-}}\right\}$satisfies

$$
\left\{\begin{array}{l}
\left(\begin{array}{c}
\left(\psi_{0}, \mathrm{r}_{j}\right) \\
\left(\psi_{1}, \mathrm{r}_{j}\right) \\
\left(\psi_{4}, \mathrm{r}_{j}\right)
\end{array}\right)=\overrightarrow{\boldsymbol{r}}_{j}\left(\overrightarrow{\boldsymbol{u}}_{-}\right) \text {for } j=1,2,\left(\begin{array}{c}
\left(\psi_{0}, \mathrm{r}_{3}\right) \\
\left(\psi_{1}, \mathrm{r}_{3}\right) \\
\left(\psi_{4}, \mathrm{r}_{3}\right)
\end{array}\right)=\frac{\overrightarrow{\boldsymbol{u}}_{+}-\overrightarrow{\boldsymbol{u}}_{-}}{\left|\overrightarrow{\boldsymbol{u}}_{+}-\overrightarrow{\boldsymbol{u}}_{-}\right|}, \\
\left(\mathrm{I}_{j}, \mathrm{r}_{i}\right)=\delta_{j}^{i},
\end{array}\right.
$$

where the vector $\overrightarrow{\boldsymbol{r}}_{j}\left(\overrightarrow{\boldsymbol{u}}_{-}\right)$is given in (1.4) and $\overrightarrow{\boldsymbol{u}}_{ \pm}=\left(\rho_{ \pm}, \rho_{ \pm} u_{ \pm}, \rho_{ \pm}\left(\left|u_{ \pm}\right|^{2}+3 \boldsymbol{T}_{ \pm}\right) / 2\right)$.

Referring to (1.13), the $\mathbb{T}$ operator is an operator for any given inhomogeneous term ER of (1.14) with the property $\|\mathrm{ER}\|_{L_{\xi, 2}^{\infty}} \leq O(1) e^{-\varepsilon|x| / D_{1}}$. The function $\mathbb{T}[\mathrm{ER}](x, t)$ is constructed to satisfy

$$
\left\{\begin{array}{l}
\mathbb{T}[\mathrm{ER}](x, 0) \equiv 0, \\
\left(\mathrm{I}_{j},\left(\partial_{t}+\xi^{1} \partial_{x}-\mathrm{L}_{\varphi}\right) \mathbb{T}[\mathrm{ER}](x, t)-\operatorname{ER}(x, t)\right)=0 \text { for } j=1,2, \\
\sup _{x \in \mathbb{R}}\left\|\left(\partial_{t}+\xi^{1} \partial_{x}-\mathrm{L}_{\varphi}\right) \mathbb{T}[\mathrm{ER}](x, t)\right\|_{L_{\xi, 2}^{\infty}} e^{\varepsilon|x| / D_{1}}<\infty .
\end{array}\right.
$$

The main tool for the construction is the Green's function of the constant coefficient problem at the left far field,

$$
\left\{\begin{array}{l}
\left(\partial_{t}+\xi^{1} \partial_{x}-\mathrm{L}_{-}\right) \mathrm{t}=\mathrm{ER}^{t} \\
\mathrm{t}(x, 0) \equiv 0
\end{array}\right.
$$


where $L_{-} t \equiv \sqrt{M_{-}^{-1}} B\left(M_{-}, \sqrt{M_{-}} t\right)$. The pointwise structure of the Green's function $\mathbb{G}_{-}$for the linearized Boltzmann equation around the state $M_{-}$plays an important role in obtaining the exponential space decaying structure. This is a strong localization in $x$ property needed to understand the nature of the scale separation.

In (1.17), the macroscopic conservation laws in the $r_{3}$-component is not valid. One needs to adjust the errors in the compressive field by introducing a local wave front tracing, $\mathbb{D}[\mathrm{ER}]$ :

$$
\left\{\begin{array}{l}
\mathbb{D}[\mathrm{ER}](x, t) \equiv d(t) \frac{\varphi^{\prime}(x)}{\left(\mathrm{I}_{3},\left(\mathrm{M}_{+}-\mathrm{M}_{-}\right) / \sqrt{\mathrm{M}_{-}}\right)}, \\
d(t) \equiv \int_{0}^{t} \int_{\mathbb{R}}\left(\mathrm{I}_{3},-\left(\partial_{t}+\xi^{1} \partial_{x}-\mathrm{L}_{\varphi}\right) \mathbb{T}[\mathrm{ER}](x, \tau)+\operatorname{ER}(x, \tau)\right) d x d \tau .
\end{array}\right.
$$

With this, all the macroscopic conservation laws are satisfied

$$
\int_{\mathbb{R}}\left(\mathrm{I}_{j},\left(\partial_{t}+\xi^{1} \partial_{x}-\mathrm{L}_{\varphi}\right)(\mathbb{T}+\mathbb{D})[\mathrm{ER}](x, t)-\mathrm{ER}(x, t)\right) d x=0 \text { for } t \geq 0, j=1,2,3 .
$$

Set

$$
\mathbb{R}_{3}[\mathrm{ER}](x, t) \equiv \operatorname{ER}(x, t)-\left(\partial_{t}+\xi^{1} \partial_{x}-\mathrm{L}_{\varphi}\right)(\mathbb{T}+\mathbb{D})[\mathrm{ER}](x, t) .
$$

This is the residue component for the interaction between the $\mathbb{T}$-component and the shock profile. One introduces a compressive operator $\mathbb{C}_{3}\left[\mathbb{R}_{3}[\mathrm{ER}]\right]$ to monitor the interaction of the residue function $\mathbb{R}_{3}[E R]$. The defining function $\mathrm{C}(x, t)$ for $\mathbb{R}_{3}[\mathrm{ER}](x, t)$ is

$$
\begin{aligned}
& \left\{\begin{array}{l}
\left(\partial_{t}+\xi^{1} \partial_{x}-\mathrm{L}_{\varphi}\right) \mathrm{C}-\mathbb{R}_{3}[\mathrm{ER}]=-\varepsilon \sum_{j=1}^{2}\left(\mathrm{I}_{j}, \mathrm{C}\right) \mathrm{r}_{j}, \\
\mathrm{C}(x, 0) \equiv 0
\end{array}\right. \\
& \mathbb{C}_{3}\left[\mathbb{R}_{3}[\mathrm{ER}]\right](x, t) \equiv \mathrm{C}(x, t) .
\end{aligned}
$$

This yields $\frac{d}{d t} \int_{\mathbb{R}}\left(\mathrm{I}_{k}, \mathrm{C}(x, t)\right) d x=-\sum_{j=1}^{2} \delta_{j}^{k} \int_{\mathbb{R}}\left(\mathrm{I}_{k}, \mathrm{C}(x, t)\right) d x$ and $\int_{\mathbb{R}}\left(\mathrm{I}_{k}, \mathrm{C}(x, 0)\right) d x=$ 0 for $k=1,2,3$. Thus, the function $\mathrm{C}(x, t)$ preserves the macroscopic conservation laws,

$$
\int_{\mathbb{R}}\left(\mathrm{I}_{j}, \mathrm{C}(x, t)\right) d x=0 \text { for } j=1,2,3 \text { and } t>0 .
$$

In (1.21), the term $\sum_{j=1}^{2}\left(\mathrm{I}_{j}, \mathrm{C}\right) \mathrm{r}_{j}$ is almost virtual, since the transverse component in $\mathbb{R}_{3}[\mathrm{ER}]$ is small. This almost virtual term $\sum_{j=1}^{2}\left(\mathrm{I}_{j}, \mathrm{C}\right) \mathrm{r}_{j}$ can therefore be damped in (1.21) with small truncation error. With this damping term, there is an exponential decay structure in the solution $\mathrm{C}(x, t)$; and one can have global exponential estimates on the solution by energy estimates. To perform the exponential decaying rate through the energy estimates, the compressibility property of the Boltzmann shock profile $\varphi$ is used to obtain the exponential rate by the energy method. The compressibility of $\varphi$ is the essential nonlinearity information. 
Finally, one defines an operator $\mathrm{T}[\mathrm{ER}]$ to approximate the solution $\left(\partial_{t}+\xi^{1} \partial_{x}-\right.$ $\left.\mathrm{L}_{\varphi}\right) \mathrm{h}-\mathrm{ER}=0$ with $\mathrm{h}(x, 0) \equiv 0$ and its truncation error $\mathfrak{R}[\mathrm{ER}]$ :

$$
\left\{\begin{array}{l}
\mathrm{T}[\mathrm{ER}](x, t) \equiv\left(\mathbb{T}+\mathbb{D}+\mathbb{C}_{3}\left[\mathbb{R}_{3}\right]\right)[\mathrm{ER}](x, t), \\
\mathfrak{R}[\mathrm{ER}](x, t) \equiv \varepsilon \sum_{j=1}^{2}\left(\mathrm{I}_{j}, \mathbb{C}_{3}\left[\mathbb{R}_{3}[\mathrm{ER}]\right](x, t)\right) \mathrm{r}_{j},
\end{array}\right.
$$

and

$$
\left(\partial_{t}+\xi^{1} \partial_{x}-\mathrm{L}_{\varphi}\right)\left(\mathbb{T}+\mathbb{D}+\mathbb{C}_{3}\left[\mathbb{R}_{3}\right]\right)[\mathrm{ER}]-\mathrm{ER}=-\mathfrak{R}[\mathrm{ER}] .
$$

Here, to verify that $\mathfrak{R}[\mathrm{ER}]$ is small with localized structure in the $x$-variable, the sharp pointwise structures of the Green's functions for $\left(\partial_{t}+\xi^{1} \partial_{x}-\mathrm{L}_{-}\right) \mathrm{h}=0$, $\left(\partial_{t}+\xi^{1} \partial_{x}-\mathrm{L}_{+}\right) \mathrm{h}=0$, and $\left(\partial_{t}+\xi^{1} \partial_{x}-\mathrm{L}_{-}\right) \mathrm{h}+\varepsilon \sum_{j=1}^{2}\left(\mathrm{I}_{j}, \mathrm{~h}\right) \mathrm{r}_{j}=0$, and the energy estimates for $\left(\partial_{t}+\xi^{1} \partial_{x}-\mathrm{L}_{\varphi}\right) \mathrm{h}+\varepsilon \sum_{j=1}^{2}\left(\mathrm{I}_{j}, \mathrm{~h}\right) \mathrm{r}_{j}=0$ are combined together.

The smallness and pointwise localization property of $\mathfrak{R}[\mathrm{ER}]$ will assure that the series $\sum_{j=1}^{\infty} \mathrm{T}\left[\mathfrak{R}_{k}[\mathrm{ER}]\right]$ obtained by the $\mathbb{T}-\mathbb{C}$ scheme converges where

$$
\left\{\begin{array}{l}
\mathfrak{R}_{1}[\mathrm{ER}]=\mathrm{ER}, \\
\mathfrak{R}_{k}[\mathrm{ER}]=\mathfrak{R}\left[\mathfrak{R}_{k-1}[\mathrm{ER}]\right] \text { for } k \geq 2 .
\end{array}\right.
$$

This series solves

$$
\left(\partial_{t}+\xi^{1} \partial_{x}-\mathrm{L}_{\varphi}\right) \sum_{j=1}^{\infty} \mathrm{T}\left[\Re_{k}[\mathrm{ER}]\right]=\mathrm{ER} .
$$

We now summarize the key components in constructing the operators $\mathbb{T}$ in (1.17), $\mathbb{C}_{3}$ in (1.21), $\mathbb{D}$ in (1.18), and their basic features. The exponentially sharp pointwise structures in the Green's functions $\mathbb{G}_{ \pm}$of the linearized equations, $\left(\partial_{t}+\xi^{1} \partial_{x}-\right.$ $\left.\mathrm{L}_{ \pm}\right) \mathrm{f}_{ \pm}=0$, around constant Maxwellian states are important for separating the scale in the space variable so that the exponential decaying structures ensure the convergence of the iterations based on the operator $\mathbb{T}$ and $\mathfrak{R}_{k}$. The role of the $\mathbb{T}$ operator is to extract the transverse components towards the far field. The lower order damping terms in constructing the compressive wave operator $\mathbb{C}_{3}$ play a role in filtering the compressive component and in confining it around the shock front for all time. However, to realize this mechanism, the combinations of the Green's function and the energy estimates are necessary. The energy estimates give global $L_{x}^{2}\left(L_{\xi}^{2}\right)$ estimates with exponential growth weighted functions; and the Green's function refines the global estimates into pointwise estimates. This is the parallel process introduced in this paper. The operator $\mathbb{D}$ for the local wave front tracing plays the role of preserving the macroscopic conservation laws. These operators, developed to separate variable scales and to construct the Green's function for variable coefficient problems, should be useful for future intended study of establishing the interactions between initial layers and shock layers as well as the Riemann problem for the Boltzmann equation.

In Section 2, we review the necessary materials for this paper from both hyperbolic conservation laws and Boltzmann equations. In particular, the fine structure of the Green's function of a linearized Boltzmann equation around a global Maxwellian is reviewed in detail.

In Section 3, we make a comparison of the pointwise structures of the Green's functions related to the linearized Boltzmann equations around the different far field 
Maxwellian states of a Boltzmann shock layer as the preparation for the analysis in Section 5.

In Section 4, we use the Green's functions of the linearized Boltzmann equations around the Maxwellian states associated with the end states of a stationary 3-shock wave $\left(\overrightarrow{\boldsymbol{u}}_{-}, \overrightarrow{\boldsymbol{u}}_{+}\right)$to define the scheme for building the transverse wave operator $\mathbb{T}$. We introduce a linear local wave front tracing operator $\mathbb{D}$ and a residue operator $\mathbb{R}_{3}$. A compressive wave operator $\mathbb{C}_{3}$, which is the linearized Boltzmann equation around a Boltzmann shock profile with an upwind damping term, was established. We introduce a Transverse-Compressive scheme $(\mathbb{T}-\mathbb{C}$ scheme) in terms of the operators $\mathbb{T}, \mathbb{C}_{3}, \mathbb{D}$, and $\mathbb{R}_{3}$ to study an initial value problem of a linearized Boltzmann equation around the Boltzmann shock profile.

Section 5 studies the estimates of the operators $\mathbb{T}, \mathbb{C}_{3}, \mathbb{D}$, and $\mathbb{R}_{3}$. The pointwise estimates on the $\mathbb{C}_{3}$ operator require a combination of energy estimates and the Green's function of an upwind damped Boltzmann equation linearized around the left end state of a 3-shock wave. Here the energy estimates are global estimates in the space-time variables for wave interactions in the compressive field only. In particular, with the choice of an exponential growth weighted function in the $x$ variable, the energy estimates possess an exponential decaying rate in the time variable. This time exponential decaying structure is due to a combination of the damping effect in the transverse field and the nondegenerated compressive field.

In Section 6, we prove the convergence of the $\mathbb{T}-\mathbb{C}$ scheme and establish the wave propagation patterns for waves emitting from the center of a shock layer. With this wave propagation structure, one obtains the wave scattering patterns around the front of the Boltzmann shock profile. This is a key element for the study of the nonlinear wave coupling in Section 7. Finally, in Section 7 we make an ansatz assumption of the nonlinear wave propagation and use the estimates obtained in Section 6 to prove the ansatz assumption and obtain the main theorem.

We collect all computational lemmas in the appendix. Most of the lemmas in the appendix were obtained by Liu $[8]$ in the study on the time-asymptotic convergence to a shock profile for a system of viscous conservation laws with an artificial viscosity matrix. The paper 8 had initiated the study of the large time coupling of nonlinear waves. Recently, 10, has established a completion on the viscous shock stability along the framework of $[8$.

The basic philosophy in this paper is different from that in 8 . The $\mathbb{T}-\mathbb{C}$ scheme allows for more accurate and local study of wave behavior. The difference lies in the anti-derivative variables for the conservation laws. The anti-derivative variables in [8] are for the full nonlinear system, but the ones in this paper are for the linearized system. Since the anti-derivative variables are global variables, it is difficult to recover the full local structure in $x$ from the anti-derivative variables of a full nonlinear system. In this paper, the anti-derivative variables were introduced only for the compressive operator $\mathbb{C}_{3},(1.21)$. This operator $\mathbb{C}_{3}$ is already a local operator in $x$, and its anti-derivative variables are local too. Thus, the $\mathbb{T}-\mathbb{C}$ scheme can obtain a fully local structure to study the nonlinear coupling. In [23, 9, the spectral analysis and complex analysis were introduced to yield the exponentially sharp structure of the Green's function for problems around a constant state such as the compressible Navier-Stokes equation. It provides a tool for the development of the Green's functions in 13, 14, which are parts of the basic tools in this paper. About 
the local dynamics $\mathbb{D}$, there were various previous works $[3,11$, 16] to motivate its development.

The Green's function for a general 3-D perturbation constructed in [14 would be a useful instrument to study wave propagation in multi-D problems such as the stability of a planar shock profile under a small 3-D perturbation. This is, however, left for the future.

\section{Preliminaries}

This paper considers planar wave solutions $F(x, t, \xi)$ in the $x$-direction. The symmetries in $\xi^{2}$ and $\xi^{3}$ are imposed:

$$
F\left(x, t, \xi^{1}, \xi^{2}, \xi^{3}\right)=F\left(x, t, \xi^{1},-\xi^{2}, \xi^{3}\right)=F\left(x, t, \xi^{1}, \xi^{2},-\xi^{3}\right)
$$

for all $(x, t, \xi) \in \mathbb{R} \times[0, \infty) \times \mathbb{R}^{3}$.

In this section we review the basics of hyperbolic conservation laws and the basic theories on the linear and nonlinear collision operators for a hard sphere collision model for the Boltzmann equation, the macro-micro decomposition, and the Green's function of the linearized equation around an absolute Maxwellian state.

2.1. Basic theories on hyperbolic conservation laws. The hyperbolic system (1.3) gives $n(n=3)$ linearly independent vector fields, $\overrightarrow{\boldsymbol{r}}_{j}(\overrightarrow{\boldsymbol{u}}), j=1, \cdots, n$, on the phase space $\overrightarrow{\boldsymbol{u}} \in \mathbb{R}^{n}$. Each characteristic field $\overrightarrow{\boldsymbol{r}}_{j}(\overrightarrow{\boldsymbol{u}})$ gives a family of integral curves, and we denote by $R_{j}\left(\overrightarrow{\boldsymbol{u}}_{0}\right)$ the integral curve through the point $\overrightarrow{\boldsymbol{u}}_{0} \in \mathbb{R}^{n}$. One can parametrize this integral curve by $\lambda_{j}(\overrightarrow{\boldsymbol{u}})$ when the characteristic field $\lambda_{j}$ $(j=1,3)$ is genuinely nonlinear, i.e. $\nabla_{\overrightarrow{\boldsymbol{u}}} \lambda_{j}(\overrightarrow{\boldsymbol{u}}) \neq 0$ :

$$
\begin{aligned}
R_{j}^{ \pm}\left(\overrightarrow{\boldsymbol{u}}_{0}\right) \equiv\left\{\mathfrak{R}(s) \mid \boldsymbol{F}^{\prime}(\mathfrak{R}(s)) \frac{d \mathfrak{R}(s)}{d s}=\right. & \lambda_{j}(\mathfrak{R}(s)) \frac{d \mathfrak{R}(s)}{d s}, s=\lambda_{j}(\mathfrak{R}(s)), \\
& \left. \pm\left(s-\lambda_{j}\left(\overrightarrow{\boldsymbol{u}}_{0}\right)\right) \geq 0, \mathfrak{R}_{j}\left(\lambda_{j}\left(\overrightarrow{\boldsymbol{u}}_{0}\right)\right)=\overrightarrow{\boldsymbol{u}}_{0},\right\} .
\end{aligned}
$$

We also denote by $H_{j}\left(\overrightarrow{\boldsymbol{u}}_{0}\right)$ the $j$-th Hugoniot curve connecting $\overrightarrow{\boldsymbol{u}}_{0}$ :

$$
H_{j}^{ \pm}\left(\overrightarrow{\boldsymbol{u}}_{0}\right) \equiv\left\{\mathfrak{H}(s) \mid s\left(\mathfrak{H}(s)-\overrightarrow{\boldsymbol{u}}_{0}\right)=\boldsymbol{F}(\mathfrak{H}(s))-\boldsymbol{F}\left(\overrightarrow{\boldsymbol{u}}_{0}\right), \pm\left(s-\lambda_{j}\left(\overrightarrow{\boldsymbol{u}}_{0}\right)\right)>0\right\} .
$$

Proposition 2.1. The states $\left(\overrightarrow{\boldsymbol{u}}_{-}, \overrightarrow{\boldsymbol{u}}_{+}\right)$can be connected by an $i$-th shock wave if and only if

$$
\overrightarrow{\boldsymbol{u}}_{+} \in H_{i}^{-}\left(\overrightarrow{\boldsymbol{u}}_{-}\right),
$$

and the states $\left(\overrightarrow{\boldsymbol{u}}_{-}, \overrightarrow{\boldsymbol{u}}_{+}\right)$can be connected by a rarefaction wave if and only if

$$
\overrightarrow{\boldsymbol{u}}_{+} \in R_{i}^{+}\left(\overrightarrow{\boldsymbol{u}}_{-}\right) .
$$

Proposition 2.2 (Liu). The integral curve $R_{i}\left(\overrightarrow{\boldsymbol{u}}_{0}\right)$ and the Hugoniot curve $H_{i}\left(\overrightarrow{\boldsymbol{u}}_{0}\right)$ are second order tangential to each other at $\overrightarrow{\boldsymbol{u}}_{0} ; c f$. [7].

This proposition yields that

$$
\left|\frac{\boldsymbol{F}\left(\overrightarrow{\boldsymbol{u}}_{-}\right)-\boldsymbol{F}\left(\overrightarrow{\boldsymbol{u}}_{+}\right)}{\left|\boldsymbol{F}\left(\overrightarrow{\boldsymbol{u}}_{-}\right)-\boldsymbol{F}\left(\overrightarrow{\boldsymbol{u}}_{+}\right)\right|}-\frac{\boldsymbol{r}_{i}\left(\overrightarrow{\boldsymbol{u}}_{-}\right)}{\left|\boldsymbol{r}_{i}\left(\overrightarrow{\boldsymbol{u}}_{-}\right)\right|}\right| \leq O(1)\left|\overrightarrow{\boldsymbol{u}}_{-}-\overrightarrow{\boldsymbol{u}}_{+}\right| \text {when }\left|\overrightarrow{\boldsymbol{u}}_{-}-\overrightarrow{\boldsymbol{u}}_{+}\right| \ll 1 .
$$




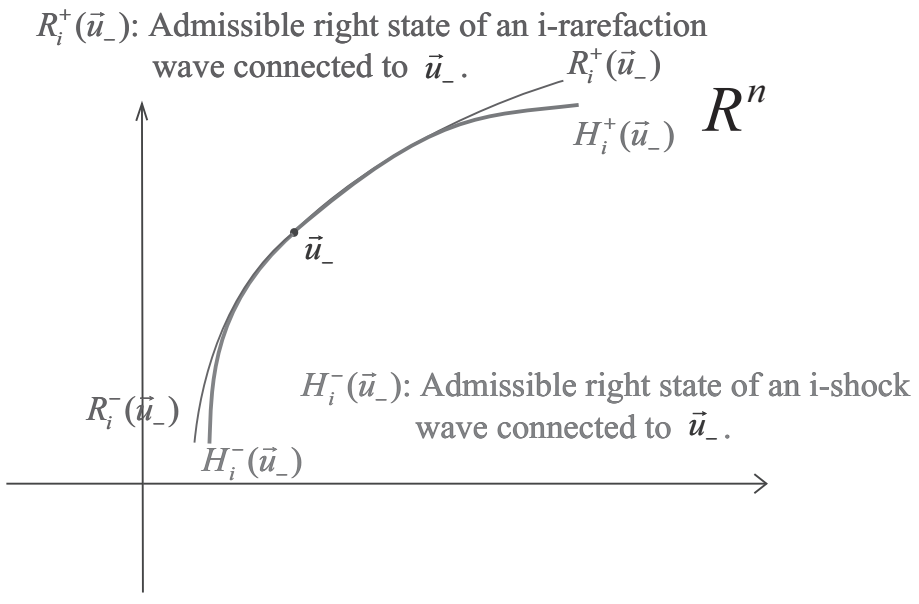

Figure 1. An illustration of the 2nd order tangential property of Hugoniot curves and integral curves.

2.2. Basic theories for the Boltzmann equation on the linear and nonlinear collision operators. In this subsection we review the basic theories on the collision operators for the hard sphere collision model.

Theorem 2.3 (Boltzmann, 1872).

$$
\begin{aligned}
& Q\left(\mathrm{M}_{[\rho, \boldsymbol{u}, \boldsymbol{T}]}\right)(\xi) \equiv 0 \text { for any }(\rho, \boldsymbol{u}, \boldsymbol{T}) \in \mathbb{R}^{+} \times \mathbb{R}^{3} \times \mathbb{R}^{+}, \\
& \int_{\mathbb{R}^{3}} \psi_{j}(\xi) Q(H)(\xi) d \xi=0 \text { for } j=0,1,2,3,4
\end{aligned}
$$

where $\psi_{j}$ are the collision invariants

$$
\begin{aligned}
& \left\{\psi_{0}, \psi_{1}, \psi_{2}, \psi_{3}, \psi_{4}\right\}=\left\{1, \xi^{1}, \xi^{2}, \xi^{3}, \frac{1}{2}|\xi|^{2}\right\}, \\
& \mathbf{M}_{[\rho, \boldsymbol{u}, \boldsymbol{T}]}(\xi) \equiv \rho \frac{e^{-\frac{|\xi-\boldsymbol{u}|^{2}}{2 \boldsymbol{T}}}}{(2 \pi \boldsymbol{T})^{3 / 2}}, \boldsymbol{u}, \xi \equiv\left(\xi^{1}, \xi^{2}, \xi^{3}\right) \in \mathbb{R}^{3} .
\end{aligned}
$$

Denote by $L$ the linearized collision operator around the absolute Maxwellian state:

$$
\left\{\begin{array}{l}
\mathrm{M}_{0} \equiv \mathrm{M}_{[1,0,1]}, \\
\operatorname{Lg}(\xi) \equiv \frac{1}{\sqrt{\mathrm{M}_{0}}} \mathrm{~B}\left(\mathrm{M}_{0}, \sqrt{\mathrm{M}_{0}} \mathrm{~g}\right) .
\end{array}\right.
$$

Theorem 2.4 (Hilbert). The linearized collision operator $\mathrm{L}$ can be decomposed as a sum of a multiplication operator and an integral operator:

$$
\begin{aligned}
& \operatorname{Lh}(\xi)=\nu(\xi) \mathrm{h}(\xi)+\int_{\mathbb{R}^{3}} K\left(\xi, \xi_{*}\right) \mathrm{h}\left(\xi_{*}\right) d \xi_{*}, \\
& \left\{\begin{array}{l}
\nu \equiv \frac{1}{\sqrt{2 \pi}}\left(2 e^{-\frac{|\xi|^{2}}{2}}+2\left(|\xi|+\frac{1}{|\xi|}\right) \int_{0}^{|\xi|} e^{-\frac{u^{2}}{2}} d u\right), \nu(\xi) \sim 1+|\xi|, \\
K\left(\xi, \xi_{*}\right) \equiv \frac{2}{\sqrt{2 \pi}\left|\xi-\xi_{*}\right|} \exp \left(-\frac{\left(|\xi|^{2}-\left|\xi_{*}\right|^{2}\right)^{2}}{8\left|\xi-\xi_{*}\right|^{2}}-\frac{\left|\xi-\xi_{*}\right|^{2}}{8}\right) \\
-\frac{\left|\xi-\xi_{*}\right|}{2} \exp \left(-\frac{\left(|\xi|^{2}+\left|\xi_{*}\right|^{2}\right)}{4}\right) .
\end{array}\right.
\end{aligned}
$$


Here the notion $(\cdot, \cdot)$ is the standard inner product of $L^{2}\left(\mathbb{R}^{3}\right) \cap\{\mathrm{f}:$ an even function in $\xi^{2}$ and $\xi^{3}$ variables\}: For any $\mathrm{g}$ and $\mathrm{h}$,

$$
\left\{\begin{array}{l}
(\mathrm{g}, \mathrm{h}) \equiv \int_{\mathbb{R}^{3}} \mathrm{~g}(\xi) \mathrm{h}(\xi) d \xi \\
\|\mathrm{g}\|_{L_{\xi}^{2}} \equiv\left(\int_{\mathbb{R}^{3}} \mathrm{~g}(\xi)^{2} d \xi\right)^{1 / 2} .
\end{array}\right.
$$

The weighted supernorm $\|\cdot\|_{L_{\xi, \alpha}^{\infty}}$ with $\alpha \geq 0$ is

$$
\left\{\begin{array}{l}
\|\mathrm{g}\|_{L_{\xi, \alpha}^{\infty}} \equiv \sup _{\xi \in \mathbb{R}^{3}}(1+|\xi|)^{\alpha}|\mathrm{g}(\xi)| \text { for } \alpha \geq 0, \\
\|\mathrm{~g}\|_{L_{\xi}^{\infty}} \equiv\|\mathrm{g}\|_{L_{\xi, 0}^{\infty}}^{\infty} .
\end{array}\right.
$$

Theorem 2.5 (Carleman). There exists $\nu_{0}>0$ such that for any $\mathrm{g}$ satisfying $\left(\psi_{j} \sqrt{\mathbf{M}_{0}}, \mathrm{~g}\right)=0$ for any $j=0,1,2,3,4$,

$$
(\mathrm{g}, \mathrm{Lg}) \leq-\nu_{0}\|\mathrm{~g}\|_{L_{\xi}^{2}}^{2} .
$$

Theorem 2.6 (Liu-Yu). There exists $\nu_{1}>0$ such that for any g satisfying $\left(\psi_{j} \sqrt{\mathrm{M}_{0}}, \mathrm{~g}\right)$ $=0$ for any $j=0,1,2,3,4$, [12]:

$$
(\mathrm{g}, \mathrm{Lg}) \leq-\nu_{1}\left\|(1+|\xi|)^{\frac{1}{2}} \mathrm{~g}\right\|_{L_{\xi}^{2}}^{2}
$$

2.3. Macro-micro decompositions around a hydrodynamic macroscopic state $\overrightarrow{\boldsymbol{v}}$. Referring to a given macroscopic state $(\varrho, v, \theta)$, a macro-micro decomposition $\left(\mathrm{P}_{0}^{\vec{v}}, \mathrm{P}_{1}^{\vec{v}}\right)$ is defined as follows:

$$
\begin{aligned}
& \overrightarrow{\boldsymbol{v}} \equiv\left(\varrho, \varrho v, \varrho\left(|v|^{2}+3 \theta\right) / 2\right) \text { (conservative variables), } \\
& \mathrm{M} \equiv \mathrm{M}_{[1, v, \theta]} \text {, } \\
& \mathrm{P}_{0}^{\vec{v}} \mathrm{~g} \equiv\left[\left(\mathrm{M}^{\frac{1}{2}}, \mathrm{~g}\right)+\left(\frac{\xi^{1}-v}{\sqrt{\theta}} \mathrm{M}^{\frac{1}{2}}, \mathrm{~g}\right) \frac{\xi^{1}-v}{\sqrt{\theta}}\right. \\
& \left.+\frac{1}{6}\left(\left(\frac{\left|\xi^{1}-v\right|^{2}+\left|\xi^{2}\right|^{2}+\left|\xi^{3}\right|^{2}}{\theta}-3\right) \mathrm{M}^{\frac{1}{2}}, \mathrm{~g}\right)\left(\frac{\left|\xi^{1}-v\right|^{2}+\left|\xi^{2}\right|^{2}+\left|\xi^{3}\right|^{2}}{\theta}-3\right)\right] \mathrm{M}^{\frac{1}{2}}, \\
& \mathrm{P}_{1}^{\vec{v}} \mathrm{~g} \equiv \mathrm{g}-\mathrm{P}_{0}^{\vec{v}} \mathrm{~g} \text {. }
\end{aligned}
$$

The Euler characteristics of the macroscopic components, Range $\left(\mathrm{P}_{0}^{\vec{v}}\right)$, are

$$
\left\{\begin{array}{l}
\mathrm{E}_{1}^{\vec{v}} \equiv\left(-\frac{\xi^{1}-v}{\sqrt{2 \theta}}+\frac{1}{\sqrt{30}} \frac{\left|\xi^{1}-v\right|^{2}+\left|\xi^{2}\right|^{2}+\left|\xi^{3}\right|^{2}}{\theta}\right) \mathrm{M}^{\frac{1}{2}} \\
\mathrm{E}_{2}^{\vec{v}} \equiv\left(-\sqrt{\frac{5}{2}}+\frac{1}{\sqrt{10}} \frac{\left|\xi^{1}-v\right|^{2}+\left|\xi^{2}\right|^{2}+\left|\xi^{3}\right|^{2}}{\theta}\right) \mathrm{M}^{\frac{1}{2}} \\
\mathrm{E}_{3}^{\vec{v}} \equiv\left(\frac{\xi^{1}-v}{\sqrt{2 \theta}}+\frac{1}{\sqrt{30}} \frac{\left|\xi^{1}-v\right|^{2}+\left|\xi^{2}\right|^{2}+\left|\xi^{3}\right|^{2}}{\theta}\right) \mathrm{M}^{\frac{1}{2}}
\end{array}\right.
$$

and these three vectors diagonalize the transport matrix $\mathrm{P}_{0}^{\vec{v}} \xi^{1}$ in $\operatorname{Range}\left(\mathrm{P}_{0}^{\vec{v}}\right)$ :

$$
\mathrm{P}_{0}^{\overrightarrow{\boldsymbol{v}}} \xi^{1} \mathrm{E}_{j}^{\overrightarrow{\boldsymbol{v}}}=\lambda_{j}(\overrightarrow{\boldsymbol{v}}) \mathrm{E}_{j}^{\overrightarrow{\boldsymbol{v}}}
$$

where

$$
\left\{\lambda_{1}(\overrightarrow{\boldsymbol{v}}), \lambda_{2}(\overrightarrow{\boldsymbol{v}}), \lambda_{3}(\overrightarrow{\boldsymbol{v}})\right\}=\left\{v-\sqrt{\frac{5 \theta}{3}}, v, v+\sqrt{\frac{5 \theta}{3}}\right\} .
$$


When the reference state $\overrightarrow{\boldsymbol{v}}$ is omitted, the macro-micro decomposition is referenced to the state $(\varrho, v, \theta)=(1,0,1)$ :

$$
\begin{aligned}
&\left\{\begin{array}{l}
\overrightarrow{\boldsymbol{v}} \equiv(1,0,1), \\
\mathrm{P}_{0} \mathrm{~g} \equiv \mathrm{P}_{0}^{\vec{v}} \mathrm{~g}, \mathrm{P}_{1} \mathrm{~g} \equiv \mathrm{P}_{1}^{\vec{v}} \mathrm{~g}, \\
\mathrm{E}_{i} \equiv \mathrm{E}_{i}^{\vec{v}}, i=1,2,3,
\end{array}\right. \\
& \mathrm{P}_{0} \xi^{1} \mathrm{E}_{j}=\bar{\lambda}_{j} \mathrm{E}_{j},\left\{\bar{\lambda}_{1}, \bar{\lambda}_{2}, \bar{\lambda}_{3}\right\}=\left\{-\sqrt{\frac{5}{3}}, 0, \sqrt{\frac{5}{3}}\right\} .
\end{aligned}
$$

Let $\left(\overrightarrow{\boldsymbol{u}}_{-}, \overrightarrow{\boldsymbol{u}}_{+}\right)$be a stationary 3 -shock wave of the compressible Euler equation (1.2) (in the form of (1.3)), and we denote the macro-micro decompositions with respect to the two end states of this 3 -shock wave by

$$
\begin{aligned}
& \mathrm{P}_{0}^{ \pm} \equiv \mathrm{P}_{0}^{\vec{u}_{ \pm}}, \mathrm{P}_{1}^{ \pm} \equiv \mathrm{P}_{1}^{\vec{u}_{ \pm}}, \\
& \mathrm{E}_{i}^{ \pm} \equiv \mathrm{E}_{i}^{\vec{u}_{ \pm}} \text {for } i=1,2,3 .
\end{aligned}
$$

Furthermore, we may rewrite the two operators $\mathrm{P}_{0}^{ \pm}$in the form

$$
\mathrm{P}_{0}^{ \pm}=\sum_{j=1}^{3} \mathrm{E}_{j}^{ \pm, *},
$$

where each component $\mathrm{E}_{j}^{ \pm, *}$ is a tensor product, $\mathrm{E}_{j}^{ \pm, *}=\mathrm{E}_{j}^{ \pm} \otimes\left\langle\mathrm{E}_{j}\right|$. The explicit form is

$$
\mathrm{E}_{l}^{ \pm, *} \mathrm{~g} \equiv \mathrm{E}_{j}^{ \pm} \otimes\left\langle\mathrm{E}_{j}\right| \mathrm{g} \equiv\left(\mathrm{E}_{l}^{ \pm}, \mathrm{g}\right) \mathrm{E}_{l}^{ \pm} \text {for } l=1,2,3 .
$$

2.4. Boltzmann shock layers. The Boltzmann shock profile $\varphi(x)$ is a travelling wave solution, $F(x, t, \xi)=\varphi(x-s t, \xi)$, of (1.1) connecting two Maxwellians given by the end states of a shock wave $\left(\overrightarrow{\boldsymbol{u}}_{-}, \overrightarrow{\boldsymbol{u}}_{+}\right)$, i.e.

$$
\left\{\begin{array}{l}
\left(\xi^{1}-s\right) \partial_{x} \varphi=Q(\varphi), \\
\lim _{x \rightarrow \pm \infty} \varphi(x)=\mathrm{M}_{\left[\rho_{ \pm}, u_{ \pm}, \boldsymbol{T}_{ \pm}\right]},
\end{array}\right.
$$

where $s$ is the shock speed of a given shock wave $\left(\overrightarrow{\boldsymbol{u}}_{-}, \overrightarrow{\boldsymbol{u}}_{+}\right)$of (1.2).

Theorem 2.7 (Caflisch-Nicolaenko). Suppose that the end states $\left(\rho_{ \pm}, u_{ \pm}, \boldsymbol{T}_{ \pm}\right)$of a shock wave are sufficiently close. Then there exists a one-parameter family of the Boltzmann shock profiles, $\varphi(x, \xi)$, connecting the Maxwellian states $\mathrm{M}_{\left[\rho_{ \pm}, u_{ \pm}, \boldsymbol{T}_{ \pm}\right]}$. Furthermore, the profile $\varphi(x, \xi)$ satisfies

$$
\left\|\varphi(x, \cdot)-\psi_{N S}(x, \xi)\right\|_{L_{\xi}^{2}} \leq O(1) \varepsilon^{2} e^{-\varepsilon|x| / D} \text { for some } D>0,
$$

where $\varepsilon=\left|\rho_{-}-\rho_{+}\right|+\left|u_{-}-u_{+}\right|+\left|\boldsymbol{T}_{-}-\boldsymbol{T}_{+}\right|, \psi_{N S}(x, \xi) \equiv \mathrm{M}_{[\bar{\rho}(x), \bar{u}(x), \overline{\boldsymbol{T}}(x)]}$, and the state $(\bar{\rho}(x), \bar{u}(x), \overline{\boldsymbol{T}}(x))$ is the shock profile of a compressible Navier-Stokes equation connecting the shock wave $\left(\overrightarrow{\boldsymbol{u}}_{-}, \overrightarrow{\boldsymbol{u}}_{+}\right)$:

$$
\left\{\begin{array}{l}
-s \partial_{x} \bar{\rho}+\partial_{x}(\bar{\rho} \bar{u})=0, \\
-s \partial_{x}(\bar{\rho} \bar{u})+\partial_{x}\left(\bar{\rho} \bar{u}^{2}+\bar{\rho} \overline{\boldsymbol{T}}\right)=\frac{4}{3} \eta \partial_{x}^{2} \bar{u} \\
-s \partial_{x}\left(\frac{\bar{\rho}\left(\bar{u}^{2}+3 \overline{\boldsymbol{T}}\right)}{2}\right)+\partial_{x}\left(\frac{\bar{\rho}\left(\bar{u}^{2}+5 \overline{\boldsymbol{T}}\right)}{2} \bar{u}\right)=\partial_{x}^{2}(\bar{\rho} \overline{\boldsymbol{T}})+\frac{4}{3} \eta \partial_{x}\left(\bar{u} \partial_{x} \bar{u}\right), \\
\lim _{x \rightarrow \pm \infty}(\bar{\rho}(x), \bar{u}(x), \overline{\boldsymbol{T}}(x))=\left(\rho_{ \pm}, u_{ \pm}, \boldsymbol{T}_{ \pm}\right) .
\end{array}\right.
$$


2.5. The Green's function of a linearized Boltzmann equation around a global Maxwellian. For the linearized Boltzmann equation around a global Maxwellian state $\mathrm{M}_{0} \equiv \mathrm{M}_{[1,0,1]}$,

$$
\partial_{t} \mathrm{~g}+\xi^{1} \partial_{x} \mathrm{~g}-\mathrm{Lg}=0
$$

its Green's function $\mathbb{G}\left(x, t, \xi, \xi_{*}\right)$ is a solution of a particular initial condition (completely particle-like initial data):

$$
\mathbb{G}\left(x, 0, \xi, \xi_{*}\right)=\delta(x) \delta^{3}\left(\xi-\xi_{*}\right) .
$$

Theorem 2.8 (Liu-Yu [13]). The Green's function consists of the particle-like waves propagating with a microscopic velocity and fluid-like waves propagating along Euler characteristics. More precisely, the Green's function satisfies for some positive constant $C_{1}$,

$$
\begin{aligned}
& \| \mathbb{G}\left(x, t, \xi, \xi_{*}\right)-e^{-\nu\left(\xi_{*}\right) t} \delta\left(x-\xi^{1} t\right) \delta^{3}\left(\xi-\xi_{*}\right)-\mathrm{j}^{1}\left(x, t, \xi, \xi_{*}\right)-\mathrm{j}^{2}\left(x, t, \xi, \xi_{*}\right) \\
& -\sum_{k=1}^{3} \frac{e^{-\frac{\left(x-\bar{\lambda}_{k} t\right)^{2}}{4 A_{k}(t+1)}}}{\sqrt{4 A_{k} \pi(t+1)}} \mathrm{E}_{k}(\xi) \mathrm{E}_{k}\left(\xi_{*}\right) \|_{L_{\xi}^{2}} \leq O(1)\left[\sum_{k=1}^{3} \frac{e^{-\frac{\left(x-\bar{\lambda}_{i} t\right)^{2}}{C_{0}(t+1)}}}{(t+1)}+e^{-(|x|+t) / C_{1}}\right] .
\end{aligned}
$$

where

$$
\begin{aligned}
\mathrm{j}^{1}\left(x, t, \xi, \xi_{*}\right)= & \left\{\begin{array}{l}
0 \text { if }\left(x-\xi_{*}^{1} t\right)\left(\xi^{1} t-x\right)<0, \\
\frac{e^{-\nu(\xi)\left(t-t_{1}\right)-\nu\left(\xi_{*}\right) t_{1}} K\left(\xi, \xi_{*}\right)}{\left(\xi^{1}-\xi_{*}^{1}\right)} \text { otherwise, }
\end{array}\right. \\
\text { where } t_{1}=t & -\frac{\left(x-\xi_{*}^{1} t\right)}{\xi^{1}-\xi_{*}^{1}}, \\
\mathrm{j}^{2}\left(x, t, \xi, \xi_{*}\right) \leq & O(1) e^{-\frac{\nu_{0}(t+|x|)}{3}}\left\{\begin{array}{l}
\left|\xi-\xi_{*}\right|^{-1}(1+|\xi|)^{-1} \text { for }\left|\xi-\xi_{*}\right| \leq 1, \\
(1+|\xi|)^{-1} \text { for }\left|\xi-\xi_{*}\right| \geq 1,
\end{array}\right.
\end{aligned}
$$

where $K\left(\xi, \xi_{*}\right)$ is given in (2.4) and $\left\{\bar{\lambda}_{1}, \bar{\lambda}_{2}, \bar{\lambda}_{3}\right\}=\left\{-\sqrt{\frac{5}{3}}, 0, \sqrt{\frac{5}{3}}\right\}$ are given in (2.8). Here $C_{0}$ is any constant satisfying

$$
C_{0}>4 \max \left(A_{1}, A_{2}, A_{3}\right)
$$

and $A_{i}$ is defined by $A_{i} \equiv-\left(\mathrm{P}_{1} \xi^{1} \mathrm{E}_{j}, \mathrm{~L}^{-1} \mathrm{P}_{1} \xi^{1} \mathrm{E}_{j}\right)$.

The construction to obtain the pointwise structure of the Green's function $\mathbb{G}\left(x, t, \xi, \xi_{*}\right)$ for (2.15) can be applied to obtain the pointwise structure of the Green's function $\mathbb{G}^{D, \varepsilon_{0}}\left(x, t, \xi, \xi_{*}\right)$ for the damped Boltzmann equation in the $\mathrm{E}_{1}$ and $\mathrm{E}_{2}$ directions with $\varepsilon_{0}>0$ :

$$
\partial_{t} \mathrm{~g}+\xi^{1} \partial_{x} \mathrm{~g}-\mathrm{Lg}+\varepsilon_{0} \sum_{j=1}^{2}\left(\mathrm{E}_{j}, \mathrm{~g}\right) \mathrm{E}_{j}=0 .
$$

\section{Corollary 2.9.}

$$
\begin{gathered}
\| \mathbb{G}^{D, \varepsilon_{0}}\left(x, t, \xi, \xi_{*}\right)-e^{-\nu\left(\xi_{*}\right) t} \delta\left(x-\xi^{1} t\right) \delta^{3}\left(\xi-\xi_{*}\right)-\mathrm{j}^{D, \varepsilon_{0} ; 1}\left(x, t, \xi, \xi_{*}\right)-\mathrm{j}^{D, \varepsilon_{0} ; 2}\left(x, t, \xi, \xi_{*}\right) \\
-\sum_{k=1}^{2} \frac{e^{-\frac{\left(x-\bar{\lambda}_{k} t\right)^{2}}{4 A_{k}(t+1)}}}{\sqrt{4 A_{k} \pi(t+1)}} \mathrm{E}_{k}(\xi) \mathrm{E}_{k}\left(\xi_{*}\right)-\frac{e^{-\frac{\left(x-\bar{\lambda}_{3}\right)^{2}}{4 A_{3}(t+1)}-\varepsilon_{0} t}}{\sqrt{4 A_{3} \pi(t+1)}} \mathrm{E}_{3}(\xi) \mathrm{E}_{3}\left(\xi_{*}\right) \|_{L_{\xi}^{2}} \\
\leq O(1)\left[\sum_{k=1}^{2} \frac{e^{-\frac{\left(x-\bar{\lambda}_{i} t\right)^{2}}{C_{0}(t+1)}}}{(t+1)}+\frac{e^{-\frac{\left(x-\bar{\lambda}_{3}\right)^{2}}{C_{0}(t+1)}-\varepsilon_{0} t}}{(t+1)}+e^{-(|x|+t) / C_{1}}\right]
\end{gathered}
$$


where

$$
\begin{aligned}
& \mathrm{j}^{D, \varepsilon ; 1}\left(x, t, \xi, \xi_{*}\right)=\left\{\begin{array}{l}
0 \text { if }\left(x-\xi_{*}^{1} t\right)\left(\xi^{1} t-x\right)<0, \\
\frac{e^{-\nu(\xi)\left(t-t_{1}\right)-\nu\left(\xi_{*}\right) t_{1}} K\left(\xi, \xi_{*}\right)}{\left(\xi^{1}-\xi_{*}^{1}\right)} \text { otherwise, }
\end{array}\right. \\
& \text { where } t_{1}=t-\frac{\left(x-\xi_{*}^{1} t\right)}{\xi^{1}-\xi_{*}^{1}}, \\
& \mathrm{j}^{D, \varepsilon_{0} ; 2}\left(x, t, \xi, \xi_{*}\right) \leq O(1) e^{-\frac{\nu_{0}(t+|x|)}{3}}\left\{\begin{array}{l}
\left|\xi-\xi_{*}\right|^{-1}(1+|\xi|)^{-1} \text { for }\left|\xi-\xi_{*}\right| \leq 1, \\
(1+|\xi|)^{-1} \text { for }\left|\xi-\xi_{*}\right| \geq 1 .
\end{array}\right.
\end{aligned}
$$

Remark 2.10. The damping mechanism on the $\mathrm{E}_{1}$ and $\mathrm{E}_{2}$ components is designed for the 3 -shock wave $\left(\overrightarrow{\boldsymbol{u}}_{-}, \overrightarrow{\boldsymbol{u}}_{+}\right)$. For a 1 -shock wave case, the damping terms will be added on the $E_{2}$ and $E_{3}$ components, instead.

One can view the Green's function $\mathbb{G}\left(x, t, \xi, \xi_{*}\right)$ of (2.15) as an $L_{\xi}^{2}$-operatorvalued function $\mathbb{G}(x, t)$. With the analysis of the long-wave-short-wave decomposition in Section 3.2 and 3.3 of [13, one can have the following corollary for $\mathbb{G}(x, t)$ and $\mathbb{G}^{D, \varepsilon_{0}}(x, t)$ :

Corollary 2.11. For each given $j \in\{1,2,3\}$ the Green's functions $\mathbb{G}(x, t)$ and $\mathbb{G}^{D, \varepsilon_{0}}(x, t)$ satisfy for $\left|x-\bar{\lambda}_{j} t\right| \leq \frac{1}{2} \sqrt{\frac{5}{3}}(1+t)$ and for $t>1$

$$
\left\{\begin{array}{l}
\left\|\mathrm{E}_{j}^{*} \mathbb{G}(x, t) \mathrm{E}_{j}^{*}\right\|_{L_{\xi}^{2}} \leq O(1) \frac{e^{-\frac{\left(x-\bar{\lambda}_{j} t\right)^{2}}{C_{0}(1+t)}}}{\sqrt{(1+t)}} \\
\left\|\mathrm{E}_{j}^{*} \mathbb{G}^{D, \varepsilon_{0}}(x, t) \mathrm{E}_{j}^{*}\right\|_{L_{\xi}^{2}} \leq O(1) \frac{e^{-\frac{\left(x-\bar{\lambda}_{j} t\right)^{2}}{C_{0}(1+t)}-\left(1-\delta_{3}^{j}\right)} \frac{\varepsilon_{0} t}{C_{0}}}{\sqrt{(1+t)}}
\end{array}\right.
$$

for $k \neq j$

$$
\left\{\begin{array}{l}
\left\|\mathrm{E}_{j}^{*} \mathbb{G}(x, t) \mathrm{E}_{k}^{*}\right\|_{L_{\xi}^{2}},\left\|\mathrm{E}_{k}^{*} \mathbb{G}(x, t) \mathrm{E}_{j}^{*}\right\|_{L_{\xi}^{2}} \leq O(1) \frac{e^{-\frac{\left(x-\bar{\lambda}_{j} t\right)^{2}}{C_{0}(1+t)}}}{(1+t)} \\
\left\|\mathrm{E}_{j}^{*} \mathbb{G}^{D, \varepsilon_{0}}(x, t) \mathrm{E}_{k}^{*}\right\|_{L_{\xi}^{2}},\left\|\mathrm{E}_{k}^{*} \mathbb{G}^{D, \varepsilon_{0}}(x, t) \mathrm{E}_{j}^{*}\right\|_{L_{\xi}^{2}} \leq O(1) \frac{e^{-\frac{\left(x-\bar{\lambda}_{j} t\right)^{2}}{C_{0}(1+t)}-\left(1-\delta_{3}^{j}\right)} \frac{\varepsilon_{0} t}{C_{0}}}{(1+t)}
\end{array}\right.
$$

$$
\left\{\begin{array}{l}
\left\|\mathrm{E}_{j}^{*} \mathbb{G}(x, t) \mathrm{P}_{1}\right\|_{L_{\xi}^{2}},\left\|\mathrm{P}_{1} \mathbb{G}(x, t) \mathrm{E}_{j}^{*}\right\|_{L_{\xi}^{2}} \leq O(1) \frac{e^{-\frac{\left(x-\bar{\lambda}_{j} t\right)^{2}}{C_{0}(1+t)}}}{(1+t)} \\
\left\|\mathrm{E}_{j}^{*} \mathbb{G}^{D, \varepsilon_{0}}(x, t) \mathrm{P}_{1}\right\|_{L_{\xi}^{2}},\left\|\mathrm{P}_{1} \mathbb{G}^{D, \varepsilon_{0}}(x, t) \mathrm{E}_{j}^{*}\right\|_{L_{\xi}^{2}} \leq O(1) \frac{e^{-\frac{\left(x-\bar{\lambda}_{j} t\right)^{2}}{C_{0}(1+t)}-\left(1-\delta_{3}^{j}\right)} \frac{\varepsilon_{0} t}{C_{0}}}{(1+t)}
\end{array}\right.
$$

$$
\left\{\begin{array}{l}
\left\|\mathrm{P}_{1} \mathbb{G}(x, t) \mathrm{E}_{k}^{*}\right\|_{L_{\xi}^{2}},\left\|\mathrm{E}_{k}^{*} \mathbb{G}(x, t) \mathrm{P}_{1}\right\|_{L_{\xi}^{2}} \leq O(1) \frac{e^{-\frac{\left(x-\bar{\lambda}_{j} t\right)^{2}}{C_{0}(1+t)}}}{(1+t)^{3 / 2}} \\
\left\|\mathrm{P}_{1} \mathbb{G}^{D, \varepsilon_{0}}(x, t) \mathrm{E}_{k}^{*}\right\|_{L_{\xi}^{2}},\left\|\mathrm{E}_{k}^{*} \mathbb{G}(x, t) \mathrm{P}_{1}\right\|_{L_{\xi}^{2}} \leq O(1) \frac{e^{-\frac{\left(x-\bar{\lambda}_{j} t\right)^{2}}{C_{0}(1+t)}-\left(1-\delta_{3}^{j}\right) \frac{\varepsilon_{0} t}{C_{0}}}}{(1+t)^{3 / 2}}
\end{array}\right.
$$

for $k, l \neq j$,

$$
\left\{\begin{array}{l}
\left\|\mathrm{E}_{l}^{*} \mathbb{G}(x, t) \mathrm{E}_{k}^{*}\right\|_{L_{\xi}^{2}},\left\|\mathrm{E}_{k}^{*} \mathbb{G}(x, t) \mathrm{E}_{l}^{*}\right\|_{L_{\xi}^{2}} \leq O(1) \frac{e^{-\frac{\left(x-\bar{\lambda}_{j} t\right)^{2}}{C_{0}(1+t)}}}{(1+t)^{3 / 2}} \\
\left\|\mathrm{E}_{l}^{*} \mathbb{G}^{D, \varepsilon_{0}}(x, t) \mathrm{E}_{k}^{*}\right\|_{L_{\xi}^{2}},\left\|\mathrm{E}_{k}^{*} \mathbb{G}^{D, \varepsilon_{0}}(x, t) \mathrm{E}_{l}^{*}\right\|_{L_{\xi}^{2}} \leq O(1) \frac{e^{-\frac{\left(x-\bar{\lambda}_{j} t\right)^{2}}{C_{0}(1+t)}-\left(1-\delta_{3}^{j}\right)} \frac{\varepsilon_{0} t}{C_{0}}}{(1+t)^{3 / 2}},
\end{array}\right.
$$

where the operator $\mathrm{E}_{k}^{*}$ is defined in (2.11). 


\section{Comparisons on the Green's functions AROUND THE END STATES OF A SHOCK WAVE}

In the rest of this paper, the shock wave $\left(\overrightarrow{\boldsymbol{u}}_{-}, \overrightarrow{\boldsymbol{u}}_{+}\right)$is assumed to be a stationary 3shock wave of the compressible Euler equation with a sufficient weak shock strength

$$
\varepsilon \equiv\left|\rho_{-}-\rho_{+}\right|+\left|u_{-}-u_{+}\right|+\left|\boldsymbol{T}_{-}-\boldsymbol{T}_{+}\right| \ll 1 .
$$

Let $\mathrm{M}_{ \pm}$be the Maxwellian states related to the end states of the 3-shock wave $\left(\overrightarrow{\boldsymbol{u}}_{-}, \overrightarrow{\boldsymbol{u}}_{+}\right)$:

$$
\mathrm{M}_{-} \equiv \mathrm{M}_{\left[\rho_{-}, u_{-}, \boldsymbol{T}_{-}\right]} \text {and } \mathrm{M}_{+} \equiv \mathrm{M}_{\left[\rho_{+}, u_{+}, \boldsymbol{T}_{+}\right]} .
$$

We compare the solutions of the following two equations:

$$
\begin{aligned}
& \partial_{t} \mathrm{~g}_{-}+\xi^{1} \partial_{x} \mathrm{~g}_{-}-\mathrm{L}_{-} \mathrm{g}_{-}=0, \\
& \partial_{t} \mathrm{~g}_{+}+\xi^{1} \partial_{x} \mathrm{~g}_{+}-\mathrm{L}_{+} \mathrm{g}_{+}=0,
\end{aligned}
$$

where $L_{ \pm}$are the linearized collision operators around $M_{ \pm}$with $\sqrt{M_{-}}$as the fixed reference Maxwellian state for both operators $L_{ \pm}$:

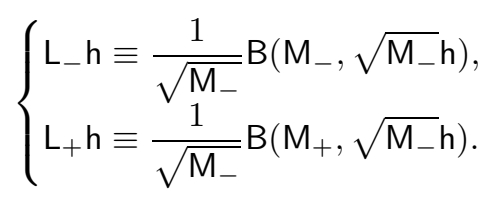

The Green's functions of (3.1) and (3.2) are denoted, respectively, by $\mathbb{G}_{-}\left(x, t, \xi, \xi_{*}\right)$ and $\mathbb{G}_{+}\left(x, t, \xi, \xi_{*}\right)$.

Since $L_{-}$uses $\sqrt{M_{-}}$as the reference Maxwellian state, one can relate the Green's functions $\mathbb{G}_{-}$and $\mathbb{G}$, which use $\sqrt{\mathrm{M}_{0}}$ as the reference state, together, by changing the coordinate system,

$$
\left\{\begin{array}{l}
\bar{x}=\frac{x-u_{-} t}{\sqrt{\boldsymbol{T}_{-}}}, \\
\bar{t}=t \\
\bar{\xi}=\left(\xi^{1}-u_{-}, \xi^{2}, \xi^{3}\right) / \sqrt{\boldsymbol{T}_{-}} \\
\bar{\xi}_{*}=\left(\xi_{*}^{1}-u_{-}, \xi^{2}, \xi^{3}\right) / \sqrt{\boldsymbol{T}_{-}} .
\end{array}\right.
$$

M- $(\xi)$ becomes $\boldsymbol{T}_{-}^{-3 / 2} \mathrm{M}_{0}(\bar{\xi})$, and the Boltzmann equation (3.1) becomes (2.15). It follows that

$$
\mathbb{G}_{-}\left(x, t, \xi, \xi_{*}\right)=\frac{1}{\boldsymbol{T}_{-}^{2}} \mathbb{G}\left(\frac{x-u_{-} t}{\sqrt{\boldsymbol{T}_{-}}}, t, \bar{\xi}, \bar{\xi}_{*}\right) .
$$

We apply the subscripts \pm to the Green's functions of the damped equations below and denote them by $\mathbb{G}_{ \pm}^{D, \varepsilon_{0}}\left(x, t, \xi, \xi_{*}\right)$ :

$$
\partial_{t} \mathrm{~h}_{ \pm}+\xi^{1} \partial_{x} \mathrm{~h}_{ \pm}-\mathrm{L}_{ \pm} \mathrm{h}_{ \pm}+\varepsilon_{0} \sum_{j=1}^{2}\left(\mathrm{l}_{j}, \mathrm{~h}_{ \pm}\right) \mathrm{r}_{j}=0 .
$$

Then by the rescaling (3.4), Corollary 2.11 can be rewritten as follows. 
Corollary 3.1. There exists $D_{0}>0$ such that the Green's functions $\mathbb{G}_{-}(x, t)$ and $\mathbb{G}_{-}^{D, \varepsilon_{0}}(x, t)$ satisfy for $\left|x-\lambda_{j}^{-} t\right| \leq \frac{\min \left(\boldsymbol{c}_{-}, \boldsymbol{c}_{+}\right)}{2}(1+t)$ and for $t>1$,

$$
\left\{\begin{array}{l}
\left\|\mathrm{E}_{j}^{-, *} \mathbb{G}_{-}(x, t) \mathrm{E}_{j}^{-, *}\right\|_{L_{\xi}^{2}} \leq O(1) \frac{e^{-\frac{(x-\lambda-t)^{2}}{D_{0}(1+t)}}}{\sqrt{(1+t)}}, \\
\left\|\mathrm{E}_{j}^{-, *} \mathbb{G}_{-}^{D, \varepsilon_{0}}(x, t) \mathrm{E}_{j}^{-, *}\right\|_{L_{\xi}^{2}} \leq O(1) \frac{e^{-\frac{\left(x-\lambda_{j}^{-} t\right)^{2}}{D_{0}(1+t)}-\left(1-\delta_{3}^{j}\right) \frac{\varepsilon_{0} t}{D_{0}}}}{\sqrt{(1+t)}},
\end{array}\right.
$$

for $k \neq j$

$$
\left\{\begin{array}{l}
\left\|\mathrm{E}_{j}^{-, *} \mathbb{G}_{-}(x, t) \mathrm{E}_{k}^{-, *}\right\|_{L_{\xi}^{2}},\left\|\mathrm{E}_{k}^{-, *} \mathbb{G}_{-}(x, t) \mathrm{E}_{j}^{-, *}\right\|_{L_{\xi}^{2}} \leq O(1) \frac{e^{-\frac{\left(x-\lambda_{j}^{-} t\right)^{2}}{D_{0}(1+t)}}}{(1+t)}, \\
\left\|\mathrm{E}_{j}^{-, *} \mathbb{G}_{-}^{D, \varepsilon_{0}}(x, t) \mathrm{E}_{k}^{-, *}\right\|_{L_{\xi}^{2}},\left\|\mathrm{E}_{k}^{-, *} \mathbb{G}_{-}^{D, \varepsilon_{0}}(x, t) \mathrm{E}_{j}^{-, *}\right\|_{L_{\xi}^{2}} \leq O(1) \frac{e^{-\frac{\left(x-\lambda_{j}^{-} t\right)^{2}}{D_{0}(1+t)}-\left(1-\delta_{3}^{j}\right) \frac{\varepsilon_{0} t}{D_{0}}}}{(1+t)},
\end{array}\right.
$$

$$
\left\{\begin{array}{l}
\left\|\mathrm{E}_{j}^{-, *} \mathbb{G}_{-}(x, t) \mathrm{P}_{1}^{-}\right\|_{L_{\xi}^{2}},\left\|\mathrm{P}_{1}^{-} \mathbb{G}_{-}(x, t) \mathrm{E}_{j}^{-, *}\right\|_{L_{\xi}^{2}} \leq O(1) \frac{e^{-\frac{(x-\lambda \bar{j}}{D_{0}(1+t)}}}{(1+t)}, \\
\left\|\mathrm{E}_{j}^{-, * *} \mathbb{G}_{-}^{D, \varepsilon_{0}}(x, t) \mathrm{P}_{1}^{-}\right\|_{L_{\xi}^{2}},\left\|\mathrm{P}_{1}^{-} \mathbb{G}_{-}^{D, \varepsilon_{0}}(x, t) \mathrm{E}_{j}^{-, *}\right\|_{L_{\xi}^{2}} \leq O(1) \frac{e^{-\frac{(x-\lambda-\bar{j}}{D_{0}(1+t)}-\left(1-\delta_{3}^{j}\right) \frac{\varepsilon_{0} t}{D_{0}}}}{(1+t)},
\end{array}\right.
$$

for $k, l \neq j$

$$
\left\{\begin{array}{l}
\left\|\mathrm{E}_{l}^{-, *} \mathbb{G}_{-}(x, t) \mathrm{E}_{k}^{-, *}\right\|_{L_{\xi}^{2}},\left\|\mathrm{E}_{k}^{-, *} \mathbb{G}_{-}(x, t) \mathrm{E}_{l}^{-, *}\right\|_{L_{\xi}^{2}} \leq O(1) \frac{e^{-\frac{\left(x-\lambda_{j}^{-} t\right)^{2}}{D_{0}(1+t)}}}{(1+t)^{3 / 2}}, \\
\left\|\mathrm{E}_{l}^{-, *} \mathbb{G}_{-}^{D, \varepsilon_{0}}(x, t) \mathrm{E}_{k}^{-, *}\right\|_{L_{\xi}^{2}},\left\|\mathrm{E}_{k}^{-, * *} \mathbb{G}_{-}^{D, \varepsilon_{0}}(x, t) \mathrm{E}_{l}^{-, *}\right\|_{L_{\xi}^{2}} \leq O(1) \frac{e^{-\frac{\left(x-\lambda_{j}^{-} t\right)^{2}}{D_{0}(1+t)}-\left(1-\delta_{3}^{j}\right) \frac{\varepsilon_{0} t}{D_{0}}}}{(1+t)^{3 / 2}},
\end{array}\right.
$$

for $k \neq j$

$$
\left\{\begin{array}{l}
\left\|\mathrm{P}_{1}^{-} \mathbb{G}_{-}(x, t) \mathrm{E}_{k}^{-, *}\right\|_{L_{\xi}^{2}},\left\|\mathrm{E}_{k}^{-, *} \mathbb{G}_{-}(x, t) \mathrm{P}_{1}^{-}\right\|_{L_{\xi}^{2}} \leq O(1) \frac{e^{-\frac{\left(x-\lambda_{j}^{-} t\right)^{2}}{D_{0}(1+t)}}}{\left(1+t^{3 / 2}\right.}, \\
\left\|\mathrm{P}_{1}^{-} \mathbb{G}_{-}^{D, \varepsilon_{0}}(x, t) \mathrm{E}_{k}^{-, *}\right\|_{L_{\xi}^{2}},\left\|\mathrm{E}_{k}^{-, *} \mathbb{G}_{-}(x, t) \mathrm{P}_{1}^{-}\right\|_{L_{\xi}^{2}} \leq O(1) \frac{e^{-\frac{(x-\lambda j}{D_{0}(1+t)}-\left(1-\delta_{3}^{j}\right) \frac{\varepsilon_{0} t}{D_{0}}}}{(1+t)^{3 / 2}} .
\end{array}\right.
$$

Here the operator $\mathrm{E}_{k}^{-, *}$ is defined in (2.11) and

$$
\left\{\lambda_{1}^{ \pm}, \lambda_{2}^{ \pm}, \lambda_{3}^{ \pm}\right\} \equiv\left\{u_{ \pm}-\sqrt{\frac{5 \boldsymbol{T}_{ \pm}}{3}}, u_{ \pm}, u_{ \pm}+\sqrt{\frac{5 \boldsymbol{T}_{ \pm}}{3}}\right\},
$$

and $\boldsymbol{c}_{ \pm}=\sqrt{\frac{5 \boldsymbol{T}_{ \pm}}{3}}$ are the sound speeds at rest with temperatures $\boldsymbol{T}_{ \pm}$, and the parameter $D_{0}$ is chosen to satisfy

$$
D_{0}>\max \left(C_{0}, C_{1}\right)
$$

with $C_{0}$ and $C_{1}$ in Theorem 2.8 and

$$
\varepsilon^{-2} \sup _{x \in \mathbb{R}}\left\|\varphi^{\prime}(x)\right\|_{L_{\xi}^{2}} e^{\frac{4 \varepsilon|x|}{D_{0}}}=O(1) .
$$

Remark 3.2. The rescaling property in (3.4) cannot be generalized to $\mathbb{G}_{+}(x, t, \xi, \xi)$ due to the asymmetry of $M_{-}$in $M_{-}^{-\frac{1}{2}} B\left(M_{+}, M_{-}^{\frac{1}{2}} g\right)$. However, the discrete spectrum of the operator $-i \xi^{1} \eta+\mathrm{L}_{+}$for $|\eta| \ll 1$ is identical to the spectrum of the operator 
$-i \xi^{1} \eta+\overline{\mathrm{L}}_{+}$, since both operators are conjugates so that the spectrum information $\left(\psi_{+}(\eta), \sigma_{+}(\xi)\right)$ of $\left(-i \xi^{1} \eta+\overline{\mathrm{L}}_{+}\right) \psi_{+, j}(\eta)=\sigma_{+, j}(\eta) \psi_{+, j}(\eta), j=1,2,3$, in [13] can be applied, where $\overline{\mathrm{L}}_{+} \mathrm{g} \equiv 2 \mathrm{M}_{+}^{-1 / 2} \mathrm{~B}\left(\mathrm{M}_{+}, \mathrm{M}_{+}^{1 / 2} \mathrm{~g}\right)$. By using this spectrum information, one can get the long-wave component $\mathbb{G}_{+L}(x, t)$ of $\mathbb{G}_{+}(x, t)$ in the sense of an $L_{\xi^{-}}^{2}$ operator-valued function:

$$
\mathbb{G}_{+L}(x, t) \equiv \sum_{j=1}^{3} \int_{|\eta|<\kappa_{0}} e^{i \eta x+\sigma_{+, j}(\eta) t}\left(\left(\frac{\mathrm{M}_{-}}{\mathrm{M}_{+}}\right)^{\frac{1}{2}} \psi_{+, j}(\eta)\right) \otimes\left\langle\left(\frac{\mathrm{M}_{+}}{\mathrm{M}_{-}}\right)^{\frac{1}{2}} \psi_{+, j}(\eta)\right| d \eta .
$$

Then one just follows the same proof in 13 for the parts in the short wave component, particle-wave decomposition, energy estimates for waves outside high Mach regimes. Then one can obtain that

$$
\left\|\mathbb{G}_{+}(x, t)\right\|_{L_{\xi}^{2}} \leq O(1)\left(\sum_{j=1}^{3} \frac{e^{-\frac{\left(x-\lambda_{j}^{+} t\right)^{2}}{D_{0}(1+t)}}}{\sqrt{(1+t)}}+e^{-(|x|+t) / D_{0}}\right) .
$$

Furthermore, following the construction in [13, one has the following corollary:

Corollary 3.3. For any $|x| \leq 2 \boldsymbol{c}(1+t), \boldsymbol{c} \equiv \sqrt{\frac{5 \boldsymbol{T}_{-}}{3}}, t>1$,

$$
\begin{aligned}
& \left\|\mathrm{E}_{k}^{-, * *} \mathbb{G}_{+}(x, t) \mathrm{E}_{l}^{-, *}\right\|_{L_{\xi}^{2}} \\
& \quad \leq O(1) \sum_{j=1}^{3}\left[(1+t)^{-\frac{2-\delta_{k}^{j}-\delta_{l}^{j}}{2}}+\varepsilon^{1-\delta_{k}^{j}}(1+t)^{-\frac{1-\delta_{l}^{j}}{2}}\right] \frac{e^{-\frac{\left(x-\lambda_{j}^{+} t\right)^{2}}{D_{0}(1+t)}}}{\sqrt{(1+t)}} \\
& \quad+O(1) \sum_{j=1}^{3}\left[(1+t)^{-\frac{1-\delta_{l}^{j}}{2}}+\varepsilon^{1-\delta_{l}^{j}}(1+t)^{-\frac{1-\delta_{k}^{j}}{2}}+\varepsilon^{2-\delta_{k}^{j}-\delta_{l}^{j}}\right] \frac{e^{-\frac{\left(x-\lambda_{j}^{+} t\right)^{2}}{D_{0}(1+t)}}}{\sqrt{(1+t)}}
\end{aligned}
$$

$$
\begin{aligned}
& \left\|\mathrm{P}_{1}^{-} \mathbb{G}_{+}(x, t) \mathrm{E}_{l}^{-, *}\right\|_{L_{\xi}^{2}} \\
& \quad \leq O(1) \sum_{j=1}^{3}\left[(1+t)^{-\frac{2-\delta_{l}^{j}}{2}}+\varepsilon(1+t)^{-\frac{1-\delta_{l}^{j}}{2}}+\varepsilon^{1-\delta_{l}^{j}}(1+t)^{-\frac{1}{2}}+\varepsilon^{2-\delta_{l}^{j}}\right] \frac{e^{-\frac{\left(x-\lambda_{j}^{+} t\right)^{2}}{D_{0}(1+t)}}}{\sqrt{(1+t)}},
\end{aligned}
$$

$$
\begin{aligned}
& \left\|\mathrm{E}_{l}^{-, *} \mathbb{G}_{+}(x, t) \mathrm{P}_{1}^{-}\right\|_{L_{\xi}^{2}} \\
& \quad \leq O(1) \sum_{j=1}^{3}\left[(1+t)^{-\frac{2-\delta_{l}^{j}}{2}}+\varepsilon(1+t)^{-\frac{1-\delta_{l}^{j}}{2}}+\varepsilon^{1-\delta_{l}^{j}}(1+t)^{-\frac{1}{2}}+\varepsilon^{2-\delta_{l}^{j}}\right] \frac{e^{-\frac{\left(x-\lambda_{j}^{+} t\right)^{2}}{D_{0}(1+t)}}}{\sqrt{(1+t)}} .
\end{aligned}
$$

Lemma 3.4. There exists $D_{0}>0$ such that the difference of the Green's functions $\mathbb{G}_{-}-\mathbb{G}_{+}$satisfies for $t>1$, 


$$
\begin{aligned}
\left\|\mathrm{E}_{k}^{-, *}\left(\mathbb{G}_{-}(x, t)-\mathbb{G}_{+}(x, t)\right) \mathrm{E}_{l}^{-, *}\right\|_{L_{\xi}^{2}} \leq O(1) e^{-(|x|+t) / D_{0}} \\
+O(1) \sum_{j=1}^{3} \frac{\left(e^{-\frac{\left(x-\lambda_{j}^{-} t\right)^{2}}{D_{0}(1+t)}}+e^{-\frac{\left(x-\lambda_{j}^{+} t\right)^{2}}{D_{0}(1+t)}}\right) \min (1, \varepsilon \sqrt{1+t})}{\sqrt{1+t}} \\
\\
\quad\left(\frac{1}{(1+t)^{\frac{\left(2-\delta_{k}^{j}-\delta_{l}^{j}\right)}{2}}}+\frac{\varepsilon^{1-\delta_{l}^{j}}}{(1+t)^{\frac{\left(1-\delta_{k}^{j}\right)}{2}}}+\frac{\varepsilon^{1-\delta_{k}^{j}}}{(1+t)^{\frac{\left(1-\delta_{l}^{j}\right)}{2}}}+\varepsilon^{2-\delta_{k}^{j}-\delta_{l}^{j}}\right),
\end{aligned}
$$

and

$$
\begin{aligned}
& \left\|\mathrm{P}_{1}^{-}\left(\mathbb{G}_{-}(x, t)-\mathbb{G}_{+}(x, t)\right) \mathrm{E}_{l}^{-, *}\right\|_{L_{\xi}^{2}} \\
& \leq O(1) \sum_{j=1}^{3} \frac{\left(e^{-\frac{\left(x-\lambda_{j}^{-} t\right)^{2}}{D_{0}(1+t)}}+e^{-\frac{\left(x-\lambda_{j}^{+} t\right)^{2}}{D_{0}(1+t)}}\right) \min (1, \varepsilon \sqrt{1+t})}{1+t} \\
& \cdot\left(\frac{1}{(1+t)^{\frac{\left(1-\delta_{l}^{j}\right)}{2}}}+\varepsilon^{1-\delta_{l}^{j}}\right)+O(1) e^{-(|x|+t) / D_{0}} .
\end{aligned}
$$

Proof. This lemma is a comparison of the spectral representations of the long wave components of

$$
\mathbb{G}_{-, L}(x, t)=\sum_{j=1}^{3} \int_{|\eta| \leq \kappa_{0}} e^{i \eta x+\sigma_{-, j}(\eta) t} \psi_{-, j}(\eta) \otimes\left\langle\psi_{j,-}(\eta)\right| d \eta
$$

and $\mathbb{G}_{+, L}$ given in (3.10) for some $\kappa_{0}>0$. Thus,

$$
\begin{aligned}
&(3.17) \\
&= \sum_{j=1}^{3} \int_{|\eta| \leq \kappa_{0}} e^{i \eta x}\left(e^{\sigma_{-, j}(\eta) t}-e^{\sigma_{+, j}(\eta) t}\right) \psi_{-, j}(\eta) \otimes\left\langle\psi_{j,-}(\eta)\right| d \eta \\
&+\sum_{j=1}^{3} \int_{|\eta| \leq \kappa_{0}} e^{i \eta x+\sigma_{+, j}(\eta) t}\left(\psi_{-, j}(\eta)-\left(\frac{\mathrm{M}_{-}}{\mathrm{M}_{+}}\right)^{\frac{1}{2}} \psi_{+, j}(\eta)\right) \otimes\left\langle\psi_{j,-}(\eta)\right| d \eta \\
&+\sum_{j=1}^{3} \int_{|\eta| \leq \kappa_{0}} e^{i \eta x+\sigma_{+, j}(\eta) t}\left(\left(\frac{\mathrm{M}_{-}}{\mathrm{M}_{+}}\right)^{\frac{1}{2}} \psi_{+, j}(\eta)\right) \otimes\left\langle\psi_{-, j}(\eta)-\left(\frac{\mathrm{M}_{+}}{\mathrm{M}_{-}}\right)^{\frac{1}{2}} \psi_{+, j}(\eta)\right| d \eta .
\end{aligned}
$$

This yields that for $|x| \leq 2 \boldsymbol{c}_{0}(1+t)$

$$
\int_{|\eta| \leq \kappa_{0}} e^{i x \eta}\left(e^{\sigma_{-, j}(\eta) t}-e^{\sigma_{+, j}(\eta) t}\right) d \eta \leq O(1) \min (1, \varepsilon \sqrt{t}) \frac{e^{-\frac{\left(x-\lambda_{j}^{-} t\right)^{2}}{D_{0}(1+t)}}+e^{-\frac{\left(x-\lambda_{j}^{+} t\right)^{2}}{D_{0}(1+t)}}}{\sqrt{(1+t)}} .
$$

Here this estimate is obtained in two cases: $t \leq \varepsilon^{-2}$ and $t \geq \varepsilon^{-2}$. From (3.17), 
(3.18), $\mathrm{E}_{k}^{-, *} \psi_{-, j}(\eta)=\delta_{j}^{k}+O(1) \eta$, and

$$
\mathrm{E}_{k}^{-, *}\left(\psi_{-, j}(\eta)-\left(\frac{\mathrm{M}_{-}}{\mathrm{M}_{+}}\right)^{\frac{1}{2}} \psi_{+, j}(\eta)\right)=O(1) \varepsilon
$$

together, this lemma follows.

With Lemma 3.4 one has the local structure around the shock front:

Lemma 3.5. For $k, l \neq 3,|x| \leq 2 c(1+t), t>1$, there exist $D_{1}>4 D_{0}$ and $E_{0}>0$ such that

$$
\begin{aligned}
& e^{-\frac{4 \varepsilon|x|}{D_{1}}}\left\|\mathrm{E}_{k}^{-, *}\left(\mathbb{G}_{+L}(x, t)-\mathbb{G}_{-L}(x, t)\right) \mathrm{E}_{l}^{-, *}\right\|_{L_{\xi}^{2}} \\
& \quad=O(1) \min (1, \varepsilon \sqrt{t})\left(\frac{1}{(1+t)^{3 / 2}}+\frac{\varepsilon^{2}}{(1+t)^{1 / 2}}\right) e^{-\frac{3 \varepsilon|x|}{D_{1}}-\frac{\varepsilon^{2} t}{E_{0}}} \\
& \quad+O(1) \frac{\min (1, \varepsilon \sqrt{t})}{(1+t)^{1 / 2}} e^{-\frac{3 \varepsilon|x|}{D_{1}}-\frac{\varepsilon t}{E_{0}}}, \\
& e^{-\frac{4 \varepsilon|x|}{D_{1}}}\left\|\mathrm{E}_{3}^{-, *}\left(\mathbb{G}_{+L}(x, t)-\mathbb{G}_{-L}(x, t)\right) \mathrm{E}_{l}^{-, *}\right\|_{L_{\xi}^{2}} \\
& \quad=O(1) \min (1, \varepsilon \sqrt{t})\left(\frac{1}{(1+t)}+\frac{\varepsilon}{(1+t)^{\frac{1}{2}}}\right) e^{-\frac{3 \varepsilon|x|}{D_{1}}-\frac{\varepsilon^{2} t}{E_{0}}} .
\end{aligned}
$$

Proof. One has (3.19) and (3.20) by applying (3.15) with $k, l=1,2, j=3$ and with $l=1,2, k, j=3$, respectively.

\section{Split wave Propagation over a Boltzmann Shock Profile:}

\section{$\mathbb{T}$ - $\mathbb{C}$ SCHEME}

Consider the linearized Boltzmann equation,

$$
\partial_{t} \mathrm{f}+\xi^{1} \partial_{x} \mathrm{f}=\mathrm{L}_{\varphi} \mathrm{f}
$$

around a stationary Boltzmann shock profile $\varphi(x)$ connecting $\left(\mathrm{M}_{-}, \mathrm{M}_{+}\right)$with $\mathrm{M}_{-}^{1 / 2}$ as a fixed reference Maxwellian, where the operator $\mathrm{L}_{\varphi}$ is an $x$-dependent linear operator in $L_{\xi}^{2}$ defined by

$$
\mathrm{L}_{\varphi} \mathrm{g} \equiv 2 \mathrm{M}_{-}^{-1 / 2} \mathrm{~B}\left(\varphi, \mathrm{M}_{-}^{\frac{1}{2}} \mathrm{~g}\right)
$$

4.1. A basic transverse initial value problem and outer approximation. The first step in performing the separation of scales is to decompose a distribution function $u$ according to the macroscopic hyperbolic equation (1.13) and the related macroscopic hyperbolic wave component $\mathrm{u}^{t}$ given in decomposition (1.15):

$$
\begin{cases}\mathrm{u}^{t} \equiv\left(\mathrm{I}_{1}, \mathrm{u}\right) \mathrm{r}_{1}+\left(\mathrm{I}_{2}, \mathrm{u}\right) \mathrm{r}_{2} & \text { (Transverse Component) } \\ \mathrm{u}^{c} \equiv\left(\mathrm{I}_{3}, \mathrm{u}\right) \mathrm{r}_{3} & \text { (Compressive Component) } \\ \mathrm{u}^{m} \equiv \mathrm{u}-\mathrm{u}^{t}-\mathrm{u}^{c} & \text { (Microscopic Component) }\end{cases}
$$

In this decomposition, transverse and compressive components are in the span of $\left\{\psi_{i} \sqrt{\mathrm{M}_{-}} \mid i=0,1,4\right\}$. Thus, one has the proposition on $\|\cdot\|_{L_{\xi}^{2}}$ and $\|\cdot\|_{L_{\xi, 2}^{\infty}}$ : 
Proposition 4.1. There exists $\mathscr{C}_{0}>0$ such that

$$
\left\{\begin{array}{l}
\frac{1}{\mathscr{C}_{0}}\left\|\mathrm{u}^{t}\right\|_{L_{\xi}^{2}} \leq\left\|\mathrm{u}^{t}\right\|_{L_{\xi, 3}^{\infty}} \leq \mathscr{C}_{0}\left\|\mathrm{u}^{t}\right\|_{L_{\xi}^{2}}, \\
\frac{1}{\mathscr{C}_{0}}\left\|\mathrm{u}^{c}\right\|_{L_{\xi}^{2}} \leq\left\|\mathrm{u}^{c}\right\|_{L_{\xi, 3}^{\infty}} \leq \mathscr{C}_{0}\left\|\mathrm{u}^{c}\right\|_{L_{\xi}^{2}} .
\end{array}\right.
$$

This yields that the norms $\|\cdot\|_{L_{\xi}^{2}}$ and $\|\cdot\|_{L_{\xi, 3}^{\infty}}$ are equivalent norms on the transverse and compressive components.

For the purpose of modeling the transverse wave propagation across the shock front, we impose the initial data satisfying $\mathrm{g}(x, 0)^{m}=\mathrm{g}(x, 0)^{c} \equiv 0$ first:

$$
\left\{\begin{array}{l}
\partial_{t} \mathrm{~g}+\xi^{1} \partial_{x} \mathrm{~g}-\mathrm{L}_{\varphi} \mathrm{g}=0 \\
\mathrm{~g}(x, 0, \xi)=a\left(x ; y_{0}\right) \mathrm{r}_{1}+b\left(x ; y_{0}\right) \mathrm{r}_{2}
\end{array}\right.
$$

where

$$
a\left(x, y_{0}\right), b\left(x, y_{0}\right)=\left\{\begin{array}{l}
0 \text { for }\left|x-y_{0}\right| \geq 1 \\
O(1) \text { otherwise }
\end{array}\right.
$$

where $y_{0}$ is a parameter for modeling the location of the initial data. First, we consider the case

$$
y_{0}=0 .
$$

It corresponds to the case where the initial data is contained inside a shock layer.

For this initial value problem, one introduces two functions $\mathrm{g}_{O}^{ \pm}(x, t)$ to model wave propagation at $x= \pm \infty$ :

$$
\mathrm{g}_{O}^{ \pm}(x, t) \equiv \int_{\mathbb{R}} \mathbb{G}_{ \pm}(x-y, t) \mathrm{g}(y, 0) d y .
$$

Estimate (3.11), Corollary 2.11, Corollary 3.1, and Corollary 3.3 together yield that for $|x| \leq 2 c(t+1)$

$$
\begin{aligned}
& \left\|\mathrm{g}_{O}^{+}(x, t)\right\|_{L_{\xi}^{2}} \leq O(1)\left[\sum_{j=1}^{2} \frac{e^{-\frac{\left(x-\lambda_{j}^{+} t\right)^{2}}{D_{0}(1+t)}}}{\sqrt{1+t}}+\left(\varepsilon+\frac{1}{\sqrt{1+t}}\right) \frac{e^{-\frac{\left(x-\lambda_{3}^{+} t\right)^{2}}{D_{0}(1+t)}}}{\sqrt{1+t}}\right], \\
& \left\|\mathrm{g}_{O}^{-}(x, t)\right\|_{L_{\xi}^{2}} \leq O(1)\left[\sum_{j=1}^{2} \frac{e^{-\frac{\left(x-\lambda_{j}^{-} t\right)^{2}}{D_{0}(1+t)}}}{\sqrt{1+t}}+\frac{e^{-\frac{\left(x-\lambda_{3}^{-} t\right)^{2}}{D_{0}(1+t)}}}{1+t}\right], \\
& \left\|\mathrm{P}_{1}^{-} \mathrm{g}_{O}^{+}(x, t)\right\|_{L_{\xi}^{2}} \leq O(1)\left[\sum_{j=1}^{2} \frac{e^{-\frac{\left(x-\lambda_{j}^{+} t\right)^{2}}{D_{0}(1+t)}}}{1+t}+\left(\varepsilon+\frac{1}{\sqrt{1+t}}\right) \frac{e^{-\frac{\left(x-\lambda_{3}^{+} t\right)^{2}}{D_{0}(1+t)}}}{1+t}\right], \\
& \left\|\mathrm{P}_{1}^{-} \mathrm{g}_{O}^{-}(x, t)\right\|_{L_{\xi}^{2}} \leq O(1)\left[\sum_{j=1}^{2} \frac{e^{-\frac{\left(x-\lambda_{j}^{-} t\right)^{2}}{D_{0}(1+t)}}}{1+t}+\frac{e^{-\frac{\left(x-\lambda_{3}^{-} t\right)^{2}}{D_{0}(1+t)}}}{(1+t)^{3 / 2}}\right] .
\end{aligned}
$$


From (3.19) and (3.20) one has that for some $D_{1}>4 D_{0}$ and $E_{0}>1$

$$
\left\{\begin{array}{l}
e^{-\frac{4 \varepsilon|x|}{D_{1}}}\left\|\mathrm{E}_{k}^{-, *}\left(\mathrm{~g}_{O}^{+}-\mathrm{g}_{O}^{-}\right)\right\|_{L_{\xi}^{2}} \\
\quad \leq O(1)\left(\left(\frac{\varepsilon}{1+t}+\frac{\varepsilon^{2}}{(1+t)^{1 / 2}}\right) e^{-\varepsilon^{2} t / E_{0}}+\frac{\sqrt{\varepsilon}}{(1+t)^{1 / 2}} e^{-\varepsilon t / E_{0}}\right) e^{-\frac{3 \varepsilon|x|}{D_{1}}}, \\
e^{-\frac{4 \varepsilon|x|}{D_{1}}}\left\|\mathrm{E}_{3}^{-, *}\left(\mathrm{~g}_{O}^{+}-\mathrm{g}_{O}^{-}\right)\right\|_{L_{\xi}^{2}} \leq O(1) \varepsilon \frac{e^{-\frac{3 \varepsilon|x|}{D_{1}}-\frac{\varepsilon^{2} t}{E_{0}}}+e^{-\frac{3 \varepsilon|x|}{D_{1}}-\frac{\varepsilon t}{E_{0}}}}{\sqrt{1+t}}
\end{array}\right.
$$

for $k=1,2$. Similarly, by applying (3.19), (3.20), and (3.16), one has that

$$
\begin{aligned}
& e^{-\frac{4 \varepsilon|x|}{D_{1}}}\left\|\mathrm{P}_{1}^{-}\left(\mathrm{g}_{O}^{-}-\mathrm{g}_{O}^{+}\right)\right\|_{L_{\xi}^{2}} \\
& \quad \leq O(1)\left(\left(\frac{\varepsilon}{1+t}+\frac{\varepsilon^{2}}{(1+t)^{1 / 2}}\right) e^{-\varepsilon^{2} t / E_{0}}+\frac{\varepsilon}{(1+t)^{1 / 2}} e^{-\varepsilon t / E_{0}}\right) e^{-\frac{3 \varepsilon|x|}{D_{1}}} .
\end{aligned}
$$

By a standard bootstrap procedure, which can be found in Section 7.1 of [13], one can improve the $\|\cdot\|_{L_{\xi}^{2} \text {-estimate in (4.12) }}$ to the $\|\cdot\|_{L_{\xi, 3}^{\infty}}$-estimate

$$
\begin{aligned}
& e^{-\frac{4 \varepsilon|x|}{D_{1}}}\left\|\mathrm{P}_{1}^{-}\left(\mathrm{g}_{O}^{-}-\mathrm{g}_{O}^{+}\right)\right\|_{L_{\xi, 3}^{\infty}} \\
& \quad \leq O(1)\left(\left(\frac{\varepsilon}{1+t}+\frac{\varepsilon^{2}}{(1+t)^{1 / 2}}\right) e^{-\varepsilon^{2} t / E_{0}}+\frac{\varepsilon}{(1+t)^{1 / 2}} e^{-\varepsilon t / E_{0}}\right) e^{-\frac{3 \varepsilon|x|}{D_{1}}} .
\end{aligned}
$$

We introduce a partition of unit $\left\{\chi_{-}, \chi_{+}\right\}$. The size of the compact support of this partition of unit is subject to the viscosity coefficient in the Green's function and the structure of the Boltzmann shock profile $\varphi$ given in (2.13) as follows:

$$
\begin{aligned}
& \chi_{-}(x)= \begin{cases}1 & \text { for } x<-\varepsilon^{-1} D_{0} / 4, \\
0 & \text { for } x>\varepsilon^{-1} D_{0} / 4,\end{cases} \\
& \chi_{+}(x)=1-\chi_{-}(x), \\
& 0<\chi_{-}^{\prime}(x) \leq O(1) \varepsilon^{2} e^{-\frac{4 \varepsilon|x|}{D_{0}}},
\end{aligned}
$$

where the constant $D_{0}$ is specified in Lemma 3.4 and $\varepsilon$ is the shock strength of $\left(\overrightarrow{\boldsymbol{u}}_{-}, \overrightarrow{\boldsymbol{u}}_{+}\right)$.

An outer approximation is defined by

$$
\begin{aligned}
& \mathrm{g}_{O}(x, t) \\
& \equiv\left\{\begin{array}{l}
\chi_{-}(x) \mathrm{g}_{O}^{-}(x, t)+\chi_{+}(x) \mathrm{g}_{O}^{+}(x, t) \text { for } t \geq 2 \tau_{0} \varepsilon^{-2} \\
\left.\begin{array}{l}
\varepsilon^{2} \\
\tau_{0}
\end{array}\left(2 \tau_{0} \varepsilon^{-2}-t\right) \chi_{-}(x) \mathrm{g}_{O}^{-}(x, t)+\left(t-\tau_{0} \varepsilon^{-2}\right) \chi_{+}(x) \mathrm{g}_{O}^{+}(x, t)\right) \\
\text { for } t \in\left(\tau_{0} \varepsilon^{-2}, 2 \tau_{0} \varepsilon^{-2}\right), \\
\mathrm{g}_{O}^{-}(x, t) \text { for } t \in\left(0, \tau_{0} \varepsilon^{-2}\right),
\end{array}\right.
\end{aligned}
$$

where the number $\tau_{0}>0$ satisfies

$$
\left\{\begin{array}{l}
\tau_{0} \ll 1 \\
e^{-\frac{\left(x-\tau_{0} \varepsilon^{-1}\right)^{2}}{D_{0} \tau_{0} \varepsilon^{-2}}} \leq 2 e^{-\frac{4 \varepsilon|x|}{D_{0}}} .
\end{array}\right.
$$


This outer approximation is an exact solution for $t=0$ and for $x= \pm \infty$ as $t \geq$ $O(1) \varepsilon^{-2}$.

The truncation error $\mathrm{er}_{1}(x, t)$ of the approximation $\mathrm{g}_{O}(x, t)$ is

$$
\operatorname{er}_{1}(x, t) \equiv-\left(\partial_{t} \mathrm{~g}_{O}+\xi^{1} \partial_{x} \mathrm{~g}_{O}-\mathrm{L}_{\varphi} \mathrm{g}_{O}\right) .
$$

Lemma 4.2. For given $D_{1}>2 D_{0}$ and $\tau_{0}$ satisfying (4.15), the truncation error $\mathrm{er}_{1}$ in the above satisfies the following cases.

Case $t \in\left(0, \tau_{0} \varepsilon^{-2}\right)$.

$$
\begin{aligned}
\mathrm{P}_{0}^{-} \operatorname{er}_{1}(x, t) & \equiv 0, \\
\left\|\mathrm{P}_{1}^{-} \operatorname{er}_{1}(x, t)\right\|_{L_{\xi}^{2}} & \leq O(1) \varepsilon\left(\frac{e^{-\frac{\varepsilon t}{E_{0}}}}{\sqrt{(1+t)}}+\frac{e^{-\frac{\varepsilon^{2} t}{E_{0}}}}{(1+t)}\right) e^{-\frac{3 \varepsilon|x|}{D_{1}}} .
\end{aligned}
$$

Case $t \in\left(\tau_{0} \varepsilon^{-2}, 2 \tau_{0} \varepsilon^{-2}\right)$.

$$
\left\{\begin{array}{l}
\left\|\mathrm{P}_{0}^{-} \operatorname{er}_{1}(x, t)\right\|_{L_{\xi}^{2}}=O(1) \varepsilon^{2} \frac{e^{-\frac{3 \varepsilon|x|}{D_{1}}-\frac{\varepsilon^{2} t}{E_{0}}}}{(1+t)} \\
\left\|\mathrm{P}_{1}^{-} \operatorname{er}_{1}(x, t)\right\|_{L_{\xi}^{2}}=O(1) \varepsilon \frac{e^{-\frac{3 \varepsilon|x|}{D_{1}}-\frac{\varepsilon^{2} t}{E_{0}}}}{(1+t)}
\end{array}\right.
$$

Case $t>2 \tau_{0} \varepsilon^{-2}$.

$$
\begin{gathered}
\left\|\mathrm{P}_{0}^{-} \operatorname{er}_{1}(x, t)\right\|_{L_{\xi}^{2}}=O(1) \varepsilon^{2} \frac{e^{-\frac{3 \varepsilon|x|}{D_{1}}-\frac{\varepsilon^{2} t}{E_{0}}}}{(1+t)}, \\
\left\|\mathrm{P}_{1}^{-} \operatorname{er}_{1}(x, t)\right\|_{L_{\xi}^{2}}=O(1) \varepsilon \frac{e^{-\frac{3 \varepsilon|x|}{D_{1}}-\frac{\varepsilon^{2} t}{E_{0}}}}{(1+t)} .
\end{gathered}
$$

Proof. From the definition (4.16),

$$
\operatorname{er}_{1}=\left\{\begin{array}{l}
\left(\mathrm{L}_{\varphi}-\mathrm{L}_{-}\right) \mathrm{g}_{O}^{-} \text {for } t \in\left(0, \tau_{0} \varepsilon^{-2}\right), \\
\frac{\varepsilon^{2}}{\tau_{0}} \chi_{+}\left(\mathrm{g}_{O}^{-}-\mathrm{g}_{O}^{+}\right)+\frac{\varepsilon^{2}}{\tau_{0}}\left(2 \tau_{0} \varepsilon^{-2}-t\right)\left[\chi_{-}\left(\mathrm{L}_{\varphi}-\mathrm{L}_{-}\right) \mathrm{g}_{O}^{-}-\xi^{1} \chi_{-}^{\prime} \mathrm{g}_{O}^{-}\right] \\
+\frac{\varepsilon^{2}}{\tau_{0}}\left(t-\tau_{0} \varepsilon^{-2}\right)\left[\chi_{+}\left(\mathrm{L}_{\varphi}-\mathrm{L}_{+}\right) \mathrm{g}_{O}^{+}-\xi^{1} \chi_{-}^{\prime}\left(\mathrm{g}_{O}^{-}-\mathrm{g}_{O}^{+}\right)\right] \text {for } t \in\left(\tau_{0} \varepsilon^{-2}, 2 \tau_{0} \varepsilon^{-2}\right), \\
\chi_{+}\left(\mathrm{L}_{\varphi}-\mathrm{L}_{+}\right) \mathrm{g}_{O}^{+}+\chi_{-}\left(\mathrm{L}_{\varphi}-\mathrm{L}_{-}\right) \mathrm{g}_{O}^{-}-\xi^{1} \chi_{-}^{\prime}\left(\mathrm{g}_{O}^{-}-\mathrm{g}_{O}^{+}\right) .
\end{array}\right.
$$

Case $t \in\left(0,2 \tau_{0} \varepsilon^{-2}\right)$.

Due to the fact that both the images of $\mathrm{L}_{\varphi}$ and $\mathrm{L}_{-}$are purely microscopic and due to (4.22), one has (4.17).

From (4.7) and (4.22),

$$
\begin{array}{r}
\left\|\mathrm{P}_{1}^{-} \operatorname{er}_{1}(x, t)\right\|_{L_{\xi}^{2}}=\left\|\left(\mathrm{L}_{\varphi}-\mathrm{L}_{-}\right) \mathrm{g}_{O}^{-}\right\|_{L_{\xi}^{2}} \leq O(1)\left\|\varphi(x)-\mathrm{M}_{-}\right\|_{L_{\xi}^{2}}\left\|\mathrm{~g}_{O}^{-}(x, t)\right\|_{L_{\xi}^{2}} \\
\leq O(1) \varepsilon\left(\frac{e^{-\frac{\varepsilon t}{E_{0}}}}{\sqrt{(1+t)}}+\frac{e^{-\frac{\varepsilon^{2} t}{E_{0}}}}{(1+t)}\right) e^{-\frac{3 \varepsilon|x|}{D_{1}}} .
\end{array}
$$


Case $t \geq 2 \tau_{0} \varepsilon^{-2}$.

From (4.22), (4.12), and (4.11), it follows that

$$
=\left|\chi_{-}^{\prime}(x)\right|\left\|\sum_{j=1}^{3} \lambda_{j}^{-} \mathrm{E}_{j}^{-, *}\left(\mathrm{~g}_{O}^{-}-\mathrm{g}_{O}^{+}\right)+\mathrm{P}_{0}^{-} \xi^{1} \mathrm{P}_{1}^{-}\left(\mathrm{g}_{O}^{+}-\mathrm{g}_{O}^{-}\right)\right\|_{L_{\xi}^{2}} \leq O(1) \frac{\varepsilon^{2}}{(1+t)} e^{-\frac{3 \varepsilon|x|}{D_{1}}-\frac{\varepsilon^{2} t}{E_{0}}} ;
$$

and (4.22), (4.12), (4.11), (4.8), and (4.7) together yield

$$
\left\|\mathrm{P}_{1}^{-} \operatorname{er}_{1}(x, t)\right\|_{L_{\xi}^{2}} \leq\left|\chi_{-}^{\prime}(x)\right|\left\|\mathrm{P}_{1}^{-} \xi^{1}\left(\mathrm{~g}_{O}^{+}-\mathrm{g}_{O}^{-}\right)\right\|_{L_{\xi}^{2}}
$$

$$
+\chi_{+}(x)\left\|\left(\mathrm{L}_{\varphi}-\mathrm{L}_{+}\right) \mathrm{g}_{O}^{+}\right\|_{L_{\xi}^{2}}+\chi_{-}(x)\left\|\left(\mathrm{L}_{\varphi}-\mathrm{L}_{-}\right) \mathrm{g}_{O}^{-}\right\|_{L_{\xi}^{2}} \leq O(1) \frac{\varepsilon}{(1+t)} e^{-\frac{3 \varepsilon|x|}{D_{1}}-\frac{\varepsilon^{2} t}{E_{0}}} .
$$

Case $t \in\left(\tau_{0} \varepsilon^{-2}, 2 \tau_{0} \varepsilon^{-2}\right)$.

From the expression of the truncation error $\mathrm{er}_{1}$ in (4.22) in the time interval $t \in\left(2 \tau_{0} \varepsilon^{-2}, 3 \tau_{0} \varepsilon^{-2}\right)$, it is sufficient to estimate $\frac{\varepsilon^{2}}{\tau_{0}} \chi_{+}(x)\left(\mathrm{g}_{O}^{-}(x, t)-\mathrm{g}_{O}^{+}(x, t)\right)$ in the time interval $\left(\tau_{0} \varepsilon^{-2}, 2 \tau_{0} \varepsilon^{-2}\right)$.

With the properties $\chi_{+}(x)<e^{-4 \frac{\varepsilon|x|}{D_{0}}}$ for $x<0$ and $e^{-\frac{(x-\varepsilon t)^{2}}{D_{0} t}} \leq 2 e^{-4 \varepsilon|x| / D_{0}}$ for $t \in\left(\tau_{0} \varepsilon^{-2}, 2 \tau_{0} \varepsilon^{-2}\right)$, one has

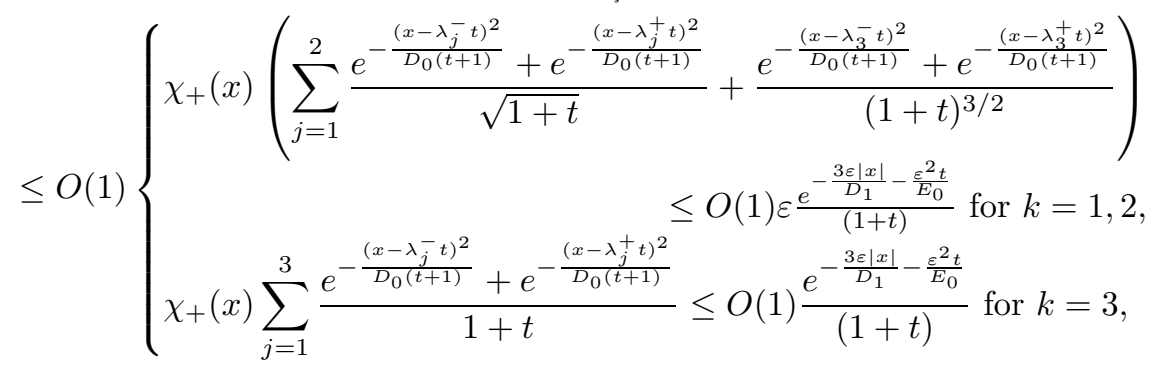

and

$$
\left\|\chi_{+}(x) \mathrm{P}_{1}^{-}\left(\mathrm{g}_{O}^{-}(x, t)-\mathrm{g}_{O}^{+}(x, t)\right)\right\|_{L_{\xi}^{2}} \leq O(1) \varepsilon \frac{e^{-\frac{3 \varepsilon|x|}{D_{1}}-\frac{\varepsilon^{2} t}{E_{0}}}}{1+t} .
$$

Thus, from (4.22) and from the interpolation of (4.25), (4.24), (4.23), (4.17) with (4.26) and (4.27), one has (4.19).

Remark 4.3. Due to both the facts that the initial data $\mathrm{g}(x, 0)$ of (4.5) satisfies $\|\mathrm{g}(x, 0)\|_{L_{\varepsilon, 3}^{\infty}} \leq O(1)\left(\left|a\left(x ; y_{0}\right)\right|+\left|b\left(x ; y_{0}\right)\right|\right)$ and the factor $\mathrm{L}_{\varphi}-\mathrm{L}_{-}$contains a factor $O(1)(1+|\xi|)$, the truncation error er ${ }_{1}$ satisfies

$$
\left\{\begin{array}{l}
\mathrm{P}_{0}^{-} \operatorname{er}_{1}(x, t) \equiv 0,\left\|\mathrm{P}_{1}^{-} \operatorname{er}_{1}(x, t)\right\|_{L_{\xi, 2}^{\infty}} \\
\quad \leq O(1) \varepsilon\left(\frac{e^{-\frac{\varepsilon t}{E_{0}}}}{\sqrt{(1+t)}}+\frac{e^{-\frac{\varepsilon^{2} t}{E_{0}}}}{(1+t)}\right)^{-\frac{3 \varepsilon|x|}{D_{1}}} \text { for } t \in\left(0, \tau_{0} \varepsilon^{-2}\right), \\
\left\|\mathrm{P}_{0}^{-} \operatorname{er}_{1}(x, t)\right\|_{L_{\xi, 3}^{\infty}}=O(1) \varepsilon^{2} \frac{e^{-\frac{3 \varepsilon|x|}{D_{1}}-\frac{\varepsilon^{2} t}{E_{0}}}}{(1+t)} \text { for } t \in\left(\tau_{0} \varepsilon^{-2}, \infty\right), \\
\left\|\mathrm{P}_{1}^{-} \operatorname{er}_{1}(x, t)\right\|_{L_{\xi, 2}^{\infty}}^{\infty}=O(1) \varepsilon \frac{e^{-\frac{3 \varepsilon|x|}{D_{1}}-\frac{\varepsilon^{2} t}{E_{0}}}}{(1+t)} \text { for } t \in\left(\tau_{0} \varepsilon^{-2}, \infty\right) .
\end{array}\right.
$$


4.2. The $\mathbb{T}-\mathbb{C}$ scheme: Transverse wave operators, compressive wave operators, and local wave front tracing. For the approximate solution $\mathrm{g}_{O}(x, t)$ to the solution $\mathrm{g}$ of (4.5), the error function $\mathrm{h} \equiv \mathrm{g}-\mathrm{g}_{O}$ satisfies

$$
\left\{\begin{array}{l}
\left(\partial_{t}+\xi^{1} \partial_{x}-\mathrm{L}_{\varphi}\right) \mathrm{h}=\mathrm{er}_{1} \\
\mathrm{~h}(x, 0) \equiv 0
\end{array}\right.
$$

We rewrite this problem in a general form with er 1 replaced by a given inhomogeneous term ER:

$$
\left\{\begin{array}{l}
\left(\partial_{t}+\xi^{1} \partial_{x}-\mathrm{L}_{\varphi}\right) \mathrm{H}=\mathrm{ER} \\
\mathrm{H}(x, 0) \equiv 0
\end{array}\right.
$$

The Transverse Wave Operator

With the given inhomogeneous term ER, we introduce two sequences $\left\{\mathrm{ER}_{j}\right\}_{j \in \mathbb{N}}$ and $\left\{G_{j}\right\}_{j \geq 2}$ given by the recursive procedure together with the decomposition (1.15):

$$
\begin{aligned}
\mathrm{ER}_{1} & \equiv \mathrm{ER} . \\
\mathrm{G}_{j+1}^{ \pm}(x, t) & \equiv \int_{0}^{t} \int_{\mathbb{R}} \mathbb{G}_{ \pm}(x-y, t-s) \operatorname{ER}_{j}^{t}(y, s) d y d s \\
\mathrm{G}_{j+1}(x, t) & \equiv \chi_{-}(x) \mathrm{G}_{j+1}^{-}(x, t)+\chi_{+}(x) \mathrm{G}_{j+1}^{+}(x, t), \\
\operatorname{ER}_{j+1}(x, t) & \equiv-\left(\partial_{t}+\xi^{1} \partial_{x}-\mathrm{L}_{\varphi}\right) \mathrm{G}_{j+1}(x, t)+\operatorname{ER}_{j}^{t}(x, t) .
\end{aligned}
$$

The above sequence defines the transverse wave operator $\mathbb{T}[E R]$ :

$$
\mathbb{T}[\mathrm{ER}](x, t) \equiv \sum_{j=2}^{\infty} \mathrm{G}_{j}(x, t)
$$

Then the operator $\mathbb{T}$ depends on the transverse component $\mathrm{ER}^{t}$ only:

$$
\left\{\begin{array}{l}
\mathbb{T}\left[\mathrm{ER}^{t}\right]=\mathbb{T}[\mathrm{ER}] \\
\mathbb{T}\left[\mathrm{ER}^{c}\right]=0 \\
\mathbb{T}\left[\mathrm{ER}^{m}\right]=0
\end{array}\right.
$$

\section{Local Wave Front Tracing}

The function $\mathbb{T}[E R]$ in (4.34) gives an approximate solution of (4.30) without generating errors in the transverse component:

$$
\left(\partial_{t}+\xi^{1} \partial_{x}-\mathrm{L}_{\varphi}\right) \mathbb{T}[\mathrm{ER}](x, t)-\operatorname{ER}(x, t)=-\sum_{j=1}^{\infty}\left(\operatorname{ER}_{j}^{c}(x, t)+\operatorname{ER}_{j}^{m}(x, t)\right)
$$

The truncation errors are the sum of the compressive wave component $-\sum_{j=1}^{\infty} \mathrm{ER}_{j}^{c}$ and the microscopic component $-\sum_{j=1}^{\infty} \mathrm{ER}_{j}^{m}$. The compressive component breaks the macroscopic conservation laws. One introduces a local wave front tracing to recover the macroscopic conservation laws. The procedure for building the local 
wave front trace is

$$
\begin{aligned}
d_{j}(t) & \equiv \int_{0}^{t}\left(\mathrm{I}_{3},\left(\int_{\mathbb{R}} \mathrm{ER}_{j}^{c}(z, s) d z\right)\right) d s \\
d(t) \equiv & \sum_{j=1}^{\infty} d_{j}(t) \\
\mathbb{D}[\mathrm{ER}](x, t) \equiv & \frac{d(t)}{\left(\mathrm{I}_{3},\left(\mathrm{M}_{+}-\mathrm{M}_{-}\right) / \mathrm{M}_{-}^{1 / 2}\right)} \varphi^{\prime}(x) \text { (the local wave front component). }
\end{aligned}
$$

A residue operator $\mathbb{R}_{3}[E R]$ is defined by

$$
\mathbb{R}_{3}[\mathrm{ER}](x, t) \equiv \sum_{j=1}^{\infty}\left(\mathrm{ER}_{j}^{m}+\operatorname{ER}_{j}^{c}(x, t)\right)-\frac{d^{\prime}(t)}{\left(\mathrm{I}_{3},\left(\mathrm{M}_{+}-\mathrm{M}_{-}\right) / \mathrm{M}_{-}^{\frac{1}{2}}\right)} \varphi^{\prime}(x)
$$

so that the total macroscopic mass of $\mathbb{R}_{3}[E R]$ is zero:

$$
\begin{aligned}
& \mathrm{P}_{0}^{-} \int_{\mathbb{R}} \mathbb{R}_{3}[\mathrm{ER}](x, t) d x \\
&=\mathrm{P}_{0}^{-} \int_{\mathbb{R}}\left\{\sum_{j=1}^{\infty}\left(\mathrm{ER}_{j}^{m}(x, t)+\mathrm{ER}_{j}^{c}(x, t)\right)-\frac{d^{\prime}(t) \varphi^{\prime}(x)}{\left(\mathrm{I}_{3},\left(\mathrm{M}_{+}-\mathrm{M}_{-}\right) / \mathrm{M}_{-}^{1 / 2}\right)}\right\} d x \\
&=\mathrm{P}_{0}^{-} \int_{\mathbb{R}}\left(\sum_{j=1}^{\infty} \mathrm{ER}_{j}^{c}(x, t)-\frac{d^{\prime}(t) \varphi^{\prime}(x)}{\left(\mathrm{I}_{3},\left(\mathrm{M}_{+}-\mathrm{M}_{-}\right) / \mathrm{M}_{-}^{1 / 2}\right)}\right) d x \\
&=\int_{\mathbb{R}} \sum_{j=1}^{\infty} \mathrm{ER}_{j}^{c}(x, t) d x-d^{\prime}(t) \mathrm{r}_{3}=0 .
\end{aligned}
$$
by

$$
\mathbb{R}_{3}[\mathrm{ER}] \equiv-\left(\partial_{t}+\xi^{1} \partial_{x}-\mathrm{L}_{\varphi}\right)(\mathbb{T}+\mathbb{D})[\mathrm{ER}]+\mathrm{ER}
$$

Thus, from (4.40) and (4.39), one recovers the macroscopic conservation laws:

$$
\mathrm{P}_{0}^{-}\left(\int_{\mathbb{R}}\left(\partial_{t}+\xi^{1} \partial_{x}-\mathrm{L}_{\varphi}\right)(\mathbb{T}+\mathbb{D})[\mathrm{ER}](x, t)-\operatorname{ER}(x, t) d x\right) \equiv 0 \text { for } t \geq 0 .
$$

\section{Compressive Wave Operator}

From (4.40), the error function $\mathrm{c}(x, t)$ of the approximate function $(\mathbb{T}+\mathbb{D})[\mathrm{ER}]$ to (4.30) satisfies the initial value problem

$$
\left\{\begin{array}{l}
\left(\partial_{t}+\xi^{1} \partial_{x}-\mathrm{L}_{\varphi}\right) \mathrm{c}=\mathbb{R}_{3}[\mathrm{ER}] \\
\mathrm{c}(x, 0) \equiv 0
\end{array}\right.
$$

In the solution $\mathrm{c}(x, t)$, there are slowly decaying waves propagating in different directions. This multi-direction propagation prevents one from obtaining any exponentially sharp structure directly. However, in $\mathbb{R}_{3}[E R]$ there is no transverse component so that one can trade off some minor errors in the transverse component to gain exponentially sharp estimates in the compressive component around 
the shock front. This leads to the compressive operator $\mathbb{C}_{3}[S]$ possessing an exponentially sharp pointwise structure for any given input function $\mathrm{S}(x, t) \equiv \mathbb{R}_{3}[\mathrm{ER}]$. The function $\mathbb{C}_{3}[S](x, t)$ is defined by the solution,

$$
\mathbb{C}_{3}[\mathrm{~S}](x, t) \equiv \mathrm{C}(x, t) \text { (Compressive Wave Operator), }
$$

of an initial value problem with a given inhomogeneous term $\mathrm{S}$

$$
\left\{\begin{array}{l}
\partial_{t} \mathrm{C}+\xi^{1} \partial_{x} \mathrm{C}-\mathrm{L}_{\varphi} \mathrm{C}+\varepsilon \sum_{j=1}^{2}\left(\mathrm{I}_{j}, \mathrm{C}\right) \mathrm{r}_{j}=\mathrm{S} \\
\mathrm{C}(x, 0) \equiv 0
\end{array}\right.
$$

Here index 3 is referred to as the compressive field in the 3 -characteristic field. Then one uses

$$
\mathrm{T}[\mathrm{ER}](x, t) \equiv\left(\mathbb{T}+\mathbb{D}+\mathbb{C}_{3} \circ \mathbb{R}_{3}\right)[\mathrm{ER}](x, t)
$$

to construct an improved approximate solution to (4.30):

$$
\begin{aligned}
\left(\partial_{t}+\xi^{1} \partial_{x}-\mathrm{L}_{\varphi}\right)\left(\mathbb{T}+\mathbb{D}+\mathbb{C}_{3} \circ \mathbb{R}_{3}\right)[\mathrm{ER}]-\mathrm{ER} \\
=-\varepsilon \sum_{l=1}^{2}\left(\mathrm{I}_{j}, \mathbb{C}_{3}\left[\mathbb{R}_{3}[\mathrm{ER}]\right]\right) \mathrm{r}_{j} \text { (purely transverse component). }
\end{aligned}
$$

The truncation error, $-\mathfrak{R}[\mathrm{ER}]$, of the improved approximate solution $\mathrm{T}[\mathrm{ER}]$ is

$$
\mathfrak{R}[\mathrm{ER}](x, t) \equiv \varepsilon \sum_{j=1}^{2}\left(\mathrm{I}_{j}, \mathbb{C}_{3}\left[\mathbb{R}_{3}[\mathrm{ER}]\right](x, t)\right) \mathrm{r}_{j}
$$

Transverse-Compressive iteration scheme ( $\mathbb{T}-\mathbb{C}$ scheme)

With the operators $T$ and $\mathfrak{R}$, we introduce the $\mathbb{T}$ - $\mathbb{C}$ scheme to construct the solution $\mathrm{H}(x, t)$ of (4.30). The sequence is

$$
\left\{\begin{array}{l}
\mathfrak{r}_{0} \equiv \mathrm{ER}, \\
\mathfrak{r}_{k} \equiv \mathfrak{R}\left[\mathfrak{r}_{k-1}\right] \text { for } k \geq 1, \\
\mathfrak{q}_{k} \equiv \mathrm{T}\left[\mathfrak{r}_{k-1}\right] \text { for } k \geq 1 .
\end{array}\right.
$$

From the relation of $\mathbb{T}, \mathbb{D}, \mathbb{C}_{3} \circ \mathbb{R}_{3}$, and $\mathfrak{R}$ in (4.46) and (4.40) one has

$$
\left(\partial_{t}+\xi^{1} \partial_{x}-\mathrm{L}_{\varphi}\right) \mathbf{q}_{k}-\mathfrak{r}_{k-1}=-\mathfrak{r}_{k} \text { for } k \geq 1 .
$$

This gives rise to the fact that

$$
\left(\partial_{t}+\xi^{1} \partial_{x}-\mathrm{L}_{\varphi}\right) \sum_{k=1}^{\infty} \mathrm{q}_{k}-\mathrm{ER}=-\lim _{k \rightarrow \infty} \mathfrak{r}_{k}
$$

Thus, if $\lim _{k \rightarrow \infty} \mathfrak{r}_{k}=0$, then $\sum_{k=1}^{\infty} \mathrm{q}_{k}$ solves $\mathrm{H}(x, t)$, which is the solution of (4.30), i.e.

$$
\mathrm{H}(x, t)=\sum_{k=1}^{\infty} \mathrm{q}_{k} .
$$

Here the elements $\mathfrak{r}_{j}$ and $\mathbf{q}_{j}$ can be expressed in the form $\mathfrak{r}_{j}[\mathrm{ER}]$ and $\mathbf{q}_{j}[\mathrm{ER}]$ to relate the dependence on ER, and the solution $g$ of (4.5) can be written formally in terms 
of the $\mathbb{T}$-C iteration scheme if $\lim _{k \rightarrow \infty} \mathfrak{r}_{k}=0$ :

$$
\mathrm{g}(x, t)=\mathrm{g}_{O}(x, t)+\sum_{j=1}^{\infty} \mathrm{q}_{j}\left[\operatorname{er}_{1}\right](x, t)
$$

4.3. Mild transverse initial data and the $\mathbb{T}-\mathbb{C}$ schemes. In (4.49), the function $\mathrm{g}_{O}(x, t)$ is devised for the purely transverse initial data in (4.5). When the total macroscopic mass of an initial data is zero, one uses a function $\mathbb{I}_{3}[\mathrm{~g}(\cdot, 0)](x, t)$ to replace the function $\mathrm{g}_{O}(x, t)$, where the operator $\mathbb{I}_{3}$ is defined by the solution of the following initial value problem:

$$
\begin{aligned}
& \mathbb{I}_{3}\left[\mathrm{~J}_{0}\right](x, t) \equiv \mathrm{J}(x, t), \\
& \left\{\begin{array}{l}
\left(\partial_{t}+\xi^{1} \partial_{x}-\mathrm{L}_{\varphi}\right) \mathrm{J}+\varepsilon \sum_{j=1}^{2}\left(\mathrm{I}_{j}, \mathrm{~J}\right) \mathrm{r}_{j}=0, \\
\mathrm{~J}(x, 0) \equiv \mathrm{J}_{0}(x) .
\end{array}\right.
\end{aligned}
$$

There are two other versions of the $\mathbb{T}-\mathbb{C}$ schemes subject to the type of the initial data.

Type I:

$$
\left\{\begin{array}{l}
\partial_{t} \mathrm{~g}_{I}+\xi^{1} \partial_{x} \mathrm{~g}_{I}-\mathrm{L}_{\varphi} \mathrm{g}_{I}=0 \\
\mathrm{~g}_{I}(x, 0)=c\left(x ; y_{0}\right) \mathrm{r}_{3}
\end{array}\right.
$$

Type II:

$$
\left\{\begin{array}{l}
\partial_{t} \mathrm{~g}_{I I}+\xi^{1} \partial_{x} \mathrm{~g}_{I I}-\mathrm{L}_{\varphi} \mathrm{g}_{I I}=0 \\
\mathrm{~g}_{I I}(x, 0)=\mathrm{d}\left(x ; y_{0}\right) \\
\mathrm{P}_{0}^{-} \mathrm{d} \equiv 0
\end{array}\right.
$$

The initial data $c\left(x ; y_{0}\right) \mathrm{r}_{3}$ and $\mathrm{d}\left(x ; y_{0}\right)$ satisfy

$$
\left\{\begin{array}{l}
c\left(x ; y_{0}\right),\left\|\mathrm{d}\left(x ; y_{0}\right)\right\|_{L_{\xi, 3}^{\infty}}=0 \text { for }\left|x-y_{0}\right| \geq 1, \\
c\left(x ; y_{0}\right),\left\|\mathrm{d}\left(x ; y_{0}\right)\right\|_{L_{\xi, 3}^{\infty}}^{\infty}=O(1) \text { for }\left|x-y_{0}\right| \leq 1 .
\end{array}\right.
$$

\section{Scheme for Type I.}

With the operators $\mathrm{T}$ and $\mathbb{I}_{3}$ given in (4.45) and (4.50), the scheme for $\mathrm{g}_{I}(x, t)$ is

$$
\left\{\begin{array}{l}
\mathrm{g}_{I, 0}(x, t) \equiv \frac{\int_{-\infty}^{\infty} c\left(x ; y_{0}\right) d x}{\left(\mathrm{I}_{3},\left(\mathrm{M}_{+}-\mathrm{M}_{-}\right) / \sqrt{\mathrm{M}_{-}}\right)} \varphi^{\prime}(x), \\
\mathrm{q}_{0}(x, t) \equiv \mathbb{I}_{3}\left[\mathrm{~g}_{I}(\cdot, 0)-\mathrm{g}_{I, 0}(\cdot, 0)\right](x, t), \\
\mathfrak{r}_{0}(x, t) \equiv \varepsilon \sum_{j=1}^{2}\left(\mathrm{l}_{j}, \mathrm{q}_{0}(x, t)\right) \mathrm{r}_{j}, \\
\mathrm{q}_{i} \equiv \mathrm{T}\left[\mathfrak{r}_{i-1}\right], \mathfrak{r}_{i}=\mathfrak{R}\left[\mathfrak{r}_{i-1}\right] \text { for } i \geq 1 .
\end{array}\right.
$$


Here the operators $T$ and $\mathfrak{R}$ are given in (4.45) and (4.47). Similarly to (4.49), the solution $\mathrm{g}_{I}(x, t)$ can be formally expressed as the series:

$$
\mathrm{g}_{I}(x, t)=\mathrm{g}_{I, 0}(x, t)+\sum_{j=0}^{\infty} \mathrm{q}_{j}(x, t) .
$$

Here $\mathrm{g}_{I}(x, 0)-\mathrm{g}_{I, 0}(x)$ satisfies

$$
\left\{\begin{array}{l}
\int_{\mathbb{R}} \mathrm{P}_{0}^{-}\left(\mathrm{g}_{I}(x, 0)-\mathrm{g}_{I, 0}(x, 0)\right) d x=0 \\
\left|\left(\mathrm{I}_{j}, \mathrm{~g}_{I}(x, 0)-\mathrm{g}_{I, 0}(x)\right)\right| \leq O(1) \varepsilon^{2} e^{-4 \varepsilon|x| / D_{0}} \text { for } j=1,2 \text { and } x \in \mathbb{R}, \\
\left|\left(\mathrm{I}_{3}, \mathrm{~g}_{I}(x, 0)-\mathrm{g}_{I, 0}(x)\right)\right| \leq O(1) \varepsilon e^{-4 \varepsilon|x| / D_{0}} \text { for }|x| \geq 1 \\
\left\|\mathrm{~g}_{I}(x, 0)-\mathrm{g}_{I, 0}(x)\right\|_{L_{\xi, 3}^{\infty}}^{\infty}=O(1) \text { for }|x|<1 .
\end{array}\right.
$$

\section{Scheme for Type II.}

The scheme for $\mathrm{g}_{I I}(x, t)$ is

$$
\left\{\begin{array}{l}
\mathrm{q}_{0}(x, t) \equiv \mathbb{I}_{3}\left[\mathrm{~g}_{I I}(\cdot, 0)\right](x, t) \\
\mathfrak{r}_{0}(x, t) \equiv \varepsilon \sum_{j=1}^{2}\left(\mathrm{l}_{j}, \mathrm{q}_{0}(x, t)\right) \mathbf{r}_{j} \\
\mathbf{q}_{i} \equiv \mathrm{T}\left[\mathfrak{r}_{i-1}\right], \mathfrak{r}_{i}=\mathfrak{R}\left[\mathfrak{r}_{i-1}\right] \text { for } i \geq 1
\end{array}\right.
$$

Similarly to (4.49), formally the solution $\mathrm{g}_{I I}(x, t)$ is

$$
\mathrm{g}_{I I}(x, t)=\sum_{j=0}^{\infty} \mathrm{q}_{j}(x, t) .
$$

\section{ESTIMATES ON OPERATORS}

We begin with the introduction of the basic functions before we analyze the operators, $\mathbb{T}, \mathbb{R}_{3}, \mathbb{D}, \mathbb{C}_{3}, \mathbb{I}_{3}$, and $\mathrm{T}$ :

$$
\begin{aligned}
& \theta^{\alpha}(x, t ; \lambda, D) \equiv \frac{e^{-\frac{(x-\lambda t)^{2}}{D(1+t)}}}{(1+t)^{\frac{\alpha}{2}}}, \\
& \Gamma^{\alpha}(t) \equiv \int_{0}^{t}(s+1)^{-\alpha / 2} d s \\
& =O(1)\left\{\begin{array}{c}
1 \quad \text { for } \alpha>2, \\
\log (t+1) \text { for } \alpha=2, \\
(t+1)^{(2-\alpha) / 2} \text { for } \alpha<2 .
\end{array}\right.
\end{aligned}
$$

Since the transverse wave operator $\mathbb{T}$ depends on the transverse component $\mathrm{ER}^{t}$ only, while establishing the analysis of the operator $\mathbb{T}$, we impose the function $E R$ in the form with $\alpha \in[1,2]$ :

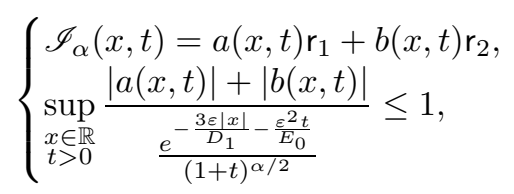




$$
\left\{\begin{array}{l}
\tilde{\mathscr{I}}_{\alpha}(x, t)=a(x, t) \mathbf{r}_{1}+b(x, t) \mathbf{r}_{2}, \\
\sup _{\substack{x \in \mathbb{R} \\
t>0}} \frac{|a(x, t)|+|b(x, t)|}{\frac{e^{-\frac{3 \varepsilon|x|}{D_{1}}-\frac{\varepsilon t}{E_{0}}}}{(1+t)^{\alpha / 2}}} \leq 1 .
\end{array}\right.
$$

The compressive operator $\mathbb{C}_{3}$ depends on both the microscopic component and the $r_{3}$ component and a multiple of $\varphi^{\prime}$. Thus, we need to consider ER in the following forms:

$$
\left\{\begin{array}{l}
\mathscr{U}_{\alpha}(x, t)=\left.a(x, t)\right|_{3}, \\
\tilde{\mathscr{U}}_{\alpha}(x, t)=\left.\tilde{a}(x, t)\right|_{3}, \\
\mathscr{V}_{\alpha}(x, t), \tilde{\mathscr{V}}_{\alpha}(x, t) \in \operatorname{Range}\left(\mathrm{P}_{1}^{-}\right), \\
\mathscr{W}_{\alpha}(x, t)=c_{\alpha}(t) \sum_{j=1}^{2} \frac{\left(\mathbf{I}_{j}, \varphi^{\prime}(x)\right) r_{j}}{\left\|\overrightarrow{\boldsymbol{u}}_{+}-\overrightarrow{\boldsymbol{u}}_{-}\right\|^{2}} \\
\tilde{\mathscr{W}}_{\alpha}=\tilde{c}_{\alpha}(t) \sum_{j=1}^{2} \frac{\left(\mathbf{I}_{j}, \varphi^{\prime}(x)\right) \mathrm{r}_{j}}{\left\|\overrightarrow{\boldsymbol{u}}_{+}-\overrightarrow{\boldsymbol{u}}_{-}\right\|^{2}}
\end{array}\right.
$$

where the functions $\mathscr{U}_{\alpha}, \tilde{\mathscr{U}}_{\alpha}, \mathscr{V}_{\alpha}, \tilde{\mathscr{V}}_{\alpha}, \mathscr{W}_{\alpha}$, and $\tilde{\mathscr{V}}_{\alpha}$ satisfy

$$
\begin{aligned}
& \int_{\mathbb{R}} a(x, t) d x=0, \int_{\mathbb{R}} \tilde{a}(x, t) d x=0 \text { for } t>0, \\
& \sup _{x \in \mathbb{R}, t \geq 0} \frac{|a(x, t)|+\|\mathscr{V}(x, t)\|_{L_{\xi, 2}^{\infty}}^{\infty}}{e^{-\frac{3 \varepsilon|x|}{D_{1}}-\frac{\varepsilon^{2} t}{E}}}+\sup _{x \in \mathbb{R}, t \geq 0} \frac{|\tilde{a}(x, t)|+\|\tilde{\mathscr{V}}(x, t)\|_{L_{\xi, 2}^{\infty}}^{\infty}}{\frac{e^{-\frac{3 \varepsilon|x|}{D_{1}}-\frac{\varepsilon t}{E}}}{(1+t)^{\frac{\alpha}{2}}}}<\infty, \\
& \sup _{t>0}\left|\frac{\left|c_{\alpha}(t)\right|}{\frac{e^{-\varepsilon^{2} t / E_{0}}}{(1+t)^{\alpha / 2}}}\right|+\left|\frac{\left|\tilde{c}_{\alpha}(t)\right|}{\frac{e^{-\varepsilon t / E_{0}}}{(1+t)^{\alpha / 2}}}\right|<\infty .
\end{aligned}
$$

\subsection{Estimates on the transverse wave operator.}

Lemma 5.1. For the function $\mathscr{I}_{\alpha}$ given in (5.2) with $\alpha \in[1,2]$, the function $\mathbb{T}\left[\mathscr{I}_{\alpha}\right](x, t)$ defined in (4.34) satisfies

$$
\begin{array}{r}
\left\|\mathbb{T}\left[\mathscr{I}_{\alpha}\right](x, t)\right\|_{L_{\xi, 3}^{\infty}} \leq O(1) \mathscr{A}_{\alpha}(x, t), \\
\left|\left(\mathrm{I}_{3}, \mathscr{I}_{\alpha}-\left(\partial_{t}+\xi^{1} \partial_{x}-\mathrm{L}_{\varphi}\right) \mathbb{T}\left[\mathscr{I}_{\alpha}\right]\right)\right| \leq O(1) \varepsilon \frac{e^{-\frac{3 \varepsilon|x|}{D_{1}}-\frac{\varepsilon^{2} t}{E_{0}}}}{(1+t)^{\frac{\alpha}{2}}}, \\
\left\|\left(\mathscr{I}_{\alpha}-\left(\partial_{t}+\xi^{1} \partial_{x}-\mathrm{L}_{\varphi}\right) \mathbb{T}\left[\mathscr{I}_{\alpha}\right]\right)^{m}\right\|_{L_{\xi, 2}^{\infty}} \leq O(1) \frac{e^{-\frac{3 \varepsilon|x|}{D_{1}}-\frac{\varepsilon^{2} t}{E_{0}}}}{(1+t)^{\frac{\alpha}{2}}} .
\end{array}
$$


Here the function $\mathscr{A}_{\alpha}(x, t)$ is defined by

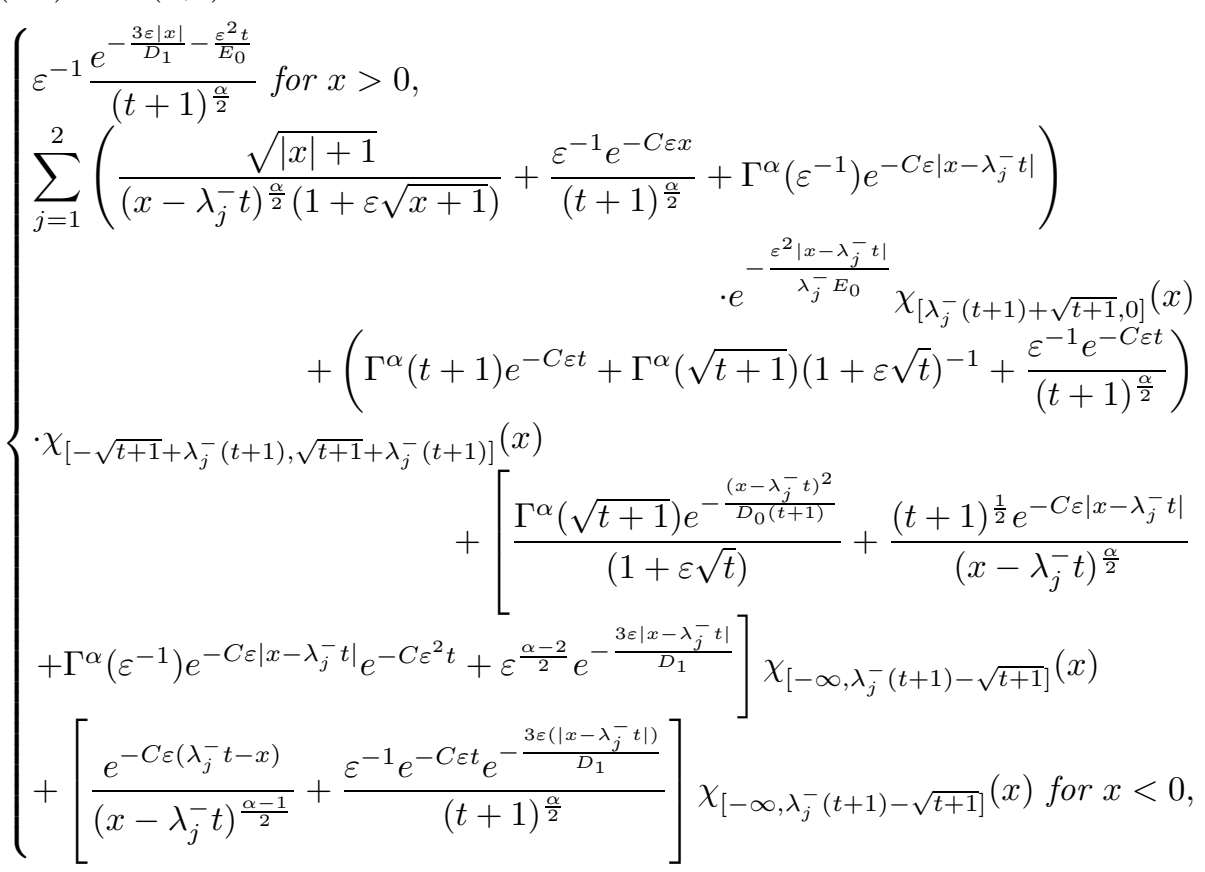

where $\chi_{[a, b]}$ is the characteristic function of the interval $[a, b]$, i.e.

$$
\chi_{[a, b]}(x)=1 \text { if } x \in[a, b] \text {, and otherwise } \chi_{[a, b]}(x)=0 .
$$

Proof. In the sequence $\left\{\mathrm{ER}_{j}\right\}_{j \in \mathbb{N}}$ in (4.33) for constructing $\mathbb{T}\left[\mathscr{I}_{\alpha}\right]$, the initial function $\mathrm{ER}_{1}$ is $\mathscr{I}_{\alpha}$. With $\mathrm{ER}_{1}$ determined, from (4.32), Corollary 2.11, Corollary 3.1. Corollary 3.3, and (3.11) together one has that

$$
\begin{gathered}
\cdot\left(\sum_{j=1}^{2} \int_{0}^{t} \int_{\mathbb{R}} \frac{\chi_{-}(x) e^{-\frac{\left(x-z-\lambda_{j}^{-}(t-s)\right)^{2}}{D_{0}(t-s+1)}}+\chi_{+}(x) e^{-\frac{\left(x-z-\lambda_{j}^{+}(t-s)\right)^{2}}{D_{0}(t-s+1)}}}{\sqrt{t-s+1}} \frac{e^{-\frac{\varepsilon^{2} s}{E_{0}}}}{(1+s)^{\alpha / 2}} e^{-\frac{3 \varepsilon|z|}{D_{1}}} d z d s\right. \\
+\int_{0}^{t} \int_{\mathbb{R}}\left(\varepsilon+\frac{1}{\sqrt{t-s+1}}\right) \frac{\chi_{-}(x) e^{-\frac{\left(x-z-\lambda_{3}^{-}(t-s)\right)^{2}}{D_{0}(t-s+1)}}+\chi_{+}(x) e^{-\frac{\left(x-z-\lambda_{3}^{+}(t-s)\right)^{2}}{D_{0}(t-s+1)}}}{\sqrt{t-s+1}} \\
\left.\cdot \frac{e^{-\frac{\varepsilon^{2} s}{E_{0}}}}{(1+s)^{\alpha / 2}} e^{-\frac{3 \varepsilon|z|}{D_{1}}} d z d s\right)
\end{gathered}
$$

Combine (5.10), (A.6) with $\beta=1$, (A.7), A.8) with $\beta=1,2$, and the property that

$$
\left\{\begin{array}{l}
\left|\lambda_{j}^{-}-\lambda_{j}^{+}\right|=O(1) \varepsilon \\
\left|\lambda_{j}^{ \pm}\right| \text {are away from zero for } j=1,2, \\
\mp \lambda_{3}^{ \pm}=O(1) \varepsilon>0
\end{array}\right.
$$


together to yield that

$$
\left\|\mathrm{G}_{2}(x, t)\right\|_{L_{\xi}^{2}} \leq O(1) \mathscr{A}_{\alpha}(x, t) \text { for } x \in \mathbb{R}
$$

The same argument yields

$$
\left\{\begin{array}{l}
\left\|\mathrm{G}_{2}^{-}(x, t)\right\|_{L_{\xi}^{2}} \leq O(1) \mathscr{A}_{\alpha}(x, t) \text { for } x<0, \\
\left\|\mathrm{G}_{2}^{+}(x, t)\right\|_{L_{\xi}^{2}} \leq O(1) \mathscr{A}_{\alpha}(x, t) \text { for } x>0 .
\end{array}\right.
$$

By the standard bootstrap procedure in [13] for improving the $L_{\xi}^{2}$-estimate into $L_{\xi, 3}^{\infty}$, (5.11), and (5.12) together, one has

$$
\left\{\begin{array}{l}
\left\|\mathrm{G}_{2}(x, t)\right\|_{L_{\xi, 3}^{\infty}} \leq O(1) \mathscr{A}_{\alpha}(x, t) \text { for } x \in \mathbb{R} \\
\left\|\mathrm{G}_{2}^{-}(x, t)\right\|_{L_{\xi, 3}^{\infty}} \leq O(1) \mathscr{A}_{\alpha}(x, t) \text { for } x<0 \\
\left\|\mathrm{G}_{2}^{+}(x, t)\right\|_{L_{\xi, 3}^{\infty} \leq O(1) \mathscr{A}_{\alpha}(x, t) \text { for } x>0 .}
\end{array}\right.
$$

We continue to estimate $\left\|\mathrm{P}_{1}^{-} \mathrm{G}_{2}^{ \pm}\right\|_{L_{\xi}^{2}},\left\|\mathrm{P}_{1}^{-}\left(\mathrm{G}_{2}^{+}-\mathrm{G}_{2}^{-}\right)\right\|_{L_{\xi}^{2}},\left\|\mathrm{E}_{l}^{-}\left(\mathrm{G}_{2}^{+}-\mathrm{G}_{2}^{-}\right)\right\|_{L_{\xi}^{2}}$ for $l=$ $1,2,3$.

From (3.15) together with (A.9) (with $\omega_{0}=1 / 10$ in A.9), i.e. comparing the wave structure within the region $|x| \leq \varepsilon^{-\left(1+\omega_{0}\right)}$, (A.6), (A.7), and (A.8), we have that for $l=1,2$

$$
\begin{gathered}
\leq O(1) \int_{0}^{t} \int_{\mathbb{R}}\left(\varepsilon+\frac{1}{\sqrt{t-s+1}}\right)^{2}\left(\theta^{1}\left(x-z, t-s, \lambda_{3}^{-}, D_{0}\right)+\theta^{1}\left(x-z, t-s, \lambda_{3}^{+}, D_{0}\right)\right) \\
\cdot \frac{e^{-\frac{\varepsilon^{2} s}{E_{0}}-\frac{3 \varepsilon|z|}{D_{1}}}}{(1+s)^{\alpha / 2}} d z d s \\
+O(1) \int_{0}^{t} \int_{\mathbb{R}} \sum_{j=1}^{2} \min (\varepsilon \sqrt{t-s}, 1)\left(\theta^{1}\left(x-z, t-s, \lambda_{j}^{-}, D_{0}\right)+\theta^{1}\left(x-z, t-s, \lambda_{j}^{+}, D_{0}\right)\right) \\
\cdot \frac{e^{-\frac{\varepsilon^{2} s}{E_{0}}-\frac{3 \varepsilon|z|}{D_{1}}}}{(1+s)^{\alpha / 2}} d z d s \leq O(1) \frac{e^{-\frac{3 \varepsilon|x|}{D_{1}}-\frac{\varepsilon^{2} t}{E_{0}}}}{(1+t)^{\alpha / 2}}+O(1)\left\{\begin{array}{l}
\varepsilon^{9 / 20} \mathscr{A}_{\alpha}(x, t) \text { for }|x| \leq \varepsilon^{-11 / 10}, \\
\mathscr{A}_{\alpha}(x, t) \text { for }|x| \geq \varepsilon^{-11 / 10} .
\end{array}\right.
\end{gathered}
$$

From both (5.14) and the property that

$$
\left|\chi_{-}^{\prime}(x)\right| \leq O(1) \varepsilon e^{-\frac{4 \varepsilon|x|}{D_{0}}}
$$


one has that for $l=1,2$

$$
\left\|\chi_{-}^{\prime}(x) \mathrm{E}_{l}^{-, *}\left(\mathrm{G}_{2}^{+}(x, t)-\mathrm{G}_{2}^{-}(x, t)\right)\right\|_{L_{\xi}^{2}} \leq O(1) \varepsilon^{9 / 20} \frac{e^{-\frac{3 \varepsilon|x|}{D_{0}}-\frac{\varepsilon^{2} t}{E_{0}}}}{(1+t)^{\alpha / 2}} .
$$

Similarly to the argument for obtaining (5.14), by combining (3.15) and (A.10) together instead of (A.9),

$$
\begin{aligned}
& \left\|\mathrm{E}_{3}^{-, *}\left(\mathrm{G}_{2}^{+}(x, t)-\mathrm{G}_{2}^{-}(x, t)\right)\right\|_{L_{\xi}^{2}} \\
& \leq O(1) \int_{0}^{t} \int_{\mathbb{R}}\left(\varepsilon+\frac{1}{\sqrt{t-s+1}}\right) \\
& \cdot\left(\theta^{1}\left(x-z, t-s, \lambda_{3}^{-}, D_{0}\right)+\theta^{1}\left(x-z, t-s, \lambda_{3}^{+}, D_{0}\right)\right) \frac{e^{-\frac{\varepsilon^{2} s}{E_{0}}-\frac{3 \varepsilon|z|}{D_{1}}}}{(1+s)^{\alpha / 2}} d z d s \\
& +O(1) \int_{0}^{t} \int_{\mathbb{R}}\left(\varepsilon+\frac{1}{\sqrt{t-s+1}}\right) \\
& \cdot \sum_{j=1}^{2} \min (\varepsilon \sqrt{t-s}, 1)\left(\theta^{1}\left(x-z, t-s, \lambda_{j}^{-}, D_{0}\right)+\theta^{1}\left(x-z, t-s, \lambda_{j}^{+}, D_{0}\right)\right) \\
& \cdot \frac{e^{-\frac{\varepsilon^{2} s}{E_{0}}-\frac{3 \varepsilon|z|}{D_{1}}}}{(1+s)^{\alpha / 2}} d z d s \leq O(1) \varepsilon^{-1} \frac{e^{-\frac{3 \varepsilon|x|}{D_{1}}-\frac{\varepsilon^{2} t}{E_{0}}}}{(1+t)^{\alpha / 2}} \\
& +O(1)\left\{\begin{array}{r}
\varepsilon \sum_{j=1}^{2}\left(\mathbb{K}^{\alpha, 1 ; \varepsilon^{2} / E_{0}}\left(x, t ; \lambda_{j}^{-}, D_{0}, E_{0}\right)+\mathbb{K}^{\alpha, 1 ; \varepsilon^{2} / E_{0}}\left(x, t ; \lambda_{j}^{+}, D_{0}, E_{0}\right)\right) \\
\text { for }|x| \leq \varepsilon^{-11 / 10}, \\
\sum_{j=1}^{2}\left(\mathbb{K}^{\alpha, 2 ; \varepsilon^{2} / E_{0}}\left(x, t ; \lambda_{j}^{-}, D_{0}, E_{0}\right)+\mathbb{K}^{\alpha, 2 ; \varepsilon^{2} / E_{0}}\left(x, t ; \lambda_{j}^{+}, D_{0}, E_{0}\right)\right) \\
\text { for }|x| \geq \varepsilon^{-11 / 10} .
\end{array}\right.
\end{aligned}
$$

Here the function $\mathbb{K}^{\alpha, 2 ; \varepsilon^{2} / E_{0}}$ is given in (A.6). Then from this and (5.15) it follows that

$$
\left\|\chi_{-}^{\prime}(x) \mathrm{E}_{3}^{-, *}\left(\mathrm{G}_{2}^{+}(x, t)-\mathrm{G}_{2}^{-}(x, t)\right)\right\|_{L_{\xi}^{2}} \leq O(1) \frac{e^{-\frac{3 \varepsilon|x|}{D_{1}}-\frac{\varepsilon^{2} t}{E_{0}}}}{(1+t)^{\alpha / 2}} .
$$

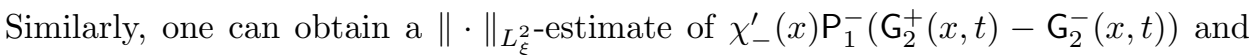
improve it into

$$
\left\|\chi_{-}^{\prime}(x) \mathrm{P}_{1}^{-}\left(\mathrm{G}_{2}^{+}(x, t)-\mathrm{G}_{2}^{-}(x, t)\right)\right\|_{L_{\xi, 3}^{\infty}} \leq O(1) \varepsilon \frac{e^{-\frac{3 \varepsilon|x|}{D_{1}}-\frac{\varepsilon^{2} t}{E_{0}}}}{(1+t)^{\alpha / 2}} .
$$

Next we continue to evaluate $\left(\mathrm{L}_{\varphi}-\mathrm{L}_{-}\right) \mathrm{G}_{2}^{-}(x, t)$. 
NONLINEAR WAVE PROPAGATIONS OVER A BOLTZMANN SHOCK PROFILE 1075

The estimates (5.16), (5.18), (5.19), and (5.13) together yield

$$
\chi_{+}\left\|\left(\mathrm{L}_{\varphi}-\mathrm{L}_{+}\right) \mathrm{G}_{2}^{+}\right\|_{L_{\xi, 2}^{\infty},} \chi_{-}\left\|\left(\mathrm{L}_{\varphi}-\mathrm{L}_{-}\right) \mathrm{G}_{2}^{-}\right\|_{L_{\xi, 2}^{\infty}} \leq O(1) \frac{e^{-\frac{3 \varepsilon|x|}{D_{1}}-\frac{\varepsilon^{2} t}{E_{0}}}}{(1+t)^{\alpha / 2}} \text { for } t \geq 0
$$

With the above estimates and the identity

$$
\begin{aligned}
\mathrm{ER}_{2}(x, t)=\chi_{+}(x)\left(\mathrm{L}_{\varphi}-\mathrm{L}_{+}\right) \mathrm{G}_{2}^{+}(x, t)+\chi_{-} & (x)\left(\mathrm{L}_{\varphi}-\mathrm{L}_{-}\right) \mathrm{G}_{2}^{-}(x, t) \\
& -\xi^{1} \chi_{-}^{\prime}(x)\left(\mathrm{G}_{2}^{-}(x, t)-\mathrm{G}_{2}^{+}(x, t)\right),
\end{aligned}
$$

one can have the estimate for $\mathrm{ER}_{2}$ with (5.16) and (5.19) together as follows.

For $l=1,2$ for $t \geq 0$

$$
\begin{aligned}
\left\|\mathrm{E}_{l}^{-, *} \mathrm{ER}_{2}\right\|_{L_{\xi}^{2}}= & \chi_{+}^{\prime}(x)\left\|\mathrm{E}_{l}^{-, *} \xi^{1}\left(\mathrm{G}_{2}^{+}-\mathrm{G}_{2}^{-}\right)\right\|_{L_{\xi}^{2}} \\
& \leq\left|\lambda_{l}^{-}\right| \chi_{+}^{\prime}(x)\left\|\mathrm{E}_{l}^{-, *}\left(\mathrm{G}_{2}^{+}-\mathrm{G}_{2}^{-}\right)\right\|_{L_{\xi}^{2}} \\
& +O(1) \chi_{+}^{\prime}(x)\left\|\mathrm{P}_{1}^{-}\left(\mathrm{G}_{2}^{+}-\mathrm{G}_{2}^{-}\right)\right\|_{L_{\xi}^{2}} \leq O(1) \varepsilon^{9 / 20} \frac{e^{-\frac{3 \varepsilon|x|}{D_{1}}-\frac{\varepsilon^{2} t}{E_{0}}}}{(1+t)^{\alpha / 2}} .
\end{aligned}
$$

With (5.17), (5.18), (5.19), one has

$$
\begin{array}{r}
\left\|\mathrm{E}_{3}^{-, *} \mathrm{ER}_{2}\right\|_{L_{\xi}^{2}} \leq O(1) \varepsilon \chi_{+}^{\prime}(x)\left\|\mathrm{E}_{3}^{-, *}\left(\mathrm{G}_{2}^{+}-\mathrm{G}_{2}^{-}\right)\right\|_{L_{\xi}^{2}}+O(1) \chi_{+}^{\prime}(x)\left\|\mathrm{P}_{1}^{-}\left(\mathrm{G}_{2}^{+}-\mathrm{G}_{2}^{-}\right)\right\|_{L_{\xi}^{2}} \\
\leq O(1) \varepsilon \frac{e^{-\frac{3 \varepsilon|x|}{D_{1}}-\frac{\varepsilon^{2} t}{E_{0}}}}{(1+t)^{\alpha / 2}} .
\end{array}
$$

The estimate (5.22) yields

$$
\left\|\mathrm{ER}_{2}^{t}\right\|_{L_{\xi}^{2}} \leq O(1) \varepsilon^{9 / 20} \frac{e^{-\frac{3 \varepsilon|x|}{D_{1}}-\frac{\varepsilon^{2} t}{E_{0}}}}{(1+t)^{\frac{\alpha}{2}}}
$$

and (5.23) gives

$$
\left\|\mathrm{ER}_{2}^{c}\right\|_{L_{\xi}^{2}} \leq O(1) \varepsilon \frac{e^{-\frac{3 \varepsilon|x|}{D_{1}}-\frac{\varepsilon^{2} t}{E_{0}}}}{(1+t)^{\frac{\alpha}{2}}}
$$


The factors $\chi_{-}(x)\left\|\mathrm{L}_{\varphi}-\mathrm{L}_{-}\right\|_{L_{\xi}^{2}}$ and $\chi_{+}(x)\left\|\mathrm{L}_{\varphi}-\mathrm{L}_{+}\right\|_{L_{\xi}^{2}}$ are bounded by $O(1) \varepsilon e^{-\frac{4 \varepsilon|x|}{D_{1}}}$. This and estimates in (5.13) together result in

$$
\begin{aligned}
\chi_{-}(x)\left\|\left(\mathrm{L}_{\varphi}-\mathrm{L}_{-}\right) \mathrm{G}_{2}^{-}\right\|_{L_{\xi}^{2}}, \quad \chi_{+}(x) \|\left(\mathrm{L}_{\varphi}-\mathrm{L}_{+}\right) & \mathrm{G}_{2}^{+} \|_{L_{\xi}^{2}} \\
& \leq O(1) \frac{e^{-\frac{3 \varepsilon|x|}{D_{1}}-\frac{\varepsilon^{2} t}{E_{0}}}}{(1+t)^{\alpha / 2}} \text { for } t \geq 0 .
\end{aligned}
$$

From (5.16), (5.18), (5.19), (5.26), and (5.21) together, one has a $\|\cdot\|_{L_{\xi}^{2} \text {-estimate }}$ improved into the $\|\cdot\|_{L_{\xi, 2}^{\infty}}$-estimate:

$$
\begin{aligned}
& \left\|\mathrm{ER}_{2}^{m}\right\|_{L_{\xi, 2}^{\infty}}=\left\|\mathrm{P}_{1}^{-} \mathrm{ER}_{2}\right\|_{L_{\xi, 2}^{\infty}} \\
& \leq O(1) \chi_{+}^{\prime}(x)\left(\sum_{j=1}^{3}\left\|\mathrm{E}_{j}^{-, *}\left(\mathrm{G}_{2}^{-}-\mathrm{G}_{2}^{+}\right)\right\|_{L_{\xi, 3}^{\infty}}+\left\|\mathrm{P}_{1}^{-}\left(\mathrm{G}_{2}^{-}-\mathrm{G}_{2}^{+}\right)\right\|_{L_{\xi, 3}^{\infty}}\right)
\end{aligned}
$$

$+\chi_{-}(x)\left\|\left(\mathrm{L}_{\varphi}-\mathrm{L}_{-}\right) \mathrm{G}_{2}^{-}\right\|_{L_{\xi, 2}^{\infty}}+\chi_{+}(x)\left\|\left(\mathrm{L}_{\varphi}-\mathrm{L}_{+}\right) \mathrm{G}_{2}^{+}\right\|_{L_{\xi, 2}^{\infty}} \leq O(1) \frac{e^{-\frac{3 \varepsilon|x|}{D_{1}}-\frac{\varepsilon^{2} t}{E_{0}}}}{(1+t)^{\alpha / 2}}$ for $t \geq 0$.

Hence, (5.22), (5.23), and (5.27) yield that for $t \geq 0$

$$
\left\{\begin{array}{l}
\left\|\mathrm{ER}_{2}^{t}\right\|_{L_{\xi}^{2}} \leq O(1) \varepsilon^{9 / 20} \frac{e^{-\frac{3 \varepsilon|x|}{D_{1}}-\frac{\varepsilon^{2} t}{E_{0}}}}{(1+t)^{\alpha / 2}}, \\
\left\|\mathrm{ER}_{2}^{c}\right\|_{L_{\xi}^{2}} \leq O(1) \varepsilon \frac{e^{-\frac{3 \varepsilon|x|}{D_{1}}-\frac{\varepsilon^{2} t}{E_{0}}}}{(1+t)^{\alpha / 2}}, \\
\left\|\mathrm{ER}_{2}^{m}\right\|_{L_{\xi, 2}^{\infty}} \leq O(1) \frac{e^{-\frac{3 \varepsilon|x|}{D_{1}}-\frac{\varepsilon^{2} t}{E_{0}}}}{(1+t)^{\alpha / 2}} .
\end{array}\right.
$$

The same argument in obtaining (5.28) yields (5.29) below as well:

$$
\left\{\begin{array}{l}
\sup _{x \in \mathbb{R}, t>0} \frac{\left\|\operatorname{ER}_{j}^{t}(x, t)\right\|_{L_{\xi}^{2}}}{\frac{e^{-\frac{3 \varepsilon|x|}{D_{1}}-\frac{\varepsilon^{2} t}{E_{0}}}}{(1+t)^{\alpha / 2}}} \leq\left(C_{2} \varepsilon\right)^{9(j-1) / 20}, \\
\sup _{x \in \mathbb{R}, t>0} \frac{\left\|\operatorname{ER}_{j}^{c}(x, t)\right\|_{L_{\xi}^{2}}}{\frac{e^{-\frac{3 \varepsilon|x|}{D_{1}}-\frac{\varepsilon^{2} t}{E_{0}}}}{(1+t)^{\alpha / 2}}} \leq \varepsilon\left(C_{2} \varepsilon\right)^{9(j-2) / 20}, \\
\sup _{x \in \mathbb{R}, t>0} \frac{\left\|\operatorname{ER}_{j}^{m}(x, t)\right\|_{L_{\xi, 2}^{\infty}}}{\frac{e^{-\frac{3 \varepsilon|x|}{D_{1}}-\frac{\varepsilon^{2} t}{E_{0}}}}{(1+t)^{\alpha / 2}}} \leq C_{2}\left(C_{2} \varepsilon\right)^{9(j-2) / 20},
\end{array}\right.
$$

for some constant $C_{2}>0$ independent of $\varepsilon$. This results in the convergence of $\sum_{j=1}^{\infty}\left\|\mathrm{ER}^{t}\right\|_{L_{\xi, 3}^{\infty}}^{\infty}$ as well as

$$
\left\|\sum_{j=2}^{\infty} \mathrm{G}_{j}(x, t)\right\|_{L_{\xi, 3}^{\infty}}=O(1)\left\|\mathrm{G}_{2}(x, t)\right\|_{L_{\xi, 3}^{\infty}},
$$

and the estimates in (5.13) conclude (5.6). One concludes (5.7) and (5.8) from (5.29). The lemma follows. 
Similarly, one has the following lemma.

Lemma 5.2. For the function $\tilde{\mathscr{I}}_{\alpha}$ defined in (5.3) with $\alpha \in[1,2]$, the function $\mathbb{T}\left[\tilde{\mathscr{I}}_{\alpha}\right](x, t)$ defined in (4.34) satisfies

$$
\begin{aligned}
& \left\|\mathbb{T}\left[\tilde{\mathscr{I}}_{\alpha}\right](x, t)\right\|_{L_{\xi, 3}^{\infty}} \leq O(1) \mathscr{B}_{\alpha}(x, t), \\
& \left|\left(\mathrm{I}_{3}, \tilde{\mathscr{I}}_{\alpha}-\left(\partial_{t}+\xi^{1} \partial_{x}-\mathrm{L}_{\varphi}\right) \mathbb{T}\left[\tilde{\mathscr{I}}_{\alpha}\right]\right)\right| \leq O(1) \frac{e^{-\frac{3 \varepsilon|x|}{D_{1}}}\left(\varepsilon e^{-\frac{\varepsilon t}{E_{0}}}+\varepsilon^{\frac{3}{2}} e^{-\frac{\varepsilon^{2} t}{E_{0}}}\right)}{(1+t)^{\frac{\alpha}{2}}}, \\
& \left\|\left(\tilde{\mathscr{I}}_{\alpha}-\left(\partial_{t}+\xi^{1} \partial_{x}-\mathrm{L}_{\varphi}\right) \mathbb{T}\left[\tilde{\mathscr{I}}_{\alpha}\right]\right)^{m}\right\|_{L_{\xi, 2}^{\infty}} \\
& \leq O(1) \frac{e^{-\frac{3 \varepsilon|x|}{D_{1}}}\left(e^{-\frac{\varepsilon t}{E_{0}}}+\varepsilon^{\frac{1}{2}} e^{-\frac{\varepsilon^{2} t}{E_{0}}}\right)}{(1+t)^{\frac{\alpha}{2}}},
\end{aligned}
$$

where the function $\mathscr{B}_{\alpha}(x, t)$ is

$$
\begin{aligned}
& \text { (5.34) } \mathscr{B}_{\alpha}(x, t) \equiv \\
& \left\{\begin{array}{c}
\varepsilon^{-1} \frac{e^{-\frac{3 \varepsilon|x|}{D_{1}}}\left(e^{-\frac{\varepsilon t}{E_{0}}}+\varepsilon^{\frac{1}{2}} e^{-\frac{\varepsilon^{2} t}{E_{0}}}\right)}{(t+1)^{\frac{\alpha}{2}}} \text { for } x>0, \\
\sum_{j=1}^{2}\left(\frac{\sqrt{|x|+1}}{\left(x-\lambda_{j}^{-} t\right)^{\frac{\alpha}{2}}(1+\varepsilon \sqrt{x+1})}+\frac{\varepsilon^{-1} e^{-C \varepsilon x}}{(t+1)^{\frac{\alpha}{2}}}+\Gamma^{\alpha}\left(\varepsilon^{-1}\right) e^{-C \varepsilon\left|x-\lambda_{j}^{-} t\right|}\right) \\
\cdot\left(e^{-\frac{\varepsilon^{2}\left|x-\lambda_{j}^{-} t\right|}{\lambda_{j}^{-} E}}+\varepsilon^{\frac{1}{2}} e^{-\frac{\varepsilon\left|x-\lambda_{j}^{-} t\right|}{\lambda_{j}^{-} E_{0}}}\right) \chi_{\left[\lambda_{j}^{-}(t+1)+\sqrt{t+1}, 0\right]}(x) \\
+\left(\Gamma^{\alpha}(t+1) e^{-C \varepsilon t}+\Gamma^{\alpha}(\sqrt{t+1})(1+\varepsilon \sqrt{t})^{-1}+\frac{\varepsilon^{-1} e^{-C \varepsilon t}}{(t+1)^{\frac{\alpha}{2}}}\right) \\
\cdot \chi_{\left[-\sqrt{t+1}+\lambda_{j}^{-}(t+1), \sqrt{t+1}+\lambda_{j}^{-}(t+1)\right]}(x)+\left[\frac{\Gamma^{\alpha}(\sqrt{t+1}) e^{-\frac{\left(x-\lambda_{j}^{-} t\right)^{2}}{D_{1}(t+1)}}}{(1+\varepsilon \sqrt{t})}+\frac{(t+1)^{\frac{1}{2}} e^{-C \varepsilon\left|x-\lambda_{j}^{-} t\right|}}{\left(x-\lambda_{j}^{-} t\right)^{\frac{\alpha}{2}}}\right. \\
\left.+\Gamma^{\alpha}\left(\varepsilon^{-1}\right) e^{-C \varepsilon\left|x-\lambda_{j}^{-} t\right|} e^{-C \varepsilon^{2} t}+\varepsilon^{\frac{\alpha-2}{2}} e^{-\frac{3 \varepsilon\left|x-\lambda_{j}^{-} t\right|}{D_{1}}}\right] \chi_{\left[-\infty, \lambda_{j}^{-}(t+1)-\sqrt{t+1]}(x)\right.}(x) \\
+\left[\frac{e^{-C \varepsilon\left(\lambda_{j}^{-} t-x\right)}}{\left(x-\lambda_{j}^{-} t\right)^{\frac{\alpha-1}{2}}}+\frac{\varepsilon^{-1} e^{-C \varepsilon t} e^{-\frac{3 \varepsilon\left(\left|x-\lambda_{j}^{-} t\right|\right)}{D_{1}}}}{(t+1)^{\frac{\alpha}{2}}}\right] \chi_{\left[-\infty, \lambda_{j}^{-}(t+1)-\sqrt{t+1]}\right.}(x) \text { for } x<0 .
\end{array}\right.
\end{aligned}
$$

\subsection{Estimates on local wave front tracing.}

Lemma 5.3. For the functions $\mathscr{I}_{\alpha}$ and $\tilde{\mathscr{I}}_{\alpha}$ given in (5.2) and (5.3), the operators $\mathbb{D}$ and $\mathbb{R}_{3}$ given in (4.37) and (4.38), respectively, satisfy

$$
\begin{array}{r}
\mathbb{D}\left[\mathscr{I}_{\alpha}\right](x, t)=d(t) \varphi^{\prime}(x) \text { with } d^{\prime}(t)=O(1) \varepsilon^{-21 / 20} \frac{e^{-\frac{\varepsilon^{2} t}{E_{0}}}}{(1+t)^{\alpha / 2}}, \\
\mathbb{D}\left[\tilde{\mathscr{I}}_{\alpha}\right](x, t)=d_{1}(t) \varphi^{\prime}(x) \text { with } d_{1}^{\prime}(t)=O(1) \varepsilon^{-21 / 20} \frac{\left(e^{-\frac{\varepsilon t}{E_{0}}}+\varepsilon^{\frac{1}{2}} e^{-\frac{\varepsilon^{2} t}{E_{0}}}\right)}{(1+t)^{\alpha / 2}},
\end{array}
$$




$$
\begin{aligned}
& \left\{\begin{array}{l}
\left|\left(\mathrm{I}_{j}, \mathbb{R}_{3}\left[\mathscr{I}_{\alpha}\right]\right)\right| \leq O(1) \varepsilon^{39 / 20} \frac{e^{-\frac{\varepsilon^{2} t}{E_{0}}}}{(1+t)^{\alpha / 2}} e^{-\frac{3 \varepsilon|x|}{D_{1}}} \text { for } j=1,2, \\
\left|\left(\mathrm{I}_{3}, \mathbb{R}_{3}\left[\mathscr{I}_{\alpha}\right]\right)\right| \leq O(1) \varepsilon^{19 / 20} \frac{e^{-\frac{\varepsilon^{2} t}{E_{0}}}}{(1+t)^{\alpha / 2}} e^{-\frac{3 \varepsilon|x|}{D_{1}}},
\end{array}\right. \\
& \left\{\begin{array}{l}
\left|\left(\mathrm{I}_{j}, \mathbb{R}_{3}\left[\tilde{\mathscr{I}}_{\alpha}\right]\right)\right| \leq O(1) \varepsilon^{39 / 20} \frac{\left(e^{-\frac{\varepsilon t}{E_{0}}}+\varepsilon^{\frac{1}{2}} e^{-\frac{\varepsilon^{2} t}{E_{0}}}\right)}{(1+t)^{\alpha / 2}} e^{-\frac{3 \varepsilon|x|}{D_{1}}} \text { for } j=1,2, \\
\left|\left(\mathrm{I}_{3}, \mathbb{R}_{3}\left[\tilde{\mathscr{I}}_{\alpha}\right]\right)\right| \leq O(1) \varepsilon^{19 / 20} \frac{\left(e^{-\frac{\varepsilon t}{E_{0}}}+\varepsilon^{\frac{1}{2}} e^{-\frac{\varepsilon^{2} t}{E_{0}}}\right)}{(1+t)^{\alpha / 2}} e^{-\frac{3 \varepsilon|x|}{D_{1}}},
\end{array}\right. \\
& \left\|\mathrm{P}_{1}^{-} \mathbb{R}_{3}\left[\mathscr{I}_{\alpha}\right]\right\|_{L_{\xi}^{2}} \leq O(1) \frac{e^{-\frac{\varepsilon^{2} t}{E_{0}}}}{(1+t)^{\alpha / 2}} e^{-\frac{\varepsilon|x|}{D_{1}}} \\
& \left\|\mathrm{P}_{1}^{-} \mathbb{R}_{3}\left[\tilde{\mathscr{I}}_{\alpha}\right]\right\|_{L_{\xi, 2}^{\infty}} \leq O(1) \frac{\left(e^{-\frac{\varepsilon t}{E_{0}}}+\varepsilon^{\frac{1}{2}} e^{-\frac{\varepsilon^{2} t}{E_{0}}}\right)}{(1+t)^{\alpha / 2}} e^{-\frac{3 \varepsilon|x|}{D_{1}}} .
\end{aligned}
$$

Proof. One relaxes the factor $\varepsilon$ in (5.7) into a weaker upper bound $\varepsilon^{19 / 20}$ so that it follows that

$$
\left\|\sum_{j=2}^{\infty} \operatorname{ER}_{j}^{c}(x, t)\right\|_{L_{\xi}^{2}}=O(1) \varepsilon^{19 / 20} \frac{e^{-\frac{\varepsilon^{2} t}{E_{0}}-\frac{3 \varepsilon|x|}{D_{1}}}}{(1+t)^{\alpha / 2}} .
$$

This and the definition (4.37) yield (5.35), (5.37). From (5.8), it follows that

$$
\left\|\sum_{j=2}^{\infty} \operatorname{ER}_{j}^{m}(x, t)\right\|_{L_{\xi, 2}^{\infty}}=O(1) \frac{e^{-\frac{\varepsilon^{2} t}{E_{0}}-\frac{3 \varepsilon|x|}{D_{1}}}}{(1+t)^{\alpha / 2}} .
$$

The above concludes (5.39), and the part for the function $\tilde{\mathscr{I}}_{\alpha}$ will follow by the same argument. The lemma follows.

5.3. Energy estimates. The defining functions $\mathrm{C}(x, t) \equiv \mathbb{C}_{3}\left[\mathbb{R}_{3}\left[\mathscr{I}_{\alpha}\right]\right](x, t)$ and $\tilde{\mathrm{C}}(x, t) \equiv \mathbb{C}_{3}\left[\tilde{\mathbb{R}}_{3}\left[\mathscr{I}_{\alpha}\right]\right](x, t)$ of the compressive operators are defined by the initial value problems:

$$
\left\{\begin{array}{l}
\partial_{t} \mathrm{C}+\xi^{1} \partial_{x} \mathrm{C}-\mathrm{L}_{\varphi} \mathrm{C}+\varepsilon \sum_{j=1}^{2}\left(\mathrm{I}_{j}, \mathrm{C}\right) \mathrm{r}_{j}=\overline{\mathrm{S}}(x, t), \\
\partial_{t} \tilde{\mathrm{C}}+\xi^{1} \partial_{x} \tilde{\mathrm{C}}-\mathrm{L}_{\varphi} \tilde{\mathrm{C}}+\varepsilon \sum_{j=1}^{2}\left(\mathrm{I}_{j}, \tilde{\mathrm{C}}\right) \mathrm{r}_{j}=\tilde{\mathrm{S}}(x, t), \\
\mathrm{C}(x, 0), \tilde{\mathrm{C}}(x, 0) \equiv 0,
\end{array}\right.
$$

where

$$
\left\{\begin{array}{l}
\bar{S} \equiv \mathbb{R}_{3}\left[\mathscr{I}_{\alpha}\right] \\
\tilde{S} \equiv \mathbb{R}_{3}\left[\tilde{\mathscr{I}}_{\alpha}\right]
\end{array}\right.
$$


Lemma 5.3 yields the structures of $\overline{\mathrm{S}}$ and $\tilde{\mathrm{S}}$ :

$$
\begin{aligned}
& \left\{\begin{array}{l}
\left|\left(\mathrm{I}_{j}, \overline{\mathrm{S}}(x, t)\right)\right| \leq O(1) \varepsilon^{39 / 20} \frac{e^{-\frac{\varepsilon^{2} t}{E_{0}}}}{(1+t)^{\alpha / 2}} e^{-3 \varepsilon|x| / D_{1}} \text { for } j=1,2, \\
\left|\left(\mathrm{I}_{j}, \tilde{\mathrm{S}}(x, t)\right)\right| \leq O(1) \varepsilon^{39 / 20} \frac{\left(e^{-\frac{\varepsilon t}{E_{0}}}+\varepsilon^{\frac{1}{2}} e^{-\frac{\varepsilon^{2} t}{E_{0}}}\right)}{(1+t)^{\alpha / 2}} e^{-3 \varepsilon|x| / D_{1}} \text { for } j=1,2, \\
\left|\left(\mathrm{I}_{3}, \overline{\mathrm{S}}(x, t)\right)\right| \leq O(1) \varepsilon^{19 / 20} \frac{e^{-\frac{\varepsilon^{2} t}{E_{0}}}}{(1+t)^{\alpha / 2}} e^{-3 \varepsilon|x| / D_{1}}, \\
\left|\left(I_{3}, \tilde{\mathrm{S}}(x, t)\right)\right| \leq O(1) \varepsilon^{19 / 20} \frac{\left(e^{-\frac{\varepsilon t}{E_{0}}}+\varepsilon^{\frac{1}{2}} e^{-\frac{\varepsilon^{2} t}{E_{0}}}\right)}{(1+t)^{\alpha / 2}} e^{-3 \varepsilon|x| / D_{1}}
\end{array}\right. \\
& \left\{\begin{array}{l}
\left\|\mathrm{P}_{1}^{-} \overline{\mathrm{S}}(x, t)\right\|_{L_{\xi, 2}^{\infty}} \leq O(1) \frac{e^{-\frac{\varepsilon^{2} t}{E_{0}}}}{(1+t)^{\alpha / 2}} e^{-3 \varepsilon|x| / D_{1}}, \\
\left\|\mathrm{P}_{1}^{-} \tilde{\mathrm{S}}(x, t)\right\|_{L_{\xi, 2}^{\infty}} \leq O(1) \frac{\left(e^{-\frac{\varepsilon t}{E_{0}}}+\varepsilon^{\frac{1}{2}} e^{-\frac{\varepsilon^{2} t}{E_{0}}}\right)}{(1+t)^{\alpha / 2}} e^{-3 \varepsilon|x| / D_{1}},
\end{array}\right. \\
& \int_{\mathbb{R}} \mathrm{P}_{0}^{-} \overline{\mathrm{S}}(x, t) d x \equiv 0, \int_{\mathbb{R}} \mathrm{P}_{0}^{-} \tilde{\mathrm{S}}(x, t) d x \equiv 0 .
\end{aligned}
$$

Without repeating the same analysis for $\mathrm{C}(x, t)$ and $\tilde{\mathrm{C}}(x, t)$, we show the energy estimates for the function $\mathrm{C}(x, t)$ only. From (5.43) one has

$$
\begin{aligned}
& \frac{d}{d t} \int_{\mathbb{R}}\left(\mathrm{I}_{k}, \mathrm{C}\right) d x \\
& +\int_{\mathbb{R}}\left(\mathrm{I}_{k}, \xi^{1} \partial_{x} \mathrm{C}-\mathrm{L}_{\varphi} \mathrm{C}+\varepsilon \sum_{j=1}^{2}\left(\mathrm{I}_{j}, \mathrm{C}\right) \mathrm{r}_{j}-\overline{\mathrm{S}}(x, t)\right) d x=0 \text { for } k=1,2,3
\end{aligned}
$$

From (5.46) and (5.47) together, one has

$$
\left\{\begin{array}{l}
\frac{d}{d t} \int_{\mathbb{R}}\left(\mathrm{I}_{k}, \mathrm{C}\right) d x+\varepsilon \int_{\mathbb{R}}\left(\mathrm{I}_{k}, \mathrm{C}\right) d x=0, \quad k=1,2, \\
\frac{d}{d t} \int_{\mathbb{R}}\left(\mathrm{I}_{3}, \mathrm{C}\right) d x=0 .
\end{array}\right.
$$

From the zero initial data property,

$$
\int_{\mathbb{R}}\left(\mathrm{I}_{k}, \mathrm{C}(x, 0)\right) d x=0 \text { for } k=1,2,3 .
$$

This and (5.48) give rise to the macroscopic conservation laws for $\mathrm{C}(x, t)$ :

$$
\int_{\mathbb{R}} \mathrm{P}_{0}^{-} \mathrm{C}(x, t) d x=0 \text { for } t \geq 0 .
$$

Remark 5.4. The rest of this subsection is a variant of the macro-micro decomposition and energy estimates in [12, which is implemented from the viscous shock wave stability in 2 . With the presence of the upwind damping, an exponentially growing weighted function is introduced in the energy estimates to yield a global exponential decaying rate in time variable. The order of the small parameter $\varepsilon$ is important for showing the convergence of the $\mathbb{T}-\mathbb{C}$ scheme. The estimate is a 
modification of the lower energy estimate of [12. However, we will need to show the estimates in order to obtain the exponential decaying rate of convergence in terms of $\varepsilon$.

We use the anti-derivative variable in order to effectively make use of the conservative property (5.49):

$$
\mathrm{W}(x, t) \equiv \int_{-\infty}^{x} \mathrm{C}(z, t) d z
$$

The equation for $\mathrm{W}(x, t)$ is

$$
\left\{\begin{array}{l}
\partial_{t} \mathrm{~W}+\xi^{1} \partial_{x} \mathrm{~W}-\int_{-\infty}^{x} \mathrm{~L}_{\varphi} \mathrm{C}(z, t) d y+\varepsilon \sum_{j=1}^{2}\left(\mathrm{I}_{j}, \mathrm{~W}\right) \mathrm{I}_{j}=\check{\boldsymbol{S}}(x, t), \\
\mathrm{W}(x, 0) \equiv 0
\end{array}\right.
$$

where

$$
\check{\boldsymbol{S}}(x, t)=\int_{-\infty}^{x} \overline{\mathbf{S}}(z, t) d z .
$$

From (5.44), (5.45), and (5.46), the function $\check{\boldsymbol{S}}(x, t)$ satisfies

$$
\left\{\begin{array}{l}
\left|\left(\mathrm{I}_{j}, \check{\boldsymbol{S}}\right)\right| \leq \varepsilon^{19 / 20} \frac{e^{-\varepsilon^{2} t / E_{0}} e^{-3 \varepsilon|x| / D_{1}}}{(1+t)^{\alpha / 2}} \text { for } j=1,2, \\
\left|\left(\mathrm{I}_{3}, \check{\boldsymbol{S}}\right)\right| \leq \varepsilon^{-1 / 20} \frac{e^{-\varepsilon^{2} t / E_{0}} e^{-3 \varepsilon|x| / D_{1}}}{(1+t)^{\alpha / 2}}, \\
\int_{\mathbb{R}}\left\|\mathrm{P}_{0}^{-\check{\boldsymbol{S}}}\right\|_{L_{\xi}^{2}}^{2} e^{3 \varepsilon|x| / D_{1}} d x \leq O(1) \varepsilon^{-22 / 20} \frac{e^{-2 \varepsilon^{2} t / E_{0}}}{(1+t)^{\alpha}} .
\end{array}\right.
$$

By [1], the Boltzmann shock profile $\varphi$ is close to the local Maxwellian profile $\psi_{N S}(x) \equiv \mathrm{M}_{[\bar{\rho}, \bar{u}, \overline{\boldsymbol{T}}]}$ in the sense of (2.13) where $[\bar{\rho}, \bar{u}, \overline{\boldsymbol{T}}]$ is given in (2.14) with $s=0$. With the velocity profile $\bar{u}(x)$ and the temperature profile $\overline{\boldsymbol{T}}(x)$, we consider the following local macro-micro decomposition with respect to the local Maxwellian $\mathrm{M}_{[1, \bar{u}(x), \overline{\boldsymbol{T}}(x)]}$ for the purpose of obtaining energy estimates with exponential rate decaying in the time variable:

$$
\left\{\begin{array}{l}
\mathscr{P}_{0} \mathrm{~g}(x, t) \equiv \sum_{j=1}^{3}\left(\tilde{\mathrm{E}}_{j} \frac{\mathrm{M}_{-}}{\mathrm{M}_{[1, \bar{u}(x), \overline{\boldsymbol{T}}(x)]}}, \mathrm{g}(x, t)\right) \tilde{\mathrm{E}}_{j}, \\
\mathscr{P}_{1} \mathrm{~g}(x, t)=\mathrm{g}(x, t)-\mathscr{P}_{0} \mathrm{~g}(x, t)
\end{array}\right.
$$

where

$$
\left\{\begin{array}{l}
\tilde{\mathrm{E}}_{1} \equiv\left(-\frac{\left(\xi^{1}-\bar{u}(x)\right)}{\sqrt{2 \overline{\boldsymbol{T}}(x)}}+\frac{\left|\xi^{1}-\bar{u}(x)\right|^{2}+\sum_{j=2}^{3}\left|\xi^{j}\right|^{2}}{\sqrt{30} \overline{\boldsymbol{T}}(x)}\right) \frac{\mathrm{M}_{[1, \bar{u}(x), \overline{\boldsymbol{T}}(x)]}}{\mathrm{M}_{-}^{\frac{1}{2}}} \\
\tilde{\mathrm{E}}_{2} \equiv\left(-\sqrt{\frac{5}{2}}+\frac{1}{\sqrt{10}} \frac{\left|\xi^{1}-\bar{u}(x)\right|^{2}+\sum_{j=2}^{3}\left|\xi^{j}\right|^{2}}{\overline{\boldsymbol{T}}(x)}\right) \frac{\mathrm{M}_{[1, \bar{u}(x), \overline{\boldsymbol{T}}(x)]}}{\mathrm{M}_{-}^{\frac{1}{2}}} \\
\tilde{\mathrm{E}}_{3} \equiv\left(\frac{\left(\xi^{1}-\bar{u}(x)\right)}{\sqrt{2 \overline{\boldsymbol{T}}(x)}}+\frac{\left|\xi^{1}-\bar{u}(x)\right|^{2}+\sum_{j=2}^{3}\left|\xi^{j}\right|^{2}}{\sqrt{30} \overline{\boldsymbol{T}}(x)}\right) \frac{\mathrm{M}_{[1, \bar{u}(x), \overline{\boldsymbol{T}}(x)]}}{\mathrm{M}_{-}^{\frac{1}{2}}}
\end{array}\right.
$$

Remark 5.5. The energy estimates in the rest of this subsection are modified from those in [12, and the estimates are strong enough to obtain the global exponential decaying rate. The anti-derivative variables and energy methods for viscous conservation laws were independently introduced in [2, 18]. 
We denote the macroscopic and microscopic components referring to the decomposition (5.52) by

$$
\mathrm{W}_{0}(x, t) \equiv \mathscr{P}_{0} \mathrm{~W}, \quad \mathrm{~W}_{1} \equiv \mathscr{P}_{1} \mathrm{~W} .
$$

Then one applies this macro-micro decomposition to (5.50) to yield that

$$
\partial_{t} \mathrm{~W}_{0}+\mathscr{P}_{0} \xi^{1} \mathscr{P}_{0} \partial_{x} \mathrm{~W}_{0}+\mathscr{P}_{0} \xi^{1} \mathscr{P}_{1} \mathrm{C}+\varepsilon \sum_{j=1}^{2}\left(\mathrm{l}_{j}, \mathrm{~W}_{0}\right) \mathscr{P}_{0} \mathrm{r}_{j}=\mathscr{P}_{0} \check{\boldsymbol{S}}
$$

From the property

$$
\mathscr{P}_{0} \partial_{x} \mathrm{~W}_{0}=\mathscr{P}_{0} \mathrm{C}
$$

and (5.43) together, one has

$$
\begin{aligned}
& \mathscr{P}_{1} \mathrm{C} \\
& =\mathrm{L}_{\psi_{N S}}^{-1}\left[\partial_{t} \mathscr{P}_{1} \mathrm{C}+\mathscr{P}_{1} \xi^{1} \partial_{x}\left(\mathscr{P}_{0} \partial_{x} \mathrm{~W}_{0}+\mathscr{P}_{1} \mathrm{C}\right)-\left(\mathrm{L}_{\varphi}-\mathrm{L}_{\psi_{N S}}\right) \mathrm{C}-\overline{\mathrm{S}}(x, t)\right] .
\end{aligned}
$$

Here the operator $L_{\psi_{N S}}^{-1}$ is well-defined on the range of microscopic components. By substituting (5.55) and (5.56) into (5.54) one has

$$
\begin{aligned}
\partial_{t} \mathrm{~W}_{0}+\mathscr{P}_{0} \xi^{1} \mathscr{P}_{0} \partial_{x} \mathrm{~W}_{0}+\mathscr{P}_{0} \xi^{1} \mathrm{~L}_{\psi_{N S}}^{-1}\left[\mathscr{P}_{1} \xi^{1} \partial_{x} \mathscr{P}_{0} \partial_{x} \mathrm{~W}_{0}\right] \\
+\varepsilon \sum_{j=1}^{2}\left(\mathrm{I}_{j}, \mathrm{~W}_{0}\right) \mathscr{P}_{0} \mathrm{r}_{j} \\
=-\mathscr{P}_{0} \xi^{1} \mathrm{~L}_{\psi_{N S}}^{-1}\left[\partial_{t} \mathscr{P}_{1} \mathrm{C}+\partial_{x} \xi^{1} \mathscr{P}_{1} \mathrm{C}-\left(\mathrm{L}_{\varphi}-\mathrm{L}_{\psi_{N S}}\right) \mathrm{C}-\overline{\mathrm{S}}(x, t)\right]+\mathscr{P}_{0} \check{\boldsymbol{S}} .
\end{aligned}
$$

One decomposes the local macroscopic component $\mathbf{W}_{0}$ in terms of $\tilde{\mathbf{E}}_{j}, j=1,2,3$ :

$$
\mathrm{W}_{0}(x, t)=\sum_{j=1}^{3} W_{0}^{j}(x, t) \tilde{\mathrm{E}}_{j}(x, t)
$$

and it follows that

$$
\begin{aligned}
& \left(\mathrm{W}_{0}, \xi^{1} \mathbf{W}_{0}\right)=\sum_{i=1}^{3} \lambda_{i}\left|W_{0}^{i}\right|^{2}, \\
& \left\{\begin{array}{l}
\lambda_{1}(x)=\bar{u}(x)-\sqrt{5 \overline{\boldsymbol{T}}(x) / 3}, \\
\lambda_{2}(x)=\bar{u}(x), \\
\lambda_{3}(x)=\bar{u}(x)+\sqrt{5 \overline{\boldsymbol{T}}(x) / 3}=O(1) \varepsilon .
\end{array}\right.
\end{aligned}
$$

Take the $L_{\xi}^{2}$-inner product of (5.57) with $\mathrm{W}_{0}(x, t)$ together to yield that

$$
\begin{aligned}
& \sum_{j=1}^{3}\left(W_{0}^{j} \partial_{t} W_{0}^{j}+W_{0}^{j} \lambda_{j} \partial_{x} W_{0}^{j}+O(1) \varepsilon^{2}\left|W_{0}^{j}\right|^{2}\left|\lambda_{j}\right| e^{-\varepsilon|x| / D_{1}}\right) \\
& +\left(\mathrm{W}_{0}, \mathscr{P}_{0} \xi^{1} \mathrm{~L}_{\psi_{N S}}^{-1}\left[\mathscr{P}_{1} \xi^{1} \partial_{x} \mathscr{P}_{0} \partial_{x} \mathrm{~W}_{0}\right]\right) \\
& +\sum_{l=1}^{2}\left(\varepsilon+O(1) \varepsilon^{2}\right)\left|W_{0}^{l}\right|^{2}+O(1) \varepsilon^{3}\left|W_{0}^{3}\right|^{2} \\
& +\left(\mathrm{W}_{0}, \mathscr{P}_{0} \xi^{1} \mathrm{~L}_{\psi_{N S}}^{-1}\left[\partial_{t} \mathscr{P}_{1} \mathrm{C}+\partial_{x} \xi^{1} \mathscr{P}_{1} \mathrm{C}-\left(\mathrm{L}_{\varphi}-\mathrm{L}_{\psi_{N S}}\right) \mathrm{C}-\overline{\mathrm{S}}(x, t)\right]\right)-\left(\mathrm{W}_{0}, \mathscr{P}_{0} \check{\boldsymbol{S}}\right)=0 .
\end{aligned}
$$


Proposition 5.6. There exists $\nu_{1} \gg 1 / D_{1}$ such that the solutions $\mathrm{W}_{0}$ of (5.54) and $\mathrm{C}(x, t)$ of (5.43) satisfy

$$
\begin{aligned}
& \frac{d}{d t} \int_{\mathbb{R}} \cosh \left(\varepsilon x / D_{1}\right)\left(\frac{\left\|\mathrm{W}_{0}\right\|_{L_{\xi}^{2}}^{2}}{2}+\left(\xi^{1} \mathrm{~W}_{0}, \mathrm{~L}_{\psi_{N S}}^{-1} \mathscr{P}_{1} \mathrm{C}\right)\right) d x \\
& +\int_{\mathbb{R}} \cosh \left(\varepsilon x / D_{1}\right)\left(\frac{\varepsilon^{2}\left\|\mathrm{~W}_{0}\right\|_{L_{\xi}^{2}}^{2}}{8 A_{1} D_{1}^{2}}+\frac{\nu_{1}\left\|\mathscr{P}_{1} \xi^{1} \partial_{x} \mathrm{~W}_{0}\right\|_{L_{\xi}^{2}}^{2}}{2}\right) d x \\
& \leq O(1)\left\{\int_{\mathbb{R}} \cosh \left(\varepsilon x / D_{1}\right)\left\|\mathscr{P}_{1} \mathrm{C}\right\|_{L_{\xi}^{2}}^{2} d x+\frac{\varepsilon^{-62 / 20}}{(1+t)^{\alpha}} e^{-\frac{2 \varepsilon^{2} t}{E_{0}}}\right\}
\end{aligned}
$$

Proof. By multiplying (5.59) with a weighted function $\cosh \left(\varepsilon x / D_{1}\right)$, one integrates it over $\mathbb{R}$ to yield that

$$
\begin{aligned}
& 0=\frac{d}{d t} \int_{\mathbb{R}} \cosh \left(\varepsilon x / D_{1}\right) \frac{1}{2} \sum_{j=1}^{3}\left|W_{0}^{j}\right|^{2} d x \\
& +\int_{\mathbb{R}} \cosh \left(\varepsilon x / D_{1}\right) \sum_{j=1}^{2}\left[-\left(\partial_{x} \lambda_{j}\right)-\frac{\varepsilon \lambda_{j} \tanh \left(\varepsilon x / D_{1}\right)}{D_{1}}\right] \frac{\left|W_{0}^{j}\right|^{2}}{2} d x\left(\equiv I_{1}\right) \\
& +\int_{\mathbb{R}} \cosh \left(\varepsilon x / D_{1}\right)\left(\sum_{l=1}^{2}\left(\varepsilon+O(1) \varepsilon^{2}\right)\left|W_{0}^{l}\right|^{2}+O(1) \varepsilon^{3}\left|W_{0}^{3}\right|^{2}\right) d x\left(\equiv I_{2}\right) \\
& +\int_{\mathbb{R}} \cosh \left(\varepsilon x / D_{1}\right)\left(\left[-\left(\partial_{x} \lambda_{3}\right)-\frac{\varepsilon \lambda_{3} \tanh \left(\varepsilon x / D_{1}\right)}{2 D_{1}}\right] \frac{\left|W_{0}^{3}\right|^{2}}{2}\right. \\
& -\int_{\mathbb{R}} \cosh \left(\varepsilon x / D_{1}\right)\left(\mathscr{P}_{1} \xi^{1} \mathrm{~W}_{0}, \frac{\varepsilon \tanh \left(\varepsilon x / D_{1}\right)}{D_{1}} \mathrm{~L}_{\psi_{N S}}^{-1}\left[\mathscr{P}_{1} \xi^{1} \mathscr{P}_{0} \partial_{x} \mathrm{~W}_{0}\right]\right) d x\left(\equiv I_{4}\right) \\
& -\int_{\mathbb{R}} \cosh \left(\varepsilon x / D_{1}\right)\left(\mathscr{P}_{1} \xi^{1} \mathscr{P}_{0} \partial_{x} \mathrm{~W}_{0}, \mathrm{~L}_{\psi_{N S}}^{-1}\left[\mathscr{P}_{1} \xi^{1} \mathscr{P}_{0} \partial_{x} \mathrm{~W}_{0}\right]\right) d x\left(\equiv I_{5}\right) \\
& -\int_{\mathbb{R}} \cosh \left(\varepsilon x / D_{1}\right)\left(\mathscr{P}_{1} \xi^{1} \mathrm{~W}_{0},\left(\mathrm{~L}_{\psi_{N S}}^{-1}\right)_{x}\left[\mathscr{P}_{1} \xi^{1} \mathscr{P}_{0} \partial_{x} \mathrm{~W}_{0}\right]\right) d x\left(\equiv I_{6}\right) \\
& \quad+\int_{\mathbb{R}} \cosh \left(\varepsilon x / D_{1}\right) O(1) \sum_{j=1}^{3} \varepsilon^{2}\left|W_{0}^{j}\right|^{2}\left|\lambda_{j}\right| e^{-\varepsilon|x|} d x\left(\equiv I_{7}\right) \\
& \quad-\int_{\mathbb{R}} \cosh \left(\varepsilon x / D_{1}\right)\left(\mathrm{W}_{0}, \mathscr{P}_{0} \xi^{1} \mathrm{~L}_{\psi_{N S}}^{-1}\left(\mathrm{~L}_{\varphi}-\mathrm{L}_{\psi_{N S}}\right) \mathrm{C}\right) d x\left(\equiv I_{8}\right) \\
& -\int_{\mathbb{R}} \cosh \left(\varepsilon x / D_{1}\right)\left[\left(\mathrm{W}_{0}, \mathscr{P}_{0} \xi^{1} \mathrm{~L}_{\psi_{N S}}^{-1} \overline{\mathrm{S}}\right)+\left(\mathrm{W}_{0}, \mathscr{P}_{0} \check{\boldsymbol{S}}\right)\right] d x\left(\equiv I_{9}\right) \\
& +\int_{\mathbb{R}} \cosh \left(\varepsilon x / D_{1}\right)\left(\mathrm{W}_{0}, \mathscr{P}_{0} \xi^{1} \mathrm{~L}_{\psi_{N S}}^{-1}\left[\partial_{t} \mathscr{P}_{1} \mathrm{C}+\partial_{x} \xi^{1} \mathscr{P}_{1} \mathrm{C}\right]\right) d x\left(\equiv I_{10}\right) .
\end{aligned}
$$


From the fact (2.5) that $\mathrm{L}_{\psi_{N S}}$ is a negative-definite operator on the space of microscopic components, it follows that

$$
I_{5} \geq \nu_{1} \int_{\mathbb{R}} \cosh \left(\varepsilon x / D_{1}\right)\left\|\mathscr{P}_{1} \xi \mathscr{P}_{0} \partial_{x} \mathrm{~W}_{0}\right\|_{L_{\xi}^{2}}^{2} d x \text { for some constant } \nu_{1}>0 .
$$

Since the local Maxwellian states are based on both the velocity profile $\bar{u}(x)$ and the temperature profile $\overline{\boldsymbol{T}}(x)$ of a compressible Navier-Stokes shock profile, there exists $A_{1}>0$ such that the characteristic fields

$$
\left\{\lambda_{1}, \lambda_{2}, \lambda_{3}\right\}=\{\bar{u}(x)-\sqrt{5 \overline{\boldsymbol{T}}(x) / 3}, \bar{u}(x), \bar{u}(x)+\sqrt{5 \overline{\boldsymbol{T}}(x) / 3}\}
$$

satisfy

$$
\begin{aligned}
& \left\{\begin{aligned}
\left|\partial_{x} \lambda_{i}(x)\right| & \leq A_{1} \varepsilon^{2} e^{-\varepsilon|x| / D_{0}} \text { for } i=1,2 \\
\left\|\partial_{x} \psi_{N S}\right\|_{L_{\xi}^{2}} & \leq A_{1} \varepsilon^{2} e^{-\varepsilon|x| / D_{0}}
\end{aligned}\right. \\
& \partial_{x} \lambda_{3}(x) \equiv \partial_{x}(\bar{u}(x)+\sqrt{5 \overline{\boldsymbol{T}}(x) / 3}) \leq-\varepsilon^{2} e^{-\varepsilon|x| / D_{0}} / A_{1}<0, \\
& \left\{\begin{array}{l}
{\left[-\partial_{x} \lambda_{3}-\frac{\varepsilon \lambda_{3} \tanh \left(\varepsilon x / D_{1}\right)}{2 D_{1}}\right] \geq \frac{1}{A_{1}} \frac{\varepsilon^{2}}{D_{1}^{2}}>0 \text { for } D_{1} \geq 1,} \\
-\lambda_{3}(x) \tanh \left(\varepsilon x / D_{1}\right)>0 .
\end{array}\right.
\end{aligned}
$$

From the analytic properties of the characteristic fields $\left\{\lambda_{1}, \lambda_{2}, \lambda_{3}\right\}$ in (5.63) and (5.64), one has

$$
I_{1}+I_{2} \geq \int_{\mathbb{R}} \cosh \left(\varepsilon x / D_{1}\right)\left(\frac{\varepsilon}{4} \sum_{j=1}^{2}\left|W_{0}^{j}\right|^{2}-O(1) \varepsilon^{3}\left|W_{0}^{3}\right|^{2}\right) d x .
$$

From (5.65), one has that

$$
I_{3} \geq \frac{\varepsilon^{2}}{4 A_{1} D_{1}^{2}} \int_{\mathbb{R}} \cosh \left(\varepsilon x / D_{1}\right)\left|W_{0}^{3}(x, t)\right|^{2} d x>0,
$$

and from this, (5.62), and (5.67)

$$
\begin{aligned}
I_{4} \leq \int_{\mathbb{R}} \cosh \left(\varepsilon x / D_{1}\right) & \left(-\frac{\varepsilon^{3 / 2} \lambda_{3}(x) \tanh \left(\varepsilon x / D_{1}\right)}{D_{1}}\left\|\mathrm{~W}_{0}\right\|_{L_{\xi}^{2}}^{2}\right. \\
& \left.+\varepsilon^{1 / 2} \frac{\left\|\mathscr{P}_{1} \xi^{1} \mathscr{P}_{0} \partial_{x} \mathrm{~W}_{0}\right\|_{L_{\xi}^{2}}^{2}}{D_{1}}\right) d x \leq O(1) \varepsilon^{1 / 2}\left(I_{3}+I_{5}\right) .
\end{aligned}
$$

From (5.63), (5.66), (5.67), and (5.62)

$$
\begin{aligned}
& \int_{\mathbb{R}} \cosh \left(\varepsilon x / D_{1}\right)\left|\left(\mathscr{P}_{1} \xi^{1} \mathrm{~W}_{0},\left(\mathrm{~L}_{\psi_{N S}}^{-1}\right)_{x} \mathscr{P}_{1} \xi^{1} \mathscr{P}_{0} \partial_{x} \mathrm{~W}_{0}\right)\right| d x \\
& \quad \leq \int_{\mathbb{R}} \cosh \left(\varepsilon x / D_{1}\right)\left(\varepsilon^{3}\left\|\mathrm{~W}_{0}\right\|_{L_{\xi}^{2}}^{2}+\varepsilon\left\|\mathscr{P}_{1} \xi^{1} \partial_{x} \mathscr{P}_{0} \mathrm{~W}_{0}\right\|_{L_{\xi}^{2}}^{2}\right) d x \\
& \quad \leq O(1) \varepsilon\left(I_{1}+I_{2}+I_{3}+I_{5}\right) .
\end{aligned}
$$

From (5.66) and (5.67),

$$
I_{7} \leq O(1) \varepsilon\left(I_{1}+I_{2}+I_{3}\right)
$$


From [1] (i.e. $\left\|\varphi(x)-\psi_{N S}(x)\right\|_{L_{\xi}^{2}} \leq O(1) \varepsilon^{2} e^{-\varepsilon|x| / D_{0}}$ ) and (5.66), (5.67) one has that

$$
\begin{aligned}
I_{8} \leq O(1) \int_{\mathbb{R}} \cosh \left(\varepsilon|x| / D_{1}\right)\left(\varepsilon^{3}\left\|\mathrm{~W}_{0}\right\|_{L_{\xi}^{2}}^{2}+O(1) \varepsilon\left\|\mathscr{P}_{1} \mathrm{C}\right\|_{L_{\xi}^{2}}^{2}\right) d x \\
\leq O(1) \varepsilon\left(I_{1}+I_{2}+I_{3}+\int_{\mathbb{R}} \cosh \left(\varepsilon x / D_{1}\right)\left\|\mathscr{P}_{1} \mathrm{C}\right\|_{L_{\xi}^{2}}^{2} d x\right) .
\end{aligned}
$$

From (5.44), (5.45), (5.46), and (5.51),

$$
\left|\left(\mathrm{W}_{0}, \xi^{1} \mathrm{~L}_{\psi_{N S}}^{-1} \overline{\mathrm{S}}\right)\right|,\left|\left(\mathrm{W}_{0}, \check{\boldsymbol{S}}\right)\right| \leq\left(\frac{\varepsilon^{2}}{D_{1}^{3}}\left\|\mathrm{~W}_{0}\right\|_{L_{\xi}^{2}}^{2}+O(1) \varepsilon^{-42 / 20} \frac{e^{-\frac{2 \varepsilon|x|}{D_{1}}-\frac{2 \varepsilon^{2} t}{E_{0}}}}{(1+t)^{\alpha}}\right)
$$

This, (5.66), and (5.67) result in

$$
I_{9} \leq O(1) \frac{I_{1}+I_{2}+I_{3}}{D_{1}}+O(1) \varepsilon^{-62 / 20} \frac{e^{-\frac{2 \varepsilon^{2} t}{E_{0}}}}{(1+t)^{\alpha}} .
$$

Thus, (5.66), (5.67), (5.62), (5.68), (5.70), (5.71), and (5.73) result in

$$
\begin{aligned}
& \frac{1}{2} \frac{d}{d t} \int_{\mathbb{R}} \cosh \left(\frac{\varepsilon x}{D_{1}}\right)\left\|\mathrm{W}_{0}\right\|_{L_{\xi}^{2}}^{2} d x \\
& +\int_{\mathbb{R}} \frac{\varepsilon^{2}}{8 A_{1} D_{1}^{2}} \cosh \left(\frac{\varepsilon x}{D_{1}}\right)\left\|\mathrm{W}_{0}\right\|_{L_{\xi}^{2}}^{2} d x+\frac{\nu_{1}}{2} \int_{\mathbb{R}} \cosh \left(\frac{\varepsilon x}{D_{1}}\right)\left\|\mathscr{P}_{1} \xi^{1} \partial_{x} \mathrm{~W}_{0}\right\|_{L_{\xi}^{2}}^{2} d x \\
& +\int_{\mathbb{R}} \cosh \left(\frac{\varepsilon x}{D_{1}}\right)\left[\left(\mathrm{W}_{0}, \mathscr{P}_{0} \xi^{1} \mathrm{~L}_{\psi_{N S}}^{-1}\left(\partial_{t}\left(\mathscr{P}_{1} \mathrm{C}\right)+\partial_{x}\left(\xi^{1} \mathscr{P}_{1} \mathrm{C}\right)\right)\right)\right] d x \\
& \leq O(1) \frac{\varepsilon^{-62 / 20}}{(1+t)^{\alpha}} e^{-2 \varepsilon^{2} t / E_{0}} .
\end{aligned}
$$

For the term $\int_{\mathbb{R}} \cosh \left(\frac{\varepsilon x}{D_{1}}\right)\left[\left(\mathrm{W}_{0}, \mathscr{P}_{0} \xi^{1} \mathrm{~L}_{\psi_{N S}}^{-1}\left(\partial_{t}\left(\mathscr{P}_{1} \mathrm{C}\right)+\partial_{x}\left(\xi^{1} \mathscr{P}_{1} \mathrm{C}\right)\right)\right)\right] d x$ in (‥74) one splits it into two terms. First, by integration by part,

$$
\begin{gathered}
\int_{\mathbb{R}} \cosh \left(\varepsilon x / D_{1}\right)\left(\mathrm{W}_{0}, \mathscr{P}_{0} \xi^{1} \mathrm{~L}_{\psi_{N S}}^{-1} \partial_{t} \mathscr{P}_{1} \mathrm{C}\right) d x \\
=\frac{d}{d t} \int_{\mathbb{R}} \cosh \left(\varepsilon x / D_{1}\right)\left(\mathrm{W}_{0}, \mathscr{P}_{0} \xi^{1} \mathrm{~L}_{\psi_{N S}}^{-1} \mathscr{P}_{1} \mathrm{C}\right) d x \\
\quad-\int_{\mathbb{R}} \cosh \left(\varepsilon x / D_{1}\right)\left(\partial_{t} \mathrm{~W}_{0}, \mathscr{P}_{0} \xi^{1} \mathrm{~L}_{\psi_{N S}}^{-1} \mathscr{P}_{1} \mathrm{C}\right) d x \\
=\frac{d}{d t} \int_{\mathbb{R}} \cosh \left(\varepsilon x / D_{1}\right)\left(\mathrm{W}_{0}, \mathscr{P}_{0} \xi^{1} \mathrm{~L}_{\psi_{N S}}^{-1} \mathscr{P}_{1} \mathrm{C}\right) d x+\int_{\mathbb{R}} \cosh \left(\varepsilon x / D_{1}\right) \\
\cdot\left(\mathscr{P}_{0} \xi^{1}\left(\mathscr{P}_{0} \partial_{x} \mathrm{~W}_{0}+\mathscr{P}_{1} \mathrm{C}\right)+\varepsilon \sum_{j=1}^{2}\left(\mathrm{I}_{j}, \mathrm{~W}_{0}\right) \mathscr{P}_{0} \mathrm{r}_{j}-\mathscr{P}_{0} \check{S}, \mathscr{P}_{0} \xi^{1} \mathrm{~L}_{\psi_{N S}}^{-1} \mathscr{P}_{1} \mathrm{C}\right) d x
\end{gathered}
$$


and it yields that

$$
\begin{aligned}
& \frac{d}{d t} \int_{\mathbb{R}} \cosh \left(\frac{\varepsilon x}{D_{1}}\right)\left(\mathrm{W}_{0}, \mathscr{P}_{0} \xi^{1} \mathrm{~L}_{\psi_{N S}}^{-1} \mathscr{P}_{1} \mathrm{C}\right) d x \\
&-\int_{\mathbb{R}} \cosh \left(\frac{\varepsilon x}{D_{1}}\right)\left(\mathrm{W}_{0}, \mathscr{P}_{0} \xi^{1} \mathrm{~L}_{\psi_{N S}}^{-1} \partial_{t} \mathscr{P}_{1} \mathrm{C}\right) d x \leq O(1) \varepsilon\left(I_{1}+I_{2}+I_{3}\right) \\
&+O(1) \int_{\mathbb{R}} \cosh \left(\frac{\varepsilon x}{D_{1}}\right)\left(\frac{\left\|\mathscr{P}_{1} \xi^{1} \mathscr{P}_{0} \mathrm{~W}_{0 x}\right\|_{L_{\xi}^{2}}^{2}}{D_{1}}+D_{1}\left\|\mathscr{P}_{1} \mathrm{C}\right\|_{L_{\xi}^{2}}^{2}\right) d x \\
&+O(1) \frac{\varepsilon^{-22 / 20} e^{-2 \varepsilon^{2} t / E_{0}}}{D_{1}(1+t)^{\alpha}} .
\end{aligned}
$$

Second,

$$
\begin{gathered}
\int_{\mathbb{R}} \cosh \left(\varepsilon x / D_{1}\right)\left(\mathrm{W}_{0}, \mathscr{P}_{0} \xi^{1} \mathrm{~L}_{\psi_{N S}}^{-1} \partial_{x} \xi^{1} \mathscr{P}_{1} \mathrm{C}\right) d x \\
=-\int_{\mathbb{R}} \cosh \left(\varepsilon x / D_{1}\right)\left(\mathscr{P}_{1} \xi^{1} \partial_{x} \mathrm{~W}_{0}, \mathrm{~L}_{\psi_{N S}}^{-1} \xi^{1} \mathscr{P}_{1} \mathrm{C}\right) d x \\
-\int_{\mathbb{R}} \cosh \left(\varepsilon x / D_{1}\right)\left(\mathrm{W}_{0}, \xi^{1}\left(\left(\mathrm{~L}_{\psi_{N S}}^{-1}\right)_{x}+\frac{\varepsilon \tanh \left(\varepsilon x / D_{1}\right)}{D_{1}} \mathrm{~L}_{\psi_{N S}}^{-1}\right) \xi^{1} \mathscr{P}_{1} \mathrm{C}\right) d x \\
\leq O(1) \sqrt{\varepsilon}\left(I_{1}+I_{2}+I_{3}\right) \\
+O(1) \int_{\mathbb{R}} \cosh \left(\varepsilon x / D_{1}\right)\left(\frac{\left\|\mathscr{P}_{1} \xi^{1} \mathscr{P}_{0} \mathrm{~W}_{0}\right\|_{L_{\xi}^{2}}^{2}}{D_{1}}+D_{1}\left\|\mathscr{P}_{1} \mathrm{C}\right\|_{L_{\xi}^{2}}^{2}\right) d x
\end{gathered}
$$

Then one combines (5.74), (5.76), and (5.77) together to conclude this proposition.

Similarly to Proposition 5.6, from (5.43) one has the energy estimate

$$
\begin{array}{r}
\frac{d}{d t} \int_{\mathbb{R}} \cosh \left(\varepsilon x / D_{1}\right) \frac{(\mathrm{C}, \mathrm{C})}{2} d x+\int_{\mathbb{R}} \cosh \left(\varepsilon x / D_{1}\right)\left[-\frac{\varepsilon \tanh \left(\varepsilon x / D_{1}\right)}{D_{1}} \frac{\left(\mathrm{C}, \xi^{1} \mathrm{C}\right)}{2}\right. \\
\left.-\left(\mathrm{C}, \mathrm{L}_{\psi_{N S}} \mathrm{C}\right)+\varepsilon \sum_{j=1}^{2}\left(\mathrm{r}_{j}, \mathrm{C}\right)\left(\mathrm{C}, \mathrm{r}_{j}\right)+\left(\mathrm{C},\left(\mathrm{L}_{\psi_{N S}}-\mathrm{L}_{\varphi}\right) \mathrm{C}\right)\right] d x \\
-\int_{\mathbb{R}} \cosh \left(\varepsilon x / D_{1}\right)(\mathrm{C}, \overline{\mathrm{S}}) d x=0
\end{array}
$$

to yield the proposition:

\section{Proposition 5.7.}

$$
\begin{aligned}
& \frac{d}{d t} \int_{\mathbb{R}} \cosh \left(\varepsilon x / D_{1}\right) \frac{(\mathrm{C}, \mathrm{C})}{2} d x \\
+ & \int_{\mathbb{R}} \cosh \left(\varepsilon x / D_{1}\right)\left(\frac{\varepsilon\left[\left(\mathrm{I}_{1}, \mathrm{C}\right)^{2}+\left(\mathrm{I}_{2}, \mathrm{C}\right)^{2}\right]+\nu_{1}\left\|\mathscr{P}_{1} \mathrm{C}\right\|_{L_{\xi}^{2}}^{2}}{2}-\frac{\varepsilon^{2}\left|\left(\mathrm{I}_{3}, \mathrm{C}\right)\right|^{2}}{A_{1} D_{1}^{2}}\right) d x \\
\leq & O(1) \int_{\mathbb{R}} \cosh \left(\varepsilon x / D_{1}\right)\left(\varepsilon^{-2}\left\|\mathrm{P}_{0}^{-} \overline{\mathrm{S}}\right\|_{L_{\xi}^{2}}^{2}+\left\|\mathrm{P}_{1}^{-} \overline{\mathrm{S}}\right\|_{L_{\xi}^{2}}^{2}\right) d x \leq O(1) \varepsilon^{-22 / 20} \frac{e^{-2 \varepsilon^{2} t / E_{0}}}{(1+t)^{\alpha}} .
\end{aligned}
$$


By combining (5.60) and (5.79) together, we have the proposition:

\section{Proposition 5.8.}

$$
\begin{gathered}
\left.\int_{\mathbb{R}} \cosh \left(\varepsilon x / D_{1}\right)\left[\varepsilon^{2}|\log \varepsilon|\left(\frac{\left\|\mathrm{W}_{0}\right\|_{L_{\xi}^{2}}^{2}}{2}+\left(\xi^{1} \mathrm{~W}_{0}, \mathrm{~L}_{\psi_{N S}}^{-1} \mathscr{P}_{1} \mathrm{C}\right)\right)+(\mathrm{C}, \mathrm{C})\right] e^{\frac{\varepsilon^{2} t}{E_{1}}} d x\right|_{t=0} ^{t=\tau} \\
\quad+\int_{0}^{\tau} \int_{\mathbb{R}} \cosh \left(\varepsilon x / D_{1}\right) \\
\cdot\left[\varepsilon^{2}|\log \varepsilon| \frac{\nu_{1}\left\|\mathscr{P}_{1} \xi^{1} \mathscr{P}_{0} \partial_{x} \mathrm{~W}_{0}\right\|_{L_{\xi}^{2}}^{2}}{2}+\frac{\nu_{1}\left(\mathscr{P}_{1} \mathrm{C}, \mathscr{P}_{1} \mathrm{C}\right)}{2}+\frac{\varepsilon \sum_{j=1}^{2}\left(\mathrm{I}_{j}, \mathrm{C}\right)^{2}}{4}\right] e^{\frac{\varepsilon^{2} t}{E_{1}}} d x d t \\
\leq O(1) \varepsilon^{-62 / 20} \frac{e^{\left(\frac{1}{E_{1}}-\frac{2}{E_{0}}\right) \varepsilon^{2} \tau}}{(1+\tau)^{\alpha}}+\frac{\varepsilon^{2}}{A_{1} D_{1}^{2}} \int_{0}^{\tau} \int_{\mathbb{R}} \cosh \left(\varepsilon x / D_{1}\right)\left(\mathrm{I}_{3}, \mathrm{C}\right)^{2} e^{\frac{\varepsilon^{2} t}{E_{1}}} d x d t,
\end{gathered}
$$

where $E_{1} \equiv 8 A_{1} D_{1}^{2}$ and by letting $E_{0}$ be large enough to satisfy

$$
E_{1} \equiv 8 A_{1} D_{1}^{2}<\frac{1}{2} E_{0}
$$

Lemma 5.9. For the functions $\mathscr{I}_{\alpha}$ and $\tilde{\mathscr{I}}_{\alpha}$ defined in (5.2) and (5.3) with $\alpha \in$ $[1,2]$, the functions $\mathrm{C}(x, t) \equiv \mathbb{C}_{3}\left[\mathbb{R}_{3}\left[\mathscr{I}_{\alpha}\right]\right]$ and $\tilde{\mathbb{C}} \equiv \mathbb{C}_{3}\left[\mathbb{R}_{3}\left[\tilde{\mathscr{I}}_{\alpha}\right]\right]$ satisfy

$$
\begin{array}{r}
\left.\int_{\mathbb{R}} \cosh \left(\varepsilon x / D_{1}\right)(\mathrm{C}, \mathrm{C}) d x\right|_{t=\tau} \leq O(1) \varepsilon^{-62 / 20} \frac{e^{-2 \varepsilon^{2} \tau / E_{0}}}{(1+\tau)^{\alpha}} \\
\left.\int_{\mathbb{R}} \cosh \left(\varepsilon x / D_{1}\right)(\tilde{\mathrm{C}}, \tilde{\mathrm{C}}) d x\right|_{t=\tau} \leq O(1) \varepsilon^{-62 / 20} \frac{\left(e^{-2 \varepsilon \tau / E_{0}}+\varepsilon^{\frac{1}{2}} e^{-2 \varepsilon^{2} \tau / E_{0}}\right)}{(1+\tau)^{\alpha}}
\end{array}
$$

Proof. Since the proofs for (5.81) and (5.82) are almost identical, we just prove (5.81).

In order to continue from Proposition 5.8 to complete the low order energy estimates, one still needs to estimate $\int_{0}^{\tau} \int_{\mathbb{R}} \cosh \left(\varepsilon x / D_{1}\right)\left(\mathrm{I}_{3}, \mathrm{C}\right)^{2} e^{\frac{\varepsilon^{2} t}{E_{1}}} d x d t$ in the RHS of (5.80). We introduce the variables $\left(a_{1}, a_{2}, a_{2}\right)$ to decompose $W_{0}$ and use conservation laws to estimate the double integral in the RHS of (5.80):

$$
\begin{aligned}
& \left\{\begin{array}{l}
\mathrm{a}_{1} \equiv\left(\sqrt{\mathrm{M}_{-}}, \mathrm{W}_{0}\right), \\
\bar{u}(x) \mathrm{a}_{1}+\mathrm{a}_{2} \sqrt{\overline{\boldsymbol{T}}(x)} \equiv\left(\xi^{1} \sqrt{\mathrm{M}_{-}}, \mathrm{W}_{0}\right), \\
\left(3 \sqrt{6} \overline{\boldsymbol{T}}(x)+\bar{u}(x)^{2}\right) \mathrm{a}_{1}+2 \bar{u}(x) \sqrt{\overline{\boldsymbol{T}}(x)} \mathrm{a}_{2}+\sqrt{6} \overline{\boldsymbol{T}}(x) \mathrm{a}_{3} \\
\equiv\left(|\xi|^{2} \sqrt{\mathrm{M}_{-}}, \mathrm{W}_{0}\right),
\end{array}\right. \\
& \mathrm{W}_{0}(x, t)=\left(\mathrm{a}_{1}(x, t)+\mathrm{a}_{2}(x, t) \frac{\left(\xi^{1}-\bar{u}(x)\right)}{\sqrt{\overline{\boldsymbol{T}}(x)}}\right. \\
& \left.+\mathrm{a}_{3}(x, t) \frac{1}{\sqrt{6}}\left(\frac{\left|\xi^{1}-\bar{u}(x)\right|^{2}+\left|\xi^{2}\right|^{2}+\left|\xi^{3}\right|^{2}}{\overline{\boldsymbol{T}}(x)}-3\right)\right) \frac{\mathrm{M}_{[1, \bar{u}(x), \overline{\boldsymbol{T}}(x)]}}{\sqrt{\mathrm{M}_{-}}} .
\end{aligned}
$$


With those variables $\left(a_{1}, a_{2}, a_{3}\right),(5.54)$ can be rewritten as

$$
\left\{\begin{array}{c}
\partial_{t} \mathrm{a}_{1}+\partial_{x}\left(\bar{u} \mathrm{a}_{1}+\sqrt{\overline{\boldsymbol{T}}} \mathrm{a}_{2}\right)=O(1) \varepsilon\left\|\mathrm{W}_{0}\right\|_{L_{\xi}^{2}}+O(1) \varepsilon^{-1 / 20} \frac{-\frac{3 \varepsilon|x|}{D_{1}}-\frac{\varepsilon^{2} t}{E_{0}}}{(1+t)^{\alpha / 2}} \\
\partial_{t}\left(\bar{u} \mathrm{a}_{1}+\sqrt{\overline{\boldsymbol{T}}} \mathrm{a}_{2}\right)+\partial_{x}\left(\bar{u}^{2} \mathrm{a}_{1}+2 \sqrt{\overline{\boldsymbol{T}}} \overline{\mathrm{a}_{2}}+\overline{\boldsymbol{T}} \sqrt{\frac{3}{2}} \mathrm{a}_{3}\right)+O(1)\left\|\mathscr{P}_{1} \mathrm{C}\right\|_{L_{\xi}^{2}} \\
=O(1) \varepsilon\left\|\mathrm{W}_{0}\right\|_{L_{\xi}^{2}}+O(1) \varepsilon^{-1 / 20} \frac{-\frac{3 \varepsilon|x|}{D_{1}}-\frac{\varepsilon^{2} t}{E_{0}}}{(1+t)^{\alpha / 2}} \\
\partial_{t}\left(\left(3 \sqrt{6} \overline{\boldsymbol{T}}+\bar{u}^{2}\right) \mathrm{a}_{1}+2 \bar{u} \sqrt{\overline{\boldsymbol{T}}} \mathrm{a}_{2}+\sqrt{6} \overline{\boldsymbol{T}} \mathrm{a}_{3}\right)+\ldots
\end{array}\right.
$$

From the first two equations in (5.85), we have

$$
\begin{gathered}
0=\int_{0}^{\tau} \int_{\mathbb{R}} e^{\varepsilon^{2} t / E_{1}} \cosh \left(\varepsilon x / D_{1}\right)\left(\mathrm{a}_{1}\right)_{x}\left[\partial_{t}\left(\left(\mathrm{a}_{1} \bar{u}+\mathrm{a}_{2}\right) \sqrt{\overline{\boldsymbol{T}}}\right)\right. \\
+\partial_{x}\left(\bar{u}^{2} \mathrm{a}_{1}+2 \bar{u} \sqrt{\boldsymbol{T}} \mathrm{a}_{2}+\mathrm{a}_{3} \overline{\boldsymbol{T}} \sqrt{2 / 3}\right)+O(1)\left\|\mathscr{P}_{1} \mathrm{C}\right\|_{L_{\xi}^{2}}-O(1) \varepsilon\left\|\mathrm{W}_{0}\right\|_{L_{\xi}^{2}} \\
\left.+\varepsilon^{-1 / 20} \frac{-\frac{3 \varepsilon|x|}{D_{1}}-\frac{\varepsilon^{2} t}{E_{0}}}{(1+t)^{\alpha / 2}}\right] d x d t \\
\geq\left.\int_{\mathbb{R}} e^{\varepsilon^{2} t / E_{1}} \cosh \left(\varepsilon x / D_{1}\right)\left(\mathrm{a}_{1}\right)_{x}\left(\mathrm{a}_{1} \bar{u}+\mathrm{a}_{2}\right) \sqrt{\overline{\boldsymbol{T}}(x)} d x\right|_{0} ^{\tau} \\
+\int_{0}^{\tau} \int_{\mathbb{R}} e^{\varepsilon^{2} t / E_{1}} \cosh \left(\varepsilon x / D_{1}\right)\left(\bar{u}^{2} \frac{\left|\partial_{x} \mathrm{a}_{1}\right|^{2}}{2}\right. \\
\left.-\tilde{\mathscr{C}}\left[\left|\partial_{x} \mathrm{a}_{2}\right|^{2}+\left|\partial_{x} \mathrm{a}_{3}\right|^{2}+\left\|\mathscr{P}_{1} \mathrm{C}\right\|_{L_{\xi}^{2}}^{2}+\varepsilon^{2}\left\|\mathrm{~W}_{0}\right\|_{L_{\xi}^{2}}^{2}+\varepsilon^{-2 / 20} \frac{e^{-\frac{3 \varepsilon|x|}{D_{1}}-\frac{2 \varepsilon^{2} t}{E_{0}}}}{(1+t)^{\alpha}}\right]\right) d x d t,
\end{gathered}
$$

where the constant $\tilde{\mathscr{C}}>0$ depends on the ratio $\bar{u}^{2} / \overline{\boldsymbol{T}}$.

Due to (5.83), $\left|\mathrm{a}_{2}\right|^{2}+\left|\mathrm{a}_{3}\right|^{2}$ and $\left\|\mathscr{P}_{1} \xi^{1} \mathrm{~W}_{0}\right\|^{2}$ are equivalent; from (5.86) one has

$$
\begin{gathered}
\text { (5.87) }\left.\int_{\mathbb{R}} e^{\varepsilon^{2} t / E_{1}} \cosh \left(\varepsilon x / D_{1}\right)\left(\mathrm{a}_{1}\right)_{x}\left(\mathrm{a}_{1} \bar{u}+\mathrm{a}_{2}\right) \sqrt{\overline{\boldsymbol{T}}(x)} d x\right|_{t=\tau} \\
+\int_{0}^{\tau} \int_{\mathbb{R}} e^{\varepsilon^{2} t / E_{1}} \cosh \left(\varepsilon x / D_{1}\right) \bar{u}^{2} \frac{\left|\partial_{x} \mathrm{a}_{1}\right|^{2}}{2} d x d t \\
\leq\left.\int_{\mathbb{R}} e^{\varepsilon^{2} t / E_{1}} \cosh \left(\varepsilon x / D_{1}\right)\left|\left(\mathrm{a}_{1}\right)_{x}\left(\mathrm{a}_{1} \bar{u}+\mathrm{a}_{2}\right)\right| \sqrt{\overline{\boldsymbol{T}}(x)} d x\right|_{t=0} \\
+O(1) \int_{0}^{\tau} \int_{\mathbb{R}} e^{\varepsilon^{2} t / E_{1}} \cosh \left(\varepsilon x / D_{1}\right)\left(\left\|\mathscr{P}_{1} \xi^{1} \partial_{x} \mathrm{~W}_{0}\right\|_{L_{\xi}^{2}}^{2}+\left\|\mathscr{P}_{1} \mathrm{C}\right\|_{L_{\xi}^{2}}^{2}+\varepsilon^{2}\left\|\mathrm{~W}_{0}\right\|_{L_{\xi}^{2}}^{2}\right) d x d t \\
+O(1) e^{-62 / 20} \frac{e^{\left(\frac{1}{E_{1}}-\frac{2}{E_{0}}\right) \varepsilon^{2} \tau}}{(1+\tau)^{\alpha}} .
\end{gathered}
$$

Furthermore, due to the fact that $\mathscr{P}_{0} \mathrm{C}=\mathscr{P}_{0} \partial_{x} \mathrm{~W}_{0}$,

$$
\varepsilon^{2}\left(\mathrm{I}_{3}, \mathrm{C}\right)^{2} \leq O(1) \varepsilon^{2}\left\|\mathscr{P}_{0} \mathrm{C}\right\|_{L_{\xi}^{2}}^{2} \leq O(1) \varepsilon^{2}\left(\left|\partial_{x} \mathrm{a}_{1}\right|^{2}+\left\|\mathscr{P}_{1} \xi^{1} \partial_{x} \mathrm{~W}_{0}\right\|_{L_{\xi}^{2}}^{2}\right)
$$


Then combine this and $\varepsilon^{2}|\log \varepsilon|^{1 / 2} \cdot(\underline{5.87)}+[5.80$ together to yield that

$$
\left.\int_{\mathbb{R}} \cosh \left(\varepsilon x / D_{1}\right)(\mathrm{C}, \mathrm{C}) d x\right|_{t=\tau} \leq O(1) \varepsilon^{-62 / 20} \frac{e^{-2 \varepsilon^{2} \tau / E_{0}}}{(1+\tau)^{\alpha}} .
$$

The lemma follows.

5.4. Energy-pointwise estimates. With the low order energy estimates yielded by Lemma [5.9], one continues to obtain pointwise estimates together with the Green's function of $\left(\partial_{t}+\xi^{1} \partial_{x}-\mathrm{L}_{-}\right) \mathrm{h}+\varepsilon \sum_{j=1}^{2}\left(\mathrm{I}_{j}, \mathrm{~h}\right) \mathrm{r}_{j}=0$.

Theorem 5.10. The functions $C(x, t) \equiv \mathbb{C}_{3}\left[\mathbb{R}_{3}\left[\mathscr{I}_{\alpha}\right]\right]$ and $\tilde{\mathrm{C}}(x, t) \equiv \mathbb{C}_{3}\left[\mathbb{R}_{3}\left[\tilde{\mathscr{I}}_{\alpha}\right]\right]$ satisfy

$$
\begin{aligned}
& \varepsilon \sum_{k=1}^{2}\left|\left(\mathrm{I}_{k}, \mathrm{C}\right)\right| \leq O(1) \varepsilon^{1 / 5} \frac{e^{-\frac{\varepsilon^{2} t}{E_{0}}}}{(1+t)^{\alpha / 2}} e^{-3 \varepsilon|x| / D_{1}}, \\
& \varepsilon \sum_{k=1}^{2}\left|\left(\mathrm{I}_{k}, \tilde{\mathrm{C}}\right)\right| \leq O(1) \varepsilon^{1 / 5} \frac{\left(e^{-\frac{\varepsilon t}{E_{0}}}+\varepsilon^{\frac{1}{2}} e^{-\frac{\varepsilon^{2} t}{E_{0}}}\right)}{(1+t)^{\alpha / 2}} e^{-3 \varepsilon|x| / D_{1}}, \\
& \|\mathrm{C}(x, t)\|_{L_{\xi, 3}^{\infty}} \leq O(1) \varepsilon^{-21 / 20} \frac{e^{-\frac{\varepsilon^{2} t}{E_{0}}}}{(1+t)^{\alpha / 2}} e^{-3 \varepsilon|x| / D_{1}}, \\
& \|\tilde{\mathrm{C}}(x, t)\|_{L_{\xi, 3}^{\infty}} \leq O(1) \varepsilon^{-21 / 20} \frac{\left(e^{-\frac{\varepsilon t}{E_{0}}}+\varepsilon^{\frac{1}{2}} e^{-\frac{\varepsilon^{2} t}{E_{0}}}\right)}{(1+t)^{\alpha / 2}} e^{-3 \varepsilon|x| / D_{1}} .
\end{aligned}
$$

Proof. We only show this lemma for the case $x<0$ for (5.90) and (5.92), since the proofs for other cases are similar.

Denote the Green's functions of the following equations referring to the end states $\mathrm{M}_{ \pm}$by $\mathbb{G}_{ \pm}^{D, \varepsilon}(x, t)$ :

$$
\partial_{t} \mathrm{~h}+\xi^{1} \partial_{x} \mathrm{~h}-\mathrm{L}_{ \pm} \mathrm{h}+\varepsilon \sum_{k=1}^{2}\left(\mathrm{I}_{k}, \mathrm{~h}\right) \mathrm{r}_{k}=0
$$

Let $\mathrm{C}^{ \pm}(x, t)$ be the solution of

$$
\left\{\begin{array}{l}
\partial_{t} \mathrm{C}^{ \pm}+\xi^{1} \partial_{x} \mathrm{C}^{ \pm}-\mathrm{L}_{ \pm} \mathrm{C}^{ \pm}+\varepsilon \sum_{k=1}^{2}\left(\mathrm{I}_{k}, \mathrm{C}^{ \pm}\right) \mathrm{r}_{k}=\overline{\mathrm{S}}(x, t) \\
\mathrm{C}^{ \pm}(x, 0) \equiv 0
\end{array}\right.
$$

where the function $\overline{\mathrm{S}}$ satisfies (5.44); and one can represent the solution by the Duhamel principle

$$
\mathrm{C}^{-}(x, t)=\int_{0}^{t} \int_{\mathbb{R}} \mathbb{G}_{-}^{D, \varepsilon}(x-y, t-s) \overline{\mathrm{S}}(y, s) d y d s,
$$

where $\bar{S}$ is given in (5.44). Then one can apply Corollary 3.1 and (5.44) to yield 
that for $x<0$,

$$
\begin{aligned}
& \left|\left(\mathrm{I}_{j}, \mathrm{C}^{-}(x, t)\right)\right| \\
& \leq O(1) \int_{0}^{t} \int_{\mathbb{R}}\left(\sum_{k=1}^{2} \frac{e^{-\frac{\left(x-y-\lambda_{k}^{-}(t-s)\right)^{2}}{D_{0}(t-s)}-\varepsilon(t-s)}}{(t-s+1)^{1 / 2}}+\frac{e^{-\frac{(x-y-\varepsilon(t-s))^{2}}{D_{0}(t-s)}}}{(t-s+1)}\right) \\
& \cdot \varepsilon^{39 / 20} \frac{e^{-\frac{\varepsilon^{2} s}{E_{0}}}}{(1+s)^{\alpha / 2}} e^{-3 \varepsilon|y| / D_{1}} d y d s \\
& +O(1) \int_{0}^{t} \int_{\mathbb{R}}\left(\sum_{j=1}^{2} \frac{e^{-\frac{\left(x-y-\lambda_{j}^{-}(t-s)\right)^{2}}{D_{0}(t-s)}-\varepsilon(t-s)}}{(t-s+1)}+\frac{e^{-\frac{(x-y-\varepsilon(t-s))^{2}}{D_{0}(t-s)}}}{(t-s+1)^{3 / 2}}\right) \\
& \cdot \frac{e^{-\frac{\varepsilon^{2} s}{E_{0}}}}{(1+s)^{\alpha / 2}} e^{-3 \varepsilon|y| / D_{1}} d y d s \leq O(1) \varepsilon^{-1 / 2} \frac{e^{-\frac{3 \varepsilon|x|}{D_{1}}-\frac{\varepsilon^{2} t}{E_{0}}}}{(1+t)^{\alpha / 2}} \text { for } j=1,2 \text {, }
\end{aligned}
$$

and

$$
\begin{aligned}
\left|\left(I_{3}, \mathrm{C}^{-}(x, t)\right)\right| \leq O(1) \int_{0}^{t} \int_{\mathbb{R}}\left(\sum_{j=1}^{2} \frac{e^{-\frac{\left(x-y-\lambda_{j}^{-}(t-s)\right)^{2}}{D_{0}(t-s)}-\varepsilon(t-s)}}{(t-s+1)^{3 / 2}}+\frac{e^{-\frac{(x-y-\varepsilon(t-s))^{2}}{D_{0}(t-s)}}}{(t-s+1)^{\frac{1}{2}}}\right) \\
\cdot \varepsilon^{19 / 20} \frac{e^{-\frac{\varepsilon^{2} s}{D_{0}}}}{(1+s)^{\alpha / 2}} e^{-3 \varepsilon|y| / D_{1}} d y d s \\
+O(1) \int_{0}^{t} \int_{\mathbb{R}}\left(\sum_{j=1}^{2} \frac{e^{-\frac{\left(x-y-\lambda_{j}^{-}(t-s)\right)^{2}}{D_{0}(t-s)}-\varepsilon(t-s)}}{(t-s+1)^{3 / 2}}+\frac{e^{-\frac{(x-y-\varepsilon(t-s))^{2}}{D_{0}(t-s)}}}{(t-s+1)}\right) \\
\cdot \frac{e^{-\frac{\varepsilon^{2} s}{E_{0}}}}{(1+s)^{\alpha / 2}} e^{-3 \varepsilon|y| / D_{1}} d y d s \leq O(1) \varepsilon^{-21 / 20} \frac{e^{-\frac{3 \varepsilon|x|}{D_{1}}-\frac{\varepsilon^{2} t}{E_{0}}}}{(1+t)^{\alpha / 2}} .
\end{aligned}
$$

Let $\mathrm{D}^{ \pm}(x, t)$ be the difference between $\mathrm{C}^{ \pm}$and $\mathrm{C}$ :

$$
\mathrm{D}^{ \pm}(x, t) \equiv \mathrm{C}(x, t)-\mathrm{C}^{ \pm}(x, t) .
$$

From (5.43) and (5.94),

$$
\partial_{t} \mathrm{D}^{-}+\xi^{1} \partial_{x} \mathrm{D}^{-}-\mathrm{L}_{-} \mathrm{D}^{-}+\varepsilon \sum_{j=1}^{2}\left(\mathrm{I}_{j}, \mathrm{D}^{-}\right) \mathrm{r}_{j}=\left(\mathrm{L}_{\varphi}-\mathrm{L}_{-}\right) \mathrm{C} .
$$

From (5.81), Lemma A.9, and Lemma A.8, one has that for $l=1,2$ for $x<0$

$$
\begin{gathered}
\left|\left(\mathrm{I}_{l}, \mathrm{D}^{-}(x, t)\right)\right| \\
\leq O(1) \int_{0}^{t} \int_{\mathbb{R}}\left(\sum_{j=1}^{2} \frac{e^{-\frac{\left(x-y-\lambda_{j}^{-}(t-s)\right)^{2}}{D_{0}(t-s)}-\varepsilon(t-s)}}{(t-s+1)}+\frac{e^{-\frac{(x-y-\varepsilon(t-s))^{2}}{D_{0}(t-s)}}}{(t-s+1)^{3 / 2}}\right) \\
\cdot\left\|\left(\mathrm{L}_{\varphi}-\mathrm{L}_{-}\right) \mathrm{C}(y, s)\right\|_{L_{\xi}^{2}} d y d s
\end{gathered}
$$




$$
\begin{aligned}
& \leq O(1) \varepsilon \int_{0}^{t} \int_{-\infty}^{0}\left[\left(\sum_{j=1}^{2} \frac{e^{-\frac{\left(x-y-\lambda_{j}^{-}(t-s)\right)^{2}}{D_{0}(t-s)}-\varepsilon(t-s)}}{(t-s+1)}+\frac{e^{-\frac{(x-y-\varepsilon(t-s))^{2}}{D_{0}(t-s)}}}{(t-s+1)^{3 / 2}}\right) e^{-\frac{9 \varepsilon|y|}{2 D_{1}}}\right] \\
& \cdot\left[e^{\frac{\varepsilon|y|}{2 D_{1}}}\|\mathrm{C}(y, s)\|_{L_{\xi}^{2}}\right] d y d s \\
& +O(1) \varepsilon \int_{0}^{t} \int_{0}^{\infty}\left[\left(\sum_{j=1}^{2} \frac{e^{-\frac{\left(x-y-\lambda_{j}^{-}(t-s)\right)^{2}}{D_{0}(t-s)}-\varepsilon(t-s)}}{(t-s+1)}+\frac{e^{-\frac{(x-y-\varepsilon(t-s))^{2}}{D_{0}(t-s)}}}{(t-s+1)^{3 / 2}}\right) e^{-\frac{\varepsilon|y|}{2 D_{1}}}\right] \\
& \cdot\left[e^{\frac{\varepsilon|y|}{2 D_{1}}}\|\mathrm{C}(y, s)\|_{L_{\xi}^{2}}\right] d y d s \\
& \leq O(1) \varepsilon e^{3 \varepsilon x / D_{1}} \int_{0}^{t}\left(\frac{e^{-\varepsilon(t-s)}}{(t-s+1)^{3 / 2}}+\frac{e^{-\varepsilon^{2}(t-s) / D_{0}}}{(t-s+1)^{5 / 2}}\right)^{1 / 2} \varepsilon^{-31 / 20} \frac{e^{-\varepsilon^{2} s / E_{0}}}{(1+s)^{\alpha / 2}} d s \\
& \leq O(1) \varepsilon^{-11 / 20} e^{3 \varepsilon x / D_{1}} \int_{0}^{t}\left(\frac{e^{-\varepsilon(t-s)}}{(t-s+1)^{3 / 4}}+\frac{e^{-\varepsilon^{2}(t-s) / D_{0}}}{(t-s+1)^{5 / 4}}\right) \frac{e^{-\varepsilon^{2} s / E_{0}}}{(1+s)^{\alpha / 2}} d s \\
& \leq O(1) \varepsilon^{-\frac{4}{5}} e^{3 \varepsilon x / D_{1}} \frac{e^{-|\varepsilon|^{2} t / E_{0}}}{(1+t)^{\alpha / 2}}
\end{aligned}
$$

and one also has that for $x<0$

$$
\begin{array}{r}
\left|\left(\mathrm{I}_{3}, \mathrm{D}^{-}(x, t)\right)\right| \leq O(1) \int_{0}^{t} \int_{\mathbb{R}}\left(\sum_{j=1}^{2} \frac{e^{-\frac{\left(x-y-\lambda_{j}^{-}(t-s)\right)^{2}}{D_{0}(t-s)}-\varepsilon(t-s)}}{(t-s+1)^{3 / 2}}+\frac{e^{-\frac{(x-y-\varepsilon(t-s))^{2}}{D_{0}(t-s)}}}{(t-s+1)}\right) \\
\cdot\left\|\left(\mathrm{L}_{\varphi}-\mathrm{L}_{-}\right) \mathrm{C}(y, s)\right\|_{L_{\xi}^{2}} d y d s \\
\leq O(1) \varepsilon e^{3 \varepsilon x / D_{1}} \int_{0}^{t}\left(\frac{e^{-\varepsilon(t-s)}}{(t-s+1)^{5 / 4}}+\frac{e^{-\varepsilon^{2}(t-s) / D_{0}}}{(t-s+1)^{3 / 4}}\right) \varepsilon^{-31 / 20} \frac{e^{-\varepsilon^{2} s / E_{0}}}{(1+s)^{\alpha / 2}} d s \\
\leq O(1) \varepsilon^{-21 / 20}|\log \varepsilon| e^{\frac{3 \varepsilon x}{D_{1}}} \frac{e^{-\varepsilon^{2} t / E_{0}}}{(1+t)^{\alpha / 2}} .
\end{array}
$$

From (5.99) one has that for $l=1,2$

$$
\varepsilon\left|\left(\mathrm{I}_{j}, \mathrm{D}^{-}(x, t)\right)\right| \leq O(1) \varepsilon^{\frac{1}{5}} e^{\frac{3 \varepsilon x}{D_{1}}} \frac{e^{-|\varepsilon|^{2} t / E_{0}}}{(1+t)^{\alpha / 2}} \text { for } x<0 .
$$

From (5.101) and (5.96) one has that for $l=1,2$

$$
\varepsilon\left|\left(\mathrm{I}_{l}, \mathrm{C}(x, t)\right)\right| \leq \varepsilon^{1 / 5} e^{\frac{3 \varepsilon x}{D_{1}}} \frac{e^{-\varepsilon^{2} t / E_{0}}}{(1+t)^{\alpha / 2}} \text { for } x<0 .
$$

From this, (5.96), (5.97), and (5.100), one has that for $x<0$

$$
\left\|\mathrm{P}_{0}^{-} \mathrm{C}(x, t)\right\|_{L_{\xi}^{2}} \leq O(1) \varepsilon^{-21 / 20} e^{\frac{3 \varepsilon x}{D_{1}}} \frac{e^{-\varepsilon^{2} t / E_{0}}}{(1+t)^{\alpha / 2}} .
$$


NONLINEAR WAVE PROPAGATIONS OVER A BOLTZMANN SHOCK PROFILE 1091

One can continue the estimate of the microscopic component to obtain that $\|\mathrm{C}(x, t)\|_{L_{\xi}^{2}} \leq O(1) \varepsilon^{-21 / 20} e^{\frac{3 \varepsilon x}{D_{1}}} \frac{e^{-\varepsilon^{2} t / E_{0}}}{(1+t)^{\alpha / 2}}$, and the details are omitted as well. Similarly, one can show that for $x>0$

$$
\|\mathrm{C}(x, t)\|_{L_{\xi}^{2}} \leq O(1) \varepsilon^{-21 / 20} e^{\frac{-3 \varepsilon|x|}{D_{1}}} \frac{e^{-\varepsilon^{2} t / E_{0}}}{(1+t)^{\alpha / 2}} .
$$

Then one can proceed with the bootstrap procedure to improve the $\|\cdot\|_{L_{\xi}^{2} \text {-estimate }}$ to

$$
\|\mathrm{C}(x, t)\|_{L_{\xi, 3}^{\infty}}^{\infty} \leq O(1) \varepsilon^{-21 / 20} e^{\frac{3 \varepsilon x}{D_{1}}} \frac{e^{-\varepsilon^{2} t / E_{0}}}{(1+t)^{\alpha / 2}} .
$$

The estimates (5.102), (5.103), (5.104), and (5.105) prove this lemma for the case $x<0$. For the case $x>0$, one just needs to consider the estimates of $\mathrm{C}^{+}$and $\mathrm{D}^{+}$by the same procedure in the above. The details are omitted.

By the computations used to obtain Theorem 5.10, one has the following corollary.

Corollary 5.11. For the functions $\mathscr{U}_{\alpha}, \tilde{\mathscr{U}}_{\alpha}, \mathscr{V}_{\alpha}$ and $\tilde{\mathscr{V}}_{\alpha}$ given in (15.4), the functions $\mathbb{C}_{3}\left[\mathscr{U}_{\alpha}\right], \mathbb{C}_{3}\left[\tilde{\mathscr{U}}_{\alpha}\right], \mathbb{C}_{3}\left[\mathscr{V}_{\alpha}\right]$, and $\mathbb{C}_{3}\left[\tilde{\mathscr{V}}_{\alpha}\right]$ satisfy

$$
\begin{aligned}
& \left\|\mathbb{C}_{3}\left[\mathscr{U}_{\alpha}\right](x, t)\right\|_{L_{\xi, 3}^{\infty}} \leq O(1) \varepsilon^{-2} \frac{e^{-\frac{3 \varepsilon|x|}{D_{1}}-\frac{\varepsilon^{2} t}{E_{0}}}}{(1+t)^{\alpha / 2}} \\
& \left|\left(\mathrm{I}_{j}, \mathbb{C}_{3}\left[\mathscr{U}_{\alpha}\right](x, t)\right)\right| \leq O(1) \varepsilon^{-1} \frac{e^{-\frac{3 \varepsilon|x|}{D_{1}}-\frac{\varepsilon^{2} t}{E_{0}}}}{(1+t)^{\alpha / 2}} \text { for } j=1,2, \\
& \left\|\mathbb{C}_{3}\left[\tilde{\mathscr{U}}_{\alpha}\right](x, t)\right\|_{L_{\xi, 3}^{\infty} \leq} \leq O(1) \varepsilon^{-2} \frac{e^{-\frac{3 \varepsilon|x|}{D_{1}}}\left(e^{-\frac{\varepsilon t}{E_{0}}}+\varepsilon^{\frac{1}{2}} e^{-\frac{\varepsilon^{2} t}{E_{0}}}\right)}{(1+t)^{\alpha / 2}}, \\
& \left|\left(\mathrm{I}_{j}, \mathbb{C}_{3}\left[\tilde{\mathscr{U}}_{\alpha}\right](x, t)\right)\right| \leq O(1) \varepsilon^{-1} \frac{e^{-\frac{3 \varepsilon|x|}{D_{1}}}\left(e^{-\frac{\varepsilon t}{E_{0}}}+\varepsilon^{\frac{1}{2}} e^{-\frac{\varepsilon^{2} t}{E_{0}}}\right)}{(1+t)^{\alpha / 2}} \text { for } j=1,2, \\
& \left\|\mathbb{C}_{3}\left[\mathscr{V}_{\alpha}\right](x, t)\right\|_{L_{\xi, 3}^{\infty}} \leq O(1) \varepsilon^{-1} \frac{e^{-\frac{3 \varepsilon|x|}{D_{1}}-\frac{\varepsilon^{2} t}{E_{0}}}}{(1+t)^{\alpha / 2}}, \\
& \left|\left(\mathrm{I}_{j}, \mathbb{C}_{3}\left[\mathscr{V}_{\alpha}\right](x, t)\right)\right| \leq O(1) \frac{e^{-\frac{3 \varepsilon|x|}{D_{1}}-\frac{\varepsilon^{2} t}{E_{0}}}}{(1+t)^{\alpha / 2}} \text { for } j=1,2, \\
& \left\|\mathbb{C}_{3}\left[\tilde{\mathscr{V}}_{\alpha}\right](x, t)\right\|_{L_{\xi, 3}^{\infty}} \leq O(1) \varepsilon^{-1} \frac{e^{-\frac{3 \varepsilon|x|}{D_{1}}}\left(e^{-\frac{\varepsilon t}{E_{0}}}+\varepsilon^{\frac{1}{2}} e^{-\frac{\varepsilon^{2} t}{E_{0}}}\right)}{(1+t)^{\alpha / 2}}, \\
& \left|\left(\mathrm{I}_{j}, \mathbb{C}_{3}\left[\tilde{\mathscr{V}}_{\alpha}\right](x, t)\right)\right| \leq O(1) \frac{e^{-\frac{3 \varepsilon|x|}{D_{1}}}\left(e^{-\frac{\varepsilon t}{E_{0}}}+\varepsilon^{\frac{1}{2}} e^{-\frac{\varepsilon^{2} t}{E_{0}}}\right)}{(1+t)^{\alpha / 2}} \text { for } j=1,2 .
\end{aligned}
$$




\section{Convergence of the $\mathbb{T}-\mathbb{C}$ SCheme AND GLOBAL TIME ESTIMATE AROUND SHOCK FRONTS}

In this subsection we prove the convergence of the series $\sum_{j=1} \mathrm{q}_{j}$ defined in (4.48) and establish the global linear wave propagation structure around the shock front.

\subsection{Convergence of the $\mathbb{T}-\mathbb{C}$ scheme for the basic initial data.}

Lemma 6.1. The $\mathbb{T}-\mathbb{C}$ scheme in (4.48) satisfies the following cases for $k \geq 1$.

Case 1. $\mathrm{ER}^{c}=\mathrm{ER}^{m} \equiv 0,\left\|\mathrm{ER}^{t}\right\|_{L_{\xi}^{2}} \leq O(1) \frac{e^{-\frac{\varepsilon^{2} t}{E_{0}}-\frac{3 \varepsilon|x|}{D_{1} \mid}}}{(1+t)^{\frac{\alpha}{2}}}$.

$$
\left\|\mathfrak{r}_{k}(x, t)\right\|_{L_{\xi}^{2}} \leq \varepsilon^{k / 6} \frac{e^{-\frac{\varepsilon^{2} t}{E_{0}}}}{(1+t)^{\frac{\alpha}{2}}} e^{-\frac{3 \varepsilon|x|}{D_{1}}} .
$$

Case $2 . \mathrm{ER}^{c}=\mathrm{ER}^{m} \equiv 0,\left\|\mathrm{ER}^{t}\right\| \leq O(1) \frac{e^{-\frac{\varepsilon t}{E_{0}}-\frac{3 \varepsilon|x|}{D_{1}}}}{(1+t)^{\frac{\alpha}{2}}}$.

$$
\left\|\mathfrak{r}_{k}(x, t)\right\|_{L_{\xi}^{2}} \leq \varepsilon^{k / 6} \frac{\left(e^{-\frac{\varepsilon t}{E_{0}}}+\varepsilon^{\frac{1}{2}} e^{-\frac{\varepsilon^{2} t}{E_{0}}}\right)}{(1+t)^{\frac{\alpha}{2}}} e^{-\frac{3 \varepsilon|x|}{D_{1}}} .
$$

Case 3. $\mathrm{ER}^{t}=\mathrm{ER}^{m}=0,\left\|\mathrm{ER}^{c}\right\|_{L_{\xi}^{2}} \leq \frac{e^{-\frac{\varepsilon^{2} t}{E_{0}}-\frac{3 \varepsilon|x|}{D_{1}}}}{(1+t)^{\frac{\alpha}{2}}}$.

$$
\left\|\mathfrak{r}_{k}(x, t)\right\|_{L_{\xi}^{2}} \leq \varepsilon^{-1+k / 6} \frac{e^{-\frac{\varepsilon^{2} t}{E_{0}}}}{(1+t)^{\frac{\alpha}{2}}} e^{-\frac{3 \varepsilon|x|}{D_{1}}}
$$

Case 4. $\mathrm{ER}^{t}=\mathrm{ER}^{m}=0,\left\|\mathrm{ER}^{c}\right\|_{L_{\xi}^{2}} \leq \frac{e^{-\frac{\varepsilon t}{E_{0}}-\frac{3 \varepsilon|x|}{D_{1}}}}{(1+t)^{\frac{\alpha}{2}}}$.

$$
\left\|\mathfrak{r}_{k}(x, t)\right\|_{L_{\xi}^{2}} \leq \varepsilon^{-19 / 20} \varepsilon^{k / 6} \frac{\left(e^{-\frac{\varepsilon t}{E_{0}}}+\varepsilon^{\frac{1}{2}} e^{-\frac{\varepsilon^{2} t}{E_{0}}}\right)}{(1+t)^{\frac{\alpha}{2}}} e^{-\frac{3 \varepsilon|x|}{D_{1}}} .
$$

Case 5. $\mathrm{ER}^{t}=\mathrm{ER}^{c}=0,\left\|\mathrm{ER}^{m}\right\|_{L_{\xi, 2}^{\infty}} \leq \frac{e^{-\frac{\varepsilon^{2} t}{E_{0}}-\frac{3 \varepsilon|x|}{D_{1}}}}{(1+t)^{\frac{\alpha}{2}}}$.

$$
\left\|\mathfrak{r}_{k}(x, t)\right\|_{L_{\xi}^{2}} \leq \varepsilon^{k / 6} \frac{e^{-\frac{\varepsilon^{2} t}{E_{0}}}}{(1+t)^{\frac{\alpha}{2}}} e^{-\frac{3 \varepsilon|x|}{D_{1}}} .
$$

Case 6. $\mathrm{ER}^{t}=\mathrm{ER}^{c}=0,\left\|\mathrm{ER}^{m}\right\|_{L_{\xi, 2}^{\infty}} \leq \frac{e^{-\frac{\varepsilon t}{E_{0}}-\frac{3 \varepsilon|x|}{D_{1}}}}{(1+t)^{\frac{\alpha}{2}}}$.

$$
\left\|\mathfrak{r}_{k}(x, t)\right\|_{L_{\xi}^{2}} \leq \varepsilon^{k / 6} \frac{\left(e^{-\frac{\varepsilon t}{E_{0}}}+\varepsilon^{\frac{1}{2}} e^{-\frac{\varepsilon^{2} t}{E_{0}}}\right)}{(1+t)^{\frac{\alpha}{2}}} e^{-\frac{3 \varepsilon|x|}{D_{1}}} .
$$


Proof.

Case 1. When $k=1$, this case holds from (5.90). Assume that this case is true for $k \leq j$. Thus,

$$
\left\|\mathfrak{r}_{j}(x, t)\right\|_{L_{\xi}^{2}} \leq \varepsilon^{j / 6} \frac{e^{-\frac{\varepsilon^{2} t}{E_{0}}-\frac{3 \varepsilon|x|}{D_{1}}}}{(1+t)^{\frac{\alpha}{2}}} .
$$

Then from Lemma 5.3 and (5.90) one has

$$
\left\|\mathfrak{r}_{j+1}\right\|_{L_{\xi}^{2}}=\left\|\Re\left[\mathfrak{r}_{j}\right]\right\|_{L_{\xi}^{2}} \leq O(1) \varepsilon^{1 / 5} \varepsilon^{j / 6} \frac{e^{-\frac{\varepsilon^{2} t}{E_{0}}-\frac{3 \varepsilon|x|}{D_{1}}}}{(1+t)^{\frac{\alpha}{2}}} .
$$

This case holds for $k=j+1$. Thus, by induction this case is true for $k \in \mathbb{N}$.

The proof of Case 2 is similar to that of Case 1. The proof is omitted.

Case 3. Due to $\mathrm{ER}^{t} \equiv 0$ and the zero total macroscopic mass condition in (5.5), one has that

$$
\mathbb{R}_{3}[\mathrm{ER}]=\mathrm{ER} .
$$

Then one can apply (5.107) to yield that

$$
\left\|\mathfrak{r}_{1}[\mathrm{ER}]\right\|_{L_{\xi}^{2}} \leq \varepsilon \sum_{j=1}^{2}\left|\left(\mathrm{I}_{j}, \mathbb{C}_{3}[\mathrm{ER}]\right)\right| \leq O(1) \varepsilon^{-1+1 / 5} \frac{e^{-\frac{\varepsilon^{2} t}{E_{0}}-\frac{3 \varepsilon|x|}{D_{1}}}}{(1+t)^{\frac{\alpha}{2}}} .
$$

This case holds for $k=1$. Then by induction and from Lemma 5.3 and (5.90) one can show that this case is true for all $k \geq 1$. Furthermore, Case 4 can be obtained similarly, and its proof is omitted.

Case 5. Due to the properties $\mathrm{ER}^{t}=\mathrm{ER}^{c} \equiv 0$, one has that

$$
\mathbb{R}_{3}[\mathrm{ER}]=\mathrm{ER}, \mathbb{R}_{3}[\mathrm{ER}]^{c} \equiv 0 .
$$

Then one can apply (5.111) to yield that

$$
\left\|\mathfrak{r}_{1}[\mathrm{ER}]\right\|_{L_{\xi}^{2}} \leq \varepsilon \sum_{j=1}^{2}\left|\left(\mathrm{I}_{j}, \mathbb{C}_{3}[\mathrm{ER}]\right)\right| \leq O(1) \varepsilon^{1 / 5} \frac{e^{-\frac{\varepsilon^{2} t}{E_{0}}-\frac{3 \varepsilon|x|}{D_{1}}}}{(1+t)^{\frac{\alpha}{2}}} .
$$

Then by induction and from Lemma 5.3 and (5.90) one can show that this case is true for all $k \geq 1$. Furthermore, Case 6 can be obtained similarly, and its proof is omitted.

Theorem 6.2. Let $\mathrm{g}_{O}(x, t)$ be the approximate solution to the problem (4.5) given in (4.14), and let $\mathrm{er}_{1}(x, t)$ be the truncation error of $\mathrm{g}_{O}(x, t)$ given in (4.16). Then the series $\sum_{j=1}^{\infty} \mathfrak{r}_{j}\left[\mathrm{er}_{1}\right](x, t)$ given by the scheme in (4.48) converges for all $x \in \mathbb{R}$ and $t \geq 0$.

Proof. From Lemma 4.2, the function er ${ }_{1}$ can be decomposed into the form

$$
\operatorname{er}_{1}(x, t)=\varepsilon^{2}\left(\mathscr{I}_{2}(x, t)+\mathscr{U}_{2}(x, t)\right)+\varepsilon^{\mathscr{V}_{2}}+\varepsilon^{\frac{1}{2}} \tilde{\mathscr{V}}_{2}(x, t)+\frac{d_{0}^{\prime}(t)}{\left(\mathrm{I}_{3},\left(\mathrm{M}_{+}-\mathrm{M}_{-}\right) / \mathrm{M}_{-}^{\frac{1}{2}}\right)} \varphi^{\prime}(x)
$$


Thus, this, Theorem 5.10, and Corollary 5.11 together result in

$$
\begin{array}{r}
\mathfrak{r}_{1}(x, t)=\varepsilon \sum_{j=1}^{2}\left(\mathrm{I}_{j}, \mathbb{C}_{3}\left[\mathbb{R}_{3}\left[\varepsilon^{2}\left(\mathscr{I}_{2}(x, t)+\mathscr{U}_{2}(x, t)\right)+\varepsilon^{\mathscr{V}_{2}}+\varepsilon^{\frac{1}{2}} \tilde{\mathscr{V}}_{2}(x, t)\right]\right]\right) \mathrm{r}_{j} \\
=\varepsilon^{3} \sum_{j=1}^{2}\left(\mathrm{I}_{j}, \mathbb{C}_{3}\left[\mathbb{R}_{3}\left[\mathscr{I}_{2}(x, t)\right]\right]\right) \mathrm{r}_{j} \\
+\varepsilon^{3} \sum_{j=1}^{2}\left(\mathrm{I}_{j}, \mathbb{C}_{3}\left[\mathscr{U}_{2}(x, t)\right]\right) \mathrm{r}_{j}+\varepsilon^{2} \sum_{j=1}^{2}\left(\mathrm{I}_{j}, \mathbb{C}_{3}\left[\mathscr{\mathscr { V }}_{2}(x, t)\right]\right) \mathrm{r}_{j}+\varepsilon^{\frac{3}{2}} \sum_{j=1}^{2}\left(\mathrm{I}_{j}, \mathbb{C}_{3}\left[\tilde{\mathscr{V}}_{2}(x, t)\right]\right) \mathrm{r}_{j} \\
=O(1)\left(\varepsilon^{2} \mathscr{I}_{2}(x, t)+\varepsilon^{\frac{3}{2}} \tilde{\mathscr{I}}_{2}\right) .
\end{array}
$$

Then from this, (6.1), and (6.2) it follows for $k \geq 2$ that

$$
\mathfrak{r}_{k}=O(1) k \varepsilon^{2+\frac{(k-1)}{6}} \mathscr{I}_{2}+O(1) \varepsilon^{\frac{3}{2}+\frac{(k-1)}{6}} \tilde{\mathscr{I}}_{2} .
$$

This gives rise to the convergence of the $\mathbb{T}-\mathbb{C}$ scheme.

Proposition 6.3. For the truncation error $\mathrm{er}_{1}$ given in Theorem 6.2, the local wave front tracing function $\mathbb{D}\left[\mathrm{er}_{1}\right]$ satisfies

$$
\left\|\sum_{j=1}^{\infty} \mathbb{D}\left[\mathfrak{r}_{j}\right](x, t)\right\|_{L_{\xi}^{2}} \leq O(1) \varepsilon^{19 / 20} \frac{e^{-\varepsilon^{2} t / E_{0}}}{(1+t)} e^{\frac{-4 \varepsilon|x|}{D_{0}}} .
$$

Proof. Due to (4.49) and (6.1), the solution $g$ of (4.5) can be expressed as

$$
\mathrm{g}(x, t)=\mathrm{g}_{O}(x, t)+\sum_{j=0}^{\infty}\left(\mathbb{T}+\mathbb{C}_{3} \circ \mathbb{R}_{3}\right)\left[\mathfrak{r}_{j}\right](x, t)+\ell(t) \varphi^{\prime}(x),
$$

where $\ell(t) \varphi^{\prime}(x) \equiv \sum_{j=0} \mathbb{D}\left[\mathfrak{r}_{j}\right]$. Furthermore, from (4.20), (4.49), (5.35), (6.1), and Theorem 6.2, one has the regularity estimate on the function $\ell(t)$ :

$$
\sup _{t \geq 0} \frac{\left|\ell^{\prime}(t)\right|}{\frac{e^{-\varepsilon^{2} t / E_{0}}}{(1+t)}} \leq O(1) \varepsilon^{19 / 20} .
$$

This regularity property on $\ell(t)$ yields that

$$
\lim _{t \rightarrow \infty} \ell(t) \text { exists. }
$$

Next, we continue to show that $\lim _{t \rightarrow \infty} \ell(t)=0$.

Denote $\mathrm{j}(x, t) \equiv \mathrm{g}(x, t)-\mathrm{g}_{O}^{-}(x, t)$ where $\mathrm{g}_{O}^{-}(x, t)$ is given in (4.6). The function j satisfies

$$
\left\{\begin{array}{l}
\partial_{t} \mathrm{j}+\xi^{1} \partial_{x} \mathrm{j}-\mathrm{L}_{\varphi} \mathrm{j}=-\left(\mathrm{L}_{-}-\mathrm{L}_{\varphi}\right) \mathrm{g}_{O}^{-} \\
\mathrm{j}(x, 0)=0
\end{array}\right.
$$

Hence,

$$
\mathrm{j}(x, t)=\left(\mathrm{g}_{O}-\mathrm{g}_{O}^{-}+\sum_{k=0}^{\infty}\left(\mathbb{T}+\mathbb{C}_{3} \circ \mathbb{R}_{3}\right)\left[\mathfrak{r}_{k}\right]\right)(x, t)+\ell(t) \varphi^{\prime}(x) .
$$


From the estimate in (4.8), one has the integrable property of the function $\left\|\left(\mathrm{L}_{-}-\mathrm{L}_{\varphi}\right) \mathrm{g}_{O}^{-}(x, t)\right\|_{L_{\xi}^{2}}^{2}$ over the domain $(x, t) \in \mathbb{R} \times \mathbb{R}_{+}$:

$$
\begin{aligned}
& \text { (6.20) } \quad \int_{0}^{\infty} \int_{\mathbb{R}}\left\|\left(\mathrm{L}_{-}-\mathrm{L}_{\varphi}\right) \mathrm{g}_{O}^{-}(y, s)\right\|_{L_{\xi}^{2}}^{2} d y d s \\
& \leq O(1) \varepsilon^{2} \int_{0}^{\infty} \int_{\mathbb{R}}\left(\sum_{j=1}^{2} \frac{e^{-\frac{\left(y-\lambda_{j}^{-} s\right)^{2}}{D_{0}(s+1)}}}{(1+s)}+\frac{e^{-\frac{(y-\varepsilon s)^{2}}{D_{0}(s+1)}}}{(1+s)^{2}}\right) \frac{e^{4 \varepsilon y / D_{1}}}{e^{4 \varepsilon y / D_{1}}+e^{-4 \varepsilon y / D_{1}}} d y d s<\infty .
\end{aligned}
$$

Then one can consider the anti-derivative variable

$$
\mathrm{w}(x, t)=\int_{-\infty}^{x} \mathrm{j}(r, t) d r
$$

and the macro-micro decomposition

$$
\left\{\begin{array}{l}
\mathrm{w}_{0} \equiv \mathscr{P}_{0} \mathrm{w}, \mathrm{w}_{1} \equiv \mathscr{P}_{1} \mathrm{w}, \\
\mathrm{j}_{0}=\mathscr{P}_{0} \mathrm{j}, \quad \mathrm{j}_{1}=\mathscr{P}_{1} \mathrm{j} .
\end{array}\right.
$$

Since the image $\left(\mathrm{L}_{\varphi}-\mathrm{L}_{-}\right) \mathrm{g}_{O}^{-}$is purely microscopic, one has

$$
\begin{aligned}
& \partial_{t} \mathrm{w}_{0}+\mathscr{P}_{0} \xi^{1}\left(\mathscr{P}_{0} \partial_{x} \mathrm{w}_{0}+\mathrm{j}_{1}\right)=0, \\
& \partial_{t} \mathrm{j}+\xi^{1} \partial_{x} \mathrm{j}-\mathrm{L}_{\varphi} \mathrm{j}=-\left(\mathrm{L}_{-}-\mathrm{L}_{\varphi}\right) \mathrm{g}_{O}^{-} .
\end{aligned}
$$

This leads to the energy estimate for some $K_{0} \gg 1$

$$
\begin{aligned}
0=\int_{0}^{\infty} \int_{\mathbb{R}}\left\langle\mathrm{w}_{0}, \partial_{t} \mathrm{w}_{0}+\right. & \left.\mathscr{P}_{0} \xi^{1}\left(\mathscr{P}_{0} \partial_{x} \mathrm{w}_{0}+\mathrm{j}_{1}\right)\right\rangle \\
& +K_{0}\left(\mathrm{j}, \partial_{t} \mathrm{j}+\xi^{1} \partial_{x} \mathrm{j}-\mathrm{L}_{\varphi} \mathrm{j}+\left(\mathrm{L}_{-}-\mathrm{L}_{\varphi}\right) \mathrm{g}_{O}^{-}\right) d x d t
\end{aligned}
$$

where

$$
\langle\mathrm{F}(x, t), \mathrm{J}(x, t)\rangle \equiv \int_{\mathbb{R}^{3}} \frac{\mathrm{F}(x, t, \xi) \mathrm{J}(x, t, \xi) \mathrm{M}_{-}(\xi)}{\mathrm{M}_{[1, \bar{u}(x), \overline{\boldsymbol{T}}(x)]}(\xi)} d \xi .
$$

Then by the energy method in 12 it follows from (6.23) that

$$
\begin{aligned}
\int_{0}^{\infty} \int_{\mathbb{R}} \varepsilon^{2}\left\langle\mathrm{w}_{0}(x, t), \mathrm{w}_{0}(x, t)\right\rangle e^{-\varepsilon|x| / D_{1}} d x d t & \\
+\int_{0}^{\infty} \int_{\mathbb{R}}\left(\left\langle\mathscr{P}_{1} \xi^{1} \partial_{x} \mathrm{w}_{0}(x, t),\right.\right. & \left.\left.\mathscr{P}_{1} \xi^{1} \partial_{x} \mathrm{w}_{0}(x, t)\right\rangle+K_{0}\left(\mathscr{P}_{1} \mathrm{j}(x, t), \mathscr{P}_{1} \mathrm{j}(x, t)\right)\right) d x d t \\
& \leq O(1) \int_{0}^{\infty} \int_{\mathbb{R}}\left\|\left(\mathrm{L}_{-}-\mathrm{L}_{\varphi}\right) \mathrm{g}_{O}^{-}(x, t)\right\|_{L_{\xi}^{2}}^{2} d x d t<\infty
\end{aligned}
$$

Due to the facts that

$$
\lim _{t \rightarrow \infty}\left\|\mathrm{g}_{O}\right\|_{L_{x}^{\infty}\left(L_{\xi}^{2}\right)}+\lim _{t \rightarrow \infty}\left\|\sum_{j=1}\left(\mathbb{T}+\mathbb{C}_{3} \circ \mathbb{R}_{3}\right)\left[\mathrm{TC}_{j}\left[\mathrm{er}_{1}\right]\right]\right\|_{L_{x}^{\infty}\left(L_{\xi}^{2}\right)}=0
$$

and (6.19), one can conclude that

$$
\lim _{t \rightarrow \infty} \ell(t)=0
$$

otherwise

which is a contraction to (6.24).

$$
\int_{0}^{\infty} \int_{\mathbb{R}}\left(\mathscr{P}_{1} \mathrm{j}, \mathscr{P}_{1 \mathrm{j}}\right) d x d t=\infty,
$$


Formulas (6.25) and (6.16) result in

$$
|\ell(t)| \leq O(1) \varepsilon^{-21 / 20} \frac{e^{-\varepsilon^{2} t / E_{0}}}{(1+t)} .
$$

This concludes the proof of the proposition.

Theorem 6.4. The solution $\mathrm{g}(x, t)$ of (4.5) satisfies

$$
\begin{aligned}
& \|\mathrm{g}(x, t)\|_{L_{\xi, 3}^{\infty}} \\
& \leq O(1)\left[\chi_{-}(x)\left(\sum_{j=1}^{2} \frac{e^{-\frac{\left(x-\lambda_{j}^{-} t\right)^{2}}{C_{0}(t+1)}}}{\sqrt{(t+1)}}+\frac{e^{-\frac{(x-\varepsilon t)^{2}}{C_{0}(t+1)}}}{(t+1)}\right)\right. \\
& \left.+\chi_{+}(x)\left(\sum_{j=1}^{2} \frac{e^{-\frac{\left(x-\lambda_{j}^{+} t\right)^{2}}{C_{0}(t+1)}}}{\sqrt{(t+1)}}+\frac{e^{-\frac{(x+\varepsilon t)^{2}}{C_{0}(t+1)}}}{(t+1)}\right)\right] \\
& +O(1) \varepsilon^{2} \mathscr{A}_{2}(x, t)+O(1) \varepsilon^{19 / 20} e^{\frac{-3 \varepsilon|x|}{D_{1}}} \frac{e^{-|\varepsilon|^{2} t / E_{0}}}{(1+t)},
\end{aligned}
$$

where

$$
\begin{aligned}
& \text { (6.28) } \mathscr{A}_{2}(x, t)
\end{aligned}
$$

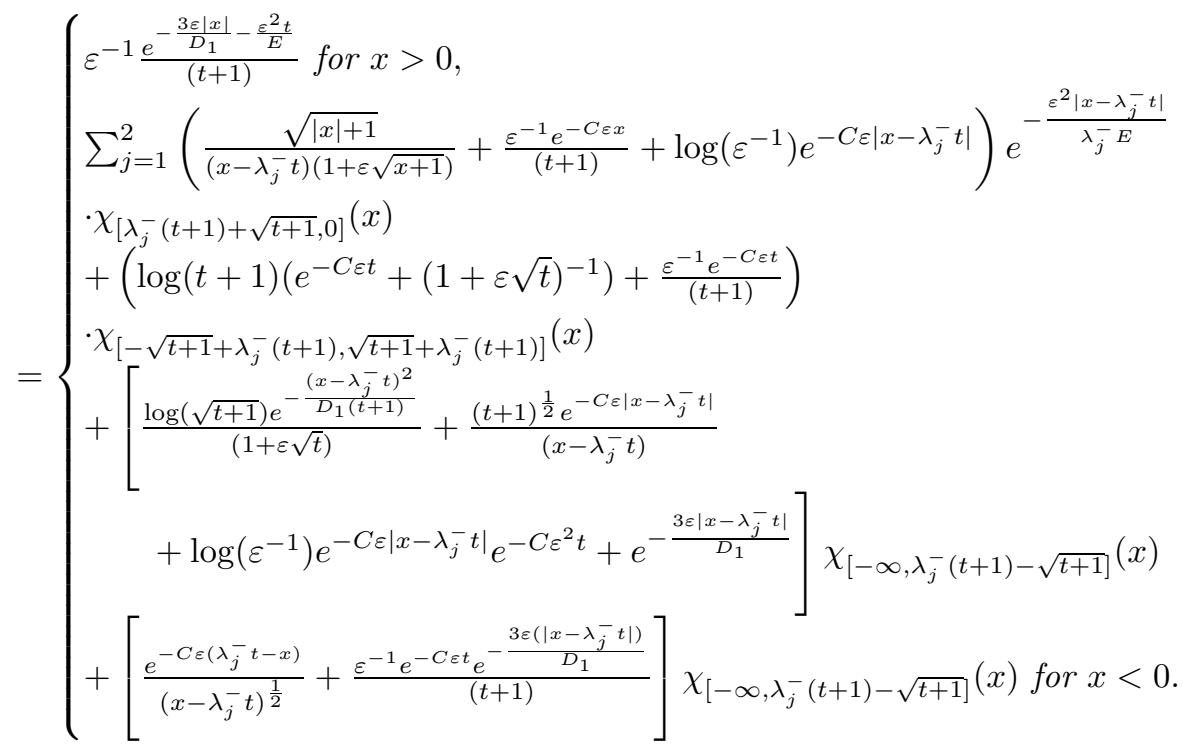

Proof. From (6.11), (6.12), Lemma 6.1, (4.20), (4.19), and (5.6) all together with $\alpha=2$, one applies (6.1) to obtain that

$$
\left\|\sum_{j=0}^{\infty} \mathbb{T}\left[\mathfrak{r}_{j}\left[\mathrm{er}_{1}\right]\right](x, t)\right\|_{L_{\xi, 3}^{\infty}}
$$

$$
=O(1)\left\|\mathbb{T}\left[\mathrm{er}_{1}\right](x, t)\right\|_{L_{\xi, 3}^{\infty}}=O(1) \varepsilon^{2} \sum_{l=1}^{2} \mathbb{K}^{2,1 ; \frac{\varepsilon^{2}}{E_{0}}}\left(x, t ; \lambda_{l}^{-}, D_{0}, E_{0}\right) \leq O(1) \varepsilon^{2} \mathscr{A}_{2}(x, t) .
$$


From (6.11), (6.12), Lemma 6.1, (4.20), (4.19), and (5.6), (5.9) all together with $\alpha=2$, one applies (5.92) to obtain that

$$
\left\|\sum_{j=1}^{\infty} \mathbb{C}_{3}\left[\mathbb{R}_{3}\left[\mathfrak{r}_{j}\left[\mathrm{er}_{1}\right]\right]\right](x, t)\right\|_{L_{\xi, 3}^{\infty}}=O(1) \varepsilon^{19 / 20} e^{-\frac{3 \varepsilon|x|}{D_{1}}} \frac{e^{-|\varepsilon|^{2} t / E_{0}}}{(1+t)} .
$$

Then from (6.15), (4.14), (6.29), (6.30), and (6.14) all together we conclude (6.27).

Lemma 6.5. Suppose that the functions $a(x)$ and $b(x)$ satisfy

$$
\left\{\begin{array}{l}
\|a\|_{L_{x}^{\infty}},\|b\|_{L_{x}^{\infty}} \leq O(1), \\
\operatorname{supp}(a) \subset[-1,1],|b(x)| \leq O(1) \varepsilon e^{-\frac{3 \varepsilon}{D_{0}}|x|} \text { for }|x| \geq 1, \\
\int_{\mathbb{R}} a(x) d x=0, \int_{\mathbb{R}} b(x) d x=0 .
\end{array}\right.
$$

Then for the operator $\mathbb{I}$ given in (4.50) the functions $\mathbb{I}_{3}\left[a(\cdot) \mathrm{r}_{3}\right](x, t)$ and $\mathbb{I}_{3}\left[b(\cdot) \mathrm{r}_{3}\right](x, t)$ satisfy

$$
\left\{\begin{array}{l}
\left|\left(\mathrm{I}_{i}, \mathbb{I}_{3}\left[a(\cdot) \mathrm{r}_{3}\right](x, t)\right)\right|,\left|\left(\mathrm{I}_{j}, \mathbb{I}_{3}\left[b(\cdot) \mathbf{r}_{3}\right](x, t)\right)\right| \leq O(1) \frac{e^{-\frac{3 \varepsilon|x|}{D_{1}}-\frac{\varepsilon^{2} t}{E_{1}}}}{(1+t)} \\
\text { for } i=1,2,3, j=1,2, \\
\left|\left(\mathrm{I}_{3}, \mathbb{I}_{3}\left[b(\cdot) \mathrm{r}_{3}\right](x, t)\right)\right| \leq O(1) \frac{e^{-\frac{\varepsilon|x|}{D_{1}}-\frac{\varepsilon^{2} t}{E_{1}}}}{(1+t)^{1 / 2}} .
\end{array}\right.
$$

This lemma can be obtained by the energy method and the energy-pointwise estimate for $\mathbb{C}_{3}\left[\mathbb{R}_{3}[E R]\right]$ in Subsections [5.3, 5.4. The details is omitted.

Theorem 6.6. The solution $\mathrm{g}_{I}(x, t)$ of (4.51) satisfies

$$
\left\{\begin{array}{l}
\left\|\mathrm{g}_{I}(x, t)-c^{*} \varphi^{\prime}(x)\right\|_{L_{\xi, 3}^{\infty}} \leq O(1) \frac{e^{-3 \frac{\varepsilon|x|}{D_{1}}-\frac{\varepsilon^{2} t}{E_{0}}}}{(1+t)^{\frac{1}{2}}}+O(1) \varepsilon \mathscr{A}_{2}(x, t), \\
c^{*}=\int_{\mathbb{R}} c\left(x ; y_{0}\right) d x /\left(\mathrm{I}_{3},\left(\mathrm{M}_{+}-\mathrm{M}_{-}\right) / \sqrt{\mathrm{M}_{-}}\right),
\end{array}\right.
$$

and the solution $\mathrm{g}_{I I}(x, t)$ of (4.52) satisfies

$$
\left\|\mathrm{g}_{I I}(x, t)\right\|_{L_{\xi, 3}^{\infty}} \leq O(1) \frac{e^{-\frac{3 \varepsilon|x|}{D_{1}}-\frac{\varepsilon^{2} t}{E_{0}}}}{(1+t)}+O(1) \varepsilon \mathscr{A}_{2}(x, t)
$$

Proof. From the estimate of $\mathbb{I}_{3}\left[b r_{3}\right]$ in (6.31), one has that

$$
\left\{\begin{aligned}
&\left|\left(\mathrm{I}_{j}, \mathrm{q}_{0}(x, t)\right)\right|=\left|\left(\mathrm{I}_{j}, \mathbb{I}_{3}\left[c(\cdot, 0) \mathrm{r}_{3}-c^{*} \varphi^{\prime}(\cdot)\right](x, t)\right)\right| \leq O(1) \frac{e^{-\frac{\varepsilon|x|}{D_{1}}-\frac{\varepsilon^{2} t}{E_{0}}}}{(1+t)} \text { for } j=1,2, \\
&\left|\left(\mathrm{I}_{3}, \mathrm{q}_{0}(x, t)\right)\right|=\left|\left(\mathrm{I}_{3}, \mathbb{I}_{3}\left[c(\cdot, 0) \mathrm{r}_{3}-c^{*} \varphi^{\prime}(\cdot)\right](x, t)\right)\right| \leq O(1) \frac{e^{-3 \frac{\varepsilon|x|}{D_{1}}-\frac{\varepsilon^{2} t}{E_{0}}}}{(1+t)^{1 / 2}} .
\end{aligned}\right.
$$

From (6.34), one has

$$
\left\|\mathfrak{r}_{0}(x, t)\right\|_{L_{\xi}^{2}} \leq O(1) \varepsilon \frac{e^{-3 \frac{\varepsilon|x|}{D_{1}}-\frac{\varepsilon^{2} t}{E_{0}}}}{(1+t)}
$$


Then from Lemma 5.3 (5.102), (5.90) and by induction, one has that

$$
\left\|\mathfrak{r}_{k}(x, t)\right\|_{L_{\xi}^{2}} \leq O(1) \varepsilon^{1+\frac{k}{10}} \frac{e^{-3 \frac{\varepsilon|x|}{D_{1}}-\frac{\varepsilon^{2} t}{E_{0}}}}{(1+t)} .
$$

This shows the convergence of the series $\sum_{j=0}^{\infty} \mathrm{q}_{j}(x, t)$ pointwise and gives rise to

$$
\mathrm{g}_{I}(x, t)=c^{*} \varphi^{\prime}(x)+\sum_{j=0}^{\infty} \mathbf{q}_{j}(x, t)=c^{*} \varphi^{\prime}(x)+O(1)\left(\mathbf{q}_{0}(x, t)+\mathbb{T}\left[\mathfrak{r}_{0}\right]\right)(x, t) .
$$

From this, (6.36), and Lemma 5.1, this theorem follows.

For the solution $\mathrm{g}_{I I}(x, t)$, its initial data is purely microscopic so that the coefficient $c^{*}=0$. Then one can apply the estimate of $\mathbb{I}_{3}\left[a r_{3}\right]$ in (6.31) to yield that the $\mathfrak{r}_{0}$ for $\mathrm{g}_{I I}(x, t)$ satisfies

$$
\left\|\mathfrak{r}_{0}(x, t)\right\|_{L_{\xi}^{2}} \leq O(1) \varepsilon \frac{e^{-3 \frac{\varepsilon|x|}{D_{1}}-\frac{\varepsilon^{2} t}{E_{0}}}}{(1+t)} .
$$

Then one follows the same argument to conclude the estimate for $\mathrm{g}_{I I}(x, t)$. The theorem follows.

In the estimate of (6.33), the function $\mathscr{A}_{2}(x, t)$ does not decay fast enough around the position $x=\lambda_{j}^{-} t, j=1,2$. The solution $\mathrm{g}_{I I}(x, t)$ can be improved as follows.

Corollary 6.7 (Improved Rate). The solution $\mathrm{g}_{I I}(x, t)$ of (4.52) satisfies for $x \leq$ $-\sqrt{\frac{5 \boldsymbol{T}_{-}}{3}} / 2$

$$
\left\|\mathrm{g}_{I I}(x, t)\right\|_{L_{\xi, 3}^{\infty}} \leq O(1) \frac{\mathscr{A}_{2}(x, t)}{\sqrt{1+t}} .
$$

Proof. From (6.33) one has

$$
\left\|\mathrm{g}_{I I}(x, t)\right\|_{L_{\xi, 3}^{\infty} \leq} \leq O(1)\left(\frac{e^{-\frac{3 \varepsilon|x|}{D_{1}}-\frac{\varepsilon^{2} t}{E_{0}}}}{(1+t)}+\varepsilon \mathscr{A}_{2}(x, t)\right) .
$$

This yields that

$$
\left\|\left(\mathrm{L}_{\varphi}-\mathrm{L}_{-}\right) \mathrm{g}_{I I}(x, t)\right\|_{L_{\xi, 2}^{\infty}} \leq O(1) \varepsilon \frac{e^{-\frac{3 \varepsilon|x|}{D_{1}}-\frac{\varepsilon^{2} t}{E_{0}}}}{(1+t)} .
$$

From this, one can have the representation of $\mathrm{g}_{I I}(x, t)$

$$
\begin{aligned}
\mathrm{g}_{I I}(x, t)=\int_{\mathbb{R}} \mathbb{G}_{-}(x-y, t) & \mathrm{g}_{I I}(y, 0) d y \\
& +\int_{0}^{t} \int_{\mathbb{R}} \mathbb{G}_{-}(x-y, t-s)\left(\mathrm{L}_{\varphi}-\mathrm{L}_{-}\right) \mathrm{g}_{I I}(y, s) d y d s .
\end{aligned}
$$

This, (6.41), and (A.6) with $(\alpha, \beta)=(2,2)$ (due to the fact that $\left(\mathrm{L}_{\varphi}-\mathrm{L}_{-}\right) \mathrm{g}_{I I}$ is 
purely microscopic) yield that

$$
\left\|\mathrm{g}_{I I}(x, t)\right\|_{L_{\xi, 3}^{\infty} \leq} \leq O(1)\left(\sum_{j=1}^{3} \frac{e^{-\frac{\left(x-\lambda_{j}^{-} t\right)^{2}}{D_{0}(t+1)}}}{(t+1)}+\sum_{j=1}^{2} \mathbb{K}^{2,2 ; \frac{\varepsilon^{2}}{E_{0}}}\left(x, t ; \lambda_{j}^{-}, D_{0}, E_{0}\right)\right)
$$$$
+\frac{e^{-2 \frac{\varepsilon|x|}{D_{0}}-\frac{\varepsilon^{2} t}{E_{0}}}}{\varepsilon(1+t)}
$$$$
\leq O(1)\left(\sum_{j=1}^{3} \frac{e^{-\frac{(x-\lambda \bar{j} t)^{2}}{D_{0}(t+1)}}}{(t+1)} \frac{e^{-2 \frac{\varepsilon|x|}{D_{0}}-\frac{\varepsilon^{2} t}{E_{0}}}}{\varepsilon(1+t)}\right)+O(1) \frac{\varepsilon^{-\frac{1}{2}}}{(t+1)} e^{-\frac{4 \varepsilon|x|}{D}-\frac{\varepsilon^{2} t}{E}} \chi_{[0, \infty)}
$$$$
+O(1) \sum_{j=1}^{2}\left(\frac{1}{\left(\lambda_{j}^{-} t-x\right)(1+\varepsilon \sqrt{x+1})}+\frac{\varepsilon^{-\frac{1}{2}} e^{-C \varepsilon x}}{(t+1)}+\frac{\log \left(\varepsilon^{-1}\right) e^{-C \varepsilon\left|x-\lambda_{j}^{-} t\right|}}{(t+1)^{\frac{1}{2}}}\right)
$$

$$
\begin{gathered}
\cdot e^{-\frac{\varepsilon^{2}\left|x-\lambda_{j}^{-} t\right|}{\lambda_{j}^{-} E}} \chi_{\left[\lambda_{j}^{-}(t+1)-\sqrt{t+1}, 0\right)}\left(\frac{\log (t+1) e^{-C \varepsilon t}+\log (t+1)(1+\varepsilon \sqrt{t})^{-1}}{(t+1)^{\frac{1}{2}}}+\frac{(t+1)^{\frac{1}{2}} e^{-C \varepsilon t}}{(t+1)}\right) \\
+\chi_{\left[\lambda_{-}^{-}(t+1)-\sqrt{t+1}, \lambda^{-}(t+1)+\sqrt{t+1}\right.}
\end{gathered}
$$

$$
\cdot \chi_{\left[\lambda_{j}^{-}(t+1)-\sqrt{t+1}, \lambda_{j}^{-}(t+1)+\sqrt{t+1}\right]}
$$

$+O(1)(t+1)^{-\frac{1}{2}}\left[\frac{\log (t+1) e^{-\frac{\left(x-\lambda_{j}^{-} t\right)^{2}}{D(t+1)}}}{(1+\varepsilon \sqrt{t})}\right.$

$$
\left.+\frac{(t+1)^{\frac{1}{2}} e^{-C \varepsilon\left|x-\lambda_{j}^{-} t\right|}}{\left(x-\lambda_{j}^{-} t\right)}+\log \left(\varepsilon^{-1}\right) e^{-C \varepsilon\left|x-\lambda_{j}^{-} t\right|} e^{-C \varepsilon^{2} t}+e^{-\frac{4 \varepsilon\left(x-\lambda_{j}^{-} t\right)}{D}}\right]
$$

$$
\cdot \chi_{\left(-\infty, \lambda_{j}^{-}(t+1)-\sqrt{t+1}\right]}
$$

$+O(1)\left[\frac{e^{-C \varepsilon\left(x-\lambda_{j}^{-} t\right)}}{\left(x-\lambda_{j}^{-} t\right)^{\frac{3}{2}}}+\frac{\left(\left(\varepsilon^{-\frac{1}{2}}\right)+(t+1)^{\frac{1}{2}}\right) e^{-C \varepsilon t} e^{-\frac{4 \varepsilon\left(x-\lambda_{j}^{-} t\right)}{D}}}{(t+1)}\right] \chi_{\left(-\infty, \lambda_{j}^{-}(t+1)-\sqrt{t+1}\right]}$.

This concludes the proof of (6.39).

\section{A Mild Generalization of Initial Data}

One replaces the initial datum of (4.5), (4.51), and (4.52) by the following:

$$
\left\{\begin{array}{l}
\left|a\left(x ; y_{0}\right)\right|,\left|b\left(x ; y_{0}\right)\right|,\left|c\left(x ; y_{0}\right)\right| \leq \varepsilon e^{-\frac{3 \varepsilon\left|x-y_{0}\right|}{D_{1}}} \\
\left\|\mathrm{~d}\left(x ; y_{0}\right)\right\|_{L_{\xi, 3}^{\infty}} \leq \varepsilon e^{-\frac{3 \varepsilon\left|x-y_{0}\right|}{D_{1}}} \\
y_{0}=0
\end{array}\right.
$$

The analysis in obtaining Theorems 6.4 and 6.6 can be applied to yield the following corollary: 
Corollary 6.8. The solution of the initial value problem $\left(\partial_{t}+\xi^{1} \partial_{x}-\mathrm{L}_{\varphi}\right) \mathrm{h}=0$ satisfies the following cases:

Case $\mathrm{h}^{c}(x, 0)=\mathrm{h}^{m}(x, 0)=0,\left\|\mathrm{~h}^{t}(x, 0)\right\|_{L_{\xi}^{2}} \leq O(1) \varepsilon e^{-\frac{\varepsilon|x|}{D_{0}}}$.

For $t \in\left(0, \varepsilon^{-2}\right)$,

(6.45) $\|\mathrm{h}(x, t)\|_{L_{\xi, 3}^{\infty}}$

$$
\leq O(1) \sum_{j=1}^{2}\left(\chi_{-}(x) \varepsilon e^{-\varepsilon \frac{\left|x-\lambda_{j}^{-} t\right|}{D_{1}}}+\chi_{+}(x) \varepsilon e^{-\varepsilon \frac{\left|x-\lambda_{j}^{+} t\right|}{D_{1}}}\right)+\frac{\varepsilon e^{-\frac{\varepsilon|x|}{D_{1}}}}{\sqrt{(t+1)}}+\varepsilon^{2} \mathscr{A}_{2}(x, t) .
$$

For $t \in\left(\varepsilon^{-2}, \infty\right)$,

$$
\begin{array}{r}
\|\mathrm{h}(x, t)\|_{L_{\xi, 3}^{\infty}} \leq O(1) \chi_{-}(x)\left(\sum_{j=1}^{2}\left(\frac{e^{-\frac{\left(x-\lambda_{j}^{-} t\right)^{2}}{D_{0}(t+1)}}}{\sqrt{(t+1)}}+\varepsilon e^{-\varepsilon \frac{\left|x-\lambda_{j}^{-} t\right|}{D_{1}}}\left(1-\chi_{\left[\lambda_{j}^{-} t-\sqrt{t}, \lambda_{j}^{-} t+\sqrt{t}\right]}\right)\right)\right. \\
\left.+\frac{e^{-\frac{(x-\varepsilon t)^{2}}{D_{0}(t+1)}}}{(t+1)}\right)+O(1) \chi_{+}(x)\left(\sum_{j=1}^{2}\left(\frac{e^{-\frac{\left(x-\lambda_{j}^{+} t\right)^{2}}{D_{0}(t+1)}}}{\sqrt{(t+1)}}+\varepsilon e^{-\varepsilon \frac{\left|x-\lambda_{j}^{-} t\right|}{D_{1}}}\left(1-\chi_{\left[\lambda_{j}^{+} t-\sqrt{t}, \lambda_{j}^{+} t+\sqrt{t}\right]}\right)\right)\right. \\
\left.+\frac{e^{-\frac{(x+\varepsilon t)^{2}}{D_{0}(t+1)}}}{(t+1)}\right)+\varepsilon^{2} \mathscr{A}_{2}(x, t) .
\end{array}
$$

Case $\mathrm{h}^{t}(x, t)=\mathrm{h}^{c}(x, 0)=0,\left\|\mathrm{~h}^{m}(x, 0)\right\|_{L_{\xi, 3}^{\infty}} \leq O(1) \varepsilon e^{-\frac{\varepsilon|x|}{D_{0}}}$.

For $t \in\left(0, \varepsilon^{-2}\right)$,

(6.47) $\|\mathrm{h}(x, t)\|_{L_{\xi, 3}^{\infty}}$

$$
\leq O(1)\left(\frac{1}{\sqrt{1+t}} \sum_{j=1}^{2}\left(\chi_{-}(x) \varepsilon e^{-\varepsilon \frac{\left|x-\lambda_{j}^{-} t\right|}{D_{1}}}+\chi_{+}(x) \varepsilon e^{-\varepsilon \frac{\left|x-\lambda_{j}^{+} t\right|}{D_{1}}}\right)+\frac{\varepsilon e^{-\varepsilon \frac{|x|}{D_{1}}}}{\sqrt{1+t}}+\varepsilon^{2} \mathscr{A}_{2}(x, t)\right) .
$$

For $t \in\left(\varepsilon^{-2}, \infty\right)$,

(6.48) $\quad\|\mathrm{h}(x, t)\|_{L_{\xi, 3}^{\infty}}$

$$
\begin{gathered}
\leq \chi_{-}(x)\left(\frac{O(1)}{\sqrt{(t+1)}}\left[\sum_{j=1}^{2} \frac{e^{-\frac{\left(x-\lambda_{j}^{-} t\right)^{2}}{D_{0}(t+1)}}}{\sqrt{(t+1)}}+\varepsilon e^{-\varepsilon \frac{\left|x-\lambda_{j}^{-} t\right|}{D_{1}}}\left(1-\chi_{\left[\lambda_{j}^{-} t-\sqrt{t}, \lambda_{j}^{-} t+\sqrt{t}\right]}\right)\right]\right. \\
\left.+\frac{e^{-\frac{(x-\varepsilon t)^{2}}{D_{0}(t+1)}}}{(t+1)}\right) \\
+\chi_{+}(x)\left(\frac{O(1)}{\sqrt{1+t}}\left[\sum_{j=1}^{2} \frac{e^{-\frac{\left(x-\lambda_{j}^{+} t\right)^{2}}{D_{0}(t+1)}}}{\sqrt{(t+1)}}+\varepsilon e^{-\frac{\left|x-\lambda_{j}^{-} t\right|}{D_{1}}}\left(1-\chi_{\left[\lambda_{j}^{+} t-\sqrt{t}, \lambda_{j}^{+} t+\sqrt{t}\right]}\right)\right]+\frac{e^{-\frac{(x+\varepsilon t)^{2}}{D_{0}(t+1)}}}{(t+1)}\right) \\
+\varepsilon^{2} \mathscr{A}_{2}(x, t) .
\end{gathered}
$$


NONLINEAR WAVE PROPAGATIONS OVER A BOLTZMANN SHOCK PROFILE 1101

6.2. A representation of solutions with center $y_{0}$ away from zero. For an initial value problem $\left(\partial_{t}+\xi^{1} \partial_{x}-\mathrm{L}_{\varphi}\right) \mathrm{h}=0$ with a given initial data $\mathrm{h}_{0}(x)$, which possesses the pointwise structure that

$$
\sup _{x \in \mathbb{R}} e^{\frac{3 \varepsilon|x|}{D_{0}}}\left\|\mathrm{~h}_{0}(x)\right\|_{L_{\xi, 3}^{\infty}}<\infty,
$$

by Corollary 6.8, the solution of 6.49 defines a semi-group $\mathbb{G}_{\varphi}^{t}$ as follows:

$$
\mathbb{G}_{\varphi}^{t}\left[\mathrm{~h}_{0}\right](x) \equiv \mathrm{h}(x, t) .
$$

\section{An Outer Approximate Function and Its Truncation Error}

For an initial value problem

$$
\left\{\begin{array}{l}
\left(\partial_{t}+\xi^{1} \partial_{x}-\mathrm{L}_{\varphi}\right) \mathrm{f}\left(x, t ; y_{0}\right)=0 \\
\mathrm{f}\left(x, 0 ; y_{0}\right) \equiv 0 \text { for }\left|x-y_{0}\right| \geq 1 \\
\left\|\mathrm{f}\left(x, 0 ; y_{0}\right)\right\|_{L_{\xi, 3}^{\infty}} \leq 1 \text { for }\left|x-y_{0}\right| \leq 1
\end{array}\right.
$$

with $y_{0}<0$ being an arbitrary negative number, the outer approximate solution $\mathrm{f}_{O}\left(x, t ; y_{0}\right)$ is defined by

$$
\mathrm{f}_{O}\left(x, t ; y_{0}\right) \equiv \int_{\mathbb{R}}\left(\chi_{-}(x) \mathbb{G}_{-}(x-y, t)+\chi_{+}(x) \mathbb{G}_{+}(x-y, t)\right) \mathrm{f}\left(y, 0 ; y_{0}\right) d y
$$

and its truncation error $\mathfrak{E}\left(x, t ; y_{0}\right)$ is defined by

$$
\mathfrak{E}\left(x, t ; y_{0}\right) \equiv-\left(\partial_{t}+\xi^{1} \partial_{x}-\mathrm{L}_{\varphi}\right) \mathrm{f}_{O}\left(x, t ; y_{0}\right) .
$$

Lemma 6.9. Suppose that $y_{0} \leq-\varepsilon^{-1}$ and $\mathrm{f}\left(x, 0 ; y_{0}\right)$ satisfies (6.57). Then the function $\mathfrak{E}\left(x, t ; y_{0}\right)$ satisfies

$$
\left\{\begin{array}{l}
\left\|\mathfrak{E}^{c}\left(x, t ; y_{0}\right)\right\|_{L_{\xi}^{2}} \leq O(1) \frac{\varepsilon^{2} e^{-\frac{\left(y_{0}+\lambda_{3}^{-} t\right)^{2}}{D_{0}(1+t)}-\frac{3 \varepsilon|x|}{D_{0}}}}{(1+t)}, \\
\left\|\mathfrak{E}^{t}\left(x, t ; y_{0}\right)\right\|_{L_{\xi}^{2}} \leq O(1) \frac{\varepsilon^{3} e^{-\frac{\left(y_{0}+\lambda_{3}^{-} t\right)^{2}}{D_{0}(1+t)}-\frac{3 \varepsilon|x|}{D_{0}}}}{(1+t)}, \\
\left\|\mathfrak{E}^{m}\left(x, t ; y_{0}\right)\right\|_{L_{\xi, 2}^{\infty}} \leq O(1) \frac{\varepsilon e^{-\frac{\left(y_{0}+\lambda_{3}^{-} t\right)^{2}}{D_{0}(1+t)}-\frac{3 \varepsilon|x|}{D_{0}}}}{(1+t)} .
\end{array}\right.
$$

Proof. From Corollary 2.11 with the improved estimates in $\|\cdot\|_{L_{\xi, 3}^{\infty}}$, one has that

$$
\left\{\begin{array}{l}
\chi_{-}^{\prime}(x)\left|\left(\mathrm{I}_{i}, \mathrm{f}_{O}\left(x, t ; y_{0}\right)\right)\right| \leq O(1) \varepsilon e^{-\frac{3 \varepsilon|x|}{D_{0}}} \frac{e^{-\frac{\left(y_{0}+\lambda_{3}^{-} t\right)^{2}}{D_{0}(t+1)}}}{(1+t)^{3 / 2}} \text { for } i=1,2, \\
\chi_{-}^{\prime}(x)\left|\left(\mathrm{I}_{3}, \mathrm{f}_{O}\left(x, t ; y_{0}\right)\right)\right| \leq O(1) \varepsilon e^{-\frac{3 \varepsilon|x|}{D_{0}}} \frac{e^{-\frac{\left(y_{0}+\lambda_{3}^{-} t\right)^{2}}{D_{0}(t+1)}}}{(1+t)} \\
\chi_{-}^{\prime}(x)\left\|\mathrm{P}_{1}^{-} \mathrm{f}_{O}\left(x, t ; y_{0}\right)\right\|_{L_{\xi, 3}^{\infty}}^{\infty} \leq O(1) \varepsilon e^{-\frac{3 \varepsilon|x|}{D_{0}}} \frac{e^{-\frac{\left(y_{0}+\lambda_{3}\right.}{D_{0}(t+1)}}}{(1+t)^{3 / 2}}
\end{array}\right.
$$

This yields the estimates of $\mathfrak{E}^{c}$ and $\mathfrak{E}^{m}$ in 6.53 . The estimate of $\mathfrak{E}^{t}$ in 6.53 is a consequence of (6.54) and (2.3), which is the second tangential property of the Hugoniot curve. 
The function $\mathfrak{E}\left(x, t ; y_{0}\right)$ satisfies the property 6 so that one can use the semi-group $\mathbb{G}_{\varphi}^{t}$ to represent the function $\mathrm{f}\left(x, t ; y_{0}\right)$ as follows:

$$
\mathrm{f}\left(x, t ; y_{0}\right)=\mathrm{f}_{O}\left(x, t ; y_{0}\right)+\int_{0}^{t} \mathbb{G}_{\varphi}^{t-s}[\mathfrak{E}(\cdot, s)](x) d s .
$$

One decomposes the function $\mathfrak{E}$ into three parts, $\mathfrak{E}=\mathfrak{E}^{t}+\mathfrak{E}^{c}+\mathfrak{E}^{m}$, so that (6.55) can be rewritten:

$$
\begin{aligned}
\mathrm{f}\left(x, t ; y_{0}\right)=\mathrm{f}_{O}\left(x, t ; y_{0}\right) & +\int_{0}^{t} \mathbb{G}_{\varphi}^{t-s}\left[\mathfrak{E}^{t}(\cdot, s)\right](x) d s \\
& +\int_{0}^{t} \mathbb{G}_{\varphi}^{t-s}\left[\mathfrak{E}^{c}(\cdot, s)\right](x) d s+\int_{0}^{t} \mathbb{G}_{\varphi}^{t-s}\left[\mathfrak{E}^{m}(\cdot, s)\right](x) d s .
\end{aligned}
$$

For the purpose of studying the nonlinear coupling of the Boltzmann equation, we only are interested in the purely microscopic initial data for (6.51), i.e.

$$
\mathrm{P}_{0}^{-} \mathrm{f}\left(x, 0 ; y_{0}\right) \equiv 0 .
$$

Lemma 6.10. Assume the initial data $\mathrm{f}\left(x, t ; y_{0}\right)$ satisfying (6.57). Then the truncation error $\mathfrak{E}(x, t)$ satisfies

$$
\int_{\mathbb{R}} \mathfrak{E}^{t}\left(x, t ; y_{0}\right)+\mathfrak{E}^{c}\left(x, t ; y_{0}\right) d x=0 \text { for } t \geq 0 .
$$

This lemma is a consequence of the macroscopic conservation laws for the Boltzmann equation. The proof is omitted.

From Corollary 6.8, (6.56), and (6.53) together, one has the global pointwise structure of $\mathrm{f}\left(x, t ; y_{0}\right)$. It yields the following lemma for the structure around a shock front.

Lemma 6.11. The solution $\mathrm{f}\left(x, t ; y_{0}\right)$ with $y_{0}<-\varepsilon^{-1}$ satisfies

$$
\chi_{+}(x)\left\|\mathrm{f}\left(x, t ; y_{0}\right)\right\|_{L_{\xi, 3}^{\infty}} \leq O(1) \frac{e^{-\frac{\left(y_{0}+\lambda_{3}-t\right)^{2}}{D_{0}(1+t)}}}{(1+t)} e^{-\frac{2 \varepsilon|x|}{D_{0}}} .
$$

One can rewrite (6.51) as $\left(\partial_{t}+\xi^{1} \partial_{x}-\mathrm{L}_{-}\right) \mathrm{f}\left(x, t ; y_{0}\right)=\left(\mathrm{L}_{\varphi}-\mathrm{L}_{-}\right) \mathrm{f}\left(x, t ; y_{0}\right)$ to yield that

$$
\begin{array}{r}
\mathrm{f}\left(x, t ; y_{0}\right)=\int_{\mathbb{R}} \mathbb{G}_{-}(x-y, t) \mathrm{f}\left(y, 0 ; y_{0}\right) d y+\int_{0}^{t} \int_{\mathbb{R}} \mathbb{G}_{-}(x-y, t-s)\left(\mathrm{L}_{\varphi}-\mathrm{L}_{-}\right) \\
\cdot\left[\mathrm{f}_{O}\left(y, s ; y_{0}\right)+\int_{0}^{s} \mathbb{G}_{\varphi}^{s-\tau}\left[\left(\mathfrak{C}^{t}+\mathfrak{C}^{c}+\mathfrak{C}^{m}\right)(\cdot, \tau)\right](y) d \tau\right] d y d s,
\end{array}
$$

so that the RHS of (6.60) can have exponentially sharp estimates. This representation (6.60) gives an operator defined on any data concentrated in the region $\left|x-y_{0}\right| \leq 1$, and we denote it by $\overline{\mathbb{G}}_{\varphi}$ :

$$
\overline{\mathbb{G}}_{\varphi}^{t}\left[\mathrm{f}\left(\cdot, 0 ; y_{0}\right)\right](x) \equiv \mathrm{f}\left(x, t ; y_{0}\right),
$$

where $\mathrm{f}\left(x, t ; y_{0}\right)$ is the solution of (6.51).

By Lemma 6.11 on the estimate $\mathrm{f}\left(x, t ; y_{0}\right)$ around the shock layer $\varphi$ and by the property that both $\mathrm{f}\left(x, 0 ; y_{0}\right)$ and $\left(\mathrm{L}_{\varphi}-\mathrm{L}_{-}\right) \mathrm{f}\left(y, s ; y_{0}\right)$ are purely microscopic, one has the following theorem from (6.60) and Corollary 3.1 with the bootstrap 
improvement:

Theorem 6.12. The solution of 6.51) with the conditions $y_{0}<-\varepsilon^{-1}$ and $\mathrm{P}_{0}^{-} \mathrm{f}\left(x, 0 ; y_{0}\right) \equiv 0$ satisfies

$$
\left\|\overline{\mathbb{G}}_{\varphi}^{t}\left[\mathrm{f}\left(\cdot, 0 ; y_{0}\right)\right](x)\right\|_{L_{\xi, 3}^{\infty}} \leq O(1) \sum_{j=1}^{3} \frac{e^{-\frac{\left(x-y_{0}-\lambda_{j}^{-} t\right)^{2}}{D_{1}(t+1)}}}{(t+1)}+e^{-\left(\left|x-y_{0}\right|+t\right) / D_{1}}
$$

$+O(1) \varepsilon \int_{0}^{t} \int_{\mathbb{R}}\left(\sum_{j=1}^{3} \frac{e^{-\frac{\left(x-y-\lambda_{j}^{-}(t-s)\right)^{2}}{D_{1}(t-s+1)}}}{(t-s+1)}+e^{-(|x-y|+t-s) / D_{1}}\right) \frac{e^{-\frac{\left(y_{0}+\lambda_{3}^{-} s\right)^{2}}{D_{1}(1+s)}}}{(1+s)} e^{-\frac{3 \varepsilon|y|}{D_{1}}} d y d s$.

Remark 6.13. One can remove the condition $\mathrm{P}_{0}^{-} \mathrm{f}\left(x, 0 ; y_{0}\right) \equiv 0$ by adding a term $O(1) \int_{0}^{t} \frac{e^{-\frac{\left(y_{0}-\varepsilon s\right)^{2}}{C_{0} s}}}{s} d s \varphi^{\prime}$ to the RHS of (6.62).

6.3. Summary on estimates of the linearized equation around the shock layer. In this subsection we summarize the linear estimates for the linearized equation

$$
\left\{\begin{array}{l}
\partial_{t} \mathrm{~h}+\xi^{1} \partial_{x} \mathrm{~h}-\mathrm{L}_{\varphi} \mathrm{h}=0 \\
\mathrm{~h}(x, 0)=\mathrm{h}_{0}(x)
\end{array}\right.
$$

with respect to various types of confined initial datum:

$$
\left\{\begin{array}{l}
\left\|\mathrm{h}_{0}\right\|_{L_{x}^{\infty}\left(L_{\xi, 3}^{\infty}\right)} \leq O(1) \\
\mathrm{h}_{0}(x) \equiv 0 \text { for }\left|x-y_{0}\right| \geq 1
\end{array}\right.
$$

Theorem 6.14. The solution of initial value problem (6.63) with the confined initial data $\mathrm{h}_{0}$ in (6.64) satisfies the following cases.

Case 1. Purely macroscopic transverse initial data around shock layer, i.e. $y_{0}=0$, $\mathrm{h}_{0}(x)^{c}=\mathrm{h}_{0}(x)^{m}=0$ for all $x \in \mathbb{R}$.

The solution of (6.63) satisfies

$$
\begin{array}{r}
\|\mathrm{h}(x, t)\|_{L_{\xi, 3}^{\infty}} \\
\leq O(1)\left[\chi_{-}(x)\left(\sum_{j=1}^{2} \frac{e^{-\frac{\left(x-\lambda_{j}^{-} t\right)^{2}}{C_{0}(t+1)}}}{\sqrt{(t+1)}}+\frac{e^{-\frac{(x-\varepsilon t)^{2}}{C_{0}(t+1)}}}{(t+1)}\right)\right] \\
\left.+\chi_{+}(x)\left(\sum_{j=1}^{2} \frac{e^{-\frac{\left(x-\lambda_{j}^{+} t\right)^{2}}{C_{0}(t+1)}}}{\sqrt{(t+1)}}+\frac{e^{-\frac{(x+\varepsilon t)^{2}}{C_{0}(t+1)}}}{(t+1)}\right)\right] \\
+O(1) \varepsilon^{2} \mathscr{A}_{2}(x, t)+O(1) \varepsilon^{19 / 20} e^{\frac{-3 \varepsilon|x|}{D_{1}}} \frac{e^{-|\varepsilon|^{2} t / E_{0}}}{(1+t)},
\end{array}
$$


where $\mathscr{A}_{2}$ is given in $(6.28)$ :

$$
\begin{aligned}
& \mathscr{A}_{2}(x, t) \\
& =\left\{\begin{array}{l}
\varepsilon^{-1} \frac{e^{-\frac{3 \varepsilon|x|}{D_{1}}-\frac{\varepsilon^{2} t}{E}}}{(t+1)} \text { for } x>0, \\
\sum_{j=1}^{2}\left(\frac{\sqrt{|x|+1}}{\left(x-\lambda_{j}^{-} t\right)(1+\varepsilon \sqrt{x+1})}+\frac{\varepsilon^{-1} e^{-C \varepsilon x}}{(t+1)}+\log \left(\varepsilon^{-1}\right) e^{-C \varepsilon\left|x-\lambda_{j}^{-} t\right|}\right) e^{-\frac{\varepsilon^{2}\left|x-\lambda_{j}^{-} t\right|}{\lambda_{j}^{-} E}} \\
\chi_{\left[\lambda_{j}^{-}(t+1)+\sqrt{t+1}, 0\right]}(x) \\
+\left(\log (t+1)\left(e^{-C \varepsilon t}+(1+\varepsilon \sqrt{t})^{-1}\right)+\frac{\varepsilon^{-1} e^{-C \varepsilon t}}{(t+1)}\right) \\
\chi_{\left[-\sqrt{t+1}+\lambda_{j}^{-}(t+1), \sqrt{t+1}+\lambda_{j}^{-}(t+1)\right]}(x) \\
+\left[\frac{\log (\sqrt{t+1}) e^{-\frac{\left(x-\lambda_{j}^{-} t\right)^{2}}{D_{1}(t+1)}}+\frac{(t+1)^{\frac{1}{2}} e^{-C \varepsilon\left|x-\lambda_{j}^{-} t\right|}}{\left(x-\lambda_{j}^{-} t\right)}}{(1+\varepsilon \sqrt{t})}\right.
\end{array}\right] \chi_{\left[-\infty, \lambda_{j}^{-}(t+1)-\sqrt{t+1]}(x)\right.}\left(\begin{array}{l}
\log \left(\varepsilon^{-1}\right) e^{-C \varepsilon\left|x-\lambda_{j}^{-} t\right|} e^{-C \varepsilon^{2} t}+e^{-\frac{3 \varepsilon\left|x-\lambda_{j}^{-} t\right|}{D_{1}}} \\
+\left[\frac{e^{-C \varepsilon\left(\lambda_{j}^{-} t-x\right)}}{\left(x-\lambda_{j}^{-} t\right)^{\frac{1}{2}}}+\frac{\varepsilon^{-1} e^{-C \varepsilon t} e^{-\frac{3 \varepsilon\left(\left|x-\lambda_{j}^{-} t\right|\right)}{D_{1}}}}{(t+1)}\right] \chi_{\left[-\infty, \lambda_{j}^{-}(t+1)-\sqrt{t+1]}\right.}(x) \text { for } x<0 .
\end{array}\right.
\end{aligned}
$$

Case 2. Purely compressive initial data around the shock layer, i.e. $y_{0}=0, \mathrm{~h}_{0}(x)^{t}=$ $\mathrm{h}_{0}(x)^{m}=0$ for all $x \in \mathbb{R}$.

$$
\left\{\begin{array}{l}
\left\|\mathrm{h}(x, t)-c^{*} \varphi^{\prime}(x)\right\|_{L_{\xi, 3}^{\infty}} \leq O(1) \frac{e^{-3 \frac{\varepsilon|x|}{D_{1}}-\frac{\varepsilon^{2} t}{E_{0}}}}{(1+t)^{\frac{1}{2}}}+O(1) \varepsilon \mathscr{A}_{2}(x, t), \\
c^{*}=\int_{\mathbb{R}}\left(\mathrm{I}_{3}, \mathrm{~h}_{0}\right) d x /\left(\mathrm{I}_{3},\left(\mathrm{M}_{+}-\mathrm{M}_{-}\right) / \sqrt{\mathrm{M}_{-}}\right) .
\end{array}\right.
$$

Case 3. Purely microscopic initial data around the shock layer, i.e. $y_{0}=0, \mathrm{~h}_{0}(x)^{t}=$ $\mathrm{h}_{0}(x)^{c}=0$ for all $x \in \mathbb{R}$.

$$
\|\mathrm{h}(x, t)\|_{L_{\xi, 3}^{\infty}} \leq O(1) \min \left(\frac{e^{-3 \frac{\varepsilon|x|}{D_{1}}-\frac{\varepsilon^{2} t}{E_{0}}}}{(1+t)}, \frac{\varepsilon \mathscr{A}_{2}(x, t)}{\sqrt{(1+t)}}\right)
$$

Case 4. Purely microscopic initial data outside the shock layer, i.e. $y_{0}<0, \mathrm{~h}_{0}(x)^{t}=$ $\mathrm{h}_{0}(x)^{c}=0$ for all $x \in \mathbb{R}$.

$$
\|\mathrm{h}(x, t)\|_{L_{\xi, 3}^{\infty} \leq O(1)} \sum_{j=1}^{3} \frac{e^{-\frac{\left(x-y_{0}-\lambda_{j}^{-} t\right)^{2}}{D_{1}(t+1)}}}{(t+1)}+e^{-\left(\left|x-y_{0}\right|+t\right) / D_{1}}
$$

$+O(1) \varepsilon \int_{0}^{t} \int_{\mathbb{R}}\left(\sum_{j=1}^{3} \frac{e^{-\frac{\left(x-y-\lambda_{j}^{-}(t-s)\right)^{2}}{D_{1}(t-s+1)}}}{(t-s+1)}+e^{-(|x-y|+t-s) / D_{1}}\right) \frac{e^{-\frac{\left(y_{0}+\lambda_{3}^{-} s\right)^{2}}{D_{1}(1+s)}}}{(1+s)} e^{-\frac{3 \varepsilon|y|}{D_{1}}} d y d s$.

Remark 6.15. The first three cases are used to compute the ansatz due to the initial data, and the last case is used for the nonlinear coupling outside the shock layer region. The second case is the only estimate containing a non-time decaying estimate around the shock layer. 
The reason why one only considers the purely microscopic component for the nonlinear coupling is that the nonlinear collision operator is purely microscopic. The reason why in the last case one considers $y<0$ is that in the region $y>0$, which is the supersonic region, all wave around the shock near the shock layer will be dissipated exponentially fast in time. One can ignore the nonlinear coupling in this region.

\section{Nonlinear stability of a Shock layer (Proof of Main Theorem)}

The perturbation $\mathrm{v}(x, t) \equiv\left(\mathrm{F}(x, t)-\varphi\left(x-x_{0}, t\right)\right) / \sqrt{\mathrm{M}_{-}}$of the Boltzmann shock profile $\varphi(x)$ satisfies

$$
\left\{\begin{array}{l}
\partial_{t} \mathrm{v}+\xi^{1} \partial_{x} \mathrm{v}=\mathrm{L}_{\varphi} \mathrm{v}+\mathscr{N}(\mathrm{v}), \\
\|\mathrm{v}(x, 0)\|_{L_{\xi, 3}^{\infty}} \leq O(1) \delta \varepsilon e^{-\frac{3 \varepsilon|x|}{D_{0}}}, \\
\int_{\mathbb{R}} \mathrm{v}^{c}(x, 0) d x=0,
\end{array}\right.
$$

where

$$
\mathscr{N}(\mathrm{v}) \equiv \frac{1}{\sqrt{\mathrm{M}_{-}}} Q\left(\sqrt{\mathrm{M}_{-} \mathrm{v}}\right)
$$

The condition (1.9) gives rise to the fact that the total mass of the perturbation $\int_{\mathbb{R}} \mathrm{v}(x, 0) d x$ is contained in the transverse components. Thus, the total mass of the perturbation does not contain any of the compressive components, i.e.

$$
\int_{\mathbb{R}} \mathrm{v}^{c}(x, 0) d x=0
$$

Thus, the third condition in (7.1) is a nature condition by choosing a suitable shock front $\varphi\left(x-x_{0}\right)$ to monitor the decay of the perturbations.

For simplicity, one may assume

$$
x_{0}=0 \text {. }
$$

The shock wave is a stationary 3 -shock wave and the initial data of the perturbation is exponentially decaying in $x$ so that one can expect that the solution remains exponentially decaying in $x>0$. Thus, one would expect that $\chi_{+}(x) \mathscr{N}(\mathrm{v})$ decays exponentially in $x$ as $|x| \rightarrow \infty$. Thus, the operator $\mathbb{G}_{\varphi}^{t}$ defined in (6.50) can be applied to the component $\chi_{+}(x) \mathscr{N}(\mathrm{v})$ and we rewrite (7.1) as follows:

$$
\partial_{t} \mathrm{v}+\xi^{1} \partial_{x} \mathrm{v}=\mathrm{L}_{\varphi} \mathrm{v}+\chi_{-} \mathscr{N}(\mathrm{v})+\chi_{+} \mathscr{N}
$$

The representation of $\mathrm{v}(x, t)$ is

$$
+\int_{0}^{t} \mathbb{G}_{\varphi}^{t-s}\left[\chi_{+}(\cdot) \mathscr{N}[\mathrm{v}](\cdot, s)\right] d s(x)+\sum_{j=-\infty}^{\infty} \int_{0}^{t} \overline{\mathbb{G}}_{\varphi}^{t-s}\left[\chi_{[2 j-1,2 j+1)}(\cdot) \chi_{-}(\cdot) \mathscr{N}[\mathrm{v}](\cdot, s)\right](x) d s
$$


where $\chi_{[2 j-1,2 j+1)}(x)$ is the characteristic function of the interval $[2 j-1,2 j+1)$, and $\overline{\mathbb{G}}_{\varphi}^{t}$ is the operator defined in (6.61). Denote

$$
\left\{\begin{array}{l}
J_{1} \equiv\left\|\int_{0}^{t} \mathbb{G}_{\varphi}^{t-s}\left[\chi_{+}(\cdot) \mathscr{N}[\mathrm{v}](\cdot, s)\right] d s(x)\right\|_{L_{\xi, 3}^{\infty}}, \\
J_{2} \equiv \sum_{j=-\infty}^{\infty}\left\|\int_{0}^{t} \overline{\mathbb{G}}_{\varphi}^{t-s}\left[\chi_{[2 j-1,2 j+1)}(\cdot) \chi_{-}(\cdot) \mathscr{N}[\mathbf{v}](\cdot, s)\right](x) d s\right\|_{L_{\xi, 3}^{\infty}} .
\end{array}\right.
$$

From that, $\mathscr{N}$ is purely microscopic. From (7.3) and Corollary 6.8 together there is a constant $\mathscr{C}_{0}>0$ such that

$$
\begin{aligned}
& \|\mathrm{v}(x, t)\|_{L_{\xi, 3}^{\infty} \leq J_{1}+J_{2}} \\
& +\mathscr{C}_{0} \delta\left\{\begin{array}{l}
\sum_{j=1}^{2} \varepsilon e^{-\frac{\varepsilon\left|x-\lambda_{j}^{-} t\right|}{D_{1}}}+\varepsilon e^{-\frac{\varepsilon|x|}{D_{1}}}+\varepsilon^{2} \mathscr{A}_{2}(x, t) \text { for } t \in\left(0, \varepsilon^{-2}\right), \\
\sum_{j=1}^{2}\left(\frac{e^{-\frac{\left(x-\lambda_{j}^{-} t\right)^{2}}{D_{1}(t+1)}}}{\sqrt{(t+1)}}+O(1) \varepsilon e^{-\frac{\varepsilon\left|x-\lambda_{j}^{-} t\right|}{4 D_{1}}} \chi_{\left(-\infty, \lambda_{j}^{-} t-\sqrt{1+t}\right)}(x)\right) \\
+\varepsilon^{2} \mathscr{A}_{2}(x, t)+\frac{e^{-\frac{\varepsilon|x|}{D_{1}}-\frac{\varepsilon^{2} t}{E_{0}}}}{\sqrt{t+1}} \text { for } t \geq \varepsilon^{-2} .
\end{array}\right.
\end{aligned}
$$

In order to continue to evaluate $J_{1}$ and $J_{2}$, we need to make an ansatz assumption on $v$ as follows.

\section{Ansatz Assumption.}

For $t \in\left(0, \varepsilon^{-2}\right)$,

$$
\begin{aligned}
& \|\mathrm{v}(x, t)\|_{L_{\xi, 3}^{\infty}} \leq O(1) \delta\left(\sum_{j=1}^{2} \varepsilon e^{-\frac{\left(x-\lambda_{j}^{-} t\right)^{2}}{D_{1}(t+1)}}\right. \\
& \left.\quad+\varepsilon e^{-\frac{\varepsilon\left|x-\lambda_{j}^{-} t\right|}{4 D_{1}}} \chi_{\left(-\infty, \lambda_{j}^{-} t-\sqrt{1+t]}\right.}(x)+O(1) \frac{e^{-\frac{\varepsilon|x|}{D_{1}}-\frac{\varepsilon^{2} t}{E_{0}}}}{\sqrt{t+1}}+\varepsilon^{2} \mathscr{A}_{2}(x, t)\right) .
\end{aligned}
$$

For $t \geq \varepsilon^{-2}$,

$$
\begin{aligned}
& \|\mathrm{v}(x, t)\|_{L_{\xi, 3}^{\infty} \leq} \leq(1) \delta\left(\frac{\chi_{\left[\lambda_{1}^{-}(t+1)+\sqrt{t+1}, \lambda_{2}^{-}(t+1)-\sqrt{t+1}\right]}(x)}{\sqrt{\left|x-\lambda_{1}^{-} t\right|\left|x-\lambda_{2}^{-} t\right|}}+\frac{\chi_{\left[\lambda_{2}^{-}(t+1)+\sqrt{t+1}, 0\right]}(x)}{\sqrt{\left|x-\lambda_{2}^{-} t\right|\left|x-\lambda_{3}^{-} t\right|}}\right) \\
& +\mathscr{C}_{0} \delta\left(\sum_{j=1}^{2} \frac{e^{-\frac{\left(x-\lambda_{j}^{-} t\right)^{2}}{D_{1}(t+1)}}}{\sqrt{t+1}}+O(1) \varepsilon e^{-\frac{\varepsilon\left|x-\lambda_{j}^{-} t\right|}{4 D_{1}}} \chi_{\left(-\infty, \lambda_{j}^{-} t-\sqrt{1+t}\right]}(x)\right. \\
& \left.+\varepsilon^{2} \mathscr{A}_{2}(x, t)+O(1) \frac{e^{-\frac{\varepsilon|x|}{D_{1}}-\frac{\varepsilon^{2} t}{E_{0}}}}{\sqrt{t+1}}\right) .
\end{aligned}
$$

To verify the ansatz assumptions (7.5) for $t \in\left(0, \varepsilon^{-2}\right)$ is straightforward, and its proof is omitted. 
NONLINEAR WAVE PROPAGATIONS OVER A BOLTZMANN SHOCK PROFILE 1107

Next we continue the estimates of $J_{1}$ and $J_{2}$ in (7.4) with the ansatz assumption (7.6). Under this assumption, one has for $t \in\left(\varepsilon^{-2}, \infty\right)$

$$
\begin{aligned}
& \left\|\chi_{+} \mathscr{N}[\mathbf{v}](x, t)\right\|_{L_{\xi, 3}^{\infty}} \\
& \quad \leq O(1) \delta^{2}\left(\frac{e^{-\frac{\varepsilon^{2} t}{E_{0}}}}{(1+t)}+\frac{1}{(1+t)(1+\varepsilon(1+t))}\right) e^{-\frac{3 \varepsilon|x|}{D_{1}}} \text { for } t \in\left(\varepsilon^{-2}, \infty\right) .
\end{aligned}
$$

With this, one can apply Corollary 6.8 with (7.7) to yield that for $x<0$

$$
\begin{aligned}
& J_{1}(x, t) \leq O(1) \mathscr{C}_{0}^{2} \frac{\delta^{2}}{\varepsilon} \sum_{j=1}^{2} \int_{0}^{t}\left(\frac{e^{-\frac{\left(x-\lambda_{j}(t-s)\right)^{2}}{D_{1}(t-s)}}}{(t-s+1)}+\varepsilon^{2} \frac{\mathscr{A}_{2}(x, t-s)}{\sqrt{|x|+1}}\right) \\
& \left(\frac{e^{-\frac{2 \varepsilon^{2} s}{E_{0}}}}{(s+1)}+\frac{1}{(1+s)(1+\varepsilon s)}\right) d s \leq O(1) \mathscr{C}_{0}^{2} \frac{\delta^{2}}{\varepsilon} \sum_{j=1}^{2}\left(|\log (1+t)| \frac{e^{-\frac{\left(x-\lambda_{j}^{-} t\right)^{2}}{2 D_{1}(t+1)}}}{t+1}\right. \\
& \left.+\varepsilon \chi_{\left[\lambda_{j}^{-} t+\sqrt{t}, 0\right]}(x)|\log \varepsilon|\left(\frac{1}{(1+t)}+\frac{1}{\left|x-\lambda_{j}^{-} t\right| \sqrt{1+t}}\right)\right) \\
& +O(1) \mathscr{C}_{0}^{2} \delta^{2}\left(\sum_{j=1}^{2} e^{-\varepsilon\left|x-\lambda_{j}^{-} t\right|}\left(1-\chi_{\left[\lambda_{j}^{-} t-\sqrt{t+1}, \lambda_{j}^{-} t+\sqrt{t+1}\right]}\right)+\frac{e^{-\frac{\varepsilon|x|}{D_{1}}-\frac{\varepsilon^{2} t}{E_{0}}}}{(1+t)}\right) .
\end{aligned}
$$

To estimate $J_{2}$, from (6.62) it follows that

$$
\begin{gathered}
J_{2}(x, t) \leq O(1) \sum_{j=-\infty}^{\varepsilon^{-1}} \int_{0}^{t} \sum_{l=1}^{3} \int_{j-1}^{j}\left(\frac{e^{-\frac{\left(x-z-\lambda_{l}^{-}(t-s)\right)^{2}}{D_{1}(t-s+1)}}}{(t-s+1)}\right. \\
\left.+e^{-\frac{|x-z|+(t-s)}{D_{1}}}\right)\|\mathrm{v}(z, s)\|_{L_{\xi}^{2}}^{2} d z d s \\
+O(1) \varepsilon \sum_{j=-\infty}^{\varepsilon^{-1}} \int_{0}^{t} \sum_{l=1}^{3} \int_{j-1}^{j} \int_{s}^{t} \int_{\mathbb{R}}\left(\frac{e^{-\frac{\left(x-y-\lambda_{l}^{-}(t-\tau)\right)^{2}}{D_{1}(t-\tau+1)}}}{(t-\tau+1)}+e^{-\frac{|x-y|+(t-\tau)}{D_{1}}}\right)\|\mathrm{v}(z, s)\|_{L_{\xi}^{2}}^{2}
\end{gathered}
$$

$$
\begin{gathered}
\cdot \frac{e^{-\frac{\left(z+\lambda_{3}^{-}(\tau-s)\right)^{2}}{D_{1}(\tau-s)}}}{(\tau-s)} e^{-\frac{2 \varepsilon|y|}{D_{1}}} d y d \tau d z d s \\
\leq O(1) \int_{0}^{t} \sum_{l=1}^{3} \int_{-\infty}^{-\varepsilon^{-1}}\left(\frac{e^{-\frac{\left(x-z-\lambda_{l}^{-}(t-s)\right)^{2}}{D_{1}(t-s+1)}}}{(t-s+1)}+e^{-\frac{|x-z|+(t-s)}{D_{1}}}\right)\|\mathrm{v}(z, s)\|_{L_{\xi}^{2}}^{2} d z d s \\
+O(1) \varepsilon \int_{0}^{t} \sum_{l=1}^{3} \int_{-\infty}^{\varepsilon^{-1}} \int_{s}^{t} \int_{\mathbb{R}}\left(\frac{e^{-\frac{\left(x-y-\lambda_{l}^{-}(t-\tau)\right)^{2}}{D_{1}(t-\tau+1)}}}{(t-\tau+1)}+e^{-\frac{|x-y|+(t-\tau)}{D_{1}}}\right) \\
\cdot\|\mathrm{v}(z, s)\|_{L_{\xi}^{2}}^{2} \frac{e^{-\frac{\left(z+\lambda_{3}^{-}(\tau-s)\right)^{2}}{D_{1}(\tau-s)}}}{(\tau-s)} e^{-\frac{2 \varepsilon|y|}{D_{1}}} d y d \tau d z d s
\end{gathered}
$$




$$
\begin{aligned}
=O(1) \int_{0}^{t} \sum_{l=1}^{3} \int_{-\infty}^{-\varepsilon^{-1}}\left(\frac{e^{-\frac{\left(x-z-\lambda_{l}^{-}(t-s)\right)^{2}}{D_{1}(t-s+1)}}}{(t-s+1)}+e^{-\frac{|x-z|+(t-s)}{D_{1}}}\right)\|\mathrm{v}(z, s)\|_{L_{\xi}^{2}}^{2} d z d s \\
+O(1) \varepsilon \sum_{l=1}^{3} \int_{0}^{t} \int_{\mathbb{R}}\left(\left(\frac{e^{-\frac{\left(x-y-\lambda_{l}^{-}(t-\tau)\right)^{2}}{D_{1}(t-\tau+1)}}}{(t-\tau+1)}+e^{-\frac{|x-y|+(t-\tau)}{D_{1}}}\right) e^{-\frac{2 \varepsilon|y|}{D_{1}}}\right. \\
\left.\cdot \int_{0}^{\tau} \int_{-\infty}^{\varepsilon^{-1}}\|\mathrm{v}(z, s)\|_{L_{\xi}^{2}}^{2} \frac{e^{-\frac{\left(z+\lambda_{3}^{-}(\tau-s)\right)^{2}}{D_{1}(\tau-s)}}}{(\tau-s)} d z d s\right) d y d \tau \equiv J_{2,1}+J_{2,2} .
\end{aligned}
$$

By applying the ansatz assumption (7.6) in (7.9) and by Lemma A.1 Lemma A.2 and Lemma A.3 together one has

$$
\begin{aligned}
& \left|J_{2,1}\right| \leq O(1)\left(\mathscr{C}_{0} \delta\right)^{2}\left(\sum_{j=1}^{2} \frac{e^{-\frac{\left(x-\lambda_{j}^{-} t\right)^{2}}{D_{1}(t+1)}}}{(t+1)^{\frac{1}{2}}}\right. \\
& \left.+\frac{\chi_{\left[\lambda_{1}^{-}(t+1)+\sqrt{t+1}, \lambda_{2}^{-}(t+1)-\sqrt{t+1}\right]}}{\sqrt{\left|x-\lambda_{1}^{-} t\right|\left|x-\lambda_{2}^{-} t\right|}}+\frac{\chi_{\left[\lambda_{2}^{-}(t+1)+\sqrt{t+1}, 0\right]}}{\sqrt{\left|x-\lambda_{2}^{-} t\right|\left|x-\lambda_{3}^{-} t\right|}}\right) \\
& +O(1)\left(\mathscr{C}_{0} \delta\right)^{2}\left(\varepsilon \frac{e^{-\frac{\varepsilon\left|x-\lambda_{1}^{-} t\right|}{4 D_{1}}}}{t+1} \chi_{\left(-\infty, \lambda_{1}^{-} t-\sqrt{1+t}\right]}(x)\right. \\
& \left.+|\log \varepsilon| \min \left(\frac{1}{\sqrt{t+1}}, \frac{1}{\varepsilon(1+t)}\right) e^{-\frac{\varepsilon|x|}{D_{1}}}\right) \\
& \leq O(1) \mathscr{C}_{0}^{2} \delta\left(\sum_{j=1}^{2} \delta \frac{e^{-\frac{\left(x-\lambda_{j}^{-} t\right)^{2}}{D_{1}(t+1)}}}{(t+1)^{\frac{1}{2}}}+\delta \frac{\chi_{\left[\lambda_{1}^{-}(t+1)+\sqrt{t+1}, \lambda_{2}^{-}(t+1)-\sqrt{t+1}\right]}}{\sqrt{\left|x-\lambda_{1}^{-} t\right|\left|x-\lambda_{2}^{-} t\right|}}\right. \\
& \left.+\frac{\delta|\log \varepsilon|}{\sqrt{\varepsilon}} \frac{\chi_{\left[\lambda_{2}^{-}(t+1)+\sqrt{t+1}, 0\right]}}{\sqrt{\left|x-\lambda_{2}^{-} t\right|\left|x-\lambda_{3}^{-} t\right|}}\right)+O(1)\left(\mathscr{C}_{0} \delta\right)^{2}\left(\varepsilon \frac{e^{-\frac{\varepsilon\left|x-\lambda_{1}^{-} t\right|}{4 D_{1}}}}{t+1} \chi_{\left(-\infty, \lambda_{1}^{-} t-\sqrt{1+t]}\right.}(x)\right. \\
& \left.+|\log \varepsilon| \min \left(\frac{1}{\sqrt{t+1}}, \frac{1}{\varepsilon(1+t)}\right) e^{-\frac{\varepsilon|x|}{D_{1}}} \chi_{[0, \infty)}\right)
\end{aligned}
$$

and

$$
\begin{aligned}
e^{-2 \frac{\varepsilon|y|}{D_{1}}} \int_{0}^{\tau} \int_{-\infty}^{\varepsilon^{-1}}\|\mathrm{v}(z, s)\|_{L_{\xi}^{2}}^{2} \frac{e^{-\frac{\left(z+\lambda_{3}(\tau-s)\right)^{2}}{D_{1}(\tau-s)}}}{(\tau-s)} d z d s \\
\quad \leq O(1)\left(\mathscr{C}_{0} \delta\right)^{2}|\log \varepsilon| e^{-\frac{\varepsilon|y|}{D_{1}}} \min \left(\frac{1}{\sqrt{1+\tau}}, \frac{1}{\varepsilon(1+\tau)}\right)
\end{aligned}
$$


NONLINEAR WAVE PROPAGATIONS OVER A BOLTZMANN SHOCK PROFILE 1109

From (7.11) and the definition of $J_{2,2}$ in (7.9), one has that by Lemma A.4 and Lemma A.6 for $x<0$

$$
\begin{gathered}
J_{2,2} \leq O(1)\left(\mathscr{C}_{0} \delta\right)^{2} \varepsilon|\log \varepsilon| \sum_{l=1}^{3} \int_{0}^{t} \int_{\mathbb{R}}\left(\frac{e^{-\frac{\left(x-y-\lambda_{l}^{-}(t-\tau)\right)^{2}}{D_{1}(t-\tau+1)}}}{(t-\tau+1)}+e^{-\frac{|x-y|+(t-\tau)}{D_{1}}}\right) \\
\min \left(\frac{1}{\sqrt{\tau+1}}, \frac{1}{\varepsilon(1+\tau)}\right) e^{-\frac{\varepsilon|y|}{D_{1}}} d y d \tau \\
\leq O(1)\left(\mathscr{C}_{0} \delta\right)^{2} \varepsilon|\log \varepsilon| \sum_{j=1}^{2} \min \left(\mathbb{K}^{1,2 ; 0}\left(x, t ; \lambda_{j}^{-}, D_{1}\right), \frac{1}{\varepsilon} \mathbb{K}^{2,2 ; 0}\left(x, t ; \lambda_{j}^{-}, D_{1}\right)\right) \\
+O(1)\left(\mathscr{C}_{0} \delta\right)^{2} \varepsilon|\log \varepsilon| \min \left(\mathbb{M}^{1,2 ; 0}\left(x, t ; \lambda_{3}^{-}, D_{1}\right), \frac{1}{\varepsilon} \mathbb{M}^{2,2 ; 0}\left(x, t ; \lambda_{3}^{-}, D_{1}\right)\right) \\
\left.\leq O(1) \frac{\left(\mathscr{C}_{0} \delta\right)^{2}|\log \varepsilon|}{(1+\varepsilon \sqrt{|x|+1})} \sum_{j=1}^{2} \min \left(\frac{1}{\sqrt{\left(\left|x-\lambda_{1}^{-} t\right|+1\right)}}\right) \frac{1}{\left(\left|x-\lambda_{1}^{-} t\right|+1\right)}\right) \\
\cdot \chi_{\left[\lambda_{1}^{-}(1+t)+\sqrt{t+1}, 0\right]}(x) \\
\left.+O(1)\left(\mathscr{C}_{0} \delta\right)^{2} \sqrt{\varepsilon} \frac{\sum_{j=1}^{2}\left(e^{-\varepsilon\left(\left|x-\lambda_{j}^{-} t\right|+t\right) / D_{1}} \chi_{\left[-\infty, \lambda_{j}^{-}(t+1)-\sqrt{t+1}\right]}+e^{-\frac{\left(x-\lambda_{j}^{-}(t+1)\right)^{2}}{D_{1}(t+1)}}\right.}{\sqrt{1+t}}\right) \\
+O(1)\left(\mathscr{C}_{0} \delta\right)^{2}|\log \varepsilon| \min \left(\varepsilon, \frac{1}{\sqrt{1+t}}\right) \frac{e^{-\frac{\varepsilon|x|}{D_{1}}}}{\sqrt{1+t}}
\end{gathered}
$$

The estimates (7.8), (7.10), (7.12), and the condition $\delta \ll \varepsilon^{1 / 2} /|\log \varepsilon|$ conclude that

$$
\begin{gathered}
\left|J_{1}+J_{2}\right| \leq O(1) \delta\left[\delta \left(\frac{\chi_{\left[\lambda_{1}^{-}(t+1)+\sqrt{t+1}, \lambda_{2}^{-}(t+1)-\sqrt{t+1]}\right.}(x)}{\sqrt{\left|x-\lambda_{1}^{-} t\right|\left|x-\lambda_{2}^{-} t\right|}}\right.\right. \\
\left.+\frac{|\log \varepsilon|}{\sqrt{\varepsilon}} \frac{\chi_{\left[\lambda_{2}^{-}(t+1)+\sqrt{t+1}, 0\right]}(x)}{\sqrt{\left|x-\lambda_{2}^{-} t\right|\left|x-\lambda_{3}^{-} t\right|}}\right) \\
+\mathscr{C}_{0} \delta\left(\sum_{j=1}^{2} \frac{e^{-\frac{\left(x-\lambda_{j}^{-} t\right)^{2}}{D_{1}(t+1)}}}{\sqrt{t+1}}+O(1) \frac{e^{-\frac{\varepsilon|x|}{D_{1}}}}{\sqrt{t+1}}+O(1) \varepsilon \frac{e^{-\frac{\varepsilon\left|x-\lambda_{1}^{-} t\right|}{4 D_{1}}}}{\sqrt{t+1}} \chi_{\left(-\infty, \lambda_{1}^{-} t-\sqrt{1+t]}\right.}(x)\right. \\
\left.\left.+\varepsilon^{2} \mathscr{A}_{2}(x, t)\right)\right] .
\end{gathered}
$$


Thus, from this and (7.4) together, the ansatz assumption (17.6) is true for $t \in$ $\left(\varepsilon^{-2}, \infty\right)$ when $\delta \ll \varepsilon^{1 / 2} /|\log \varepsilon|$ and $\varepsilon \ll 1$, and Theorem 1.1 is proved.

\section{Appendix A. Computational lemmas and estimates}

For the convenience of the reading we list the notation used in this section.

$$
\begin{aligned}
& \theta^{\alpha}(x, t ; \lambda, D) \equiv \frac{e^{-\frac{(x-\lambda t)^{2}}{D(1+t)}}}{(1+t)^{\frac{\alpha}{2}}}, \\
& \Gamma^{\alpha}(t) \equiv \int_{0}^{t}(s+1)^{-\alpha / 2} d s \\
& =O(1)\left\{\begin{array}{c}
1 \quad \text { for } \alpha>2, \\
\log (t+1) \text { for } \alpha=2, \\
(t+1)^{(2-\alpha) / 2} \text { for } \alpha<2 .
\end{array}\right.
\end{aligned}
$$

We use the following functions in [15] to study the nonlinear wave interactions

$$
\begin{aligned}
& H\left(x, t ; \mu_{1}, \mu_{2}, \mu_{3} ; C\right) \\
& \quad \equiv \int_{0}^{t} \int_{\mu_{1}(\sigma+1)+\sqrt{\sigma+1}}^{\mu_{2}(\sigma+1)-\sqrt{\sigma+1}} \frac{e^{-\frac{\left(x-y-\mu_{3}(t-\sigma+1)\right)^{2}}{C_{0}(t-\sigma+1)}}}{(t-\sigma+1)} \frac{1}{\left(y-\mu_{1} \sigma\right)\left(\mu_{2} \sigma-y\right)} d y d \sigma,
\end{aligned}
$$

where $\mu_{1}<\mu_{2}$, and

$$
\begin{aligned}
I^{\alpha, \beta, \gamma}\left(x, t ; t_{1}, t_{2} ; \lambda, \mu, D\right) \equiv \int_{t_{1}}^{t_{2}} \int_{-\infty}^{\infty}(t-s)^{(-\beta+\gamma) / 2}(t-s+1)^{-\gamma / 2} e^{-\frac{[y-x+\lambda(t-s)]^{2}}{D(t-s)}} & \cdot \theta^{\alpha}(y, s ; \mu, D) d y d s .
\end{aligned}
$$

Here the function $I^{\alpha, \beta, \gamma}\left(x, t ; t_{1}, t_{2} ; \lambda, \mu, D\right)$ was first introduced in 8 to study the nonlinear wave interaction.

Set

$$
\begin{aligned}
\Gamma^{\alpha}(t) \equiv \int_{0}^{t}(s+1)^{-\alpha / 2} d s \\
=O(1)\left\{\begin{array}{l}
1 \text { for } \alpha>2, \\
\log (t+1) \text { for } \alpha=2, \\
(t+1)^{(2-\alpha) / 2} \text { for } \alpha<2 .
\end{array}\right.
\end{aligned}
$$

Lemma A.1 (Liu [8]). Suppose that $\alpha>0, \beta \geq \gamma \geq 0, \beta-\gamma<3$. Then

$$
\begin{gathered}
I^{\alpha, \beta}(x, t ; 0, t ; \lambda, \lambda ; D)=O(1)\left[(t+1)^{(-\beta+1) / 2} \Gamma^{\alpha-1}(t+1)\right. \\
\left.+(t+1)^{(-\alpha+1) / 2} \Gamma^{\beta-1}(t+1)\right] \theta(x, t ; \lambda, D) .
\end{gathered}
$$

In particular,

$$
I^{\alpha, \beta}(x, t ; 0, t ; \lambda, \lambda ; D)=\left\{\begin{array}{l}
O(1) \theta(x, t ; \lambda, D), \quad \text { for } \quad \alpha \geq 3, \beta=1, \\
O(1) \theta^{3 / 2}(x, t ; \lambda, D), \quad \text { for } \quad \alpha \geq 2.5, \beta=2 .
\end{array}\right.
$$

Lemma A.2 (Liu [8]). Suppose that $\alpha \geq 1, \beta \geq \gamma \geq 0, \beta-\gamma<3$ and that $\lambda<\mu$. Then for any given constant $E>D$,

$$
\begin{aligned}
& I^{\alpha, \beta, \gamma}(x, t ; 0, t ; \lambda, \mu, D)=O(1)(t+1)^{(-\beta+1) / 2} \Gamma^{\alpha-1}(\sqrt{t+1}) \theta(x, t ; \lambda, D) \\
& \quad+O(1)(t+1)^{(-\alpha+1) / 2} \Gamma^{\beta-1}(\sqrt{t+1}) \theta(x, t ; \mu, D)
\end{aligned}
$$




$$
+\left\{\begin{array}{l}
0, \quad \text { for } \quad x<\lambda(t+1)+\sqrt{t+1}, \quad \text { or } \quad x>\mu(t+1)-\sqrt{t+1} \\
O(1)\left[(t+1)^{(-\beta+1) / 2} \Gamma^{\alpha-1}(x-\lambda t) \theta(x, t ; \lambda, E)+(\mu t-x)^{(-\beta+1) / 2}\right. \\
\left.\cdot(x-\lambda t)^{(-\alpha+1) / 2}+(t+1)^{(-\alpha+1) / 2} \Gamma^{\beta-1}(\mu t-x) \theta(x, t ; \mu, E)\right] \\
\text { for } \quad \lambda(t+1)+\sqrt{t+1}<x<\mu(t+1)-\sqrt{t+1} .
\end{array}\right.
$$

In particular

$$
I^{\alpha, \beta, \gamma}(x, t ; 0, t ; \lambda, \mu, D)=\left\{\begin{array}{l}
O(1) \psi^{1 / 2}(x, t ; \lambda), \quad \text { for } \quad \alpha=2, \beta=1 \\
O(1)\left[\psi^{3 / 2}(x, t ; \lambda)+\bar{\psi}^{3 / 2}(x, t ; \mu)\right], \quad \text { for } \quad \alpha=3, \beta=2 .
\end{array}\right.
$$

Lemma A.3 (Liu-Yu [15]). For $\left|x-\mu_{i} t\right|=O(1) \sqrt{1+t}$,

$$
H\left(x, t ; \mu_{1}, \mu_{2}, \mu_{3} ; C\right) \leq O(1) \frac{(\log (2+t))^{2}}{1+t}
$$

For $\left|x-\mu_{i} t\right| \geq O(1) \sqrt{1+t}$, one has the following. If $\mu_{3}=\mu_{2}$, then

$$
\begin{aligned}
& H\left(x, t ; \mu_{1}, \mu_{2}, \mu_{3}, C\right) \\
& =O(1)\left\{\begin{array}{l}
\frac{(\log (2+t))^{2}}{(1+t)} e^{-\frac{\left(x-\mu_{1} t\right)^{2}}{C_{0}(t+1)}} \text { for } x<\mu_{1} t-\sqrt{1+t}, \\
\frac{\log (2+t) t^{-1 / 4}}{\sqrt{\left(x-\mu_{1} t\right)\left(\mu_{2} t-x\right)}} \text { for } \mu_{1} t+\sqrt{1+t}<x<\mu_{2} t-\sqrt{1+t}, \\
\frac{(\log (2+t))^{2}}{(1+t)} e^{-\frac{\left(x-\mu_{2} t\right)^{2}}{C_{0}(t+1)}} \text { for } \mu_{2} t+\sqrt{1+t}<x .
\end{array}\right.
\end{aligned}
$$

If $\mu_{3}<\mu_{1}<\mu_{2}$, then

(A.4)

$$
\begin{array}{r}
H\left(x, t ; \mu_{1}, \mu_{2}, \mu_{3} ; C\right) \\
=O(1)\left\{\begin{array}{l}
\frac{(\log (2+t))^{2}}{(1+t)} e^{-\frac{\left(x-\mu_{3}\right)^{2}}{C_{0}(t+1)}} \text { for } x<\mu_{3} t-\sqrt{1+t}, \\
\frac{\log (2+t) t^{-1 / 4}}{\sqrt{\left(\mu_{1} t-x\right)\left(x-\mu_{3} t\right)}} \text { for } \mu_{3} t+\sqrt{t}<x<\mu_{1} t-\sqrt{t}, \\
\frac{\log (2+t)}{\sqrt{\left(x-\mu_{2} t\right)} t} \text { for } \mu_{1} t+\sqrt{t}<x<\mu_{2} t-\sqrt{t}, \\
\frac{(\log (2+t))^{2}}{(1+t)} e^{-\frac{\left(x-\mu_{2} t\right)^{2}}{C_{0}(t+1)}} \text { for } x>\mu_{2} t+\sqrt{1+t} .
\end{array}\right.
\end{array}
$$


If $\mu_{1}<\mu_{3}<\mu_{2}$, then

$$
\begin{aligned}
& H\left(x, t ; \mu_{1}, \mu_{2}, \mu_{3} ; C\right) \\
& =O(1)\left\{\begin{array}{l}
\frac{(\log (2+t))^{2} e^{-\frac{\left(x-\mu_{1} t\right)^{2}}{C_{0}(t+1)}}}{t} \text { for } x \leq \mu_{1} t-\sqrt{1+t}, \\
\frac{\log (2+t)(1+t)^{-1 / 4}}{\sqrt{\left(x-\mu_{1} t\right)\left(\mu_{3} t-x\right)}} \text { for } \mu_{1} t+\sqrt{t} \leq x \leq \mu_{3} t-\sqrt{t}, \\
\frac{\log (2+t)(1+t)^{-1 / 4}}{\sqrt{\left(x-\mu_{3} t\right)\left(\mu_{2} t-x\right)}} \text { for } \mu_{3} t+\sqrt{t} \leq x \leq \mu_{2} t-\sqrt{t}, \\
\frac{(\log (2+t))^{2} e^{-\frac{\left(x-\mu_{2} t\right)^{2}}{C_{0}(t+1)}}}{t} \text { for } x \geq \mu_{2} t+\sqrt{1+t} .
\end{array}\right.
\end{aligned}
$$

We introduce the following damping waves similar to those in 8 with an extra decaying factor $e^{-\delta s}$ with $\delta>4 \varepsilon$ :

$$
\begin{aligned}
& \mathbb{K}^{\alpha, \beta ; \delta}(x, t ; \lambda, D) \equiv \int_{0}^{t} \int_{\mathbb{R}} \frac{e^{-\frac{(x-y-\lambda(t-s))^{2}}{D(t-s+1)}} e^{-\frac{4 \varepsilon|y|}{D}}-\delta s}{(t-s+1)^{\frac{\beta}{2}}(s+1)^{\frac{\alpha}{2}}} d y d s \\
& \mathbb{L}^{\alpha, \beta ; \delta}(x, t ; \varepsilon, D) \equiv e^{-\frac{4 \varepsilon x}{D}} \int_{0}^{t} \int_{-\infty}^{0} \frac{e^{-\frac{(x-y-\varepsilon(t-s))^{2}}{D(t-s+1)}} e^{-\frac{4 \varepsilon y}{D}-\delta s}}{(t-s+1)^{\frac{\beta}{2}}(s+1)^{\frac{\alpha}{2}}} d y d s \text { for } x>0, \\
& \mathbb{M}^{\alpha, \beta ; \delta}(x, t ; \varepsilon, D) \equiv \int_{0}^{t} \int_{0}^{\infty} \frac{e^{-\frac{(x-y-\varepsilon(t-s))^{2}}{D(t-s+1)}} e^{-\frac{4 \varepsilon y}{D}-\delta s}}{(t-s+1)^{\frac{\beta}{2}}(s+1)^{\frac{\alpha}{2}}} d y d s \text { for } x>0 .
\end{aligned}
$$

With some minor modification in the proofs in $[8$ one has the following Liu lemmas: 
Lemma A.4 (Liu). Suppose $\lambda>0$ and $|\lambda|=O(1)$. Then for any $\delta>4 \varepsilon$,

$$
\begin{aligned}
& \mathbb{K}^{\alpha, \beta ; \delta}(x, t ; \lambda, D)=
\end{aligned}
$$

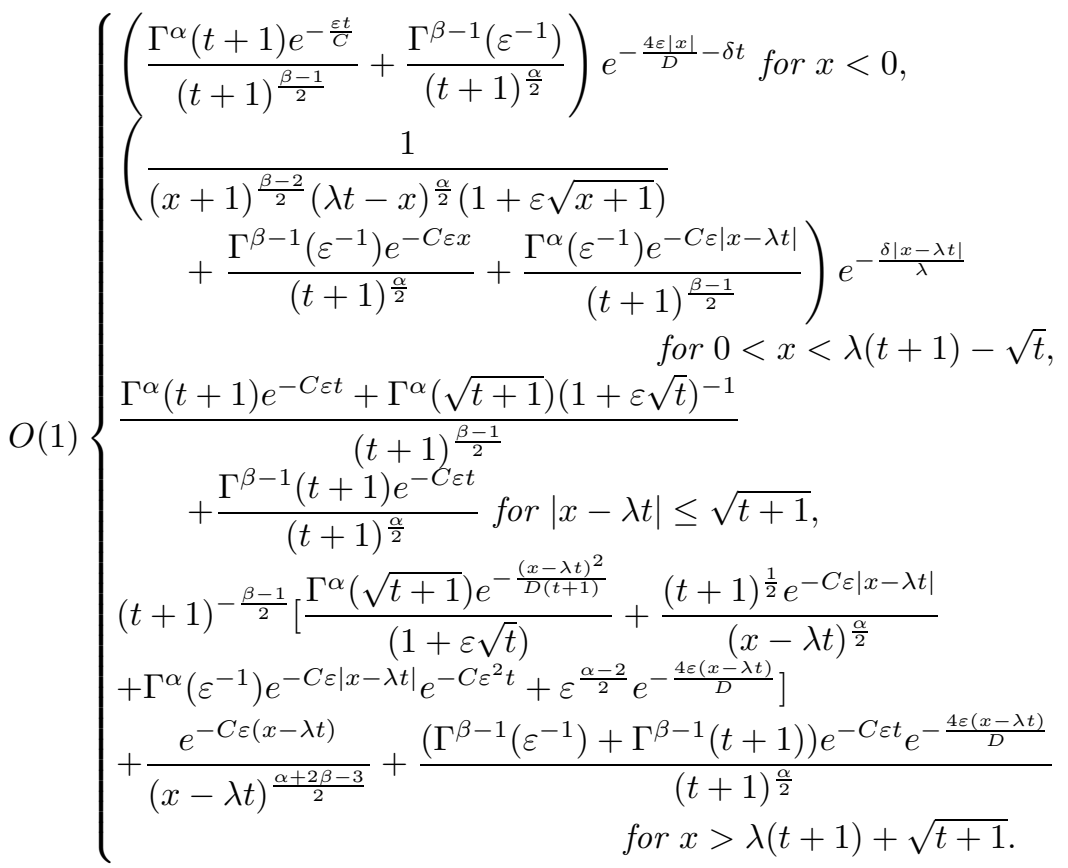

Lemma A.5 (Liu). Suppose that $\alpha \geq 0,3>\beta>0$, and $x \geq 0$. Then

$$
\begin{aligned}
& \mathbb{L}^{\alpha, \beta ; \delta}(x, t ; \varepsilon, D) \\
& \quad=O(1)\left[\frac{e^{-\frac{4 \varepsilon x}{D}-\frac{\varepsilon^{2} t}{4 D}-\frac{x^{2}}{D(t+1)}} \Gamma^{\alpha}(t+1)}{(t+1)^{(\beta-1) / 2}}+\frac{\varepsilon^{-3+\beta}\left(1-e^{-\frac{\varepsilon^{2} t}{2 D}}\right) e^{-2 \frac{x^{2}}{D(t+1)}}}{(t+1)^{\alpha / 2}} e^{-\delta t}\right] .
\end{aligned}
$$

In (A.7) the term $\varepsilon^{-3+\beta}$ can be replaced with $\Gamma^{\beta-1}(t+1)$.

Lemma A.6 (Liu). Suppose that $\alpha \geq 0,3>\beta \geq 0$, and $x \geq 0$. Then

$$
\mathbb{M}^{\alpha, \beta ; \delta}(x, t ; \varepsilon, D)=O(1)\left[\frac{\Gamma^{\alpha}(t+1) e^{-C \varepsilon(x+\varepsilon t)}}{(t+1)^{(\beta-1) / 2}}+\frac{\Gamma^{\beta-1}\left(\varepsilon^{-2}\right) e^{-C \varepsilon x}}{(t+1)^{\alpha / 2}} e^{-\delta t}\right]
$$

In (A.8) $\Gamma^{\beta-1}\left(\varepsilon^{-2}\right)$ can be replaced with $\Gamma^{\beta-1}(t+1)$.

Lemma A.7. Let $C_{0}, C_{1}, E_{0}, \omega_{0}$ be positive constants. Suppose that $\Lambda>0$ and $|\Lambda|=O(1)$. For $\beta \in[1,3], \alpha \in[1,2], \omega_{0} \in(0,1)$ we have the following cases. 
Case $\beta \in[1,2)$.

$$
\begin{aligned}
\int_{0}^{t} \int_{\mathbb{R}} \min (\varepsilon \sqrt{t-s+1}, 1) \theta^{\beta}\left(x-y, t-s, \Lambda, D_{0}\right) \frac{e^{-\frac{4 \varepsilon|y|}{D_{0}}-\frac{\varepsilon^{2} s}{E_{0}}}}{(1+s)^{\alpha / 2}} d y d s \\
\leq O(1)\left\{\begin{array}{l}
\varepsilon^{\frac{1-\omega_{0}}{2}} \mathbb{K}^{\alpha, \beta ; \frac{\varepsilon^{2}}{E_{0}}}\left(x, t ; \Lambda, D_{0}, E_{0}\right) \text { for }|x| \leq \varepsilon^{-1-\omega_{0}} \\
O(1) \mathbb{K}^{\alpha, \beta ; \frac{\varepsilon^{2}}{E_{0}}}\left(x, t ; \lambda, D_{0}, E_{0}\right) \text { for }|x| \geq \varepsilon^{-1-\omega_{0}}
\end{array}\right.
\end{aligned}
$$

Case $\beta \geq 2$.

$$
\begin{gathered}
\int_{0}^{t} \int_{\mathbb{R}} \min (\varepsilon \sqrt{t-s+1}, 1) \theta^{\beta}\left(x-y, t-s, \Lambda, D_{0}\right) \frac{e^{-\frac{4 \varepsilon|y|}{D_{0}}-\frac{\varepsilon^{2} s}{E_{0}}}}{(1+s)^{\alpha / 2}} d y d s \\
\leq O(1)\left\{\begin{array}{l}
\varepsilon \mathbb{K}^{\alpha, \beta-1 ; \frac{\varepsilon^{2}}{E_{0}}}\left(x, t ; \Lambda, D_{0}, E_{0}\right) \text { for }|x| \leq \varepsilon^{-1-\omega_{0}} \\
O(1) \mathbb{K}^{\alpha, \beta ; \frac{\varepsilon^{2}}{E_{0}}}\left(x, t ; \lambda, D_{0}, E_{0}\right) \text { for }|x| \geq \varepsilon^{-1-\omega_{0}}
\end{array}\right.
\end{gathered}
$$

Proof.

Case $|x| \geq \varepsilon^{-1-\omega_{0}}$.

For the case $\beta \in[1,2$ ), one just applies (A.6) to yield (A.9) and (A.10) for the case $|x| \geq \varepsilon^{-1-\omega_{0}}$.

Case $|x| \leq \varepsilon^{-1-\omega_{0}}$.

First, one considers the case $\beta \in[1,2)$. Break the integral as follows.

$$
\begin{aligned}
& \left(\int_{t-2 \varepsilon^{-\left(1+\omega_{0}\right)}}^{t}+\int_{0}^{t-2 \varepsilon^{-\left(1+\omega_{0}\right)}}\right) \\
& \cdot \int_{\mathbb{R}} \min (\varepsilon \sqrt{t-s+1}, 1) \theta^{\beta}\left(x-y, t-s, 1, D_{0}\right) \frac{e^{-\frac{4 \varepsilon|y|}{D_{0}}-\frac{\varepsilon^{2} s}{E_{0}}}}{(1+s)^{\alpha / 2}} d y d s \\
& \leq O(1)\left(\int_{t-2 \varepsilon^{-\left(1+\omega_{0}\right)}}^{t} \varepsilon^{\frac{1-\omega_{0}}{2}}+\int_{0}^{t-2 \varepsilon^{-\left(1+\omega_{0}\right)}}\right) \\
& \left\{\int_{\mathbb{R}} \theta^{\beta}\left(x-y, t-s, 1, D_{0}\right) \frac{e^{-\frac{4 \varepsilon|y|}{D_{0}}-\frac{\varepsilon^{2} t}{E_{0}}}}{(1+s)^{\alpha / 2}} d y\right\} d s \\
& \leq O(1)\left(\varepsilon^{\frac{1-\omega_{0}}{2}} \mathbb{K}^{\alpha, \beta ; \frac{\varepsilon^{2}}{E_{0}}}\left(x, t ; 1, D_{0}, E_{0}\right)\right. \\
& \left.+\int_{0}^{t-2 \varepsilon^{-\left(1+\omega_{0}\right)}} \frac{e^{-\frac{\varepsilon(t-s)}{4 D_{0}}-\frac{4 \varepsilon^{2} s}{E_{0}}}}{(t-s+1)^{(\beta-1) / 2}(1+s)^{\alpha / 2}} d s\right) \\
& \leq O(1) \varepsilon^{\frac{1-\omega_{0}}{2}} \mathbb{K}^{\alpha, \beta ; \frac{\varepsilon^{2}}{E_{0}}}\left(x, t ; 1, D_{0}, E_{0}\right)+O(1) \Gamma^{\beta-1}\left(\varepsilon^{-1}\right) e^{-\varepsilon_{0}^{-\frac{\omega_{0}}{8 D_{0}}}} \frac{e^{-\frac{\varepsilon^{2} t}{E_{0}}}}{(1+t)^{\alpha / 2}} \\
& \leq O(1) \varepsilon^{\frac{1-\omega_{0}}{2}} \mathbb{K}^{\alpha, \beta ; \frac{\varepsilon^{2}}{E_{0}}}\left(x, t ; 1, D_{0}, E_{0}\right) .
\end{aligned}
$$

This yields A.11 for the case $|x| \leq \varepsilon^{-1-\omega_{0}}$. 
For the situation $\beta \in[2,3)$, one still breaks the integral as follows.

$$
\begin{aligned}
& \left(\int_{t-2 \varepsilon^{-\left(1+\omega_{0}\right)}}^{t}+\int_{0}^{t-2 \varepsilon^{-\left(1+\omega_{0}\right)}}\right) \\
& \cdot \int_{\mathbb{R}} \min (\varepsilon \sqrt{t-s+1}, 1) \theta^{\beta}\left(x-y, t-s, 1, D_{0}\right) \frac{e^{-\frac{4 \varepsilon|y|}{D_{0}}-+\frac{\varepsilon^{2} s}{E_{0}}}}{(1+s)^{\alpha / 2}} d y d s \\
& \leq O(1)\left(\int_{t-2 \varepsilon^{-\left(1+\omega_{0}\right)}}^{t} \frac{\varepsilon}{\sqrt{(t-s+1)}}+\int_{0}^{t-2 \varepsilon^{-\left(1+\omega_{0}\right)}}\right) \\
& \left.\quad \cdot \int_{\mathbb{R}} \theta^{\beta}\left(x-y, t-s, 1, D_{0}\right) \frac{e^{-\frac{4 \varepsilon|y|}{D_{0}}-\frac{\varepsilon^{2} t}{E_{0}}}}{(1+s)^{\alpha / 2}} d y\right\} d s \\
& \leq O(1)\left(\varepsilon \mathbb{K}^{\alpha, \beta-1 ; \frac{\varepsilon^{2}}{E_{0}}}\left(x, t ; 1, D_{0}, E_{0}\right)\right. \\
& \left.+\int_{0}^{t-2 \varepsilon^{-\left(1+\omega_{0}\right)}} \frac{e^{-\frac{\varepsilon(t-s)}{4 D_{0}}-\frac{4 \varepsilon^{2} s}{E_{0}}}}{(t-s+1)^{(\beta-1) / 2}(1+s)^{\alpha / 2}} d s\right) \\
& \leq O(1) \varepsilon \mathbb{K}^{\alpha, \beta-1 ; \frac{\varepsilon^{2}}{E_{0}}}\left(x, t ; 1, D_{0}, E_{0}\right)+O(1) \Gamma^{\beta-1}\left(\varepsilon^{-1}\right) e^{-\varepsilon_{0}^{-\frac{\omega_{0}}{8 D_{0}}} \frac{e^{-\frac{\varepsilon^{2} t}{E_{0}}}}{(1+t)^{\alpha / 2}}} \\
& \leq O(1) \varepsilon \mathbb{K}^{\alpha, \beta-1 ; \frac{\varepsilon^{2}}{E_{0}}}\left(x, t ; 1, D_{0}, E_{0}\right) .
\end{aligned}
$$

Lemma A.8. For any $\alpha \geq 0$ and $\beta \geq 0$, with $E_{0} / 2>C_{0}>2 D_{0}$,

$$
\begin{aligned}
& \int_{0}^{t} \int_{\mathbb{R}} \frac{e^{-\frac{(x-y-\varepsilon(t-s))^{2}}{C_{0}(t-s)}}}{(1+t-s)^{\frac{\beta}{2}}} \frac{e^{-\frac{\varepsilon|y|}{D_{0}}-\frac{|\varepsilon|^{2} s}{E_{0}}}}{(1+s)^{\alpha / 2}} d y d s \\
& \leq O(1) e^{\frac{2 \varepsilon x}{C_{0}}-\frac{\varepsilon^{2} t}{E_{0}}}\left(\Gamma^{\beta-1}\left(\varepsilon^{-2}\right)(1+t)^{-\frac{\alpha}{2}}+\Gamma^{\alpha}\left(\varepsilon^{-2}\right) \frac{e^{-\left(\frac{1}{2 C_{0}}-\frac{1}{2 E_{0}}\right) \varepsilon^{2} t}}{(1+t)^{\frac{\beta-1}{2}}}\right) \text { for } x<0,
\end{aligned}
$$

and

$$
\begin{aligned}
& e^{-\frac{2 \varepsilon x}{C_{0}}} \int_{0}^{t} \int_{\mathbb{R}} \frac{e^{-\frac{(x-y-\varepsilon(t-s))^{2}}{C_{0}(t-s)}}}{(1+t-s)^{\frac{\beta}{2}}} \frac{e^{-\frac{\varepsilon|y|}{D_{0}}-\frac{|\varepsilon|^{2} s}{E_{0}}}}{(1+s)^{\alpha / 2}} d y d s \\
& \leq O(1) e^{-\frac{\varepsilon^{2} t}{E_{0}}}\left(\Gamma^{\beta-1}\left(\varepsilon^{-2}\right)(1+t)^{-\frac{\alpha}{2}}+\Gamma^{\alpha}\left(\varepsilon^{-2}\right) \frac{e^{-\left(\frac{1}{2 C_{0}}-\frac{1}{2 E_{0}}\right) \varepsilon^{2} t}}{(1+t)^{\frac{\beta-1}{2}}}\right) \text { for } x>0,
\end{aligned}
$$


(A.15)

$$
\begin{aligned}
& \int_{0}^{t} \int_{\mathbb{R}} \frac{e^{-\frac{(x-y-\varepsilon(t-s))^{2}}{C_{0}(t-s)}}}{(1+t-s)^{\frac{\beta}{2}}} \frac{e^{-\frac{\varepsilon|y|}{D_{0}}-\frac{|\varepsilon| s}{E_{0}}}}{(1+s)^{\alpha / 2}} d y d s \\
& \leq O(1) e^{\frac{2 \varepsilon x}{C_{0}}}\left(\frac{\Gamma^{\beta-1}\left(\varepsilon^{-2}\right) e^{-\frac{\varepsilon t}{E_{0}}}}{(1+t)^{\frac{\alpha}{2}}}+\frac{\Gamma^{\alpha}\left(\varepsilon^{-1}\right) e^{-\frac{\varepsilon^{2} t}{2 E_{0}}}}{(1+t)^{\frac{\beta-1}{2}}}\right) \text { for } x<0,
\end{aligned}
$$

and

$$
\begin{aligned}
& e^{-\frac{2 \varepsilon x}{C_{0}}} \int_{0}^{t} \int_{\mathbb{R}} \frac{e^{-\frac{(x-y-\varepsilon(t-s))^{2}}{C_{0}(t-s)}}}{(1+t-s)^{\frac{\beta}{2}}} \frac{e^{-\frac{\varepsilon|y|}{D_{0}}-\frac{|\varepsilon| s}{E_{0}}}}{(1+s)^{\alpha / 2}} d y d s \\
& \quad \leq O(1)\left(\frac{\Gamma^{\beta-1}\left(\varepsilon^{-2}\right) e^{-\frac{\varepsilon t}{E_{0}}}}{(1+t)^{\frac{\alpha}{2}}}+\frac{\Gamma^{\alpha}\left(\varepsilon^{-1}\right) e^{-\frac{\varepsilon^{2} t}{2 E_{0}}}}{(1+t)^{\frac{\beta-1}{2}}}\right) \text { for } x>0 .
\end{aligned}
$$

Proof.

$$
\begin{aligned}
& \int_{0}^{t} \int_{\mathbb{R}} \frac{e^{-\frac{(x-y-\varepsilon(t-s))^{2}}{C_{0}(t-s)}}}{(1+t-s)^{\frac{\beta}{2}}} \frac{e^{-\frac{\varepsilon|y|}{D_{0}}-\frac{\varepsilon^{2} s}{E_{0}}}}{(1+s)^{\alpha / 2}} d y d s \\
& \quad \leq \int_{0}^{t} \int_{\mathbb{R}} \frac{e^{-\frac{(x-y)^{2}}{C_{0}(t-s)}}}{(1+t-s)^{\frac{\beta}{2}}} \frac{e^{\frac{2 \varepsilon(x-y)}{C_{0}}-\frac{\varepsilon|y|}{D_{0}}-\frac{\varepsilon^{2}(t-s)}{C_{0}}-\frac{\varepsilon^{2} s}{E_{0}}}}{(1+s)^{\alpha / 2}} d y d s \\
& \quad \leq O(1) e^{\frac{2 \varepsilon x}{C_{0}}} \int_{0}^{t} \frac{e^{-\varepsilon^{2}\left(\frac{(t-s)}{C_{0}}+\frac{s}{E_{0}}\right)}}{(1+t-s)^{\frac{\beta-1}{2}}(1+s)^{\alpha / 2}} d s \\
& \quad \leq O(1) e^{\frac{2 \varepsilon x}{C_{0}}-\frac{\varepsilon^{2} t}{E_{0}}}\left(\Gamma^{\beta-1}\left(\varepsilon^{-2}\right)(1+t)^{-\frac{\alpha}{2}}+\Gamma^{\alpha}\left(\varepsilon^{-2}\right) \frac{e^{-\left(\frac{1}{2 C_{0}}-\frac{1}{2 E_{0}}\right) \varepsilon^{2} t}}{(1+t)^{\frac{\beta-1}{2}}}\right) .
\end{aligned}
$$

Similar calculations yield (A.15) and (A.16). This concludes the proof of this lemma.

Lemma A.9. Suppose that $\Lambda<0, C_{0}, D_{0}, E_{0}>0, D_{0}>4|\Lambda|$, and $|\Lambda|, C_{0}, D_{0}, E_{0}$ $=O(1)$. Then

$$
\begin{aligned}
\int_{0}^{t} \int_{\mathbb{R}} \theta^{\beta}\left(x-y, t-s ; \Lambda, C_{0}\right) e^{-\varepsilon(t-s)} \frac{e^{-\frac{\varepsilon|x|}{D_{0}}-\frac{\varepsilon^{2} s}{E_{0}}}}{(1+s)^{\alpha / 2}} d y d s \\
\quad \leq O(1) e^{-\frac{\varepsilon|x|}{D_{0}}}\left(\Gamma^{\beta-1}\left(\varepsilon^{-1}\right) \frac{e^{-\frac{\varepsilon^{2} t}{E_{0}}}}{(1+t)^{\alpha / 2}}+\Gamma^{\alpha}\left(\varepsilon^{-2}\right) \frac{e^{-\varepsilon t / 4}}{(1+t)^{(\beta-1) / 2}}\right) .
\end{aligned}
$$


Proof. We just verify the lemma for $x<0$ :

$$
\begin{aligned}
& \int_{0}^{t} \int_{\mathbb{R}} \theta^{\beta}\left(x-y, t-s ; \Lambda, C_{0}\right) e^{-\varepsilon(t-s)} \frac{e^{-\frac{\varepsilon|y|}{D_{0}}-\frac{\varepsilon^{2} s}{E_{0}}}}{(1+s)^{\alpha / 2}} d y d s \\
& \leq O(1) \int_{0}^{t} \int_{\mathbb{R}} e^{\frac{\varepsilon x}{D_{0}}} \theta^{\beta}\left(x-y, t-s, \Lambda-\frac{\varepsilon C_{0}}{2 D_{0}}, C_{0}\right) e^{-\frac{\varepsilon(t-s)}{4}-\frac{\varepsilon^{2} s}{E_{0}}} \frac{e^{-\frac{\varepsilon^{2} s}{E_{0}}}}{(1+s)^{\alpha / 2}} d y d s \\
& \leq O(1) \int_{0}^{t} \frac{e^{\frac{\varepsilon x}{D_{0}}-\frac{\varepsilon(t-s)}{2}-\frac{\varepsilon^{2} s}{E_{0}}}}{(t-s+1)^{\frac{\beta-1}{2}}(1+s)^{\alpha / 2}} \\
& \leq O(1) e^{-\frac{\varepsilon|x|}{D_{0}}}\left(\Gamma^{\beta-1}\left(\varepsilon^{-1}\right) \frac{e^{-\frac{\varepsilon^{2} t}{E_{0}}}}{(1+t)^{\alpha / 2}}+\Gamma^{\alpha}\left(\varepsilon^{-2}\right) \frac{e^{-\varepsilon t / 4}}{(1+t)^{(\beta-1) / 2}}\right) .
\end{aligned}
$$

\section{REFERENCES}

1. Caflisch, R. E.; Nicolaenko, B., Shock profile solutions of the Boltzmann equation, Comm. Math. Phys. 89 (1982), 161-194. MR676183 (84d:82022)

2. Goodman, J. Nonlinear asymptotic stability of viscous shock profiles for conservation laws, Arch. Rational Mech. Anal. 95 (1986), no. 4, 325-344. MR853782 (88b:35127)

3. Goodman, J. Stability of viscous scalar shock fronts in several dimensions. Trans. Amer. Math. Soc. 311 (1989), no. 2, 683-695 MR978372 (89j:35059)

4. Grad, H. Asymptotic theory of the Boltzmann equation, Physics of Fluids, No. 2, Vol. 6, (1963), 147-181. MR0155541 (27:5475)

5. Grad, H. Asymptotic equivalence of the Navier-Stokes and nonlinear Boltzmann equations, Proc. Symp. Appl. Math. 17 (ed., R. Finn), 154-183, AMS, Providence, 1965. MR0184507 $(32: 1979)$

6. Grad, H. Proc. Symp. in Appl. Math. 17, 154-183 (1965), Rarified Gas Dynamics, I, 26-59 (1963). MR0184507 (32:1979)

7. Liu, T.-P. Nonlinear stability of shock waves for viscous conservation laws, Mem. Amer. Math. Soc. 56 (1985), no. 328. MR791863 (87a:35127)

8. Liu, T.-P. Pointwise convergence to shock waves for viscous conservation laws, Comm. Pure Appl. Math. 50 (1997), no. 11, 1113-1182. MR1470318 (98j:35121)

9. Liu, T.-P.; Zeng, Y. Large time behavior of solutions for general quasilinear hyperbolicparabolic systems of conservation laws, Mem. Amer. Math. Soc., 125 (1997), no. 599. MR1357824 (97g:35107)

10. Liu, T.-P.; Zeng, Y. Time-asymptotic behavior of wave propagation around a viscous shock profile. Comm. Math. Phys. 290 (2009), no. 1, 23-82. MR 2520507

11. Liu, T.-P.; Yu, S.-H. Propagation of a stationary shock layer in the presence of a boundary. Arch. Rational Mech. Anal. 139 (1997), no. 1, 57-82 MR1475778(99b:35137)

12. Liu, T.-P.; Yu, S.-H. Boltzmann Equation: Micro-Macro Decompositions and Positivity of Shock Profiles, Comm. Math. Phys. 246 (2004), 133-179. MR2044894(2005f:82101)

13. Liu, T.-P.; Yu, S.-H. The Green's function and large-time behavior of solutions for onedimensional Boltzmann equation, Comm. Pure. Appl. Math. 57 (2004), no. 12, 1543-1608. MR2082240 (2005m:82132)

14. Liu, T.-P.; Yu, S.-H. Green's function of Boltzmann equation, 3-D waves, Bull. Inst. Math. Acad. Sin. (N.S.) 1 (2006), no. 1, 1-78. MR2230121 (2007k:82116)

15. Liu, T.-P.; Yu, S.-H., Initial-boundary value problem for one-dimensional wave solutions of the Boltzmann equation, Comm. Pure Appl. Math. 60 (2007), no. 3, 295-356. MR.2284213 (2009b:35025)

16. Liu, T.-P.; Yu, S.-H. Viscous shock wave tracing, local conservation laws, and pointwise estimates. Bull. Inst. Math. Acad. Sin. (N.S.) 4 (2009), no. 3, 235-297. MR 2571697

17. Liu, T.-P.; Yu, S.-H. Invariant manifolds for steady Boltzmann flows and its applications, preprint. 
18. Matsumura, A.; Nishihara, K. On the stability of travelling wave solutions of a onedimensional model system for compressible viscous gas. Japan J. Appl. Math. 2 (1985), no. 1, 17-25. MR.839317 (87j:35335a)

19. Sone, Y. Kinetic theory of evaporation and condensation linear and nonlinear problems, J. Phys. Soc. Jpn. 45 (1978), 315-320.

20. Sone, Y.; Aoki, K; Yamashita, K. A study of unsteady strong condensation on a plane condensed phase with special interest in formation of steady profile, in: V. Bofli and C. Cercignani, eds., Rarefied Gas Dynamics (Teubner, Stuttgart), Vol. II (1986), 323-333.

21. Sone, Y.; Golse, F.; Ohwada, T.; Doi, T. Analytical study of transonic flows of a gas condensing onto its plane condensed phase on the basis of kinetic theory, Eur. J. Mech. B/Fluids 17 (1998), 277-306. MR1632303 (99f:76116)

22. Sone, Y. Molecular Gas Dynamics: Theory, Techniques, and Applications, Birkhauser, 2007. MR:2274674 (2007m:82081)

23. Zeng, Y. $L^{1}$ asymptotic behavior of compressible, isentropic, viscous 1-D flow, Comm. Pure Appl. Math. 47 (1994), no. 8, 105-1082. MR.1288632 (95f:35209)

Department of Mathematics, National University of Singapore, 10 Lower Kent Ridge RoAd, Singapore, 119076

E-mail address: matysh@nus.edu.sg 